Portland State University

PDXScholar

Fall 12-4-2019

\title{
Passive Separation of Binary Fluid Mixtures in Microchannels Using Lattice Boltzmann Method
}

Mohanad Kamil Radhi

Portland State University

Follow this and additional works at: https://pdxscholar.library.pdx.edu/open_access_etds

Part of the Mechanical Engineering Commons

Let us know how access to this document benefits you.

\section{Recommended Citation}

Radhi, Mohanad Kamil, "Passive Separation of Binary Fluid Mixtures in Microchannels Using Lattice Boltzmann Method" (2019). Dissertations and Theses. Paper 5332.

https://doi.org/10.15760/etd.7205

This Dissertation is brought to you for free and open access. It has been accepted for inclusion in Dissertations and Theses by an authorized administrator of PDXScholar. Please contact us if we can make this document more accessible: pdxscholar@pdx.edu. 
Passive Separation of Binary Fluid Mixtures in Microchannels Using Lattice Boltzmann Method

by

Mohanad Kamil Radhi

A dissertation submitted in partial fulfillment of the requirements for the degree of

Doctor of Philosophy

in

Mechanical Engineering

Dissertation Committee:

Derek Tretheway, Chair

Mark Weislogel

Gerald Recktenwald

Dacian Daescu

Portland State University

2019 


\begin{abstract}
Fluid mixtures in real life exist in two forms: miscible and immiscible. Separation of these mixtures using chemical agents or thermal energy has both environmental and economic disadvantages. The high cost and the environmental damage caused by the traditional separation techniques have stimulated both academia and industry to seek alternatives. The emergence of microfluidics offers robust solutions for a broad span of transport problems due to the high surface to volume ratio and reduced length scales. Particularly, the separation efficiency increases significantly due to the aforementioned feature. However, there is still a pressing need of passive separations for the sake of energy minimization and environmental safety. This work focuses on passive separation of both miscible and immiscible mixtures through the surface forces in microchannels. In the miscible case two fluids of physical properties imitating those of water and ethanol are investigated using the multi-range multi-components Shan-Chen Lattice Boltzmann Method (LBM) for a 2D channel. The variations of the fluid-fluid and fluid-solid interaction coefficients of both fluids, the relaxation times, and the spacing between the walls are examined under static conditions. The uneven interactions are considered with static as well as flow conditions. Because the surface forces are of intermolecular nature, their spacial range is short and did not exceed $30 \mathrm{~nm}$ in our study. Therefore, we added solid posts distributed in patterns. The addition of these features enhanced the density jumps significantly between the upper and lower halves of the channel. In the immiscible case we studied how uneven
\end{abstract}


wetting conditions influence two-phase flow in a 3D T-shaped microchannel. The D3Q27 LBM model with Shan-Chen forcing was used to control the contact angles of the lower and upper halves of the channel separately. The feasibility of separation was examined by constructing the breakup and non-breakup regimes for capillary numbers $(\mathrm{Ca})$ ranging from 0.002 to 0.3 and droplet lengths $\left(L_{0}\right)$ ranging from 1.5 to 4 times the width of the channel $(W=30 \mu m)$. The difference between the upper and lower contact angles has the strongest impact on the breakup and non-breakup regimes. The geometrical parameters represented by the main channel aspect ratio $(\mathrm{AR})$ and side to main channel width ratio (WR) are also significant players as they shift the border of the breakup area significantly. 


\section{Acknowledgements}

I am extremely grateful to my adviser Dr. Tretheway for his valuable guidance, unparalleled support, and for introducing me to Lattice Boltzmann Method and passive separation. I would like to extend my sincere thanks to Professor Weislogel for his fruitful discussions and practical suggestions that made me indebted to him. I also wish to thank Dr. Recktenwald for his invaluable mentoring about parallel processing programming using the message passing interface. Special thanks to Professor Daescu for serving in my dissertation committee and for inspiring me to expand my knowledge in applied mathematics.

My great thanks to the Higher Committee of Education Development in Iraq (HCEDIRAQ) for their support during this long trip.

I am deeply indebted to my parents for their priceless care and love during my entire life.

Words are not enough to describe my acknowledgement to my beloved family starting from my amazing wife Makarim for providing me with encouragement and patience throughout the duration of the Ph.D study, my kids: Sarah, Anas, and Sama for filling my life with pleasure and happiness. 


\section{Table of Contents}

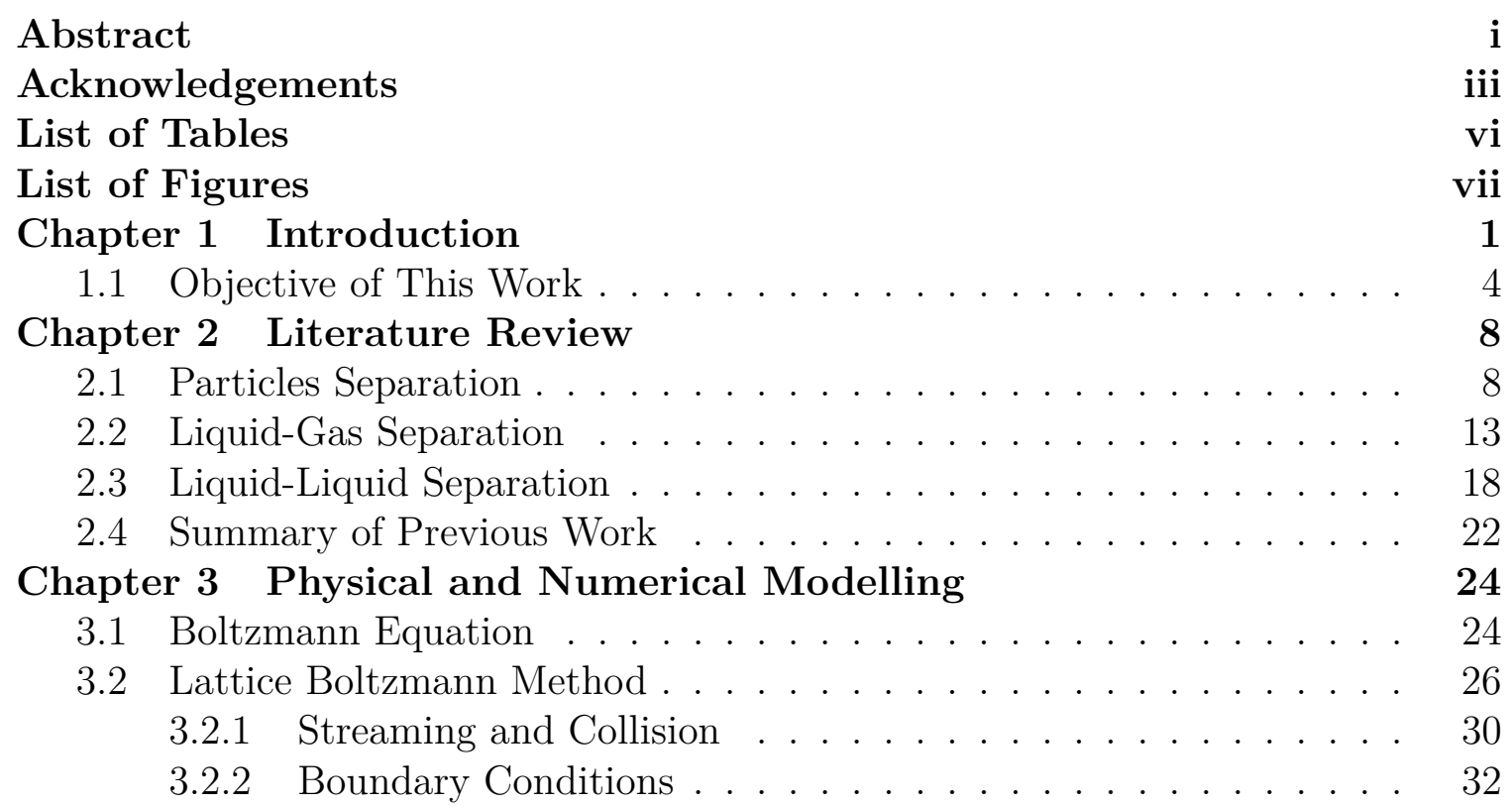

3.3 Shan Chen Model for Multiphase Multicomponent Flows . . . . . . . 36

3.3.1 Multirange Pseudopotential for Binary Mixture . . . . . . . 38

3.4 Physical Mapping . . . . . . . . . . . . . . . . . . . . . . . . 41

3.5 Contact Angle Measurements . . . . . . . . . . . . . . . 43

3.6 Young-Laplace Test . . . . . . . . . . . . . . . . . . . . . . . . . . . . . . . 46

3.7 Numerical Validation . . . . . . . . . . . . . . . . . . . . . . . 48

3.7.1 Two dimensional Poiseuille Flow . . . . . . . . . . . . . . 48

3.7 .2 Drop Breakup in a T-Junction . . . . . . . . . . . . . 51

Chapter 4 Miscible Binary Mixture in a Straight Channel $\quad 54$

4.1 Cross Interaction Effect . . . . . . . . . . . . . . . . 56

4.2 Solid-Fluid Interaction Coefficients Effect . . . . . . . . . . . . 60

4.3 Relaxation Times Effect . . . . . . . . . . . . . . . . 66

4.4 Height of channel effect . . . . . . . . . . . . . . . . . . 71

4.5 Uneven Wetting with Variable Heights . . . . . . . . . . . 75

4.6 Solid Posts Enhancement . . . . . . . . . . . . . . . . . . . . . . 79

$4.6 .1 \quad$ Static Case . . . . . . . . . . . . . . . . . . . . 79

4.6 .2 Dynamic Case . . . . . . . . . . . . . . . . . . . . 85 
$\begin{array}{lll}\text { Chapter } 5 & \text { Two-Phase Flow Separation } & 92\end{array}$

5.1 Initialization Process . . . . . . . . . . . . . . . . . . . . 93

5.2 Uneven Wettability Dynamic Effect . . . . . . . . . . . . . . . 102

5.3 Initial Length and Capillary Number Effect . . . . . . . . . . . . . 112

5.4 Regimes of Results . . . . . . . . . . . . . . . . . . . 115

5.4.1 Effect of Wetting Conditions . . . . . . . . . . . . . . 115

5.4 .2 Effect of Viscosity Ratio . . . . . . . . . . . . . . . . . 119

5.4 .3 Effect of Aspect Ratio . . . . . . . . . . . . . . . . . . 122

5.4.4 Effect of Side to Main Channel Width Ratio . . . . . . . . . 125

$\begin{array}{lll}\text { Chapter } 6 & \text { Conclusions and Recommendations } & 127\end{array}$

6.1 Conclusions . . . . . . . . . . . . . . . . . . . . . . . . 128

6.1.1 Miscible Mixture Conclusions . . . . . . . . . . . . . . . 128

6.1.2 Two-Phase Flow Conclusions . . . . . . . . . . . . . . . 129

6.2 Future Work . . . . . . . . . . . . . . . . . . . . . . 130

Bibliography $\quad 133$

$\begin{array}{lll}\text { Appendix A Computer Programs } & 141\end{array}$

A.1 Computational Expense . . . . . . . . . . . . . . . . . . . . . 141

A.2 Miscible Case Flowchart . . . . . . . . . . . . . . . . . . 142

A.3 Miscible Case Code . . . . . . . . . . . . . . . . . . . . 142

A.4 Immiscible Case Flowchart . . . . . . . . . . . . . . . . . . . 161

A.5 Immiscible Case Code . . . . . . . . . . . . . . . . . . . . . . 162 


\section{List of Tables}

1.1 Scaling laws for different physical quantities $\ldots \ldots \ldots \ldots$

3.1 The extended force weights up to the 6 th belt in $2 \mathrm{D} \ldots \ldots \ldots$

3.2 Resulting Contact Angles due to Solid-Fluid interaction parameters variations. . . . . . . . . . . . . . . . . . 45

3.3 Numerical results of Young-Laplace test for spherical drops of fluid-1 surrounded by fluid-2 at $G_{12}=6.0, G_{11}=-0.75 \ldots \ldots \ldots \ldots$

4.1 Physical properties of the fluids used in simulation. . . . . . . . . 54

4.2 The spacial step size $\Delta x^{p}$ in [nm] as a function of relaxation times $\tau_{1}$

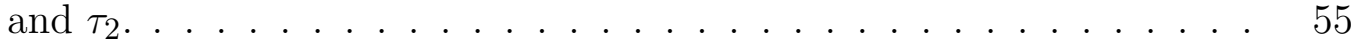

5.1 The dimensions of the T-junctions used for passive separation as Fig. 1.3 shows. . . . . . . . . . . . . . . . . . . 


\section{List of Figures}

1.1 Multisteps separator involves different forms of separation processes .

1.2 The expected effect of surface wettability change on contact angles in preparation of separation of two immiscible fluids. . . . . . . . . .

1.3 The geometry and wetting conditions of the channel to be used for passive separation in two-phase flows. . . . . . . . . . . . .

1.4 The geometry and wetting conditions of the channel to be used for passive separation in single phase two components flows. . . . . . .

1.5 The development of concentration boundary layer due to surface effect for (a) Even wettability. (b) Uneven wettability. . . . . . . . . .

3.1 The D3q27 [top] and D2q9 [bottom] lattice sets used in this work for immiscible and miscible cases respectively. . . . . . . . . . . . . .

3.2 Streaming process of a D2Q9 velocity set (a) the pre-streaming condition (b) the post streaming condition. . . . . . . . . . . . . .

3.3 The Bounce Back (BB) process used to impose the no slip boundary

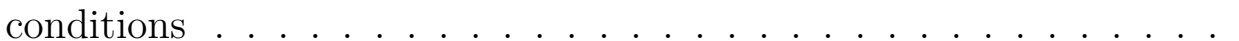

3.4 A fluid node adjacent to a solid surface interacting with another fluid through blue vectors and the solid nodes through the red links. . . . .

3.5 The multirange lattice with the order of isotropy identifying the extra nodes of the interaction forces . . . . . . . . . . . . . . .

3.6 Static balance of interfacial tensions of a liquid drop on a solid surface

3.7 A snapshot of contact angle measurement using ImageJ software. The result Theta $\mathrm{C}$ is the quantity of interest. We subtract it from $180^{\circ}$ to calculate the contact angle from inside. . . . . . . . . . . . . 
3.8 Density profiles of two immiscible fluids at $G_{12}=6.0, G_{1-1}=-0.75$ and different surface interactions coefficients. Fluid densities are $\rho_{1}=1.0$, and $\rho_{2}=0.77 \ldots \ldots \ldots \ldots$. . . . . . . . . . . . . 46

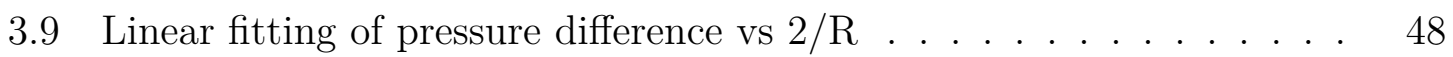

3.10 (a)Velocity profile from both N-S exact solution and LBM binary mixture Shan-Chen model when $\frac{d p}{d x}=0.00001, \nu_{1}=\nu_{2}=0.017, \rho_{1}=\rho_{2}=$ 1.0, $W=32$ lattices. (b) Relative local error between velocities in (a). The maximum relative error is less than $17 \%$. . . . . . . . . . .

3.11 The evolution of neck thickness of a droplet of density $\rho_{1}=1.0$ and viscosity $\nu_{1}=0.33$ surrounded by a fluid of density $\rho_{2}=0.77$ and viscosity $\nu_{2}=0.33$ under even wetting conditions $\theta_{\text {upper }}=\theta_{\text {lower }}=173.2 \pm 7.1$ at capillary number $C a=0.0063$ corresponding to Reynolds number $R e=0.381$. The initial droplet length is $L_{0}=3 W$ while the final

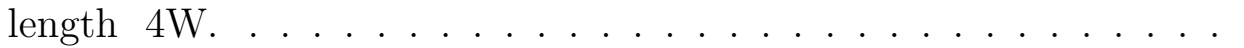

3.12 Neck width evolution with time: a comparison between present work and the literature. . . . . . . . . . . . . . . . . . .

4.1 Scaled densities of a binary fluid mixture components at $G_{1 s}=0.5$ (low affinity), $G_{2 s}=-0.5$ (high affinity) and cross interaction parameter, $G_{12}=1.00$ when $\tau_{1}=\tau_{2}=0.75 .(\mathrm{a})-\rho_{1}^{*}=\frac{r h o_{1}}{r h o_{1}^{i n i t}}$ and $(\mathrm{b})-\rho_{2}^{*}=\frac{\rho_{2}}{\rho_{2}^{i n i t}} . \rho_{1}^{*}$ decreases near the walls due to repulsion while $\rho_{2}^{*}$ increases near the walls due to attraction. The wall effect does not exceed 20[nm] and the densities far away from the walls are constant. . . . . . . . .

4.2 Effect of cross interaction parameter, $G_{12}$, on densities of both fluids at $G_{1 s}=0.5$, and $G_{2 s}=-0.5$ when $\tau_{1}=\tau_{2}=0.75$. (a) $-\rho_{1}^{*}=\frac{r h o_{1}}{r h o_{1}^{i n i t}}$ and (b) $-\rho_{2}^{*}=\frac{\rho_{2}}{\rho_{2}^{i n i t}}$ in the middle of channel. Increasing $G_{12}$ reduces the dispersion of $\rho_{1}^{*}$ and concentrates $\rho_{2}^{*}$ near the walls. . . . . . . .

4.3 Scaled densities of both fluids at $G_{1 s}=1.0, G_{2 s}=0.0$, and $G_{12}=0.5$ when $\tau_{1}=\tau_{2}=0.75$. (a) $-\rho_{1}^{*}=\frac{r h o_{1}}{r h o_{1}^{i n i t}}$ and $(\mathrm{b})-\rho_{2}^{*}=\frac{\rho_{2}}{\rho_{2}^{i n i t}} . \ldots$.

4.4 Effect of Solid-Fluid interaction parameter, $G_{1 s}$, on densities of both fluids in the middle of channel at $G_{2 s}=0.0$, and $G_{12}=0.5$ when $\tau_{1}=$ $\tau_{2}=0.75 . \quad(\mathrm{a})-\rho_{1}^{*}=\frac{r h o_{1}}{r h o_{1}^{i n i t}}$ and $(\mathrm{b})-\rho_{2}^{*}=\frac{\rho_{2}}{\rho_{2}^{\text {init }}} \cdot \rho_{1}$ is directly affected near the wall while $\rho_{2}$ is slightly changed due to $G_{1 s}$ change. . . . . . 
4.5 Scaled densities of both fluids at $G_{2 s}=1.0, G_{1 s}=0.0$, and $G_{12}=0.5$

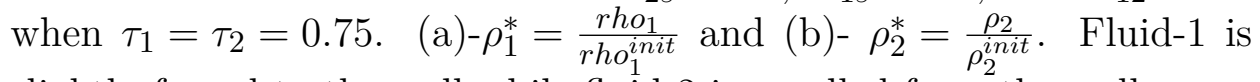
slightly forced to the wall while fluid-2 is repelled from the wall. .

4.6 Effect of Solid-Fluid interaction parameter, $G_{2 s}$, on densities of both fluids in the middle of channel at $G_{1 s}=0.0$, and $G_{12}=0.5$ when $\tau_{1}=$ $\tau_{2}=0.75$. (a) $-\rho_{1}^{*}=\frac{r h o_{1}}{r h o_{1}^{\text {init }}}$ and (b) $-\rho_{2}^{*}=\frac{\rho_{2}}{\rho_{2}^{\text {init }}}$. Fluid-1 is slightly affected by $G_{2 s}$ while fluid-2 is noticeably impacted near the wall.

4.7 Scaled densities of both fluids at relaxation time $\tau_{1}=1.0$ when $\tau_{2}=$ $0.75, G_{12}=0.5, G_{1 s}=0.5$ and $G_{2 s}=-0.5$.(a) $-\rho_{1}^{*}=\frac{r h o_{1}}{r h o_{1}^{\text {init }}}$ and (b)- $\rho_{2}^{*}=$ $\frac{\rho_{2}}{\rho_{2}^{i n i t}}$.

4.8 Effect of relaxation time of fluid- $1, \tau_{1}$, on densities of both fluids in the middle of channel at $\tau_{2}=0.75, G_{12}=0.5, G_{1 s}=0.5$ and $G_{2 s}=-0.5$. (a) $-\rho_{1}^{*}=\frac{r h o_{1}}{r h o_{1}^{\text {init }}}$ and (b)- $\rho_{2}^{*}=\frac{\rho_{2}}{\rho_{2}^{\text {init }}}$. The length scale over which the density varies is extended when $\tau_{1}$ is decreased. . . . . . . . . . . . .

4.9 Scaled densities of both fluids of both fluids at relaxation time, $\tau_{2}=1.0$ when $\tau_{1}=0.75, G_{12}=0.5, G_{1 s}=0.5$ and $G_{2 s}=-0.5$. (a) $-\rho_{1}^{*}=\frac{r h o_{1}}{r h o_{1}^{i n i t}}$ and $(\mathrm{b})-\rho_{2}^{*}=\frac{\rho_{2}}{\rho_{2}^{i n i t}} \ldots \ldots \ldots \ldots \ldots \ldots \ldots$

4.10 Effect of relaxation time of fluid-2, $\tau_{2}$, on densities of both fluids in the middle of channel at $\tau_{1}=0.75, G_{12}=0.5, G_{1 s}=0.5$ and $G_{2 s}=-0.5$. (a) $-\rho_{1}^{*}=\frac{r h o_{1}}{r h o_{1}^{\text {init }}}$ and (b)- $\rho_{2}^{*}=\frac{\rho_{2}}{\rho_{2}^{\text {init }}}$. The length scale over which the density varies is extended when $\tau_{2}$ is decreased. . . . . . . . . .

4.11 Scaled densities of both fluids((a),(c),(e)- $\rho_{1}^{*}=\frac{r h o_{1}}{r h o_{1}^{\text {init }}}$ and (b), (d), (f)$\left.\rho_{2}^{*}=\frac{\rho_{2}}{\rho_{2}^{\text {init }}}\right)$ for different channel heights when $\tau_{1}=\tau_{2}=0.75, G_{12}=$ $0.5, G_{1 s}=0.5$ and $G_{2 s}=-0.5$.

4.12 The effect of changing the height of channel by reducing the number of cells on the scaled species densities $\left((\mathrm{a})-\rho_{1}^{*}=\frac{r h o_{1}}{r h o_{1}^{i n i t}}\right.$ and $\left.(\mathrm{b})-\rho_{2}^{*}=\frac{\rho_{2}}{\rho_{2}^{\text {init }}}\right)$ when $\tau_{1}=\tau_{2}=0.75, G_{12}=0.5, G_{1 s}=0.5$ and $G_{2 s}=-0.5$. . . . .

4.13 Scaled densities of both fluids ( $\rho_{1}$ left and $\rho_{2}$ right) at different channel heights under uneven wetting conditions where top has: $G_{1 s}=-1.0$ and $G_{2 s}=1.0$ and bottom: $G_{1 s}=1.0$ and $G_{2 s}=-1.0$ with fixed $\tau_{1}=$ $\tau_{2}=0.75, G_{12}=0.5 . \ldots \ldots \ldots \ldots \ldots \ldots \ldots \ldots \ldots \ldots \ldots$ 
4.14 Profiles of scaled densities of both fluids ((a)- $\rho_{1}^{*}=\frac{r h o_{1}}{r h o_{1}^{\text {init }}}$ and (b)$\left.\rho_{2}^{*}=\frac{\rho_{2}}{\rho_{2}^{i n i t}}\right)$ at the middle of channel for heights under uneven wetting conditions where top has: $G_{1 s}=-1.0$ and $G_{2 s}=1.0$ and bottom: $G_{1 s}=1.0$ and $G_{2 s}=-1.0$ with fixed $\tau_{1}=\tau_{2}=0.75, G_{12}=0.5 . \ldots$

4.15 Contours of scaled densities ( $\rho_{1}$ left and $\rho_{2}$ right) for variable horizontal spacing $S_{x}=\frac{1}{6}, \frac{1}{4}, \frac{1}{2}$ and $S_{y}=\frac{1}{2}$ with uneven wetting conditions where top has: $G_{1 s}=-1.0$ and $G_{2 s}=1.0$ and bottom: $G_{1 s}=1.0$ and $G_{2 s}=$ -1.0 with fixed $\tau_{1}=\tau_{2}=0.75, G_{12}=1.0 . \ldots$. . . . . . . . .

4.16 Evolution with time of scaled densities profiles ( $\rho_{1}$ left and $\rho_{2}$ right) for variable horizontal spacing $S_{x}=\frac{1}{6}, \frac{1}{4}, \frac{1}{2}$ and $S_{y}=\frac{1}{2}$ with uneven wetting conditions where top has: $G_{1 s}=-1.0$ and $G_{2 s}=1.0$ and bottom: $G_{1 s}=$ 1.0 and $G_{2 s}=-1.0$ with fixed $\tau_{1}=\tau_{2}=0.75, G_{12}=1.0 . \quad$. . . . . .

4.17 The steady state profiles of the scaled densities $\left((\mathrm{a})-\rho_{1}^{*}=\frac{r h o_{1}}{r h o_{1}^{\text {init }}}\right.$ and (b) $\left.-\rho_{2}^{*}=\frac{\rho_{2}}{\rho_{2}^{\text {init }}}\right)$ in a partially patterned channel for variable $S_{x}$ and fixed $S_{y}$ with uneven wetting conditions where top has: $G_{1 s}=-1.0$ and $G_{2 s}=1.0$ and bottom: $G_{1 s}=1.0$ and $G_{2 s}=-1.0$ with fixed $\tau_{1}=$ $\tau_{2}=0.75, G_{12}=1.0$. Periodic inlet and outlet conditions are prescribed.

4.18 The steady state profiles of the scaled densities $\left((\mathrm{a})-\rho_{1}^{*}=\frac{r h o_{1}}{r h o_{1}^{i n i t}}\right.$ and (b) $\left.-\rho_{2}^{*}=\frac{\rho_{2}}{\rho_{2}^{i n i t}}\right)$ in a partially patterned channel for variable $S_{y}$ and fixed $S_{x}$ with uneven wetting conditions where top has: $G_{1 s}=-1.0$ and $G_{2 s}=1.0$ and bottom: $G_{1 s}=1.0$ and $G_{2 s}=-1.0$ with fixed $\tau_{1}=$ $\tau_{2}=0.75, G_{12}=1.0$. Periodic inlet and outlet conditions are prescribed.

4.19 Contours of scaled densities ( $\rho_{1}$ left and $\rho_{2}$ right) in a partially patterned channel with fixed spacing $S_{x}=\frac{1}{3}$ and $S_{y}=\frac{1}{3}$ under uneven wetting conditions where top has: $G_{1 s}=-1.0$ and $G_{2 s}=1.0$ and bottom: $G_{1 s}=1.0$ and $G_{2 s}=-1.0$ with fixed $\tau_{1}=\tau_{2}=0.75, G_{12}=1.0$. Periodic inlet and outlet conditions are prescribed and the forcing term is $F_{x}=5 \times 10^{-4}$.

4.20 Scaled densities ( $\rho_{1}$ left and $\rho_{2}$ right) profiles across and along the channel in a partially patterned channel with fixed spacing $S_{x}=\frac{1}{3}$ and $S_{y}=\frac{1}{3}$ under uneven wetting conditions where top has: $G_{1 s}=-1.0$ and $G_{2 s}=1.0$ and bottom: $G_{1 s}=1.0$ and $G_{2 s}=-1.0$ with fixed $\tau_{1}=$ $\tau_{2}=0.75, G_{12}=1.0$. Periodic Inlet and outlet conditions are prescribed and the forcing term is $F_{x}=5 \times 10^{-4} \ldots \ldots \ldots$ 
4.21 Contours of scaled densities ( $\rho_{1}$ left and $\rho_{2}$ right) in a partially patterned channel with fixed spacing $S_{x}=\frac{1}{3}$ and $S_{y}=\frac{1}{3}$ under uneven wetting conditions where top has: $G_{1 s}=-1.0$ and $G_{2 s}=1.0$ and bottom: $G_{1 s}=1.0$ and $G_{2 s}=-1.0$ with fixed $\tau_{1}=\tau_{2}=0.75, G_{12}=1.0$. Inlet densities and outlet velocity are Dirichlet. . . . . . . . . . . .

4.22 Scaled densities $\left((\mathrm{a})-\rho_{1}^{*}=\frac{r h o_{1}}{r h o_{1}^{i n i t}}\right.$ and $\left.(\mathrm{b})-\rho_{2}^{*}=\frac{\rho_{2}}{\rho_{2}^{\text {init }}}\right)$ profiles along the channel in a partially patterned channel with fixed spacing $S_{x}=\frac{1}{3}$ and $S_{y}=\frac{1}{3}$ under uneven wetting conditions where top has: $G_{1 s}=-1.0$ and $G_{2 s}=1.0$ and bottom: $G_{1 s}=1.0$ and $G_{2 s}=-1.0$ with fixed $\tau_{1}=$ $\tau_{2}=0.75, G_{12}=1.0$. Inlet densities and outlet velocity are Dirichlet. .

4.23 Dimensionless velocity profiles in a partially patterned channel with fixed spacing $S_{x}=\frac{1}{3}$ and $S_{y}=\frac{1}{3}$ under uneven wetting conditions where top has: $G_{1 s}=-1.0$ and $G_{2 s}=1.0$ and bottom: $G_{1 s}=1.0$ and $G_{2 s}=$ -1.0 with fixed $\tau_{1}=\tau_{2}=0.75, G_{12}=1.0$. Inlet densities and outlet velocity are Dirichlet. . . . . . . . . . . . . . . .

4.24 Number of solid posts columns on pressure gradient and flow rate at $S_{x}=\frac{1}{3}$ and $S_{y}=\frac{1}{3}$ under uneven wetting conditions where top has: $G_{1 s}=-1.0$ and $G_{2 s}=1.0$ and bottom: $G_{1 s}=1.0$ and $G_{2 s}=-1.0$ with fixed $\tau_{1}=\tau_{2}=0.75, G_{12}=1.0$. Inlet densities and outlet velocity are Dirichlet. . . . . . . . . . . . . . . . . .

5.1 Initialization of a droplet of density $\rho_{1}=1.0$ and viscosity $\nu_{1}=0.33$ surrounded by a fluid of density $\rho_{2}=0.77$ and viscosity $\nu_{2}=0.33$ when $\Delta \theta=30$. Dimensions in $[\mu m]$ and each time step is $31.622[\mathrm{~ns}]$. . . .

5.2 Initialization of a droplet of density $\rho_{1}=1.0$ and viscosity $\nu_{1}=0.33$ surrounded by a fluid of density $\rho_{2}=0.77$ and viscosity $\nu_{2}=0.33$ when $\Delta \theta=53$. Dimensions in $[\mu m]$ and each time step is $31.622[\mathrm{~ns}]$. . . .

5.3 Initialization of a droplet of density $\rho_{1}=1.0$ and viscosity $\nu_{1}=0.33$ surrounded by a fluid of density $\rho_{2}=0.77$ and viscosity $\nu_{2}=0.33$ when $\Delta \theta=70$.Dimensions in $[\mu m]$ and each time step is $31.622[\mathrm{~ns}] . .$.

5.4 Initialization of a droplet of density $\rho_{1}=1.0$ and viscosity $\nu_{1}=0.33$ surrounded by a fluid of density $\rho_{2}=0.77$ and viscosity $\nu_{2}=0.33$ when $\Delta \theta=78$. Dimensions in $[\mu m]$ and each time step is $31.622[\mathrm{~ns}]$. . . .

5.5 Initialization of a droplet of density $\rho_{1}=1.0$ and viscosity $\nu_{1}=0.33$ surrounded by a fluid of density $\rho_{2}=0.77$ and viscosity $\nu_{2}=0.33$ when $\Delta \theta=90$. Dimensions in $[\mu m]$ and each time step is $31.622[\mathrm{~ns}]$. . . . . 
5.6 Initialization of a droplet of density $\rho_{1}=1.0$ and viscosity $\nu_{1}=0.33$ surrounded by a fluid of density $\rho_{2}=0.77$ and viscosity $\nu_{2}=0.33$ when $\Delta \theta=104$. Dimensions in $[\mu m]$ and each time step is $31.622[\mathrm{~ns}]$. . .

5.7 Initialization of a droplet of density $\rho_{1}=1.0$ and viscosity $\nu_{1}=0.33$ surrounded by a fluid of density $\rho_{2}=0.77$ and viscosity $\nu_{2}=0.33$ when $\Delta \theta=130$. Dimensions in $[\mu m]$ and each time step is $31.622[\mathrm{~ns}]$. . .

5.8 Hydrodynamic response of a droplet of density $\rho_{1}=1.0$ and viscosity $\nu_{1}=0.33$ surrounded by a fluid of density $\rho_{2}=0.77$ and viscosity $\nu_{2}=$ 0.33 when $\Delta \theta=30$ at capillary number $C a=0.029$ corresponding to Reynolds number $R e=0.455$. Dimensions in $[\mu \mathrm{m}]$ and each time step

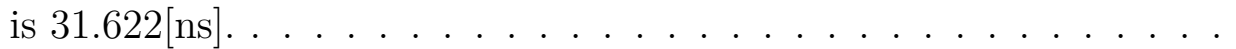

5.9 Hydrodynamic response of a droplet of density $\rho_{1}=1.0$ and viscosity $\nu_{1}=0.33$ surrounded by a fluid of density $\rho_{2}=0.77$ and viscosity $\nu_{2}=$ 0.33 when $\Delta \theta=53$ at capillary number $C a=0.029$ corresponding to Reynolds number $R e=0.455$. Dimensions in $[\mu m]$ and each time step

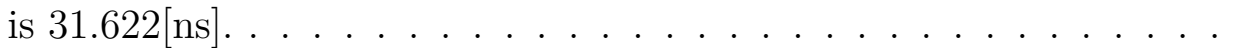

5.10 Hydrodynamic response of a droplet of density $\rho_{1}=1.0$ and viscosity $\nu_{1}=0.33$ surrounded by a fluid of density $\rho_{2}=0.77$ and viscosity $\nu_{2}=$ 0.33 when $\Delta \theta=70$ at capillary number $C a=0.029$ corresponding to Reynolds number $R e=0.455$. Dimensions in $[\mu m]$ and each time step

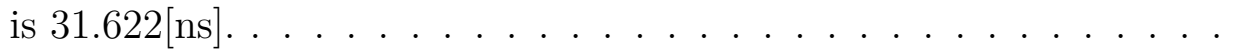

5.11 Hydrodynamic response of a droplet of density $\rho_{1}=1.0$ and viscosity $\nu_{1}=0.33$ surrounded by a fluid of density $\rho_{2}=0.77$ and viscosity $\nu_{2}=$ 0.33 when $\Delta \theta=78$ at capillary number $C a=0.029$ corresponding to Reynolds number $R e=0.455$. Dimensions in $[\mu m]$ and each time step

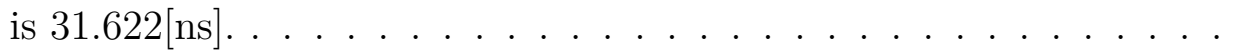

5.12 Hydrodynamic response of a droplet of density $\rho_{1}=1.0$ and viscosity $\nu_{1}=0.33$ surrounded by a fluid of density $\rho_{2}=0.77$ and viscosity $\nu_{2}=$ 0.33 when $\Delta \theta=90$ at capillary number $C a=0.029$ corresponding to Reynolds number $R e=0.455$. Dimensions in $[\mu m]$ and each time step

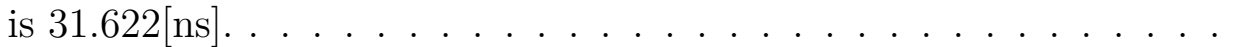

5.13 Hydrodynamic response of a droplet of density $\rho_{1}=1.0$ and viscosity $\nu_{1}=0.33$ surrounded by a fluid of density $\rho_{2}=0.77$ and viscosity $\nu_{2}=$ 0.33 when $\Delta \theta=104$ at capillary number $C a=0.029$ corresponding to Reynolds number $R e=0.455$. Dimensions in $[\mu m]$ and each time step is $31.622[\mathrm{~ns}] \ldots \ldots$. . . . . . . . . . . . . . . . . . . . . . . . . . . 110 
5.14 Hydrodynamic response of a droplet of density $\rho_{1}=1.0$ and viscosity $\nu_{1}=0.33$ surrounded by a fluid of density $\rho_{2}=0.77$ and viscosity $\nu_{2}=$ 0.33 when $\Delta \theta=130$ at capillary number $C a=0.029$ corresponding to Reynolds number $R e=0.455$. Dimensions in $[\mu m]$ and each time step is $31.622[\mathrm{~ns}] \ldots \ldots \ldots \ldots$. . . . . . . . . . . . . . . . . . . . 111

5.15 Effect of the initial droplet length on the droplet break up when $\theta_{\text {upper }}=$ $95.2 \pm 0.4$ and $\theta_{\text {lower }}=173.2 \pm 7.1$ at capillary number $\mathrm{Ca}=0.029$. Dimensions in $[\mu \mathrm{m}]$ and each time step is $31.622[\mathrm{~ns}] . \ldots . . . . .113$

5.16 Effect of capillary number on the droplet break up when $\theta_{\text {upper }}=95.2 \pm$ 0.4 and $\theta_{\text {lower }}=173.2 \pm 7.1$ at a droplet length $L_{0}=4 W$. Dimensions in $[\mu m]$ and each time step is $31.622[\mathrm{~ns}] . \ldots \ldots$. . . . . . . . . 114

5.17 Break up and no-break up regimes under different capillary numbers

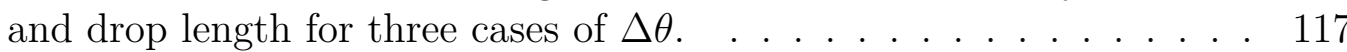

5.18 The Ca- $L_{0}$ diagrams for drop flows in T-junction from Jullein et al's experimental study . . . . . . . . . . . . . . 118

5.19 The Ca- $L_{0}$ diagrams for water (viscosity $\mu=0.894 \mathrm{mPas}$ ) droplets dispersed in Hexadecane+SPAN80 (viscosity $\mu=8.0 \mathrm{mPas}$ ) continuous flow in T-junction from Link et al's experimental study . . . . . . . . 119

5.20 Effect of the viscosity ratio $\lambda$ on break up and no-break up regimes for different capillary numbers and drop length. . . . . . . . . . . . . . . 121

5.21 Effect of the channel aspect ratio AR on break up and no-break up regimes for different capillary numbers and drop length. . . . . . . . 124

5.22 Effect of the side to main channel width ratio AR on break up and no-break up regimes for different capillary numbers and drop length. . 126

6.1 A suggested T-junction with tilted side channels to reduce the difference in wetting conditions required for two-phase flow separation. . . 131 


\section{Chapter 1}

\section{Introduction}

Separations are a key component in a wide range of chemical and biochemical applications [1]. When separation is achieved, a new mass distribution between two-phases is obtained. This distribution change is accomplished either by manipulation of the original phase equilibrium or by generation of a second phase with a different component distribution [2]. Separation processes involve phase creation or addition, see Fig1.1, which can be facilitated by including a barrier, solid agent, force field, or gradient with the aid of mass and/or energy separation agents [3]. Mass separation agents are vital for many separation processes and are introduced as solid, liquid, or gas. Energy separation agents include heat as well as external magnetic and electric fields. Heat is employed extensively in distillation processes [2].

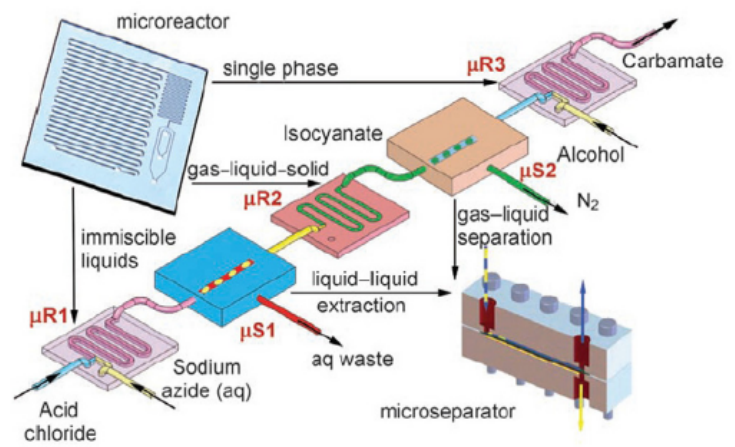

Figure 1.1: Multisteps separator involves different forms of separation processes[4]. 
The addition of separation agents in traditional separation processes increases costs associated with the process due to the expensive energy and mass agents. For example, to remove water from ethanol, a huge amount of heat is implemented to dehydrate ethanol by the means of pervaporation to raise the temperature of the mixture to $80^{\circ} \mathrm{C}[5]$. While mass agents such as zeolite [6] and palladium [7] used for membranes are very expensive to fabricate which makes them unfeasible for large scale.

While separation processes are commonplace throughout industry and the procedures for the separation process are well established, developing new separation techniques that limit energy consumption or reduce the dependence on mass agents are highly desirable both from an economic (less cost) and environmental (less waste) standpoint. Recent advances in microfabrication techniques have opened the possibility of chemical processing on the microscale.

When separation processes are miniaturized, the mass transfer performance is improved due to the enhanced gradients and high surface-to-volume ratios [4]. Therefore, the use of microseparators may remove the mass transfer limitations resulting in faster transfer rates. By following this approach, thermodynamic equilibrium which determines the separation efficiency is possible to reach rapidly [1]. Developing separation processes at the microscale requires knowledge of fluid behavior at length scales not typically found in current separation techniques.

Microfluidics is the research area related to flows that are confined in small systems of characteristic lengths around the order of several hundred microns. It includes the manner, rigorous control, and handling of flows at submillimeter scale. Many fields such as engineering, physics, chemistry, microtechnology, and biotechnology are interconnected by the design and modeling of entities within this scale [8]. With further technological advances, microfluidics will evolve to flows in channels of inner diame- 
ter of order of $1 \mu \mathrm{m}$ [9]. Coupled with nanoscale detectors or controllers, microscale networks will bridge the micro and nanoscales [10].

To avoid ambiguity associated with device size and the classification of microfluidics, it is necessary to adopt the scale which impacts flow behavior rather than size itself. This requires the application of scaling laws to describe the aimed performance [11]. Scaling laws indicate that microscale physics is dominated by surface effects [12]. Consequently, the surface forces are more significant than their body counterparts [13]. This dominance cannot be evaluated by implementing the basic laws of proportionality to traditional hydraulic systems. Moreover, some canonical forms of the classical fluid mechanics are basically not applicable in microfluidics [9].

To understand the differences in system performance due to reduced dimensions, physical quantities have to be connected to the characteristic length $l$ of the object or the entity to be studied [8]. Examples of some of these quantities are shown in Table 1.1. It is apparent from the tabulated quantities that body forces, $F_{b} \sim l^{3}$, while the surface forces $F_{s} \sim l^{2}[8]$. Therefore, the ratio of surface to body forces $\sim l^{-1}$. That means the influence of body forces on system's dynamics diminishes as its size is scaled down. On the other hand, the length dependence of capillary and Van derWaals forces dictate their increased importance.

Both of these forces emerge due to short range interactions among substances' molecules leading to a variety of phenomena such as capillarity, sessile drops profiles, and the Plateau Rayleigh instability. These microscale forces manifest macroscopic effects [14] even though the extent of forces does not exceed a few nanometers [10] 
Table 1.1: Scaling laws for different physical quantities [10].

\begin{tabular}{ll}
\hline Quantity & Scaling law \\
\hline Intermolecular Van derWaals force & $l^{-7}$ \\
Density of Van derWaals force between interfaces & $l^{-3}$ \\
Time & $l^{0}$ \\
Capillary force & $l^{1}$ \\
Distance & $l^{1}$ \\
Flow velocity & $l^{1}$ \\
Thermal power transferred by conduction & $l^{1}$ \\
Electrostatic force & $l^{2}$ \\
Diffusion time & $l^{2}$ \\
Volume & $l^{3}$ \\
Mass & $l^{3}$ \\
Force of gravity & $l^{3}$ \\
Magnetic force with an exterior field & $l^{3}$ \\
Magnetic force without an exterior field & $l^{4}$ \\
Electrical motive power & $l^{3}$ \\
Centrifugal force & $l^{4}$ \\
\hline
\end{tabular}

At the microscale, fluid motion may be controlled by varying fluid surface interactions by appropriate patterning materials with different molecular interactions with a chosen fluid. Alternatively, separation processes which depend on the wetting characteristics of the neighboring surfaces may be implemented [15-17]. This separation mechanism differs significantly from conventional processes which depend on gravity [18] as the separation process is controlled by fluid-surface and fluid-fluid interactions associated with each distinct fluid pair [19].

\subsection{Objective of This Work}

This work explores the passive separation of immiscible and miscible fluid mixtures by controlling the interfacial forces between fluid components and microchannel surfaces. Two primary cases are examined. First we investigate two immiscible fluids in T-shaped microchannels with hydrophobic and hydrophilic surfaces. This variation 
in wettability and the resulting contact angle indicates which side of a microchannel a particular fluid will adhere to. Figure 1.2 shows the expected effect on contact angle for a hydrophilic and hydrophobic fluid in contact with a microchannel with a hydrophilic and hydrophobic side. The fluid with higher affinity to the wall will preferentially adhere to the hydrophilic side. If the microchannel contains a T-junction with matching wettability, (Figure 1.3), the two-phases may separate into the channel with greater wettability difference.

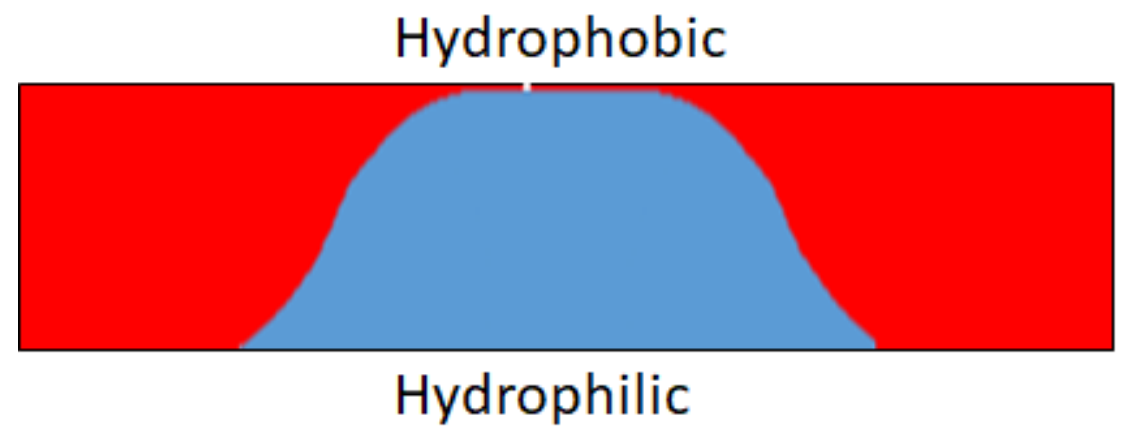

Figure 1.2: The expected effect of surface wettability change on contact angles in preparation of separation of two immiscible fluids.

Second, we study the separability of single phase two components fluid mixture in a two dimensional channel with uneven wetting conditions. Figure 1.4 provides the schematic of case 2. For this phase we consider the intermolecular interactions between the two liquid species and the intermolecular interaction with the surrounding surface. The fluid-solid interaction controls the affinity of each fluid component to the surface. The liquid component with a higher affinity should be attracted to the surface while the fluid component with a lower or opposite affinity should be repelled away from the boundary. The simultaneous attractions and repulsions experienced by fluid components must result in transverse components of momentum. Consequently, the concentration field of each species are affected by their corresponding momentum. 


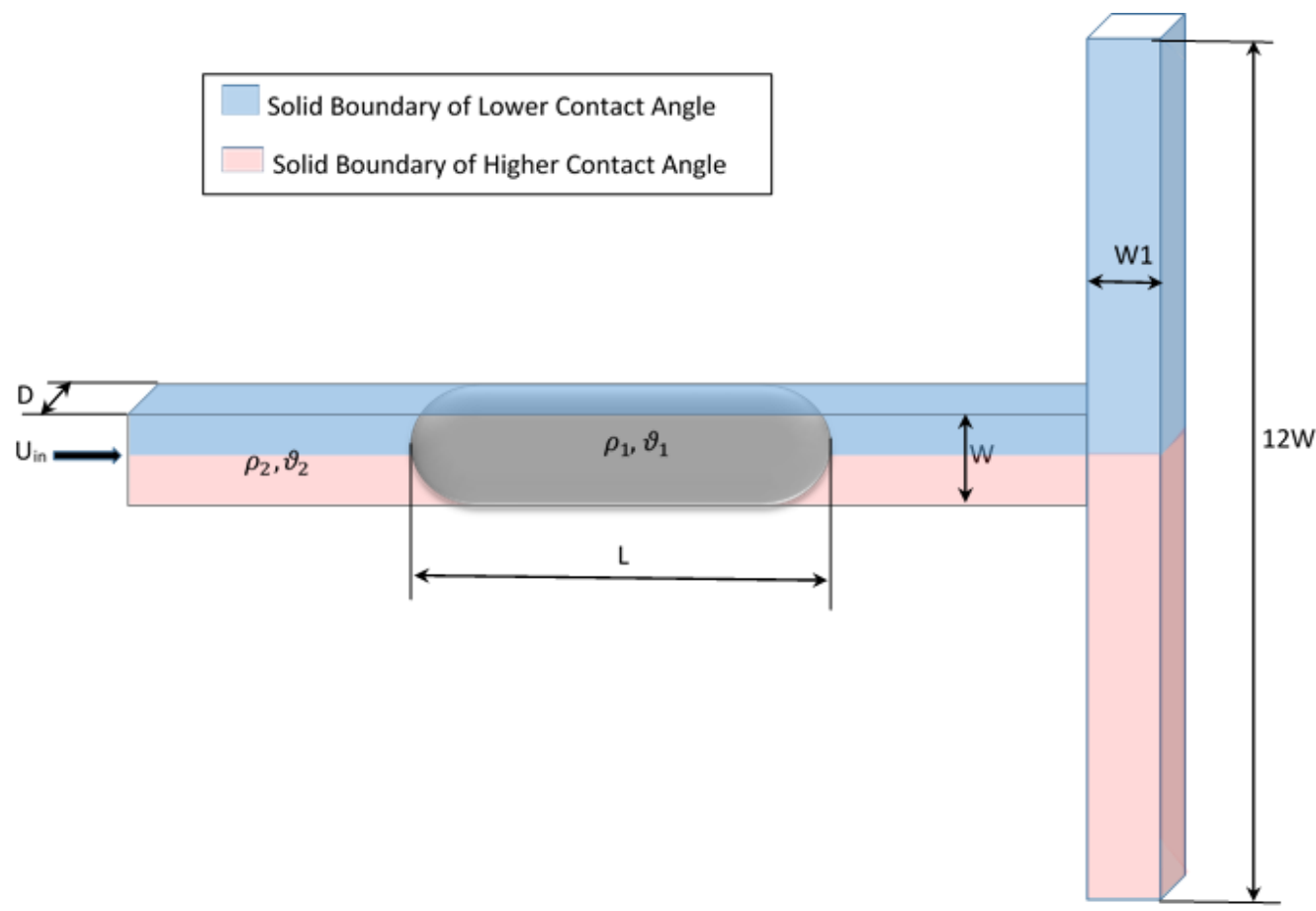

Figure 1.3: The geometry and wetting conditions of the channel to be used for passive separation in two-phase flows.

Ultimately, the component with higher affinity to the wall should concentrate near the wall. The actual concentration profile depends on the magnitudes and the signs of the interaction forces between the fluid components and the solid boundary. Figure 1.5 shows two possible concentration profiles that may develop.

The present study aims to quantify the effects of wetting conditions represented by the component interaction forces with solid walls under flow and no flow condition. Effects of the cross interaction parameter and the height of the channel are also considered. Additionally, we employ uneven wettability conditions to separate two immiscible fluids passively.

To the best of our knowledge, this work applies a novel approach to studying the separation of immiscible (case1) and miscible (case2) fluids. Specifically, the Lattice Boltzmann Method (LBM) is employed to simulate two-phase immiscible flow and 
Attraction Surface Effect

Repulsion Surface Effect

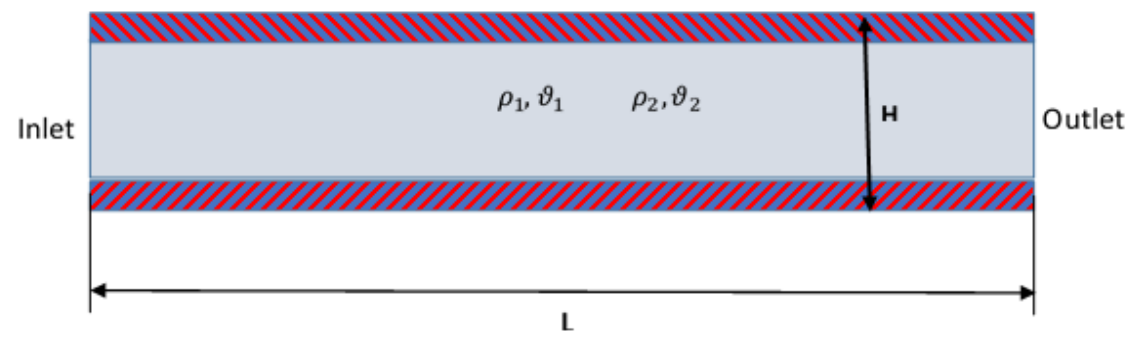

Figure 1.4: The geometry and wetting conditions of the channel to be used for passive separation in single phase two components flows.
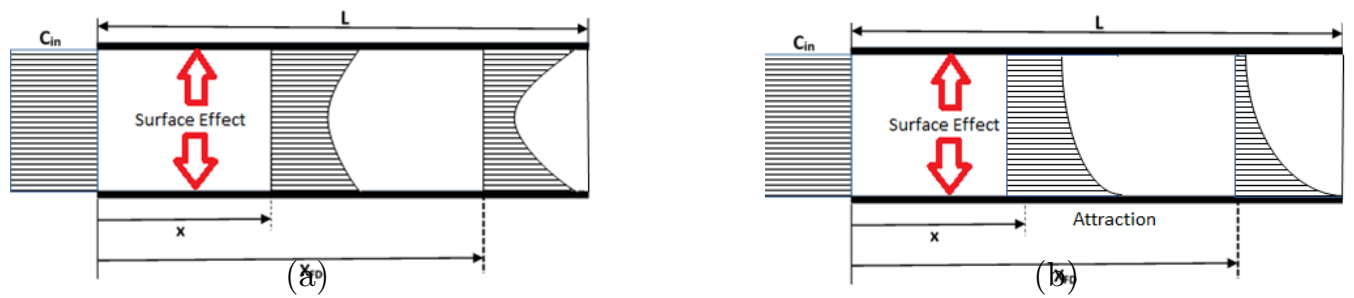

Figure 1.5: The development of concentration boundary layer due to surface effect for (a) Even wettability. (b) Uneven wettability.

one phase two component flow. The Shan-Chen LBM model is adapted to handle both fluid-fluid intermolecular interactions and fluid-solid intermolecular interactions. 


\section{Chapter 2}

\section{Literature Review}

In the next few sections we highlight the most relevant studies of separations in micro scale systems. These works are classified into: solid particles sorting, gasliquid separation, and liquid-liquid separation. We focus on the driving mechanism of separation, flow conditions, and the outcomes of each work. This review highlights the knowledge gap in the literature that this work seeks to fill.

\subsection{Particles Separation}

Go et al. were able to completely separate beads of different sizes, materials, and shapes using the inertia effect arising from the flow in a curved rectangular microchannel [20]. In their numerical analysis and experimental visualization, they examined non spherical nickel and spherical glass beads in a channel of $75 \mu \mathrm{m}$ height and $150 \mu m$ width with $200 \mu m$ curvature radius at different values of Dean's number. They observed that almost all the time small beads tend to move inward while the large beds are displaced outward when different beads of different materials are used. This is not necessarily the norm as small beads made from glass start to move outward together with the large ones when the Dean number reaches 28.87. They attributed that to a vertical component of fluid velocity which becomes significant at the corresponding value of Dean's number. In addition, the appropriate positioning 
of beads at the inlet has a remarkable influence on the separation efficiency.

The repulsive wall effect, which competes with the inertial lift force, was employed to achieve the encapsulation of single cells into picolitre drops [21]. The main purpose of the experimental work was to overcome the limitations set by Poisson's statistics by loading each drop with exactly on cell or particle by means of the inertial sorting in a rectangular microchannel of $27 \mu \mathrm{m}$ width, $52 \mu \mathrm{m}$ height, and $6 \mathrm{~cm}$ length. HL-60 (Human promyelocytic leukemia cells) and $9.9 \mu \mathrm{m}$ beads were used independently to examine the possibility that each drop would carry single or multiplies cells. The results revealed that the single to multiplies ratio was 30.9 for beads and 56.5 for cells. The flow rates needed to maintain self ordering were found to be $10 \mu \mathrm{L} / \mathrm{min}$ for beads and $13 \mu L /$ min for cells. Higher flow rates were found to have a slight effect on the encapsulation. The formation of multiplies was attributed to the particles aggregation emerging from the initial patching of particles or cells.

Matas et al. [22] studied the motion of buoyant suspensions traversing through small tubes by Poiseuille flow. Polystyrene spheres of mean diameters $425 \pm 25 \mu \mathrm{m}$ and $825 \pm 25 \mu m$ were injected to horizontal glass tubes of length $2.6 \mathrm{~m}$ and diameters $\mathrm{D}=8 \mathrm{~mm}$ and $\mathrm{D}=14 \mathrm{~mm}$ respectively. The suspending liquid was prepared by mixing $22 \%$ glycerol and $78 \%$ water to achieve the same density of suspensions. The liquid was injected such that Reynolds number does not exceed 2000 for both tubes. The onset of particle trains was observed when Reynolds number exceeds 100. The length of these trains started to increase with the increase of Reynolds number until reaching their peaks when $R e \sim 700$ and $R e \sim 900$ for large particle-large tube, and small particle-small tube and small particle-large tube consequently. The trains were noticed to travel along on the Segré-Silberberg annulus which becomes closer to the walls as the inertial effect become stronger. However, some particles were found to deviate from the trains due to the flattening they experienced during pumping which 
in sequence affected their inertial lift and mutual interaction with other particles.

A similar study was done numerically by Inamuro et al. [23] but using neutrally buoyant circular cylinders instead of spheres and two parallel walls instead of circular tubes. The Lattice Boltzmann method was employed to perform the computations and paired with Newton's law to configure the motion of cylinders from the stress fields that impose hydrodynamic forces on the suspensions. Single and two lines of cylinder were tested under the effect of Reynolds number: $12<R e<96$, the ratio of cylinder diameter to the channel width: $0.25 \leq D_{s} / D \leq 0.5$, and spacing in the direction of the stream to the channel width $0.75 \leq L / D \leq 2$. The cylinders were found to equilibrate on the Segre - Silberberg line. These equilibrium positions would approach the wall when Reynolds number increased and drift away toward the center when the ratio of cylinder diameter to the channel width increased.

For better understanding of the hydrodynamic interactions exchanged between two individual particles in shear flow, Yan et al. [24] implemented a numerical investigation of two cylindrical and particles in a Couette flow confined by two moving plates moving in opposite directions. They used Lattice Boltzmann method to establish the mass and momentum fields at finite particle Reynolds number, $R e_{p}$. The study focused on two main cases: either both particles are moving or one is fixed and the other is mobile. The trajectories of both particles were found to be a nontrivial function of initial positions of particles relative to the center line of the channel, the separation distance between particles, and particle Reynolds number. The behavior of the moving particle is affected by it's location with respect to the periodic image of the second particle. This behavior is represented by the reflecting or the limit cycle trajectories.

Paputsky et al. achieved complete separation of $7.32 \mu$ and $1.9 \mu$ particles using a spiral microchannel [25]. The helical geometry consisted of 5 turns rectangular cross 
section microchannel with $100 \mu$ width and $50 \mu$ height. The competition between inertial lift and viscous drag was employed to displace particles into two streams next to the external and internal walls depending on the suspensions sizes. The experiments were conducted under $R e \sim 10$ and $D e \leq 1.0$ where the passive separation was possible. In addition, the separation process including the channel design was modeled using a commercial package called CFD-ACE+. The Dean drag and the lift forces were found to increase proportionally with the particle size as $F_{D} \propto d_{p}$ and $F_{L} \propto d_{p}^{4}$ respectively. Therefore, the Dean force dominates for the $1.9 \mu$ while the lift force is stronger for the $7.32 \mu$ leading to entraining the smaller particles near the outer wall and the larger particles near the inner wall when $D e=0.94$.

Tian et al. [26] achieved a complete separation of polystyrene particles as well as biological cells at the microscale. They used the interfacial effect between Newtonian and viscoelastic fluids, made with the aid of elastomers, as a passive controller to sort microparticles according to their size. The basic idea was to employ the wall directed elastic effect, which resists the inertial lift effect toward the center, as a filter to pass the large particles and capture the smaller ones. The flow pattern used for this purpose was the sheath flow where the the viscoelastic fluid was injected at rate $0.3 m L / h$ in the center while the Newtonian fluid was injected peripherally at rate 1.2 to $3.0 \mathrm{~mL} / \mathrm{h}$. The separation device was a straight microchannel of dimensions $H \times W \times L=50 \mu \times 20 \mu \times 15 \mathrm{~mm}$ respectively. The increase of the elastic force by manipulating the concentration of the elastomer in the sheath fluid increased the separation distance where the large size particles, although the interface retards them, moved away toward the center. Thus, finding the optimum concentration was a must for complete separation. The flow rate appeared to be less effective on separation efficiency because the competing forces had the same order of proportionality with the flow velocity. The theoretical analysis of the forces acting on particles of different 
sizes confirmed the experimental investigation.

Oozeki et al. [27] experimentally characterized a curved micro-separator's performance in terms of the microparticles' cut size, separation sharpness, and feed pressure. An arc microchannel of dimensions $W \times H \times R=200 \mu m \times 150 \mu m \times 20 m m$ functioned as the active section where the arc angle ranged from $30^{\circ}$ to $180^{\circ}$. The slurry fed to the device consisted of acrylic particles $\left(1190 \mathrm{~kg} / \mathrm{m}^{3}\right)$ and deionized water $\left(996 \mathrm{~kg} / \mathrm{m}^{3}\right)$ with a surfactant. The particle sizes were 1.8, 5.0, 10, 15, 20, and $30 \mu \mathrm{m}$ respectively. The experiments ran under $\mathrm{Re}=300-1200$ and $\mathrm{De}=20-80$. The visualization of the slurry flows revealed that at $0.05 \%$ volume concentration small sizes $(1.5$ and $5.0 \mu m$ ) has the minimum separation efficiency for all values of Re due to their weak response to the lift effect. For 20 and $30 \mu m$ particles, the separation efficiency was the maximum for $\mathrm{Re}=600-900$ but degraded outside this range because of either the transition to turbulence at higher Re values or the insignificance of lift forces at low Re, which means that the higher velocity region does not move toward the outer wall. For the 10 and $15 \mu \mathrm{m}$ particles, the longer the arc the better the separation efficiency except for the $30^{\circ}$ channel that showed monotonic increase with Re. The concentration of the fed particles had an adverse effect on separation efficiency because the interactions between particles disperse them over the channel cross sectional area.

In case of biological cells separation, the confining solid walls may induce a damaging effect. To overcome this shortcoming, Wu et al. [28] developed a new device to separate human blood cells from Escherichia coli bacteria using soft inertial force focusing. They introduced sheath flows from both sides of the active channel to protect the sample and to displace cells according to acting inertial force. The resulting flow was curved and focused so that two streams of cells were apparent. The tested flow rate was up to $18 \mu \mathrm{l} / \mathrm{min}$ and provided up to 300 times enrichment for bacteria and throughput up to 68880 cells/s. 
Di Carlo [29] discussed the achievements in developing prototype inertial microfluidic systems for various applications and clarified the essential hydrodynamic effects exploited in modeling. He also suggested future promising directions to exploit fluid inertia on the microscale for optimal separation. The major effects that he considered very important for future theoretical models included: the finite-size of particles and how this affects local lift forces, variations of lift force and secondary flow configuration with Reynolds number, deformability- induced lift forces on particles and cells, interactions forces between particles and their arrangement, and effects of particles' topography.

\subsection{Liquid-Gas Separation}

Yue and colleagues [30] experimentally investigated the mass transfer characteristics of coincident gas-liquid flows in a rectangular microchannel of hydraulic diameter $667 \mu \mathrm{m}$. Their study focused on the adsorption of $\mathrm{CO}_{2}$ by water, $0.3 \mathrm{M} \mathrm{NaHCO} 3 /$ $0.3 \mathrm{M}$ Na2CO3 buffer solution and a $1 \mathrm{M} \mathrm{NaOH}$ solution. The superficial velocities of gas and liquid ranged from 0.7 to $13 \mathrm{~m} / \mathrm{s}$ and from 0.09 to $1.0 \mathrm{~m} / \mathrm{s}$ respectively. They found liquid side volumetric mass transfer coefficient and interfacial area to increase by increasing the superfacial gas velocity while the counterpart liquid velocity is constant; however, the liquid side mass transfer coefficient didn't respond significantly to preceding controls. The opposite condition is fixing the superfacial gas velocity and increasing the liquid counterpart enhanced the liquid side volumetric and regular mass transfer coefficients while the interfacial area did not change significantly. Finally, they concluded that using micro-contactors promotes the volumetric mass transfer coefficient and interfacial area of the liquid side 1 to 2 orders of magnitude compared to traditional contactors. 
Al-Rawashdeha and coworkers [31] studied the absorption of $\mathrm{CO}_{2}$ by $\mathrm{NaOH}$ liquid numerically and experimentally. The micro-reactors used in this study consisted of 16 to 120 straight microchannels connected in parallel and integrated to either a plate or a cylinder gas. The microchannel profile was analogous to elliptical section of $6.64 \mathrm{~cm}, 400 \mu \mathrm{m}$, and $1200 \mu \mathrm{m}$ length, minor and major diameters respectively. The liquid phase flow rate ranged from 1.6 to $20 \mathrm{ml} / \mathrm{min}$ and the gas chamber height ranged from 0.7 to $5.5 \mathrm{~mm}$. The results showed that the liquid flow rate had an adverse effect on the adsorption rate while the inaccuracy in the channel dimensions up to $11 \%$ for width and $6 \%$ for depth didn't impact the conversion process. The reduction of gas chamber height improved the gas side mass transfer. The increase of hydrophobicity by coating the microchannel's interior surface by Teflon reduced the gas absorption due to the decrease in the interfacial area of the liquid phase.

Constantinou and Gavriilidis [32] studied the absorption of carbon dioxide by sodium hydroxide liquid using microstructured metal mesh assembled between gas and liquid chambers. The micro-reactor consisted of eight $90 \times 5.48 \mathrm{~mm}$ micro channels on both side of the mesh with $200 \mu \mathrm{m}$ and $850 \mu \mathrm{m}$ depth for liquid and gas sides respectively. The flow conditions comprised 1.28 to $2.56 \mathrm{~mL} / \mathrm{min} \mathrm{NaOH}$ and 177 to $354 \mathrm{~mL} / \mathrm{min} \mathrm{CO}_{2}$ at $20^{\circ} \mathrm{C}$ which resulted maximum Reynolds number $R e=21$ and $R e=1.3$ for gas and liquid sides respectively. The results showed that $\mathrm{CO}_{2}$ absorption efficiency is directly proportional to liquid flow rate while it is inversely proportional to gas flow rate. They interpreted that by the decrease of residence time when the gas flow increased and the increased concentration of sodium hydroxide which would enhance the the reaction rate. The increase of mesh open area by 15 to $25 \%$ ameliorated the $\mathrm{CO}_{2}$ removal by 17.8 to $28 \%$.

Benefiting from Taylor flow, where gas bubbles are separated by liquid slugs and the large interfacial area promotes mass transfer, Shao and coauthors [33] investi- 
gated the removal of $\mathrm{CO}_{2}$ from $\mathrm{N}_{2} / \mathrm{CO}_{2}$ gas mixture numerically. They used $\mathrm{NaOH}$ and Water as liquid phase to extract the carbon dioxide inside a microchannels of hydraulic diameter $<1.0 \mathrm{~mm}$. The simulations ran under liquid Reynolds number, $5 \leq R e_{L} \leq 50$, atmospheric pressure and temperature $\mathrm{T}=298 \mathrm{~K}, 5.0 \% \mathrm{CO}_{2}$ volumetric concentration, and $0.2 \mathrm{M} \mathrm{NaOH}$. Three mass transfer scenarios: diffusion, advection, and advection with reaction were compared to study their effects on the absorption process. Increasing bubble velocity, $U_{b}$, improved mass transfer significantly until $U_{b}>0.05 \mathrm{~m} / \mathrm{s}$ where the circulation frequency of the liquid slug had the maximum impact on $\mathrm{CO}_{2}$ absorption. However, the absorption fraction, which denotes the percentage of $\mathrm{CO}_{2}$ transferred through the interface, and the utilization index, which determines the amount of $\mathrm{CO}_{2}$ absorbed per unit volume of $\mathrm{NaOH}$, had increased more significantly in the presence of chemical reaction than for advection alone. The elongation of gas bubbles from 0.25 to $5.0 \mathrm{~mm}$ lead to 300 to $858 \%$ increase of the utilization index in contrast to absorption fraction and mass transfer coefficient that decreased due to the excess $\mathrm{CO}_{2}$ on both sides of the interface. The increase of slug length had an adverse effect on the utilization index and little or no influence on the absorption fraction depending on the circulation frequency and its congruity with the gas bubble residence time. The effect of channel size was found to enhance the advective mass transfer characteristics in contrast to their reactive counterparts.

Separation of volatile components is not confined by absorption techniques. It is possible to recover organic solvents from aqueous solutions by microstripping. For example, Zanfir and coauthors [34] formulated and validated a two dimensional model for acetone stripping from isopropanol-acetone liquid with the aid of nitrogen as a sweeper gas. In addition, they investigated the hydrogenation of acetophenone and stripping the acetone at the same time. A microstructured mesh contactor was employed for this purpose. The contactor was $60 \mathrm{~mm}$ length $20 \mathrm{~mm}$ width; the gas 
and liquid passages were both of $200 \mu \mathrm{m}$ thickness and they were split by a $50 \mu \mathrm{m}$ thickness stainless steel 304 mesh with $23 \%$ porosity. It was evident that raising the liquid to gas flow rate ratio would strengthen the removal of acetone. It was also apparent that the stripping process reduced the liquid flow rate at the outlet because some isopropanol would evaporate with the acetone. It was beneficial to presaturate the nitrogen with isopropanol to overcome this issue. The effect of liquid flow rate on acetone removal was very slight and the concentration of acetone at the inlet had no impact.

Chasanis and coworkers [35] stripped the toluene from water by nitrogen gas using a porous sieve in a countercurrent flow contactor. They used 11 microchannels on both sides of sieve for contacting the liquid and gas phases. The range of liquid phase flow rate was 14 to $1390 \mathrm{~g} / \mathrm{h}$ while the gas phase flow rate ranged fom 2 to $106 \mathrm{l} / \mathrm{h}$. In this study, the trend contradicted normal microstripping studies; the separation performance was extremely sensitive to the liquid phase flow rate and independent of the nitrogen flow rate. Numerical analyses using ANSYS (CFX) were performed to validate the experimental data. The computations deviated from experiments especially at low liquid flow rate due to assumptions made to accelerate the simulation. Shape modification was implemented to improve separation efficiency by adding rectangular undulations to the liquid phase path. Increasing the amplitude of these undulations improved the stripping degree as the liquid phase velocity decreased. However, this improvement was constrained by the increase of pressure drop. The spacing of introduced objects had only a minor influence on toluene removal.

Moschou and his coleagues [36] investigated the separation of isopropyl-alcohol and toluene from a solvent layer by using Nitrogen as stripping agent. Two types of falling films microreactors, FFMR-Standard (120x76x40mm) and STACK-1x- FFMRLab(294x28x19mm), were studied numerically and semi-analytically and then com- 
pared to experimental data for validation. In both reactors the solvent was driven through an engraved channel by gravity effect and the stripping agents was in direct contact with the liquid phase. The gas and liquid mass transfer ranges were 50 to $1000 \mathrm{ml} / \mathrm{min}$ and 0.0001 to $10 \mathrm{ml} / \mathrm{min}$ respectively. The back side of the main plate in both reactors was heated isothermally using another liquid flowing concurrently. The two mass transfer models CFD using COMSOL Multiphysicss and semi-analytic using Sherwood correlations exhibited very good agreement when tested on a specific case of STACK-1x-FFMR-Lab. The results indicated that increasing gas volume flow rate enhanced the molar flux of solvent from the liquid to the gas side. The authors emphasized that Reynolds number had an appreciable impact on Sherwood correlation leading to an evident hydrodynamic variance between the standard FFMR and STACK-1x- FFMR-Lab.

Constantinou and his group [37] stripped acetone from water by nitrogen gas using glass/silicon and microchannel membrane contactors. In both reactors, the liquidgas interface is stabilized by a microfabricated structure to prevent breakthrough of either phase. In the first contactor, the microchannel was $35 \mathrm{~mm}$ long, $220 \mu \mathrm{m}$ wide, and $100 \mu m$ deep while the interface was controlled by $15 \mu m$ diameter micropillars. That resulted in a liquid layer of thickness $15 \mu \mathrm{m}$ and a gas channel of width $195 \mu \mathrm{m}$. The major section of the membrane contactor consisted of $90 \mathrm{~mm}$ length and 5.48 $\mathrm{mm}$ width chamber. The chamber was divided by a $20 \mu \mathrm{m}$ thick PTFE porous layer mounted on a $80 \mu m$ thick polypropylene porous layer. The resulting depths were $0.85 \mathrm{~mm}$ and $0.2 \mathrm{~mm}$ for gas and liquid sides respectively. In both contactors the liquid flow rate was kept constant at $0.001 \mathrm{ml} / \mathrm{min}$ and $0.13 \mathrm{ml} / \mathrm{min}$ for glass/silicon and PTFE type respectively. The corresponding gas flow rate ranged from 1.2 to 4.65 $\mathrm{ml} / \mathrm{min}$ and from 160 to $280 \mathrm{ml} / \mathrm{min}$ respectively. The experimental analysis of mass transfer for both cases was also compared to the analytic solution in terms of the 
dimensionless concentration. The results demonstrated that increasing gas flow rate enhanced the separation efficiency and the glass/silicon reactor was more effective than the PTFE based reactor.

Yang and coworkers [4] reviewed the microdistillation units comprehensively and they indicated that an efficient separation with short residence time are possible but require control of the temperature distribution, flow rates, pressure drop, and microchannel height. Microdistillation separation techniques work on the principle that each component of a mixture has different boiling points and consequently different vapor pressures [1]. Efficient distillation requires temperature elevation over different stages. Microscale devices used for distillation are characterized by high surface tension of the liquid phase and the hydrodynamics are driven by carrier-gas, vacuum, capillarity, or centrifugal forces[18].

\subsection{Liquid-Liquid Separation}

Kolehmainen and Turunen[15] manufactured a micro-separator to detach water from Shellsol D60 with tris (2-ethylhexyl) phosphate (TEHP) by coalescence due to surface forces. They injected these immiscible fluids between two plates made from PTFE, stainless steel and glass and had $15 \mathrm{~mm}$ width and 200mm length. The gap between these plates was either $100 \mu \mathrm{m}$ or $200 \mu \mathrm{m}$ and the flow rate tested was up to 480 $\mathrm{ml} / \mathrm{h}$ based on 1:1 aqueous to organic phase flow ratio. Because PTFE is highly hydrophobic, the water drops adhere to the plate made of Stainless steel or glass. That lead to formation of two adjacent streams, aqueous and organic, separated by an interface which facilitated the split into two outlets where each phase was withdrawn with high purity. The highest separation efficiency was possible for total flow rates under $180 \mathrm{ml} / \mathrm{h}$. The results indicated no significant difference when PTFE-glass 
coalescer was used compared to the PTFE-stainless steel one.

Castell et al. [38] manufactured a polytetrafluoroethylene (PTFE) microscale separator for water/chloroform segmented flow. The separation principle utilized was the difference in wettabilities of the two fluids with the fabrication material. The separator consisted of the main channel, $720 \mu m$ width and $600 \mu m$ depth, and an array of 140 side channels, $36 \mu m$ wide and $130 \mu m$ deep, to remove the organic segments. The total flow rate was up to $0.4 \mathrm{ml} / \mathrm{min}$ and the flow ratio was 1:1. The researchers had to control the pressure differences across the outlets to accomplish $100 \%$ separation efficiency. The maximum individual flow rate corresponding to the optimum separation was $0.2 \mathrm{ml} / \mathrm{min}$. The minimum number of effective side channels involved in the separation had a linear increase with the breakthrough limit of flow. The device was able to separate organic liquids loaded with $2.0 \mu \mathrm{m}$ polystyrene beads but with the corresponding increase in viscosity the pressure drop and the required number of side channels increased. The authors suggested that increasing the number of side channels, reducing their length, or changing their geometry would improve the separation under higher flow rates.

Scheiff et al. [39] experimentally separated water slugs from the Kerosene or paraffin oil using a hydrophobic microcapillary film (MCF) and hydrophilic steel needle. The plastic MCF, made of high density polyethylene, had a $630 \mu m$ inner diameter while the metal needle had either $0.82 \mathrm{~mm}$ or $0.51 \mathrm{~mm}$ inner diameter. To utilize the the hydrophilicity for separating the aqueous phase from the organic counterpart, the needle had to pierce the main channel with a specific penetration depth and side angle. The pierce position was located at $150 \mathrm{~mm}$ away from where the slugs were generated. The outlet of the main channel and the needle were connected to $0.5 \mathrm{~mm}$ and $1.0 \mathrm{~mm}$ inner diameter tubes of variable lengths to control pressure differences. Both phased were injected at $10 \mathrm{~mL} / \mathrm{h}$ and the volume fraction of water 
was fixed at 0.5. The penetration depth had a marginal influence on the degree of separation and the best piercing angle was $90^{\circ}$. The principal element of the separation process was the main outlet to the side-stream length ratio which had to be 12 to achieve best performance. The group developed mathematical model for the pressure drop across both outlets and they found both the surface tension ratio of the immiscible fluids and the size of the needle dictated the segregation efficiency.

Peronia et al. [40] developed a liquid-liquid extraction device based on segmented flow generated by micromixing. Ethyl ester and several methyl esters of fatty acids were present as analytes in water and extracted by organic solvents according to their hydrophobicicty. The research team formulated a theoretical model to predict the minimum detectable concentrations of analytes in terms of their hydrophobic coefficients $K_{o / w}$ and the aqueous to organic phase flow ratio $F_{a q} / F_{o r}$. The flow rates and flow ratios ranged from 0.5 to $6.0 \mathrm{~mL} / \mathrm{min}$ and 1 to $10 \mathrm{~mL} / \mathrm{min}$ respectively. The micro-extractor used for segmented flow had dimensions of $14.6 \mathrm{~cm} \times 7.3 \mathrm{~cm} \times$ $0.4 \mathrm{~cm}$ and an overall capacity $3.4 \mathrm{~mL}$. Split and reunion style 0.7 to $1.5 \mathrm{~mm}$ micro channels were engraved to promote the stability of the slugs. The extraction efficiency, defined as the percentage of analytes leaving the aqueous phase, was sensitive to the flow ratio. It was also evident that analytes with higher hydrophobicity were easy to extract implicating better separability. The organic slugs were withdrawn from the system after leaving the microextractor by a T-shape separator depending on the differences in affinity of the organic phase to the outlets.

Gaakeer and others [41] presented a separation device for water and n-heptane slug flow using a T-shape separator with hydrophobic and hydrophilic slits. The hydrophobic Teflon aisle and the hydrophilic glass counterpart had rectangular cross sections with height of 0.1 to $2 \mathrm{~mm}$, a length of $5 \mathrm{~mm}$, and a width of $10 \mathrm{~mm}$. The total flow rate range was 30 to $50 \mathrm{ml} / \mathrm{min}$ and the pressure drop accross both slits 
was less than $100 \mathrm{~Pa}$. The breakthrough of both liquids was avoided by controlling the capillary to hydraulic pressure ratio. This ratio was controlled by changing the heights of the Teflon and glass slits. The research group found that keeping the capillary to hydraulic pressure ratio $>2.0$ would prevent breakthrough from happening. Separation efficiency of $99.5 \%$ was achievable under the aforementioned constraints.

The possibility of water/ethanol mixture separation was demonstrated by molecular dynamics simulations using nano porous carbon membranes [5]. Namely carbon nano-tubes membranes, nano-porous graphene sheets, and multilayer graphene membranes were investigated with the open source code LAMMPS. The system consisted of two reservoirs, one filled with pure ethanol while the other full of a water/ethanol mixture. A carbon membrane connected the reservoirs and the was tank enclosed by graphene sheets from top and bottom to control the pressure drop. The side walls were assumed to be periodic. These membranes exhibited a counter-intuitive tendency to pass ethanol and block water despite the strong permeability of both liquids individually. This selective permeability suggests that carbon based membranes are semi-permeable to water with high affinity to ethanol when present as a mixture. The authors predict this permeability behavior should apply to a methanol/water mixture and hydrophobic, polymeric, and nano-porous layers.

Zhao and Li [42] employed the DC dielectrophoretic (DEP) effect to sort emulsified oil drops in an aqueous stream in a microchannel. They applied an electric field through side orifices of uneven dimensions. The oil droplet supply side orifices 860 $\mathrm{nm}$ wide and $290 \mathrm{~nm}$ deep while the carrier fluid supply side orifices were $125 \mu \mathrm{m}$ wide with the same depth of the main channel, $25 \mu \mathrm{m}$. The width of the main channel, suspending liquid inlet and the two outlets were $80 \mu m$ while the emulsion inlet was $20 \mu m$ wide. Sorting was achieved in two ways based on underlying principles of DEP force. In the first type, oil drops were displaced to their final trajectories according to 
size by the DEP force being proportional to third power of droplet radii. Thus, the $9 \mu \mathrm{m}$ and $14.5 \mu \mathrm{m}$ drops were sorted with $240 \mathrm{~V}$. The $7.5 \mu \mathrm{m}$ and $11 \mu \mathrm{m}$ drops required $360 \mathrm{~V}$ to be positioned to the corresponding outlets. The second type entailed sorting pairs of droplets having the same size but different contents. Three pairs of droplets: $7.5 \mu m$ silicon oil and carbon grease, $7 \mu m$ silicone oil and carbon-plus- silver grease, and $7.5 \mu \mathrm{m}$ carbon grease and carbon-plus-silver grease were sorted by applying 320 $\mathrm{V}$ for the first pair and $360 \mathrm{~V}$ for the third and second pair respectively. It was necessary to consider the electrical conductivity of the suspending fluid case by case for complete separation. Although, the deionized water worked for the most cases, the third pair of content based sorting required the use of $0.4 \mathrm{mM} \mathrm{K2HPO} 4$ solution instead.

\subsection{Summary of Previous Work}

In the preceding sections we have seen different separation processes based on microscale physics. There are two commonalities that most of the aforementioned separations have in common: the presence of an interface between the species to be separated and the dependency on either external energy inputs or chemical agents. Only 6 passive systems for separation of immiscible fluids have been examined based on the wettability difference between the contiguous phases and the confining surfaces. However, these systems are integrated with an external pressure control to assist the separation process. These controllers may fail due to changes in flow conditions and may add complexity to the separation system.

It is desirable to have truly passive separation processes that do not require any external agents or control. As stated previously, this work explores passive separation of immiscible and miscible fluid mixtures by controlling the interfacial forces 
between fluid components and microchannel surfaces. As a novel approach, the Lattice Boltzmann Method is employed to simulate two-phase immiscible and single phase two component flow and study passive separations without external inputs through fluid-fluid and fluid-surface interactions. In the next chapter, we develop the LBM computational model and describe the fluid-fluid and fluid-solid intermolecular interactions. 


\section{Chapter 3}

\section{Physical and Numerical Modelling}

\subsection{Boltzmann Equation}

In kinetic theory, ensembles of molecules are tracked in order to describe their average behavior by probability distribution functions [43]. This description is the ground for determining the macroscopic properties of fluid systems from the microscopic depiction $[44,45]$. The evolution of distribution functions with time is concurrently related to changes in macroscopic quantities leading to mass, momentum, and energy transfer. To generalize the mentioned transfer phenomena, the Boltzmann transport equation (BTE) emerges as strong tool to establish an alternative approach to satisfy the conservation laws. The principal notion of the (BTE) is explained in the following steps [46]: The number of molecules that travel within the velocity range $\boldsymbol{c}$ to $\boldsymbol{c}+d \boldsymbol{c}$ over a space vector ranging from $\boldsymbol{x}$ to $\boldsymbol{x}+d \boldsymbol{x}$ at the time t reads:

$$
d N=f(\boldsymbol{c}, \boldsymbol{x}, t) d \boldsymbol{x}^{3} d \boldsymbol{c}^{3}
$$

where $f(\boldsymbol{c}, \boldsymbol{x}, t)$ is the probability distribution function.

Similarly, the number of molecules that occupy another element located at $\boldsymbol{x}+\boldsymbol{c} d t$ 
and have a velocity $\boldsymbol{c}+\boldsymbol{a} d t$ after $d t$ is

$$
d N^{\prime}=f(\boldsymbol{c}+\boldsymbol{a} d t, \boldsymbol{x}+\boldsymbol{c} d t, t+d t) d \boldsymbol{x}^{3} d \boldsymbol{c}^{3}
$$

Thus, the net change in the number of colliding molecules becomes

$$
d N_{\text {coll }}=d N^{\prime}-d N=[f(\boldsymbol{c}+\boldsymbol{a} d t, \boldsymbol{x}+\boldsymbol{c} d t, t+d t)-f(\boldsymbol{c}, \boldsymbol{x}, t)] d \boldsymbol{x}^{3} d \boldsymbol{c}^{3}
$$

By taking the limit Eq 3.3 as $d t \rightarrow 0$ yields

$$
\Omega(f)=\frac{\partial f}{\partial \boldsymbol{c}} \boldsymbol{a}+\frac{\partial f}{\partial \boldsymbol{x}} \boldsymbol{c}+\frac{\partial f}{\partial t}
$$

where the operator $\Omega(f)$ accounts for the transition from the initial to the final state of the distribution function. This collision operator controls how the particles interact, determines the path of each population in the post collision step, and sets the number of unknowns relative to its sophisticating $\Omega(f)[47]$.

The fact that collisions become ineffective at equilibrium state implies that $\Omega\left(f_{e q}, f_{e q}\right)=$ 0 which paves the road to linearization of the collision operator presuming that the distribution functions are not far away from the equilibrium [48]. Qain and coworkers [49] noticed from the requirement of viscosity that the collision operator in the Boltzmann Equation could be taken exactly as a relaxation technique in computational fluid dynamics such that

$$
\Omega\left(f, f_{e q}\right)=-\frac{1}{\tau}\left(f-f_{e q}\right)
$$

where $\tau$ is the relaxation time. This approximation is similar to BGK (Bhatangar, Gross, and Krook) model originating from kinitic theory to account for binary colli- 
sions in ionized and neutral gases [50].Thus, the BTE becomes

$$
\frac{\partial f}{\partial t}+\frac{\partial f}{\partial \boldsymbol{c}} \boldsymbol{a}+\frac{\partial f}{\partial \boldsymbol{x}} \boldsymbol{c}=-\frac{1}{\tau}\left(f-f_{e q}\right)
$$

where $f_{e q}$ is the Maxwellian equilibrium distribution function that describes the number of particle having a microscopic velocity $\boldsymbol{c}$ around the local mean velocity $\boldsymbol{u}$. $f_{e q}$ is expressed as

$$
f_{e q}=\frac{\rho}{(2 \pi R T)^{3 / 2}} \exp \left[-\frac{(\boldsymbol{c}-\boldsymbol{u})^{2}}{2 R T}\right]
$$

where $R$ and $\mathrm{T}$ are the universal gas constant and the fluid temperature.

\subsection{Lattice Boltzmann Method}

Starting from the Boltzmann equation with the BGK collision parameter, Eq 3.6, we discretize the velocity space of $\boldsymbol{c}$ into a finite set of velocities $\boldsymbol{c}_{\boldsymbol{i}}$ provided that conservation laws are satisfied in order to maintain the Galilian invariance and to avoid other solutions arising from the broad generality of Boltzmann transport equation (BTE. For the immisicble case we investigate, particles in a three dimensional space confined by 27 velocity vectors. For the miscible mixture case a two dimensional space is confined by 9 velocity vectors. The corresponding lattice weights, $w_{i}$ controls the relative influence of each vector during the streaming process. The discretized form of BTE becomes

$$
\frac{\partial f_{i}}{\partial t}+\frac{\partial f_{i}}{\partial x} c_{i}=-\frac{1}{\tau}\left(f_{i}-f_{i}^{e q}\right)+F_{i}
$$

where $F_{i}$ is the discrete forcing term which includes internal as well as external forces. In addition to the velocity set used in this work shown in Figure 3.1, different sets like D3Q13, D3Q15, and D3Q19 are also possible but not preferred due to either 
the weakness of their stability or the deviation from the Galilean invariance. White and Chong implemented the rotational invariance test to investigate the accuracy of D3Q15, D3Q19, and D3Q27 in axisymmetric flows [51]. They observed that flow features are significantly affected in the directions parallel to planes containing five velocity vectors or less. The observed rotational variance is more apparent with higher flow complexity and asymmetric boundaries. Because all planes in D3Q27 and D2Q9 contain nine velocity vectors, they exhibit higher accuracy than other sets. The truncation error analyses, conducted in [52], showed that D3Q15, D3Q19, and D3Q27 are all able to capture Navier-Stokes hydrodynamics within the continuum borders. However, the Q15 and Q19 lattices exhibit inconsistent physics when discretized due to the non-linear truncation error arising from lack of isotropy. That imposes D3Q27, despite of it's computational overhead, as a robust candidate for stable and accurate LBM simulations. Since D3Q27 is an extension of D2Q9 [53], it's robustness is intrinsically inherited from the D2Q9 lattice; therefore, we adopt the D2Q9 set for studying the miscible mixtures in $2 \mathrm{D}$.

The velocity matrix for the D3q27 lattice is:

$$
\begin{aligned}
{\left[\boldsymbol{c}_{1-14}\right] } & =\left[\begin{array}{rrrrrrrrrrrrrr}
0 & 1 & -1 & 0 & 0 & 0 & 0 & 1 & -1 & -1 & 1 & 1 & -1 & 0 \\
0 & 0 & 0 & 1 & -1 & 0 & 0 & 1 & -1 & 1 & -1 & 0 & 0 & 1 \\
0 & 0 & 0 & 0 & 0 & 1 & -1 & 0 & 0 & 0 & 0 & 1 & -1 & 1
\end{array}\right] \\
{\left[\boldsymbol{c}_{15-27}\right] } & =\left[\begin{array}{rrrrrrrrrrrrrr}
0 \\
0 & -1 & 1 & 0 & 0 & 1 & -1 & 1 & -1 & -1 & 1 & 1 & -1 \\
-1 & 0 & 0 & -1 & 1 & 1 & -1 & 1 & -1 & 1 & -1 & -1 & 1 \\
-1 & 1 & -1 & 1 & -1 & 1 & -1 & 1 & 1 & 1 & -1 & 1 & -1
\end{array}\right]
\end{aligned}
$$

and the weighting coefficients that are carefully selected to preserve the isotropy of 

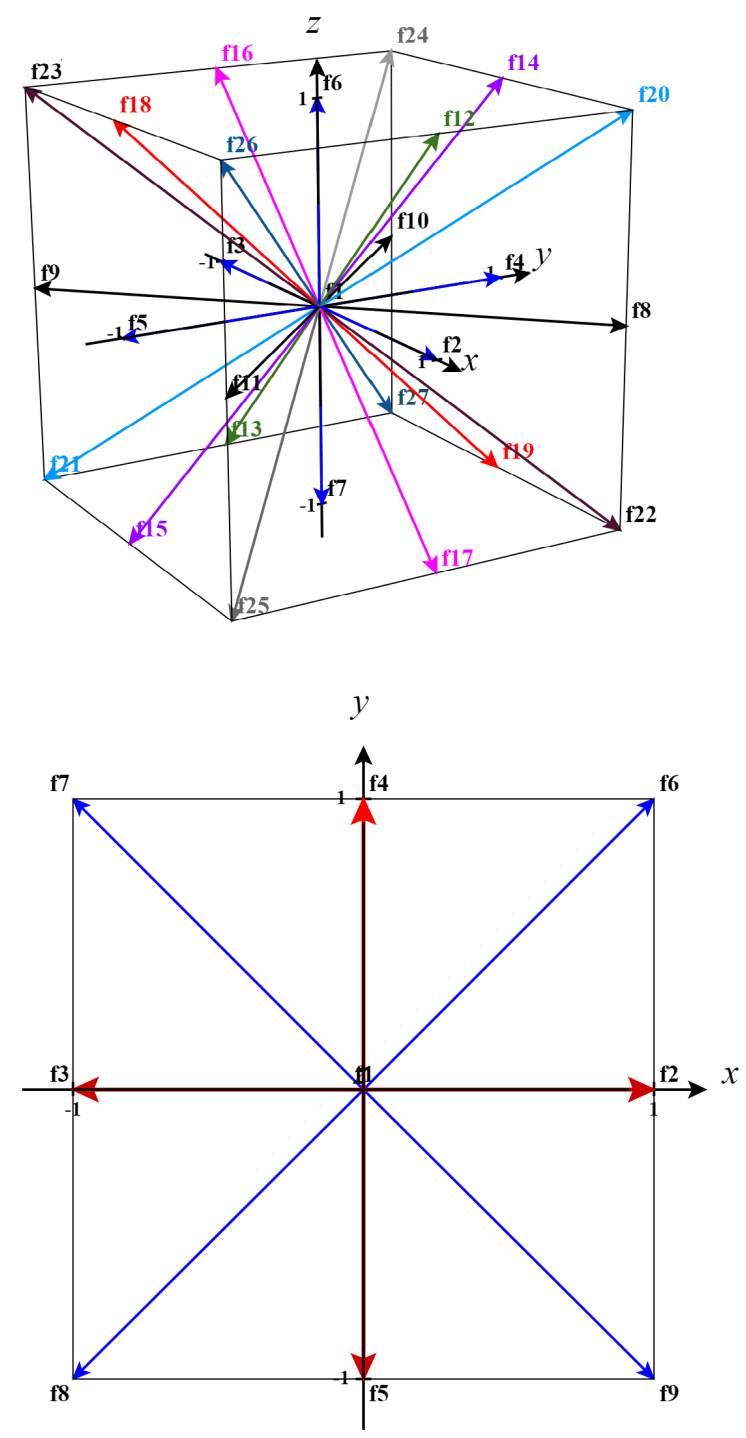

Figure 3.1: The D3q27 [top] and D2q9 [bottom] lattice sets used in this work for immiscible and miscible cases respectively. 
the velocity tensor and maintain the Galilian invariance [49] are

$$
\left[\boldsymbol{w}_{\boldsymbol{i}}\right]=\left\{\begin{array}{ccc}
\frac{8}{27} & \text { for } & i=1 \\
\frac{2}{27} & \text { for } & i=2-7 \\
\frac{1}{54} & \text { for } & i=8-19 \\
\frac{1}{216} & \text { for } & i=20-27
\end{array}\right.
$$

while the velocity matrix for the D2Q9 lattice is

$$
\left[c_{i}\right]=\left[\begin{array}{ccccccccc}
0 & 1 & -1 & 0 & 0 & 1 & -1 & -1 & 1 \\
0 & 0 & 0 & 1 & -1 & 1 & 1 & -1 & -1
\end{array}\right]
$$

and the lattice weights are

$$
\left[\boldsymbol{w}_{\boldsymbol{i}}\right]=\left\{\begin{array}{ccc}
\frac{4}{9} & \text { for } & i=1 \\
\frac{1}{9} & \text { for } & i=2-5 \\
\frac{1}{36} & \text { for } & i=6-9
\end{array}\right.
$$

The Gauss Hermite quadrature rule indicates that the discretized equilibrium distribution function, $f_{e q}$, appears as

$$
f_{i}^{e q}=w_{i} \rho\left(1+\frac{\boldsymbol{c}_{\boldsymbol{i}} \cdot \boldsymbol{u}}{c_{s}^{2}}+\frac{\left(\boldsymbol{c}_{\boldsymbol{i}} \cdot \boldsymbol{u}\right)^{2}}{2 c_{s}^{4}}-\frac{\boldsymbol{u} \cdot \boldsymbol{u}}{2 c_{s}^{2}}\right)
$$

$c_{s}$ refers to the lattice speed of sound which is usually taken as $1 / \sqrt{3}$ to maintain numerical stability[54]. Additionally, the moments of the equilibrium as well as the particle distribution function are used to calculate the local density, momentum, and 
energy as

$$
\begin{aligned}
\rho & =\sum_{i=1}^{q} f_{i}=\sum_{i=1}^{q} f_{i}^{e q} \\
\boldsymbol{u} & =\frac{1}{\rho} \sum_{i=1}^{q} f_{i} \boldsymbol{c}_{\boldsymbol{i}}=\frac{1}{\rho} \sum_{i=1}^{q} f_{i}^{e q} \boldsymbol{c}_{\boldsymbol{i}} \\
E & =\frac{1}{2 \rho} \sum_{i=1}^{q} f_{i}\left|\boldsymbol{c}_{\boldsymbol{i}}\right|^{2}=\frac{1}{2 \rho} \sum_{i=1}^{q} f_{i}^{e q}\left|\boldsymbol{c}_{\boldsymbol{i}}\right|^{2}
\end{aligned}
$$

In order to implement the LBE for computational purposes, we still need additional discretization in space and time domains. This is achieved by the method of characteristics. Hence, we have the trajectory $\zeta$ such that $x=x(\zeta)$ and $t=t(\zeta)$ which implies

$$
\frac{d f_{i}}{d \zeta}=\frac{\partial f_{i}}{\partial t} \frac{d t}{d \zeta}+\frac{\partial f_{i}}{\partial x} \frac{d x}{d \zeta}
$$

Integrating the LBE from $\zeta=0$ to $\zeta=\Delta t$ yields:

$$
f_{i}\left(\boldsymbol{x}+\boldsymbol{c}_{\boldsymbol{i}} \Delta t, t+\Delta t\right)=f_{i}(\boldsymbol{x}, t)-\frac{\Delta t}{\tau}\left[f_{i}(\boldsymbol{x}, t)-f_{i}^{e q}(\boldsymbol{x}, t)\right]+\Delta t S_{i}
$$

where $S_{i}$ is discretised forcing term which appears in different forms depending on the construction schemes. Here we follow the Guo forcing

$$
S_{i}=\left(1-\frac{\Delta t}{2 \tau}\right) w_{i}\left(\frac{\boldsymbol{c}_{\boldsymbol{i}}-\boldsymbol{u}}{c_{s}^{2}}+\frac{\left(\boldsymbol{c}_{\boldsymbol{i}} \cdot \boldsymbol{u}\right) \boldsymbol{c}_{\boldsymbol{i}}}{c_{s}^{4}}\right) \cdot \boldsymbol{F}
$$

This guarantees the second order accuracy in velocity and space-time discretization

\subsection{Streaming and Collision}

The underlying procedures of the LBM consist of two alternating steps: collision and streaming. The collision step is the process where the populations coming from the adjacent nodes through the lattice links relax to their equilibrium state following the collision rules accounting, in a deterministic manner, for conservation of mass and 
momentum [55]. Momentum is exchanged among the participating particles and their directions of motion are reoriented[56]. The populations resulting from the collision process represented by the right hand side of $\mathrm{Eq}(3.16)$ are

$$
f_{i}^{*}(\boldsymbol{x}, t)=f_{i}(\boldsymbol{x}, t)-\frac{1}{\tau}\left[f_{i}(\boldsymbol{x}, t)-f_{i}^{e q}(\boldsymbol{x}, t)\right]
$$

The streaming step is the process that propagates the post collision populations, $f_{i}^{*}$, to the neighboring nodes through the corresponding links. In this situation, each node receives its updated distribution functions from their surroundings and send the opposite of what it has received, schematically shown in Figure 3.2.

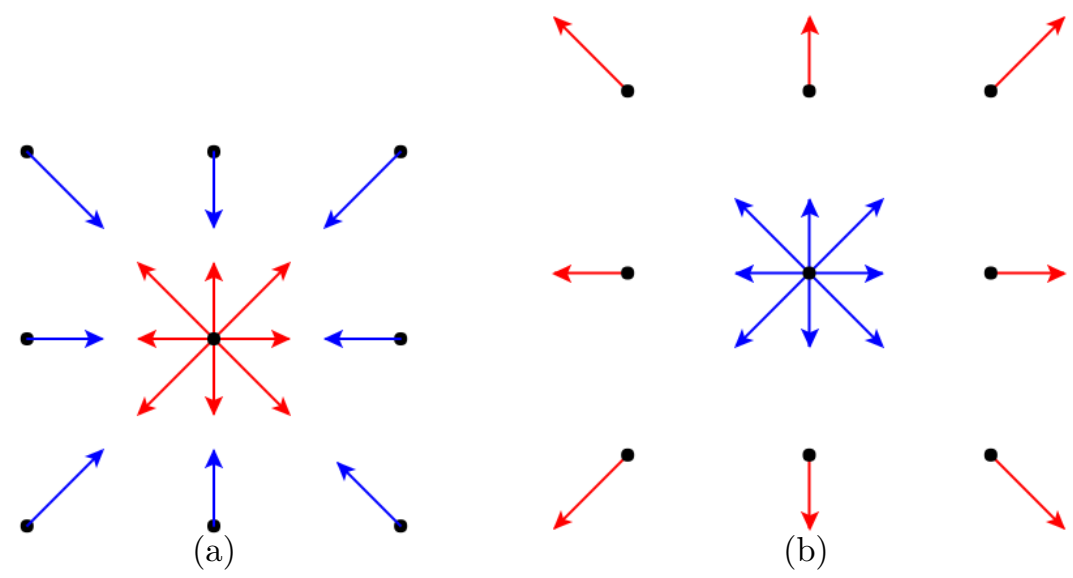

Figure 3.2: Streaming process of a D2Q9 velocity set (a) the pre-streaming condition (b) the post streaming condition.

The propagation step is equivalent to the left hand side of $\operatorname{Eq}(3.16)$

$$
f_{i}\left(\boldsymbol{x}+\boldsymbol{c}_{i} \Delta t, t+\Delta t\right)=f_{i}^{*}(\boldsymbol{x}, t)
$$

It is imperative to select the time and space steps in such a way that all the populations arrive at the next adjacent nodes after the streaming process without being entangled between nodes. As a result, the LBM community normally applies the 
unitary temporal and spacial steps: $\Delta t=\Delta x_{\alpha}=1$.

\subsection{Boundary Conditions}

LBM boundary conditions follow a different approach compared with the traditional CFD techniques like finite difference, finite element, or finite volume method. The straightforwardness of imposing Dirichlet or Neumann conditions in these techniques has to be replaced with multidegree of freedom depending on the velocity set used in LBM.

In the present work we encounter four cases of macroscopic boundary conditions; 1) the no slip velocity at solid walls, 2) the moving walls implementation at the inlet, 3) the convective outflow applied to side channels outlets, and 4) the periodic boundary conditions applied to the inlet/exit of the 2-D miscible binary mixture.

The zero velocity condition at the confining walls is prescribed using the Bounce Back (BB) method which states fluid particles impinge a solid objects and rebound in the reverse direction back to the fluid domain. Instead of locating the fluid boundary nodes exactly on the wall, BB places the computational boundary at distance $\Delta x / 2$ from the real boundary which guarantees a second order boundary conditions accuracy due to the involvement of second order spacial discretization [57]. The sequence of implementation is started by streaming the post collision populations pointing out of the computational domain at time $t$ from the the first layer of the fluid nodes to the adjacent solid wall nodes. After that, the populations stored in the solid nodes are replaced with their opposites and streamed again after $\Delta t$ in order to update the unknown populations, to those pointing to the inside. Figure 3.3 demonstrates this. 


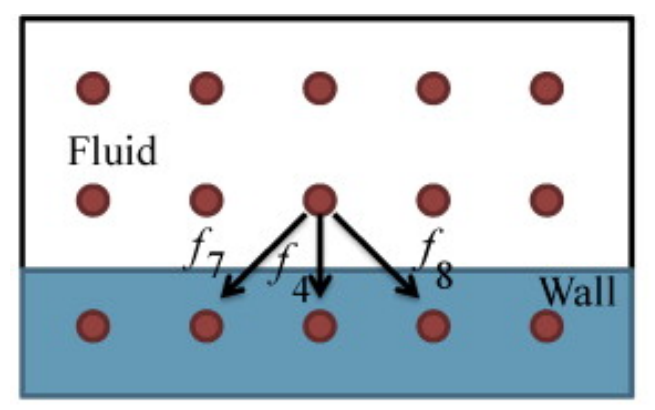

(A) before streaming

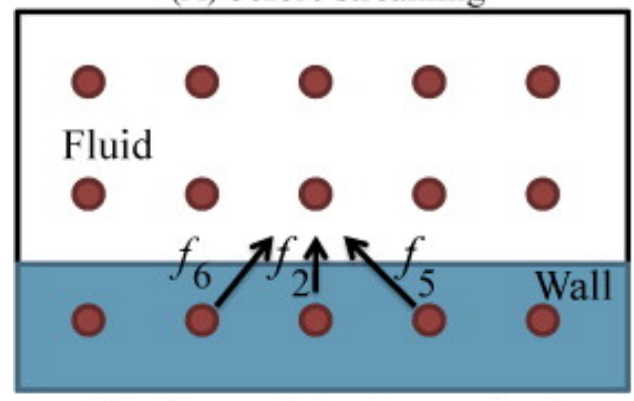

(C) after applying bounce-back

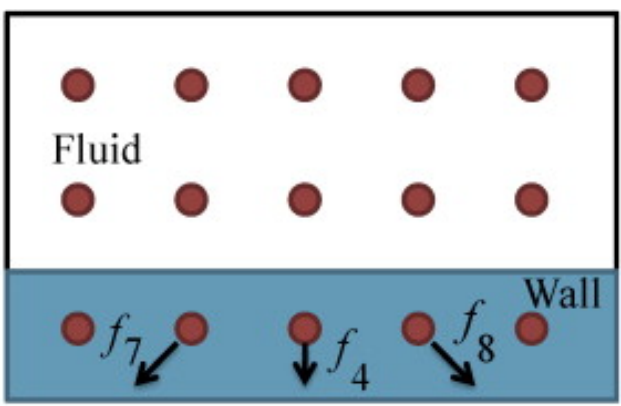

(B) after streaming

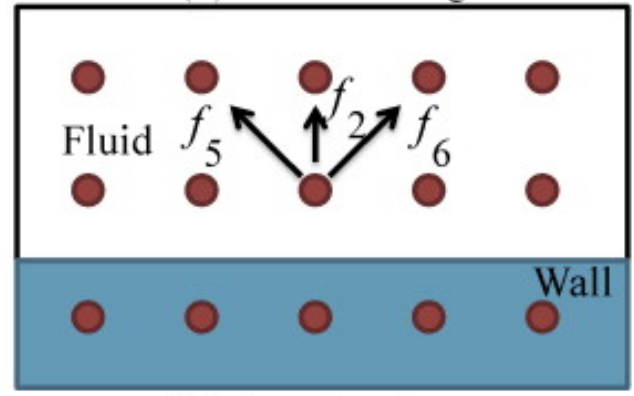

(D) streaming stage

Figure 3.3: The Bounce Back (BB) process used to impose the no slip boundary conditions [57].

Computationally this means

$$
f_{i}\left(\boldsymbol{x}_{\boldsymbol{b}}, t+\Delta t\right)=f_{\bar{i}}^{*}\left(\boldsymbol{x}_{\boldsymbol{b}}, t\right)
$$

where $x_{\boldsymbol{b}}$ refers to the fluid boundary point and $\bar{i}$ indicated the opposite direction.

For the immiscible case, the moving boundary bounce back [58-60] is applied to 
the inlet boundary conditions of the T-channel such that

$$
\begin{aligned}
f_{6} & =f_{7}-2 w_{7} \rho_{i n} \frac{\boldsymbol{e}_{\mathbf{7}} \cdot \boldsymbol{u}_{i n}}{c_{s}^{2}} \\
f_{12} & =f_{13}-2 w_{13} \rho_{i n} \frac{\boldsymbol{e}_{13} \cdot \boldsymbol{u}_{i n}}{c_{s}^{2}} \\
f_{14} & =f_{15}-2 w_{15} \rho_{i n} \frac{\boldsymbol{e}_{15} \cdot \boldsymbol{u}_{i n}}{c_{s}^{2}} \\
f_{16} & =f_{17}-2 w_{17} \rho_{i n} \frac{\boldsymbol{e}_{17} \cdot \boldsymbol{u}_{i n}}{c_{s}^{2}} \\
f_{18} & =f_{19}-2 w_{19} \rho_{i n} \frac{\boldsymbol{e}_{19} \cdot \boldsymbol{u}_{i n}}{c_{s}^{2}} \\
f_{20} & =f_{21}-2 w_{21} \rho_{i n} \frac{\boldsymbol{e}_{21} \cdot \boldsymbol{u}_{i n}}{c_{s}^{2}} \\
f_{23} & =f_{22}-2 w_{22} \rho_{i n} \frac{\boldsymbol{e}_{22} \cdot \boldsymbol{u}_{i n}}{c_{s}^{2}} \\
f_{24} & =f_{25}-2 w_{25} \rho_{i n} \frac{\boldsymbol{e}_{25} \cdot \boldsymbol{u}_{i n}}{c_{s}^{2}} \\
f_{26} & =f_{27}-2 w_{27} \rho_{i n} \frac{\boldsymbol{e}_{27} \cdot \boldsymbol{u}_{i n}}{c_{s}^{2}}
\end{aligned}
$$

where $\rho_{\text {in }}$ and $\boldsymbol{u}_{\text {in }}$ are the inlet density and flow velocity respectively. It is worth mentioning that the dispersed phase density is set to zero at the inlet to prevent irregular droplets formation due to pressure jumps when the original drop passes the the bifurcation point. The result is Eq 3.21 becomes Eq 3.20 for the discrete phase only.

The outflow boundary conditions for multiphase flows are not as straightforward as those in single phase flows. The difficulty arises from the advection and wave propagation dominating the transport process which require a special treatment to open boundaries in order to avoid distortion or disturbance reflections to the interior domain [61]. In [62], it was shown that the convective boundary condition, Neumann boundary condition, and the extrapolation boundary conditions exhibit variant attitudes when used for two-phase flows. It was emphasized that convective out flow 
condition is the best choice for such cases; therefore, we are going to adopt it for the outlets of the T-junction in immiscible fluids separation. It is defined as

$$
\frac{\partial \phi}{\partial t}+U \frac{\partial \phi}{\partial x}=0
$$

where $\phi$ is any variable to be updated at the boundary nodes. In our case $\phi$ stands for the force components, the density, and the distribution function. $U$ is taken as the flow velocity normal to the outlet and it has three possibilities: local, average, or maximum. In our case, the last option was the best as it lead to more reasonable physics of the two-phase flow. For the T-junction outlets, $\mathrm{U}$ is expressed as

$$
\begin{array}{r}
U_{\max }(1, y, z, t)=\max \{u(2, y, z, t)\} \\
U_{\max }(N, y, z, t)=\max \{u(N-1, y, z, t)\}
\end{array}
$$

Equation 3.22 is discretized as [62]

$$
\begin{aligned}
\frac{\partial \phi}{\partial t} & =\frac{\phi(x, y, z, t)-\phi(x, y, z, t-\delta t)}{\delta t} \\
\frac{\partial \phi}{\partial x} & =\frac{\phi(x, y, z, t)-\phi(x-\delta x, y, z, t)}{\delta x}
\end{aligned}
$$

The resulting discrete form becomes

$$
\phi(x, y, z, t)=\frac{\phi(x, y, z, t-\delta t)+U_{\max }(t) \phi(x-\delta x, y, z, t)}{1+U_{\max }(t)}
$$

The last category of boundary conditions is the periodic implementation used for the miscible case. It is assumed there is a virtual layer between the inlet and outlet of the channel. This entails streaming the post collision populations leaving the outlet to update their inlet unknown counterparts. The same procedure applies to those 
leaving the inlet where they substitute for the outlet unknowns, prescribed as

$$
\begin{array}{lll}
f_{6}(1, y, t)=f_{6}\left(N_{x}, y, t\right) & f_{2}(1, y, t)=f_{2}\left(N_{x}, y, t\right) & f_{9}(1, y, t)=f_{9}\left(N_{x}, y, t\right) \\
f_{7}\left(N_{x}, y, t\right)=f_{7}(1, y, t) & f_{3}\left(N_{x}, y, t\right)=f_{3}(1, y, t) & f_{8}\left(N_{x}, y, t\right)=f_{8}(1, y, t)
\end{array}
$$

\subsection{Shan Chen Model for Multiphase Multicomponent Flows}

This model of LBM approach was first introduced by Shan and Chen $[63,64]$ to simulate flows including multicomponents, multiphases, or single component undergoing phase change. The main purpose of the model is to overcome the artifacts arising in the first generation of LBM models represented by the non-Galilean invariant nature and the nonphysical interfacial characteristics when simulating components of different species. For this purpose, a non-local interaction potential among populations is incorporated in the computation of individual velocities of each component. This potential is

$$
\varphi^{i}\left(\boldsymbol{x}, \boldsymbol{x}+\boldsymbol{c}_{\boldsymbol{i}} \Delta t\right)=G_{\sigma \tilde{\sigma}} \psi^{\sigma}(\boldsymbol{x}) \psi^{\tilde{\sigma}}\left(\boldsymbol{x}+\boldsymbol{c}_{\boldsymbol{i}} \Delta t\right)
$$

where $\psi^{\sigma}$ is the density function or the effective mass of the individual component $\sigma$,

which is a function of the local density $\psi=\rho_{0}\left(1-e^{\frac{-\rho}{\rho_{0}}}\right)$. The justification for choosing this function instead of the density itself is to suppress any numerical instability that may arise due to high density ratios [43]. The interaction function, $G_{\sigma \tilde{\sigma}}$ is a Green's function and it is active only within the closest site as

$$
G_{\sigma \tilde{\sigma}}^{i}(\Delta \boldsymbol{x})=\left\{\begin{array}{cc}
0 & \text { if }|\Delta x|>c_{i} \Delta t \\
g_{\sigma \tilde{\sigma}} & \text { if }|\Delta x|=c_{i} \Delta t
\end{array}\right.
$$


The parameter $g_{\sigma \tilde{\sigma}}$ is the substratum of this model due to its hegemonic power to adjudicate whether the interaction between $\sigma \tilde{\sigma}$ is repulsive or attractive. Consequently, the affinity among components is controlled such that miscibility or immiscibility is decided from this foundational stone. It is considered as a free parameter that determine the ratio of potential to thermal energy [65].

The total intermolecular force, $\boldsymbol{F}^{\sigma}(\boldsymbol{x})$, locally acting on the center node of the component $\sigma$ is simply evaluated by the accumulative effect of the non-local potentials acting though all the lattice links

$$
\boldsymbol{F}^{\sigma}(\boldsymbol{x})=-\psi^{\sigma}(\boldsymbol{x}) \sum_{\tilde{\sigma}=1}^{S} g_{\sigma \tilde{\sigma}} \sum_{i=1}^{Q} w_{i} \psi^{\tilde{\sigma}}\left(\boldsymbol{x}+\boldsymbol{c}_{\boldsymbol{i}} \Delta t\right) \cdot \boldsymbol{c}_{\boldsymbol{i}} \Delta t
$$

where $\mathrm{S}$ is the number of fluids components and $\mathrm{Q}$ is the total number of lattice vectors, which is 27 in the two-phase flow case and 9 in the binary miscible mixture case. According to Newton's second law, this force impacts a change in momentum. Therefore, the new equilibrium velocity should be updated as

$$
\boldsymbol{u}^{e q, \sigma}(\boldsymbol{x})=\frac{1}{\rho^{\sigma}(\boldsymbol{x})}\left[\sum_{i=1}^{Q} f_{i}^{\sigma} \boldsymbol{c}_{\boldsymbol{i}}+\tau \boldsymbol{F}^{\sigma}(\boldsymbol{x})\right]
$$

Accordingly, the overall flow velocity of the multi-compnent system which is equivalent to the velocity obtained from the solution of Navier Stokes equation is expressed as

$$
\boldsymbol{u}=\frac{1}{\rho} \sum_{\sigma}\left[\sum_{i} f_{i}^{\sigma} \boldsymbol{c}_{\boldsymbol{i}}+\frac{\Delta t \boldsymbol{F}^{\sigma}}{2}\right]
$$

where $\rho=\sum_{\sigma} \rho^{\sigma}$ is the overall local density of the system. To illustrate the set up of the Shan-Chen model in our problem, Figure 3.4 exhibits three categories of nodes based on the reciprocal interactions between them and their neighbors. The first group is the interior nodes (colored blue) where a fluid at the lattice center interacts 
with other fluids at the the peripheral nodes of that lattice. The second group is the computational boundary of the fluid (black nodes) where each fluid component is affected by both the wall material and other fluid components. The third group is the solid wall nodes (colored red), which interact with both fluid components through the lattice links pointing toward the bulk domain.

Mathematically, the aforementioned classifications of nodes have to be handled separately. The core nodes are treated using the general Shan-Chen force model, using Eq 3.29, except for those located at the inlet and outlet where the classic boundary conditions apply. The nodes located adjacent to the walls are subject to a special case of eq 3.29 , which is

$$
\boldsymbol{F}^{\sigma}(\boldsymbol{x})=-\psi^{\sigma}(\boldsymbol{x})\left[G_{\sigma \tilde{\sigma}} \sum_{\text {blue }} \psi^{\tilde{\sigma}}\left(\boldsymbol{x}+\boldsymbol{c}_{\boldsymbol{i}} \Delta t\right) \boldsymbol{c}_{\boldsymbol{i}}+G_{\sigma S} \sum_{\text {red }} \psi^{S}\left(\boldsymbol{x}+\boldsymbol{c}_{\boldsymbol{i}} \Delta t\right) \boldsymbol{c}_{\boldsymbol{i}}\right] \Delta t
$$

\subsection{Multirange Pseudopotential for Binary Mixture}

As any approximation scheme, LBM has limitations and shortcomings. Particularly, the Shan-Chen model has two main weaknesses: spurious currents resulting from force discretization and the thermodynamic inconsistency due to the connection between density ratio and surface tension [43]. Including additional lattice cells beyond the first belt was one of the remedies to enhance the stability of the interaction forces due to the contribution of soft-core interactions and hence attenuating the intense local interactions [66]. Multiple belts are constructed around the local node to increase the order of isotropy [65] (see Figure 3.5). 

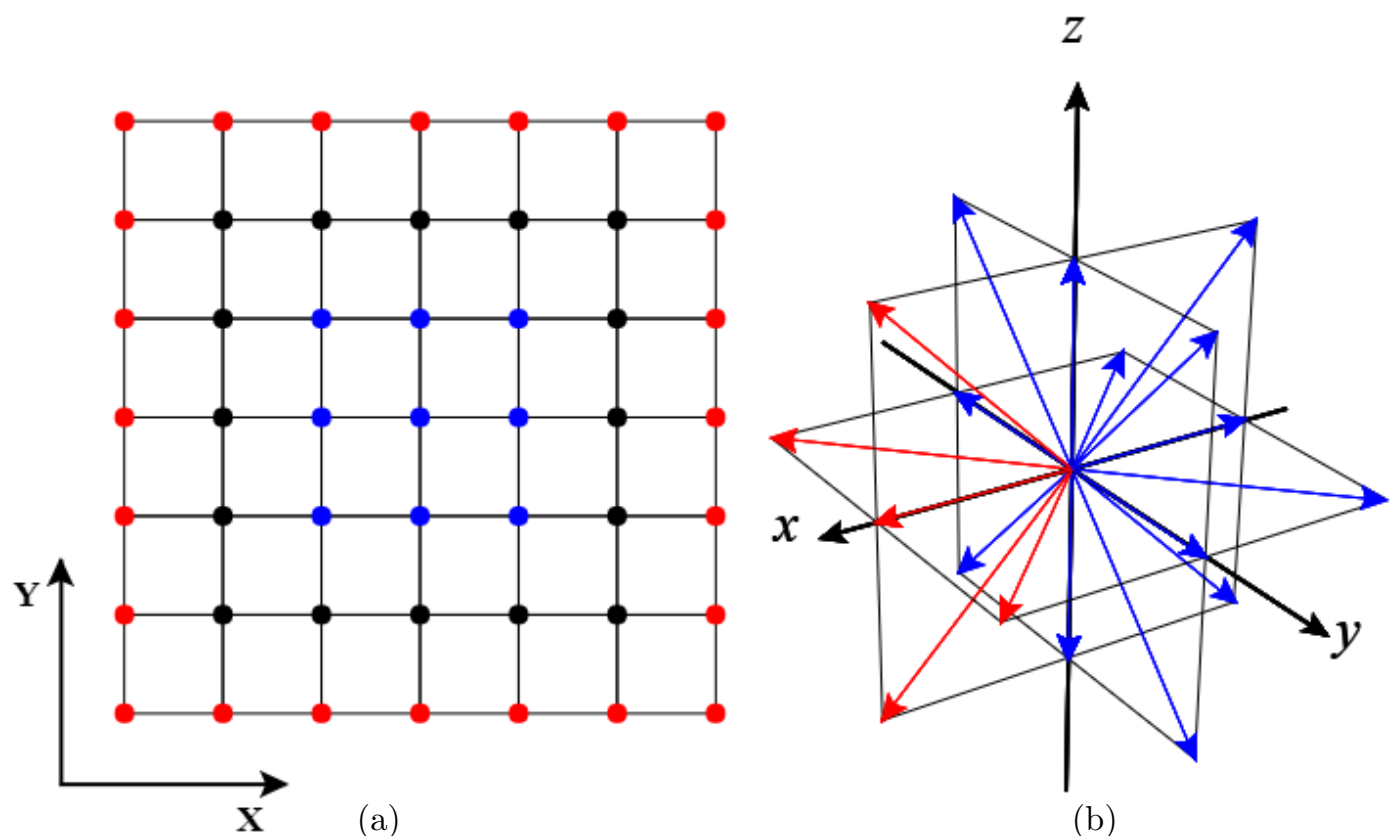

(a)

(b)

Figure 3.4: A fluid node adjacent to a solid surface interacting with another fluid through blue vectors and the solid nodes through the red links.

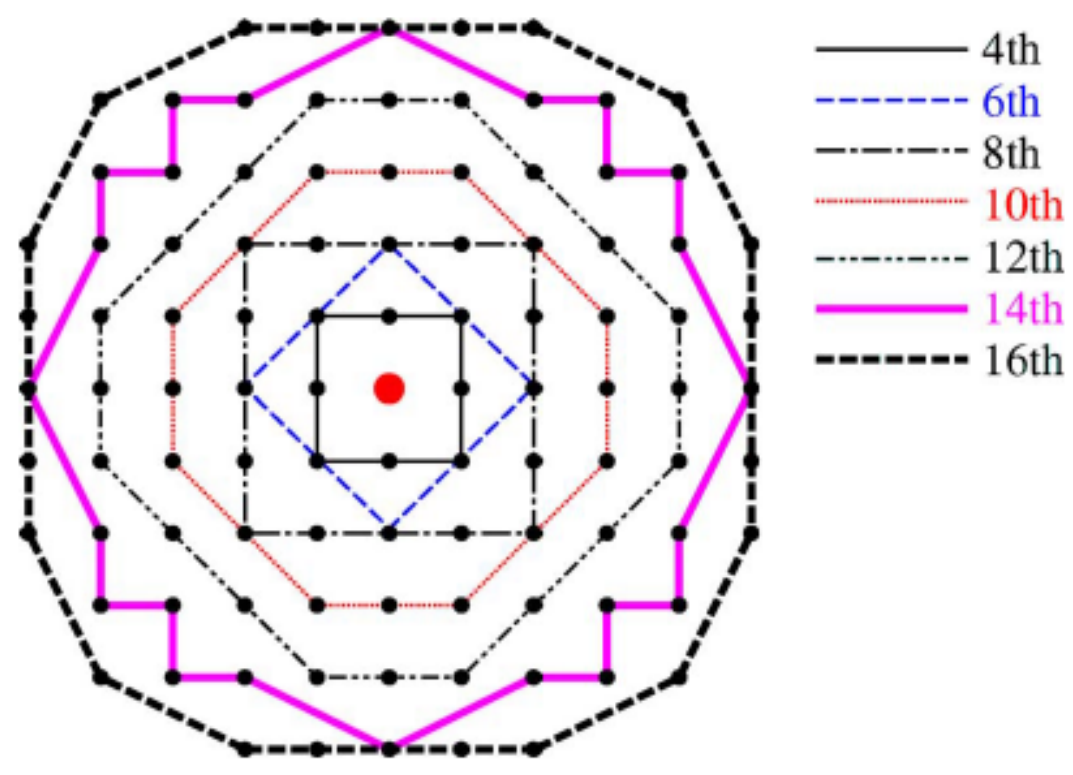

(a)

Figure 3.5: The multirange lattice with the order of isotropy identifying the extra nodes of the interaction forces [65]. 
Thus, the interaction force formula we mentioned in Eq 3.29 is modified to

$$
\boldsymbol{F}^{\sigma}(\boldsymbol{x})=-\psi^{\sigma}(\boldsymbol{x}) \sum_{\tilde{\sigma}=1}^{S} g_{\sigma \tilde{\sigma}} \sum_{i=1}^{Q} w\left(\left|\boldsymbol{c}_{\boldsymbol{i}}\right|^{2}\right) \psi^{\tilde{\sigma}}\left(\boldsymbol{x}+\boldsymbol{c}_{\boldsymbol{i}} \Delta t\right) . \boldsymbol{c}_{\boldsymbol{i}} \Delta t
$$

The main change here is that the lattice velocities $\boldsymbol{c}_{\boldsymbol{i}}$ are extended to the external neighbors and new lattice weights $w\left(\left|\boldsymbol{c}_{\boldsymbol{i}}\right|^{2}\right)$, corresponding to the new force links, are applied. Sbragaglia et al [65] derived these weights based on the isotropy order of interaction forces for both $2 \mathrm{~d}$ and $3 \mathrm{~d}$ cases. Here, we are motivated by the extension of surface forces effect to the furthest point for the binary mixture flowing in $2 \mathrm{~d}$ microchannels while keeping the standard lattice for the two-phase flow in $3 \mathrm{~d}$ microchannel; therefore, we need only the new weights up to the 6 th belt in a $2 \mathrm{D}$ lattice. Table 3.1 provides these weights.

It is important to keep in mind that the remaining hydrodynamics such as collisions, forces in 3D, velocities, and densities are still using the same lattice weights we referred earlier in the Equations 3.10 and 3.12 . 
Table 3.1: The extended force weights up to the 6th belt in $2 \mathrm{D}[65]$.

\begin{tabular}{ll|ll}
\hline$\left|\boldsymbol{c}_{\boldsymbol{i}}\right|^{2}$ & Lattice weight $w\left(\left|\boldsymbol{c}_{\boldsymbol{i}}\right|^{2}\right)$ & $\left|\boldsymbol{c}_{\boldsymbol{i}}\right|^{2}$ & Lattice weight $w\left(\left|\boldsymbol{c}_{\boldsymbol{i}}\right|^{2}\right)$ \\
\hline 1 & $\frac{285860656}{3979934595}$ & 17 & $\frac{231568}{3979934595}$ \\
2 & $\frac{2113732952}{43779280545}$ & 18 & $\frac{1516472}{43779280545}$ \\
4 & $\frac{940787801}{43779280545}$ & 20 & $\frac{18769}{1591973838}$ \\
5 & $\frac{124525000}{8755856109}$ & 255,0 & $\frac{184}{315867825}$ \\
8 & $\frac{15841927}{3979934595}$ & 253,4 & $\frac{464}{795986919}$ \\
9 & $\frac{2046152}{795986919}$ & 26 & $\frac{1448}{4864364505}$ \\
10 & $\frac{14436304}{8755856109}$ & 29 & $\frac{148}{4864364505}$ \\
13 & $\frac{18185828}{43779280545}$ & 32 & $\frac{629}{400267707840}$ \\
16 & $\frac{13537939}{140093697744}$ & & \\
\hline
\end{tabular}

\subsection{Physical Mapping}

Real world applications involve quantities with physical units. Whether those quantities are fundamental (mass, length, time) or derived (force, acceleration, viscosity...etc), they have to be mentioned for the sake of lucid interpretation. Subsequently, LBM results, which are expressed in simulation units, must be converted to real units.

Our top priority is to maintain the Galilean invariance by proper mapping from physical domain to simulation domain and vice versa. Thus, the fundamental conversions are realized first by matching the temporal and spacial conversions between the two domains. The length and time mapping are based on $\Delta x^{p}[m]$ and $\Delta t^{p}[\mathrm{sec}]$ respectively.

The selection of $\Delta x^{p}[m]$ and $\Delta t^{p}[\mathrm{sec}]$ is not random as they are coupled to each other. This coupling depends on the system under consideration with different coupling for binary mixtures and multiphase flows. In case of a single phase fluid com- 
posed two chemical species of different properties, each component moves with a different speed and hence different $\Delta x^{p}$ for a fixed $\Delta t^{p}[67]$. Therefore, the barycentric properties of the mixture are used instead of the individual properties to link $\Delta x^{p}$ with $\Delta t^{p}$. The mixture speed of sound, $c_{s-m i x}$, and kinematic viscosity, $\nu_{m i x}$ are employed as

$$
\begin{array}{r}
d t^{p}=\frac{\nu_{\text {mix }}}{c_{s-m i x}^{2}\left(\tau_{m i x}-0.5\right)} \\
d x^{p}=\sqrt{3} d t^{p} \cdot c_{s-m i x}
\end{array}
$$

In the case of two-phase flow, the spatial steps of both fluids are already unified because the two-phases are coupled by the interface. Accordingly, the distance moved by particles of each fluid are the same. In addition to the coupling in Eq 3.34, which is called acoustic scaling $\Delta t^{p} \propto \Delta x^{p}$, there is another approach to connect the temporal and spacial steps by the diffusive scaling $\Delta t^{p} \propto \Delta x^{p^{2}}[43,68]$. If we pick $\Delta x^{p}=1.0 \times 10^{-6}[\mathrm{~m}]$, the time step is $10^{-6}[\mathrm{sec}]$ for the acoustic scaling and $10^{-12}[\mathrm{sec}]$ for the diffusive approach. However, both approaches make it difficult to match the surface tension to a realistic value. In other words, the mapped surface tension is either higher or lower than the measured values of common fluids. Therefore, we found that $\Delta t^{p} \propto \Delta x^{p 1.25}$, following the more general linking $\Delta t^{p} \propto \Delta x^{p \alpha}$ where $\alpha \geq 1$ [43], gives a reasonable mapping of surface tension and the viscosities of both components.

We know from the streaming and collision section that $\Delta t=\Delta x_{\alpha}=1$. As simulation variables they are dimensionless with the time and length conversion factors appearing as 


$$
\begin{gathered}
C_{t}=\frac{\Delta t^{p}}{\Delta t}=\Delta t^{p} \\
C_{l}=\frac{\Delta x^{p}}{\Delta x}=\Delta x^{p}
\end{gathered}
$$

The remaining fundamental conversion is drawn from the density such that

$$
C_{\rho}=\frac{\rho^{p}}{\rho^{L B}}
$$

Thus, the mapping factors for velocity, viscosity, mass, surface tension, and pressure gradient are

$$
C_{u}=\frac{C_{l}}{C_{t}}, \quad C_{\nu}=\frac{C_{l}^{2}}{C_{t}}, \quad C_{m}=C_{\rho} \cdot C_{l}^{3}, \quad C_{\sigma}=\frac{C_{m}}{C_{t}^{2}}, \quad C_{\nabla p}=\frac{C_{\rho} \cdot C_{l}}{C_{t}^{2}}
$$

\subsection{Contact Angle Measurements}

The contact angle, $\theta_{c}$, is the most common method to evaluate fluid-surface interactions due to its simplicity [15]. Referring to Young's equation [69]:

$$
\gamma_{S G}=\gamma_{S L}+\gamma_{L G} \cos \left(\theta_{c}\right)
$$

where $\gamma_{S G}, \gamma_{S L}, \gamma_{L G}$ are the interfacial energies of solid-gas, solid-liquid, and liquidgas regions respectively, it is evident that surface wettability is mainly controlled by the manipulation of the solid-liquid interfacial energy, $\gamma_{S L}$, or the liquid-gas counterpart, $\gamma_{L G}[12]$, as shown in the Figure 3.6 [70]. 


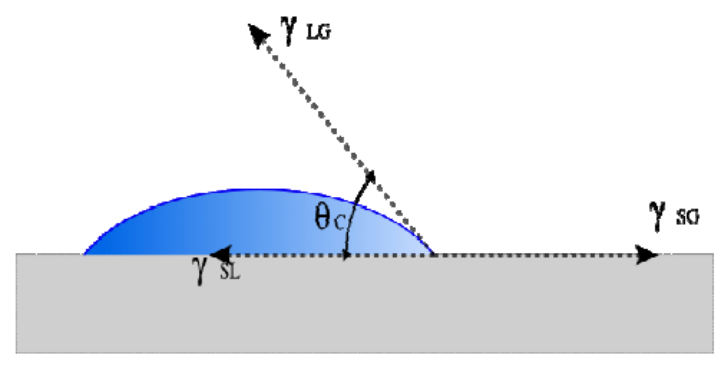

Figure 3.6: Static balance of interfacial tensions of a liquid drop on a solid surface

The control of the liquid-solid surface tension is the most ubiquitous and tangible tactic to achieve the appropriate/coveted wetting conditions. This can be accomplished by different stratagems such as thermo-wetting where the $\gamma_{S L}$ depends on the local temperature of the solid-liquid interface [71-74], and electro-wetting which involves an application of voltage between the droplet and its substrate to modify the value of $\gamma_{S L}[75-77]$.

For two immiscible liquids, $\gamma_{L G}$, is replaced by $\gamma_{L L}[15,16,78,79] . \gamma_{L L}$ is modified to control the wettability under both static and dynamic conditions. Its influence on contact line topographies originates from the difference in the free energy due to the formation of the interface.

In order to understand the effects of solid-fluid interaction coefficients, the resulting contact angles due to the competition between these coefficients were measured using ImageJ software [80]. The final density profiles were obtained by placing a square cube of fluid-1 $\left(\rho_{1}=1.0\right)$ inside a cavity filled with fluid-2 $\left(\rho_{2}=0.77\right)$. The height of the initialized cube is 20 lattice cells while the base area is $40 \times 40$ square cells, which was attached to the bottom wall of the container. The relaxation times were taken $\tau_{1}=0.548$, and $\tau_{2}=1.0$ for fluid- 1 and fluid- 2 respectively. The cross interaction coefficient and the self interaction of fluid- 1 were fixed at $G_{1-2}=6.0$ and 
$G_{1-1}=-0.75$ respectively while the solid-fluid interactions ranged from 0.0 to 3.0. The simulation was run for $\frac{(W / 2)^{2}}{\left(\tau_{1}-0.5\right) / 3}$ time steps to ensure that $\rho_{1}$ reached the steady state profile. A series of contact angles, measured from inside the drop using the Contact angle plugin of ImageJ software (as seen in Figure (3.7), were produced. The contact angles are summarized in Table (3.2). The corresponding density profiles are shown in Figure (3.8).

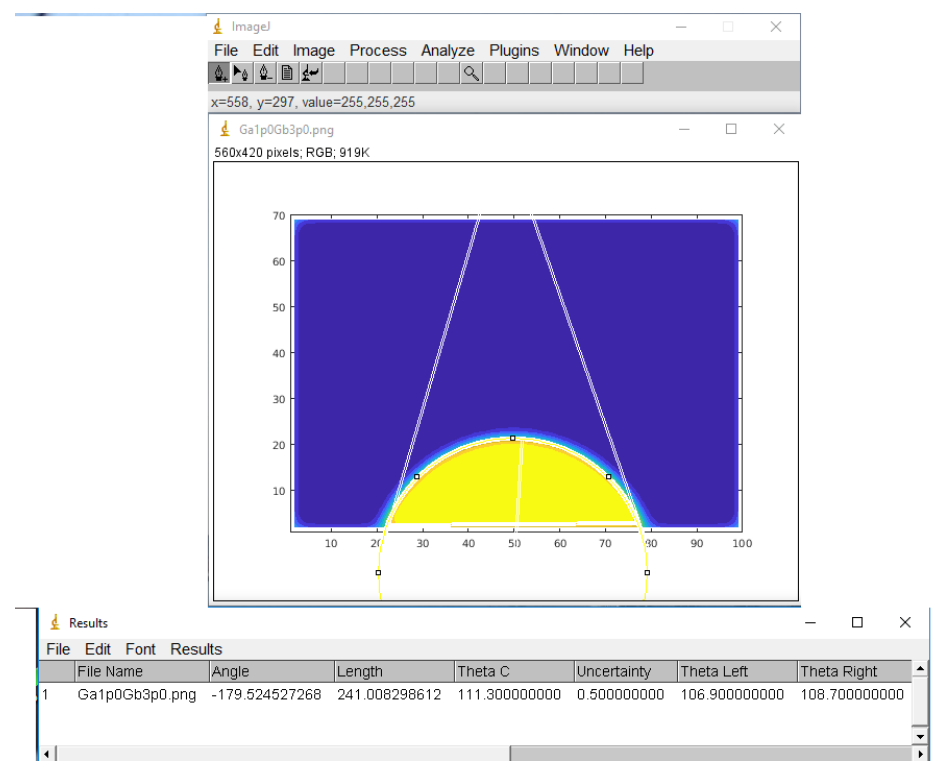

Figure 3.7: A snapshot of contact angle measurement using ImageJ software. The result Theta $\mathrm{C}$ is the quantity of interest. We subtract it from $180^{\circ}$ to calculate the contact angle from inside.

Table 3.2: Resulting Contact Angles due to Solid-Fluid interaction parameters variations.

\begin{tabular}{|c|c|c|c|c|c|c|c|}
\hline Case & $G_{1 s}$ & $G_{2 s}$ & Contact angle & Case & $G_{1 s}$ & $G_{2 s}$ & Contact angle \\
\hline A & 0.0 & 3.0 & $43.0 \pm 0.2$ & $\mathrm{H}$ & 3.0 & 2.5 & $103.2 \pm 0.6$ \\
\hline B & 0.5 & 3.0 & $57.7 \pm 0.3$ & $\mathrm{I}$ & 3.0 & 2.0 & $110.4 \pm 0.7$ \\
\hline $\mathrm{C}$ & 1.0 & 3.0 & $69.1 \pm 0.4$ & $\mathrm{~J}$ & 3.0 & 1.5 & $120.4 \pm 0.7$ \\
\hline $\mathrm{D}$ & 1.5 & 3.0 & $76.8 \pm 0.5$ & $\mathrm{~K}$ & 3.0 & 1.0 & $131.4 \pm 0.3$ \\
\hline $\mathrm{E}$ & 2.0 & 3.0 & $83.2 \pm 0.3$ & $\mathrm{~L}$ & 3.0 & 0.5 & $143.2 \pm 0.8$ \\
\hline $\mathrm{F}$ & 2.5 & 3.0 & $90.3 \pm 0.7$ & $\mathrm{M}$ & 3.0 & 0.0 & $173.2 \pm 7.1$ \\
\hline $\mathrm{G}$ & 3.0 & 3.0 & $95.2 \pm 0.4$ & & & & \\
\hline
\end{tabular}




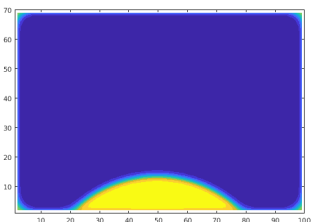

(a)

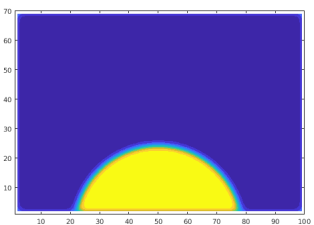

(d)

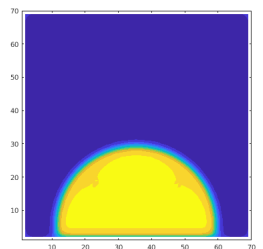

(g)

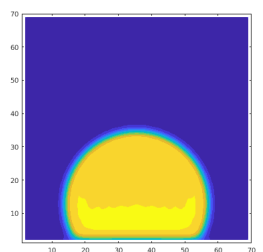

(j)

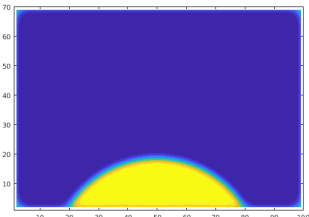

(b)

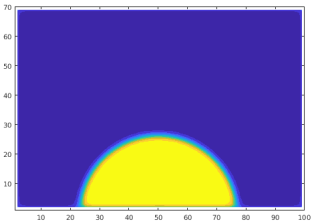

(e)

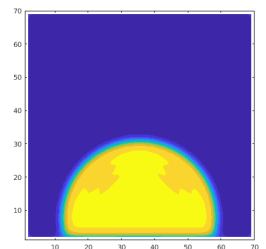

(h)

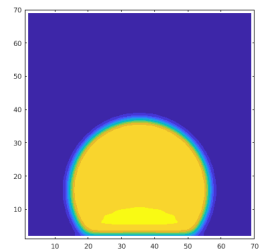

$(\mathrm{k})$

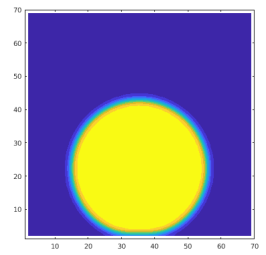

(m)

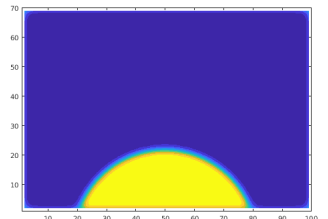

(c)

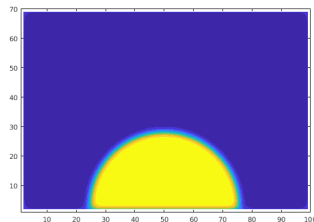

(f)

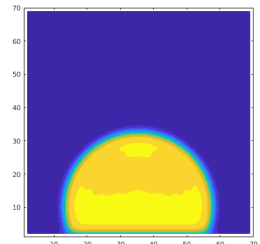

(i)

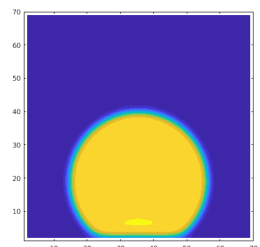

(l)

Figure 3.8: Density profiles of two immiscible fluids at $G_{12}=6.0, G_{1-1}=-0.75$ and different surface interactions coefficients. Fluid densities are $\rho_{1}=1.0$, and $\rho_{2}=0.77$.

\subsection{Young-Laplace Test}

The Young-Laplace test was implemented to ensure that in two-phase flows, the pressure difference between inside and outside of a simulated drop increases linearly 
with the inverse of drop radius:

$$
\Delta p=\frac{2 \sigma}{R}
$$

where $\sigma$ is the surface tension, and $\mathrm{R}$ is the radius of the drop. To conduct this test, we initialized 3-D spherical drops of fluid-1 $\left(\rho_{1}=1.0, \tau_{1}=0.548\right)$ inside a cavity filled with fluid-2 $\left(\rho_{2}=0.77, \tau_{2}=1.0\right)$. Due to the diffusive nature of the interface, it is normal to encounter different densities than was initialized. Therefore, minor densities of each fluid are prescribed before starting the simulation. These densities represent the dissolved portion of one phase in the other phase. The simulation is stopped after $\frac{(W / 2)^{2}}{\left(\tau_{1}-0.5\right) / 3}$ iterations where both densities and the size of droplet reach their steady profiles. The radius and pressure drop are measured at steady state. Due to the isotropy issues in Shan-Chen model, the inner pressure is not necessarily homogeneous. Thus, we take 5 points in all directions surrounding the center and average their pressures to achieve better conformance of surface tension. Table 3.3 provides the $\Delta P$ for each radius and the calculated $\sigma$. Figure 3.9 shows the linear curve fitting of $2.0 / R$ with $\Delta P$ indicates that the surface tension is $\sigma=0.044$.

Table 3.3: Numerical results of Young-Laplace test for spherical drops of fluid-1 surrounded by fluid-2 at $G_{12}=6.0, G_{11}=-0.75$.

\begin{tabular}{|c|c|c|c|c|c|}
\hline $\mathrm{R}$ & 12.150 & 15.550 & 18.657 & 21.756 & 24.707 \\
\hline$\Delta P$ & 0.0074 & 0.0058 & 0.0048 & 0.0041 & 0.0038 \\
\hline$\sigma$ & 0.0448 & 0.0450 & 0.0450 & 0.0449 & 0.0450 \\
\hline
\end{tabular}




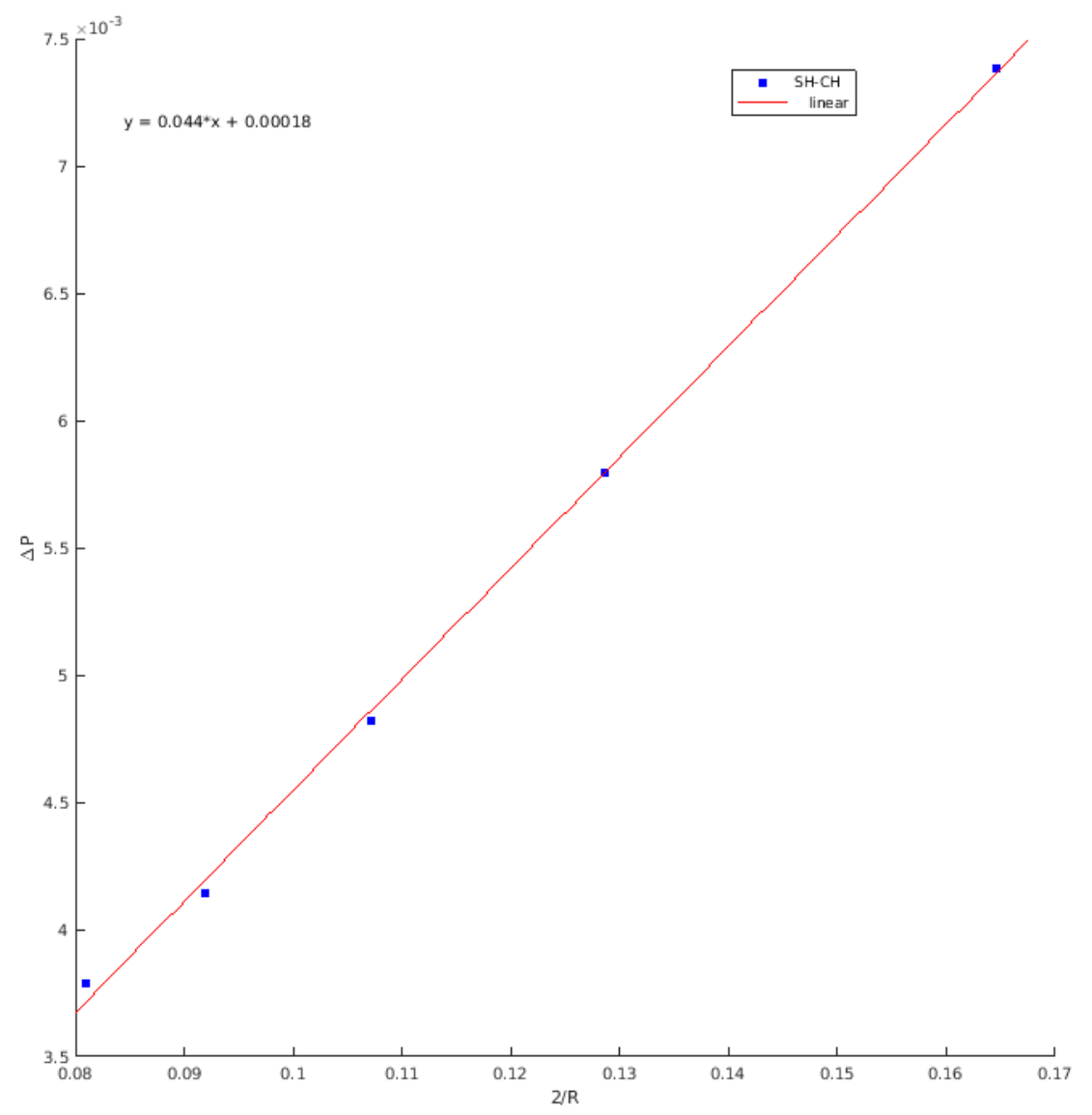

Figure 3.9: Linear fitting of pressure difference vs $2 / \mathrm{R}$

\subsection{Numerical Validation}

To test the validity of the computational approach and developed set of equations such that the resulting data is treated as physically consistent, the LBM code is applied to a well known analytic solution, two dimensional Poiseuille flow, and a published computational study of drop breakup in a T-junction channel.

\subsection{Two dimensional Poiseuille Flow}

The analytic solution of pressure driven fluid flow between two parallel plates is well known in the literature and textbooks of fluid mechanics. The velocity profile of flow 
in a channel of height $\mathrm{W}$ with the no slip boundary conditions is defined as [81]

$$
u(y)=-\frac{d p}{d z} \frac{W^{2}}{8 \mu}\left(1-\frac{4 y^{2}}{W^{2}}\right)
$$

where $\frac{d p}{d z}$ is the pressure gradient and $\mu$ is the dynamic viscosity. It is straightforward to implement Eq 3.40 to check the validity of the velocity profile calculated with the multi-range multi-components LBM with a couple of caveats. First, we know Eq 3.40 is an analytic solution of the Naveir-Stokes equation which is valid for a single fluid. Therefore, it is imperative to simulate two species of the same fluid properties using the LBM model. Because LBM allows the existence of both species locally, a mixing rule based on mass fraction is required. Thus, the mass fraction of a species is

$$
X_{\sigma}=\frac{\rho_{\sigma}}{\sum_{\sigma} \rho_{\sigma}}
$$

The overall viscosity of the system, which appears in Eq 3.40 is

$$
\mu=X_{1} \mu_{1}+X_{2} \mu_{2}
$$

The pressure gradient $\frac{d p}{d z}$ is simply taken as the forcing term, $F_{x}$, in the X-direction.

Second, the interaction forces between the two fluid components needs to be described. This demand is realized by making the cross and self interaction the same sign and magnitude, attractive in our case. This yields single fluid behavior from a model which is essentially intended for multicomponents mixtures.

Figure 3.10 shows the comparison between the exact solution and the D2Q9 multirange pseudo-potential model for two fluid species. A good agreement is observed with maximum relative error less than $17 \%$. 


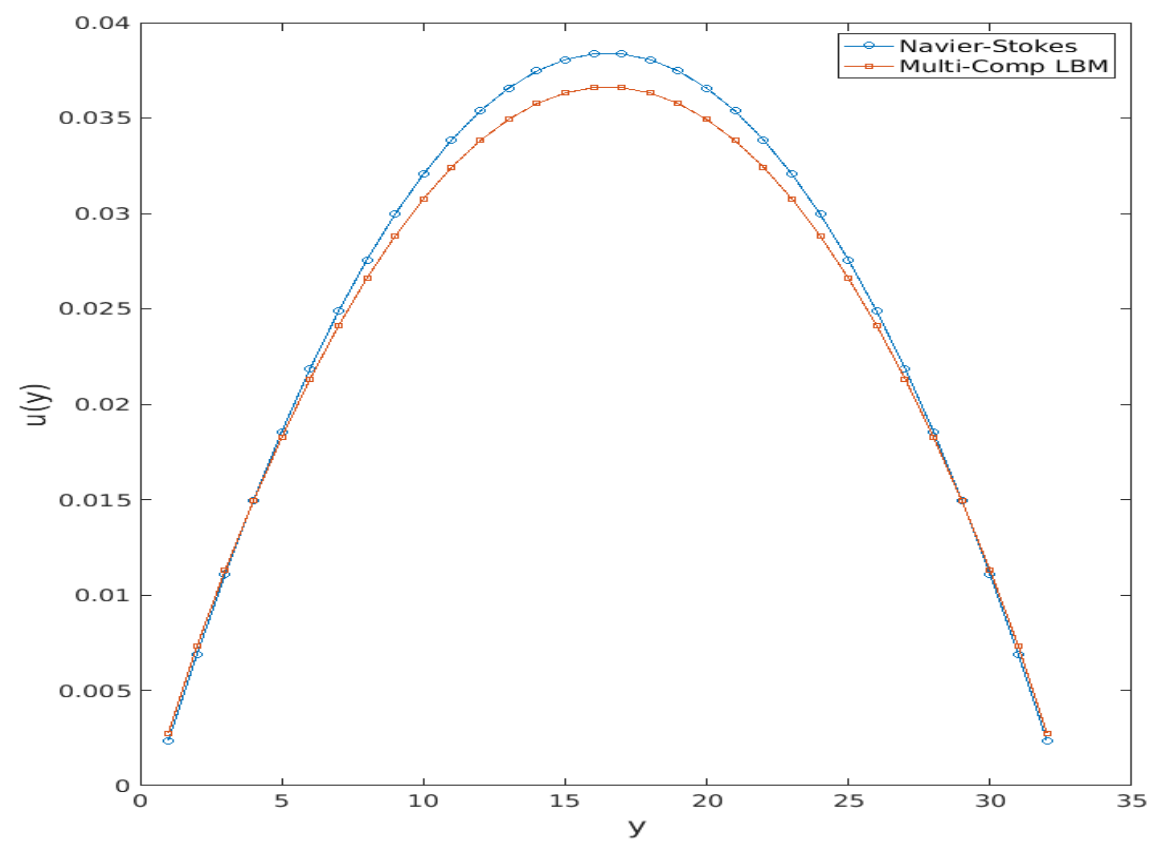

(a)

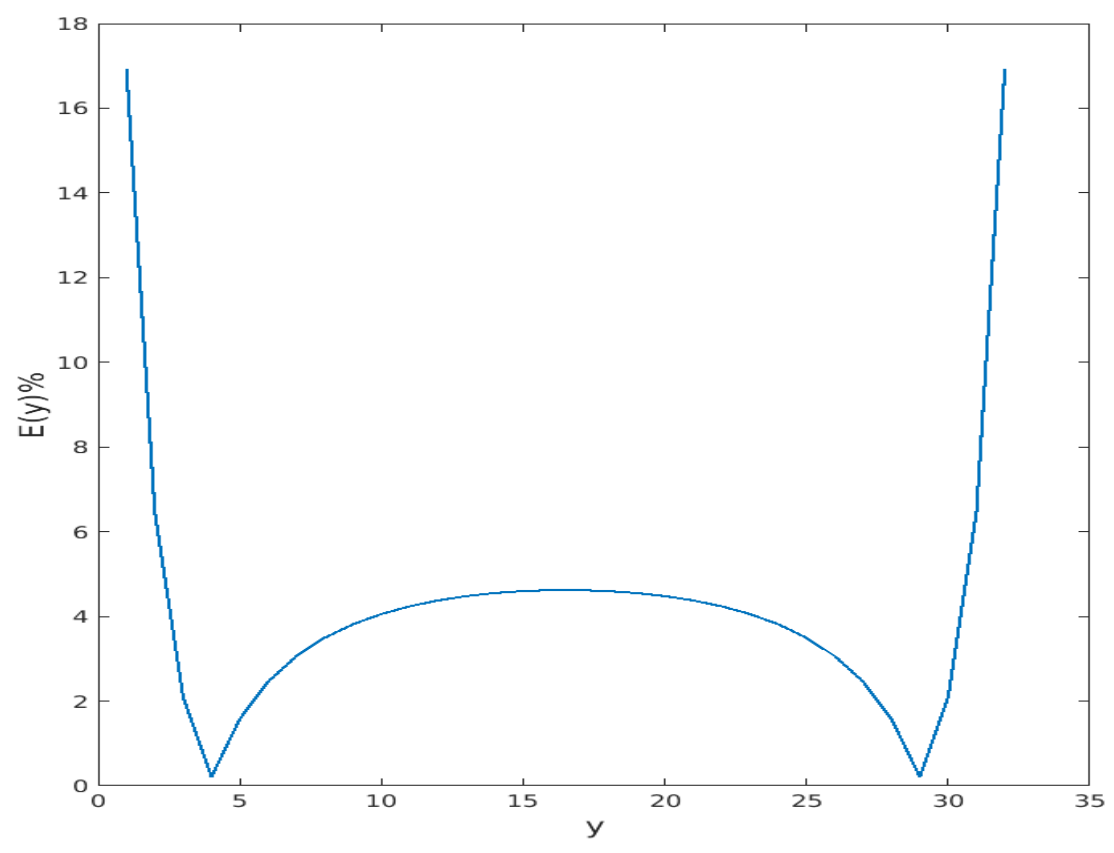

(b)

Figure 3.10: (a)Velocity profile from both N-S exact solution and LBM binary mixture Shan-Chen model when $\frac{d p}{d x}=0.00001, \nu_{1}=\nu_{2}=0.017, \rho_{1}=\rho_{2}=1.0, W=32$ lattices. (b) Relative local error between velocities in (a). The maximum relative error is less than $17 \%$. 
The numerical error shown in Figure $3.10 \mathrm{~b}$ is the absolute value of the local difference between the analytic and the LBM velocity divided by the analytic velocity at the same point.

\subsection{Drop Breakup in a T-Junction}

Applying even wetting conditions to a T-channel, the LBM two-phase flow problem resembles the drop breakup scenario of Hoang et al [82]; with the volume of fluid method (VOF) they studied the breakup of a drop in a T-junction. The drop is driven to the T-channel bifurcation by the continuous phase which is fed to the channel with an inlet average velocity $u_{i n}$. When the droplet leaves the main channel completely, it starts to be squeezed by the continuous fluid as the pressure in the main channel starts to increase. Depending on the length of the confined drop and the capillary number, the neck of the drop shrinks with time until breakup. The evolution rate of the neck is mainly controlled by the competition between surface and viscous forces when fluid inertia is insignificant. Figure 3.11 shows this evolution. As in Hoang et al [82], the capillary number $C a=0.0029,0.0063$, and 0.0078 and the dynamic viscosity ratio is fixed at $\lambda=\frac{1}{8}$. Here, the capillary number is defined as

$$
C a=\frac{\mu_{2} u_{i n}}{\sigma}
$$

where $\mu_{1}$ and $\sigma$ are the dynamic viscosity of the continuous phase and the surface tension obtained from the Laplace-Young test (see Figure 3.9)

The viscosity ratio is defined as

$$
\lambda=\frac{\rho_{1}\left(\tau_{1}-0.5\right)}{\rho_{2}\left(\tau_{2}-0.5\right)}
$$



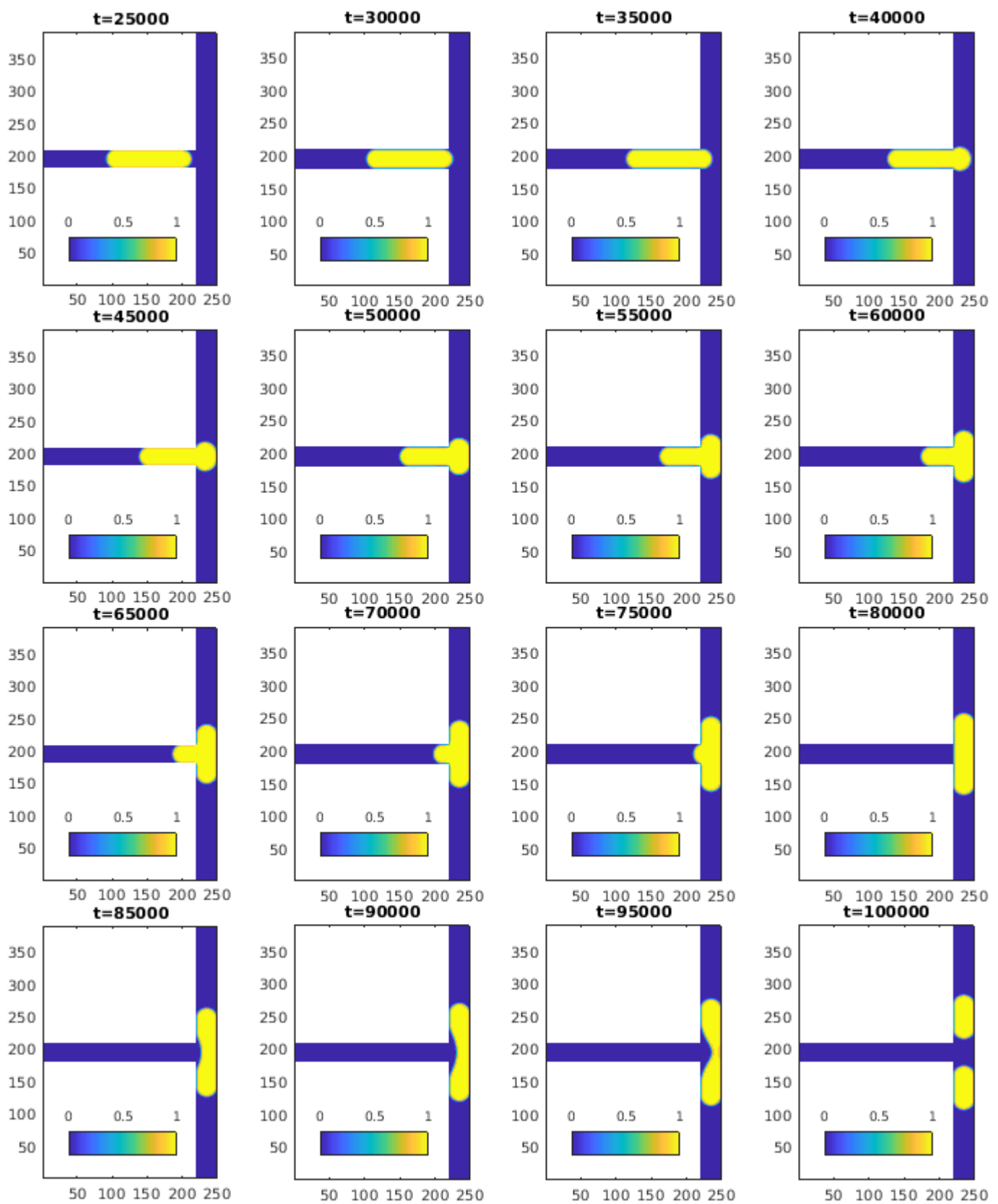

Figure 3.11: The evolution of neck thickness of a droplet of density $\rho_{1}=1.0$ and viscosity $\nu_{1}=0.33$ surrounded by a fluid of density $\rho_{2}=0.77$ and viscosity $\nu_{2}=0.33$ under even wetting conditions $\theta_{\text {upper }}=\theta_{\text {lower }}=173.2 \pm 7.1$ at capillary number $C a=0.0063$ corresponding to Reynolds number $R e=0.381$. The initial droplet length is $L_{0}=3 W$ while the final length $4 \mathrm{~W}$. 
The dimensionless neck width, $\delta^{*}$, is realized by normalizing the neck width, $\delta$, by the channel width, W. Similarly, we use the characteristic time, $t=W / u_{i n}$, to calculate the dimensionless time $t^{*}$.

It is worth mentioning that the comparison data was extracted from Figure 6 (a) in [82] with the aid of WebPlotDigitizer [83].

Figure 3.12 compares the neck width evolution calculated with our LBM simulations and Hoang et al [82] VOF computations. Good agreement is observed for all capillary numbers.

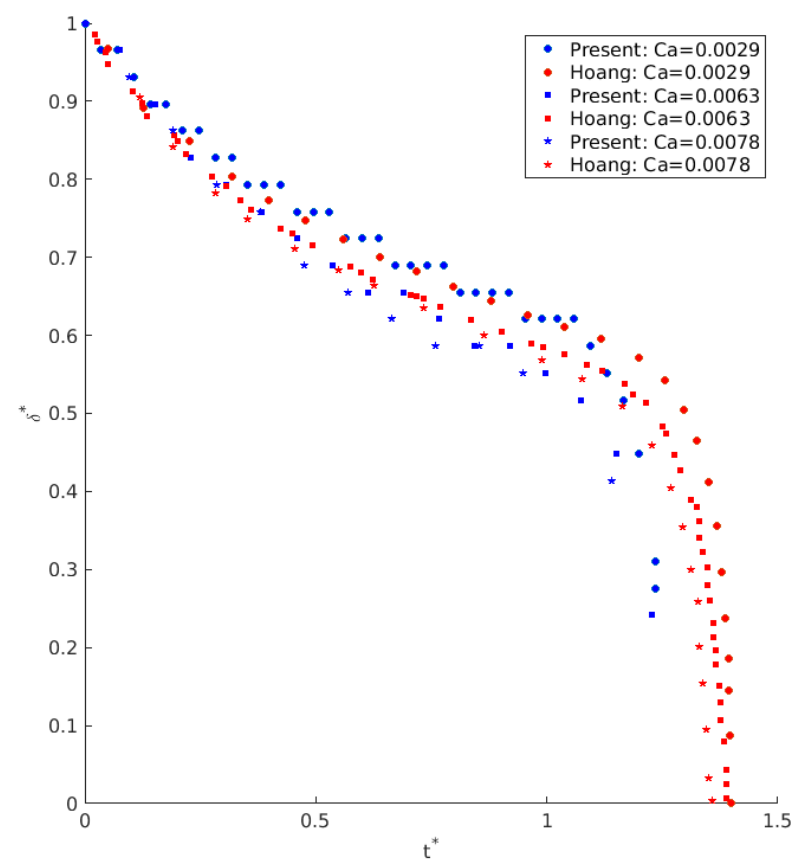

Figure 3.12: Neck width evolution with time: a comparison between present work and the literature. 


\section{Chapter 4}

\section{Miscible Binary Mixture in a Straight Channel}

In this chapter we discuss the results of confining two miscible fluids in a microchannel with variable surface forces. The LBM multirange pseudopotential is implemented to cover a wide range of forces that arise in intermolecular interactions. The number of belts contributing in the Shan-Chen force model are limited to 6. Properties of fluids are chosen to mimic a water/ethanol mixture at $25^{\circ} \mathrm{C}$. The kinematic viscosity and the speed of sound of each component are used to link simulation units to the real values, (see Eq 3.34). The numerical values, listed in Table 4.1, are obtained from Dortmund Data Bank website [84]. The resulting mass fractions are $X_{1}=0.56$ and $X_{2}=0.44$ which are used to calculate the mixture properties in the same manner we calculate the dynamic viscosity in Eq 3.41. The viscosity and sonic speed of the mixture are $1.11 \times 10^{-06}\left[\mathrm{~m}^{2} / \mathrm{s}\right]$ and $1342.8[\mathrm{~m} / \mathrm{s}]$ respectively.

Table 4.1: Physical properties of the fluids used in simulation.

\begin{tabular}{|c|c|c|c|c|c|}
\hline$\rho_{1}\left[\mathrm{Kg} / \mathrm{m}^{3}\right]$ & $\rho_{2}\left[\mathrm{Kg} / \mathrm{m}^{3}\right]$ & $\nu_{1}\left[\mathrm{~m}^{2} / \mathrm{s}\right]$ & $\nu_{2}\left[\mathrm{~m}^{2} / \mathrm{s}\right]$ & $c_{s}^{1}[\mathrm{~m} / \mathrm{s}]$ & $c_{s}^{2}[\mathrm{~m} / \mathrm{s}]$ \\
\hline 1000 & 784 & $8.941 \times 10^{-7}$ & $1.386 \times 10^{-6}$ & 1500 & 1142.29 \\
\hline
\end{tabular}


Eq 3.34 indicates that $\Delta x^{p}$ and $\Delta t^{p}$ are controlled by the mixture relaxation time $\tau_{m}$ which depends on the relaxation times $\tau_{1}$ and $\tau_{2}$ of both fluids. Thus, a range of spatial steps, and corresponding temporal steps, arise based on the chosen relaxation times. Table 4.2 provides the spatial step size as a function of relaxation times. Time steps are obtained by dividing the spatial steps by $\sqrt{3}$ times the mixture speed of sound. Thus, the temporal step scales as a picosecond [ps]. The spacial step size $\Delta x^{p}$ ranges from 2.86 to 28.64 [nm] which is still within the span of the intermolecular forces when 6 belts of lattices are involved in the interaction. In this chapter, the relaxation times are fixed at $\tau_{1}=\tau_{2}=0.75$ unless otherwise stated. The corresponding space and time steps are $\Delta x^{p}=5.72[\mathrm{~nm}]$ and $\Delta t^{p}=4.26[\mathrm{ps}]$ respectively.

In the next sections, the effects of the simulation parameters like cross interaction strength, solid-fluid interaction coefficients, relaxation times, the number of cells between the confining surfaces are studied under static conditions and the densities of both fluids are presented under these effects. Additionally, solid posts with similar wettability to that of the channel half they are are located in are added to enhance the separation of the binary mixture.

Table 4.2: The spacial step size $\Delta x^{p}$ in [nm] as a function of relaxation times $\tau_{1}$ and $\tau_{2}$

\begin{tabular}{|c|l|l|l|l|l|l|l|l|l|l|l|}
\cline { 2 - 13 } \multicolumn{2}{c|}{} & \multicolumn{10}{c|}{$\tau_{1}$} \\
\cline { 2 - 13 } & 0.55 & 0.6 & 0.65 & 0.7 & 0.75 & 0.8 & 0.85 & 0.9 & 0.95 & 1.0 \\
\hline \multirow{4}{*}{$\tau_{2}$} & 0.55 & 28.64 & 19.89 & 15.24 & 12.35 & 10.38 & 8.95 & 7.87 & 7.02 & 6.34 & 5.78 \\
\cline { 2 - 13 } & 0.60 & 18.35 & 14.32 & 11.74 & 9.94 & 8.63 & 7.62 & 6.823 & 6.177 & 5.642 & 5.192 \\
\cline { 2 - 12 } & 0.65 & 13.50 & 11.18 & 9.54 & 8.32 & 7.38 & 6.63 & 6.02 & 5.51 & 5.08 & 4.71 \\
\cline { 2 - 12 } & 0.70 & 10.68 & 9.17 & 8.04 & 7.16 & 6.45 & 5.87 & 5.38 & 4.97 & 4.62 & 4.31 \\
\cline { 2 - 12 } & 0.75 & 8.83 & 7.77 & 6.95 & 6.28 & 5.72 & 5.26 & 4.87 & 4.53 & 4.23 & 3.97 \\
\cline { 2 - 11 } & 0.80 & 7.53 & 6.75 & 6.11 & 5.59 & 5.15 & 4.77 & 4.44 & 4.16 & 3.91 & 3.69 \\
\cline { 2 - 11 } & 0.85 & 6.56 & 5.96 & 5.46 & 5.04 & 4.67 & 4.36 & 4.09 & 3.85 & 3.63 & 3.44 \\
\cline { 2 - 11 } & 0.90 & 5.81 & 5.34 & 4.93 & 4.58 & 4.28 & 4.02 & 3.78 & 3.58 & 3.39 & 3.22 \\
\cline { 2 - 11 } & 0.95 & 5.22 & 4.83 & 4.50 & 4.21 & 3.95 & 3.72 & 3.52 & 3.34 & 3.18 & 3.03 \\
\cline { 2 - 11 } & 1.0 & 4.73 & 4.41 & 4.13 & 3.88 & 3.67 & 3.47 & 3.29 & 3.14 & 2.99 & 2.86 \\
\hline
\end{tabular}




\subsection{Cross Interaction Effect}

To understand the impact of fluid-fluid interaction coefficient $G_{12}$ on the distribution of both components densities, a microchannel of height 30 lattices $(0.17 \mu m)$ and 450 lattices $(2.574 \mu m)$ long is considered. Simulation fluid densities $\rho_{1}=1.0$ and $\rho_{2}=$ 0.784 are initialized in the fluid domain. Periodic boundary conditions are prescribed between the inlet and outlet while no-slip conditions are prescribed on the solid boundaries.

The solid-fluid interaction coefficients are fixed at $G_{1 s}=0.5$ and $G_{2 s}=-0.5$. The inter-molecular interaction coefficient $G_{12}$ is varied in such a way that spinodal decomposition does not emerge on the mixture; therefore, the system is described by one thermodynamic phase rather than two coexisting phases. This results in the distribution of fluid-1 molecules around fluid-2 molecules and vice versa.

Figure 4.1 shows the scaled density contours of both fluids when $G_{12}=1.0$ and relaxation times are fixed. Figure $4.1(\mathrm{a})$, which presents $\rho_{1}$ normalized by its initial value, indicates that $\rho_{1}$ decreases as we move closer to the solid walls until it reaches its minimum value at the last row of cells in the fluid domain. This drop in density is attributed to the repulsion force imposed by the solid nodes toward fluid-1 particles. It is apparent that this conduct is symmetric around the center-line of the channel except the regions close to the inlet and outlet, due to the discontinuity of the computational domain.

Figure 4.1(b) provides the normalized $\rho_{2}$ contour plot. The density increases as we approach the walls until attaining its maximum near the solid boundaries. This opposite behavior is caused by the attraction forces which concentrate fluid-2 in the vicinity of the solid nodes.

Fig 4.2 shows the effect of variation in $G_{12}$ on the density profiles across the 
channels, along the mid line. Five values of $G_{12}$ from 0.0 to 1.0 in 0.25 increments are examined. For all cases of $G_{12}$, a slight difference is noticed in the value of $\rho_{1}$ in the bulk region away from the walls. However, the profiles of $\rho_{1}$ diverge as one moves towards the walls. The scenario is similar with $\rho_{2}$ but with a substantial deviation in the interior area of the channel. Obviously, the augmentation of cross interaction force would mitigate the strength of repulsion force exerted by the walls on the adjacent particles of fluid-1. For this reason, the maximum drop in $\rho_{1}$ is reduced from about $18 \%$ to $8 \%$ which is reflected to interior nodes where the density approaches its initial value. In contrast, the rise in $G_{12}$ would enhance the attraction force leading to an increase in $\rho_{2}$ from $10 \%$ in the absence of cross interaction to $40 \%$ when $G_{12}=1.0$. Accordingly, the interior zone would experience a drop in $\rho_{2}$ from $3 \%$ to $10 \%$. 


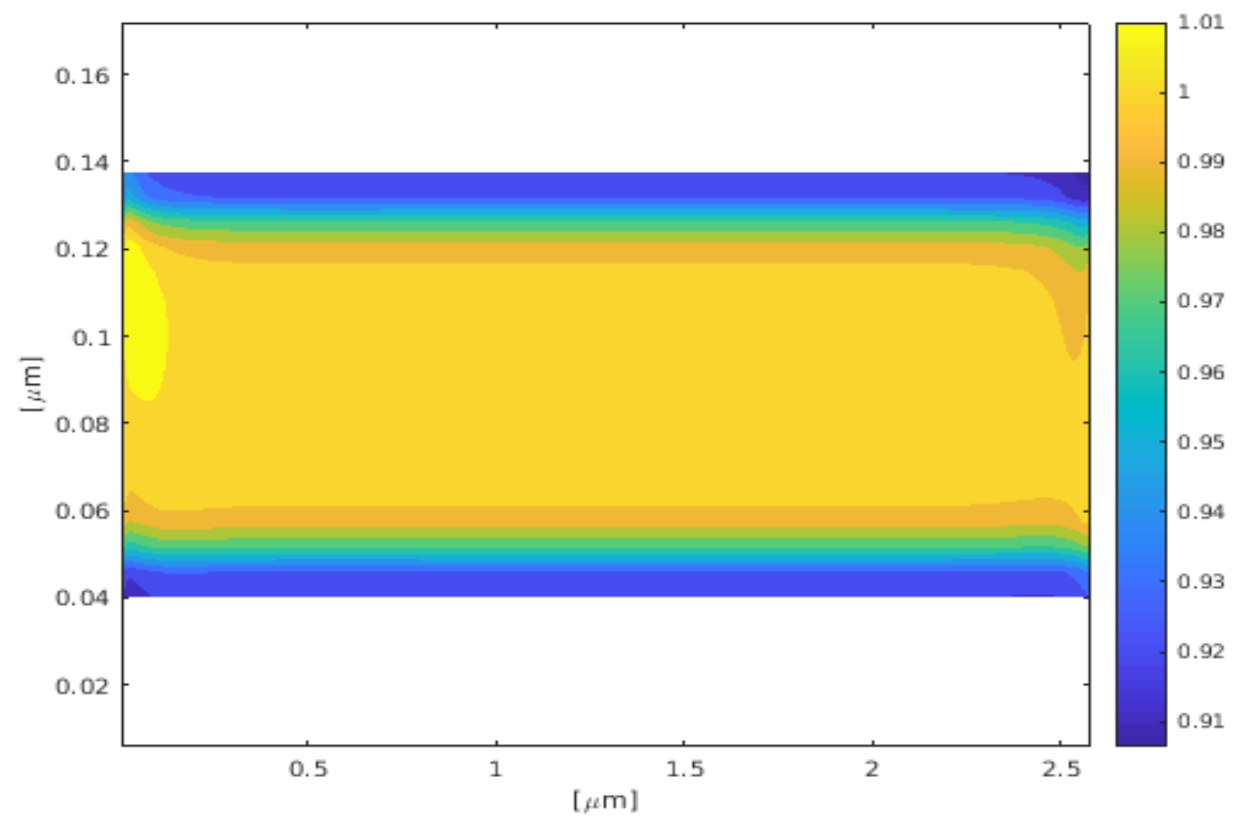

(a)

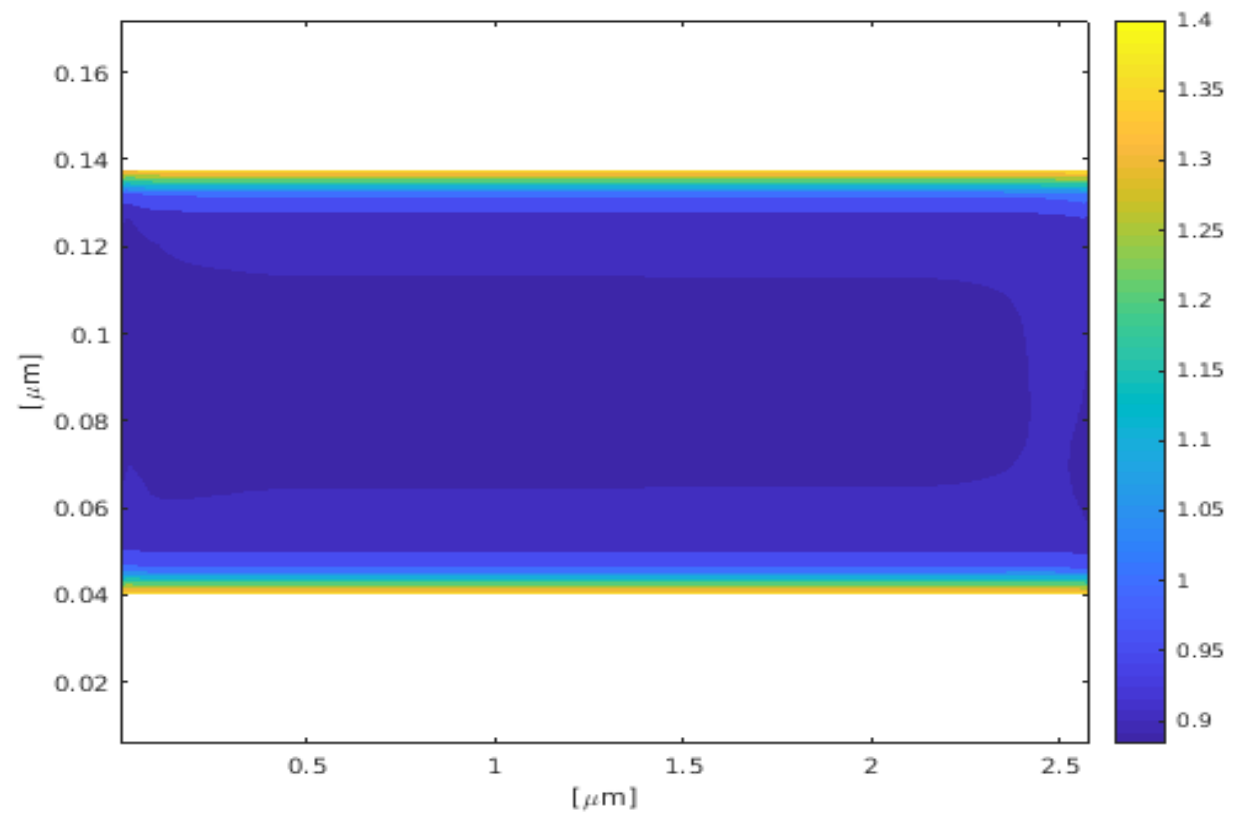

(b)

Figure 4.1: Scaled densities of a binary fluid mixture components at $G_{1 \mathrm{~s}}=0.5$ (low affinity), $G_{2 s}=-0.5$ (high affinity) and cross interaction parameter, $G_{12}=1.00$ when $\tau_{1}=\tau_{2}=0.75 .(\mathrm{a})-\rho_{1}^{*}=\frac{r h o_{1}}{r h o_{1}^{i n i t}}$ and $(\mathrm{b})-\rho_{2}^{*}=\frac{\rho_{2}}{\rho_{2}^{\text {init }}} . \rho_{1}^{*}$ decreases near the walls due to repulsion while $\rho_{2}^{*}$ increases near the walls due to attraction. The wall effect does not exceed $20[\mathrm{~nm}]$ and the densities far away from the walls are constant. 


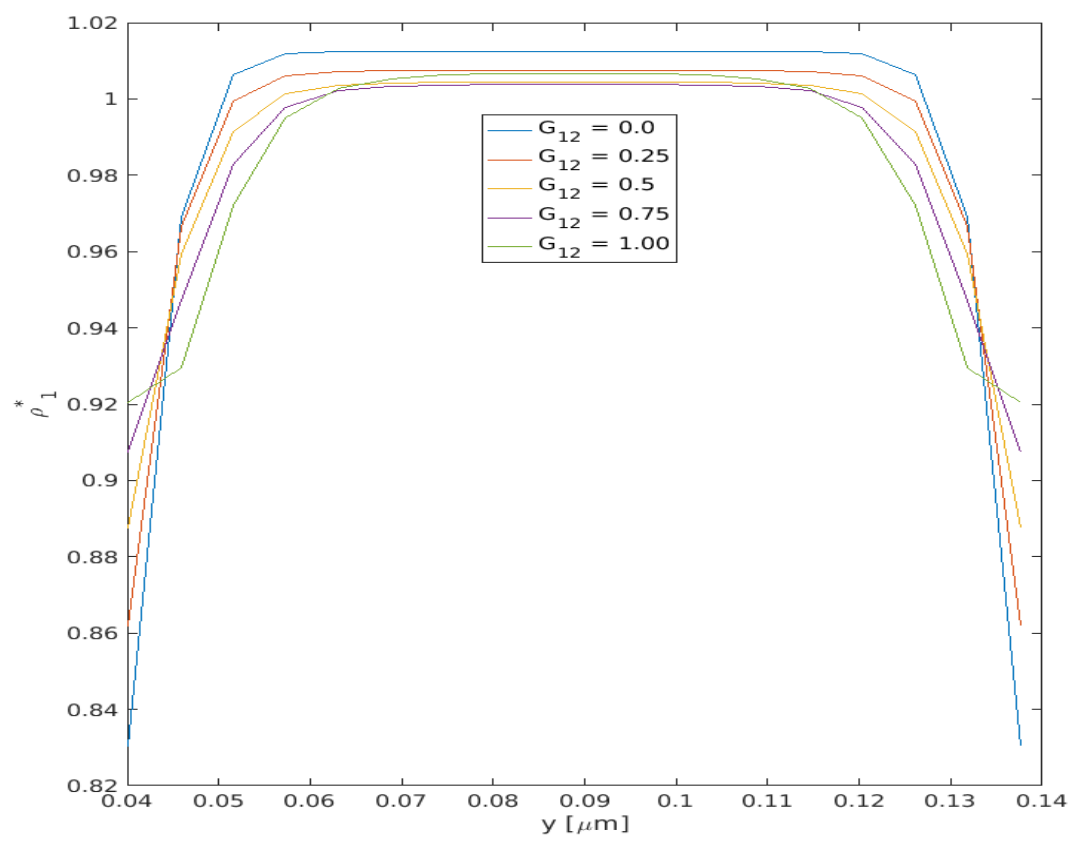

(a)

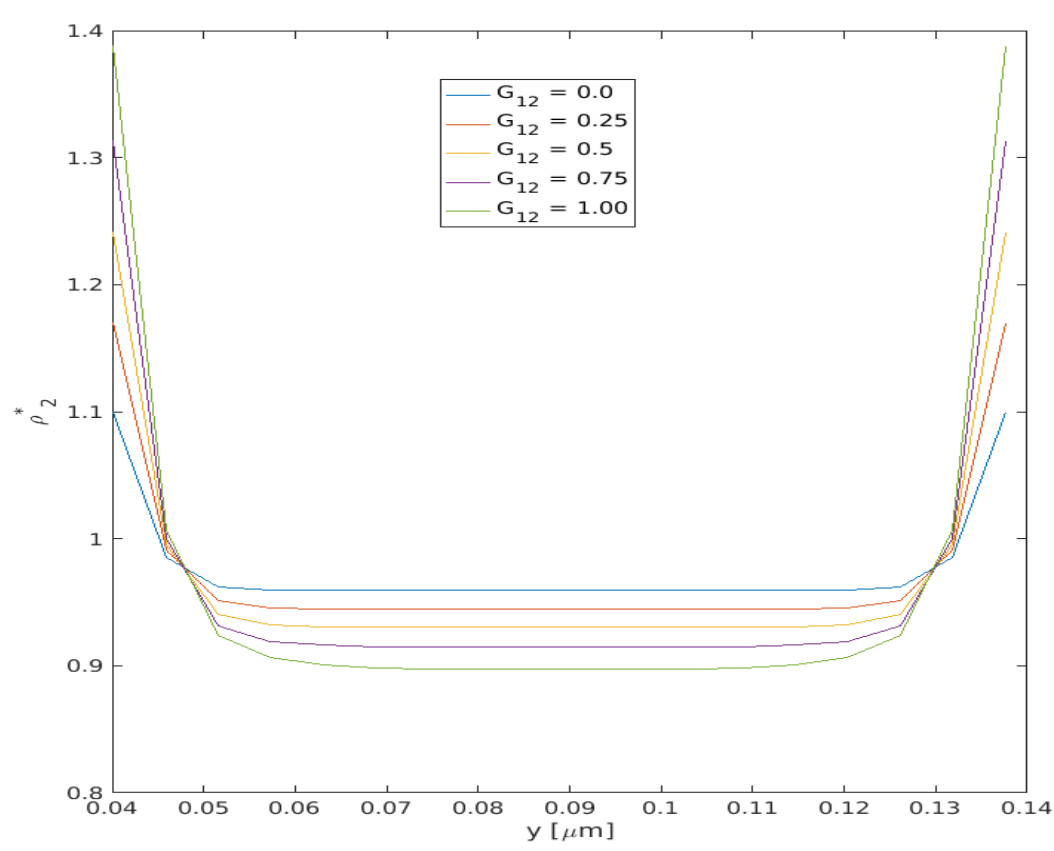

(b)

Figure 4.2: Effect of cross interaction parameter, $G_{12}$, on densities of both fluids at $G_{1 s}=0.5$, and $G_{2 s}=-0.5$ when $\tau_{1}=\tau_{2}=0.75$. (a) $-\rho_{1}^{*}=\frac{r h o_{1}}{r h o_{1}^{i n i t}}$ and (b)- $\rho_{2}^{*}=\frac{\rho_{2}}{\rho_{2}^{i n i t}}$ in the middle of channel. Increasing $G_{12}$ reduces the dispersion of $\rho_{1}^{*}$ and concentrates $\rho_{2}^{*}$ near the walls. 


\subsection{Solid-Fluid Interaction Coefficients Effect}

To understand the surface forces effect on both fluids, the cross interaction coefficient $G_{12}$ is fixed at 0.5 while the solid-fluid interaction coefficients, $G_{1 s}$ and $G_{2 s}$, are varied from -1.0 to 1.0 in 0.5 increments. The borders of this variation stand for the high attraction and repulsion respectively. By this means the affinity of the solid wall to either fluid is controlled.

By setting $G_{2 s}$ to 0.0 and changing $G_{1 s}$ of the upper and lower walls, it is possible to maintain both walls neutral to fluid-2 and of variable affinity to fluid-1. As Figure 4.3 displays, fluid-1 is expelled away from the top and bottom of the channel due to the lack of affinity between solid and fluid particles. On the other hand, fluid-2 is driven towards the walls as a result of the cross interaction force applied by fluid-1, which is also visible in the middle of the channel. As in the previous section, the density distribution is uniform along the channel when subject to wetting conditions in the transverse direction.

The quantitative effect of $G_{1 s}$ on densities along the mid-line of the channel is shown in Fig.4.4. Altering $G_{1 s}$ from -1.0 to 1.0 influences $\rho_{1}$ near the solid surfaces and

over the interior area. Negative values of $G 1 s$, denoting a stronger affinity between fluid-1 and the solid material, concentrate fluid-1 near the wall and de-concentrate it internally. In contrast, positive values of $G_{1 s}$ denoting non-affinity between fluid-1 and the solid material which disperses fluid-1 from the walls and concentrates it at the middle. A $25 \%$ excess in $\rho_{1}$ is observed near the walls when $G_{1 s}=-1.0$ while a reduction of $20 \%$ is observed when $G_{1 s}=1.0$. Furthermore, the change in the middle of the channel does not exceed $-7 \%$ and $2 \%$ as noted in the the previous cases of $G_{1 s}$. Figure 4.4 (b) provides the profiles of $\rho_{2}$. As there is repulsion everywhere except near the walls which are neutral to fluid-2, $\rho_{2}$ profiles exhibit only a slight change in 
profile shape for the entire range of $G_{1 s}$ with no inflection in the trend as observed with $\rho_{1}$.

Figures (4.5) and (4.6) show the effect of changing $G_{2 s}$. In this case $\rho_{2}$ profiles are significantly affected by the choice of $G_{2 s}$ where as $\rho_{1}$ is slightly impacted. About a $35 \%$ increase in $\rho_{2}$ is observed near the solid boundaries when $G 2 s$ is -1.0 . A $15 \%$ reduction is observed when $G_{2 s}$ is 1.0 . The sharp transition near the wall in Figure 4.6 (a) are not observed in other profiles and may be due to the self attraction parameter, $G 11=-0.25$, which strengthens the cross repulsion thus decreasing $\rho_{1}$ wherever fluid-2 is more condensed except at the last node where the cross repulsion is missing from the wall. 


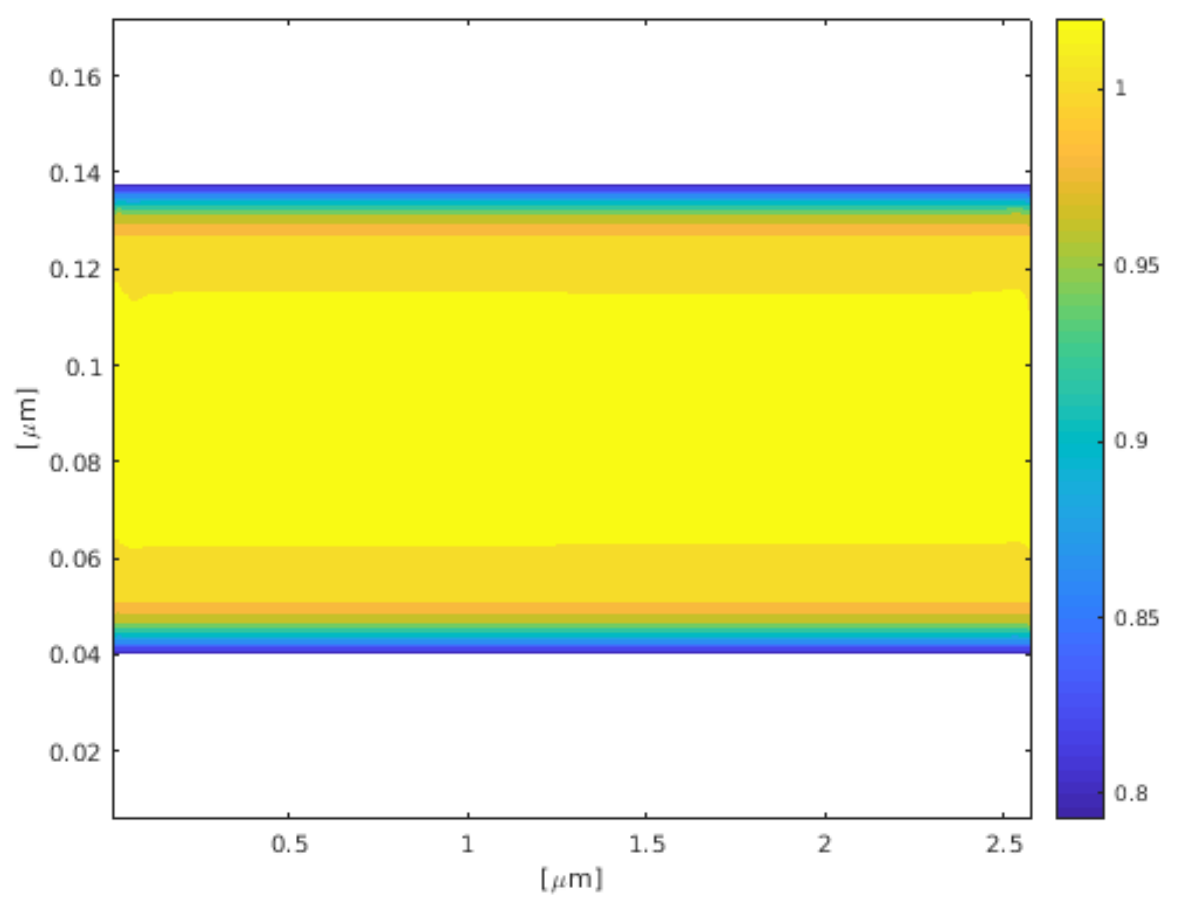

(a)

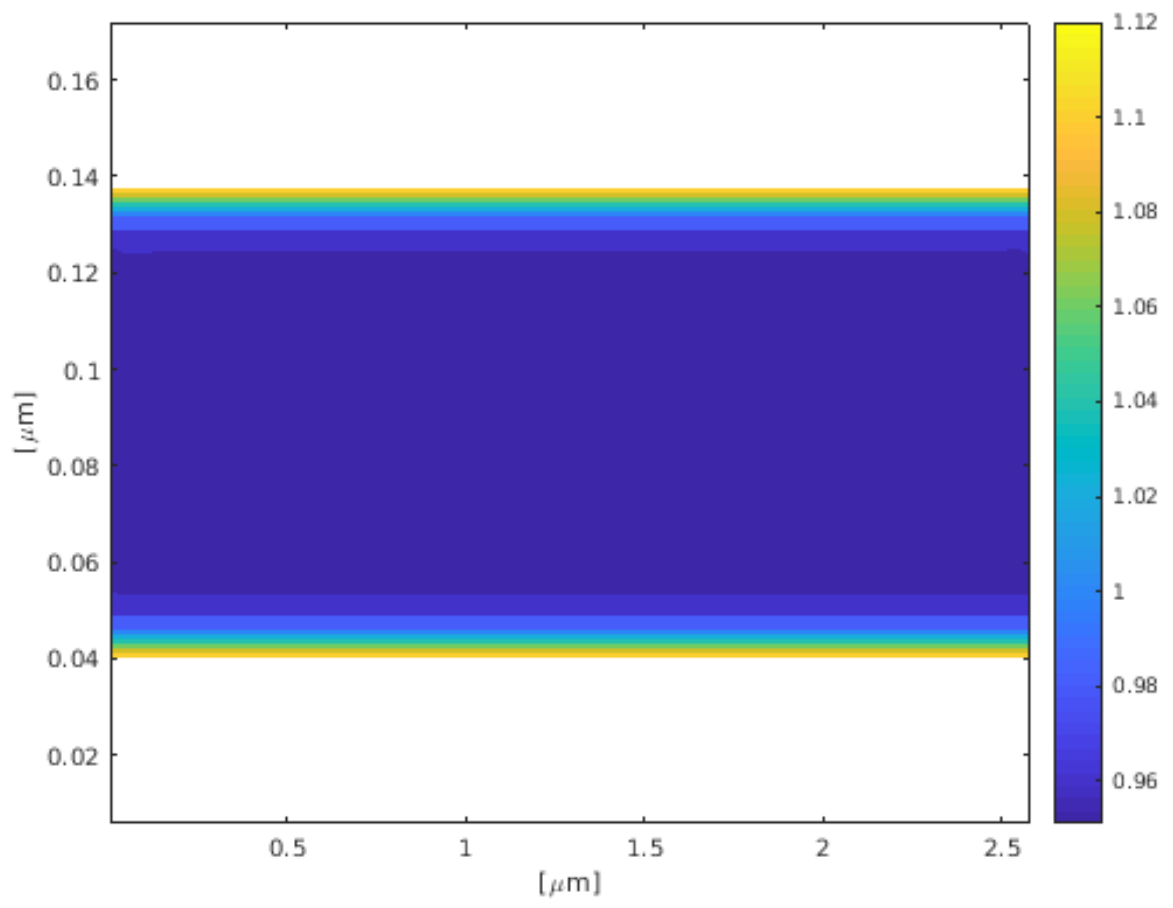

(b)

Figure 4.3: Scaled densities of both fluids at $G_{1 s}=1.0, G_{2 s}=0.0$, and $G_{12}=0.5$ when $\tau_{1}=\tau_{2}=0.75$. (a) $-\rho_{1}^{*}=\frac{r h o_{1}}{r h o_{1}^{i n i t}}$ and $(\mathrm{b})-\rho_{2}^{*}=\frac{\rho_{2}}{\rho_{2}^{\text {init }} \text {. }}$ 


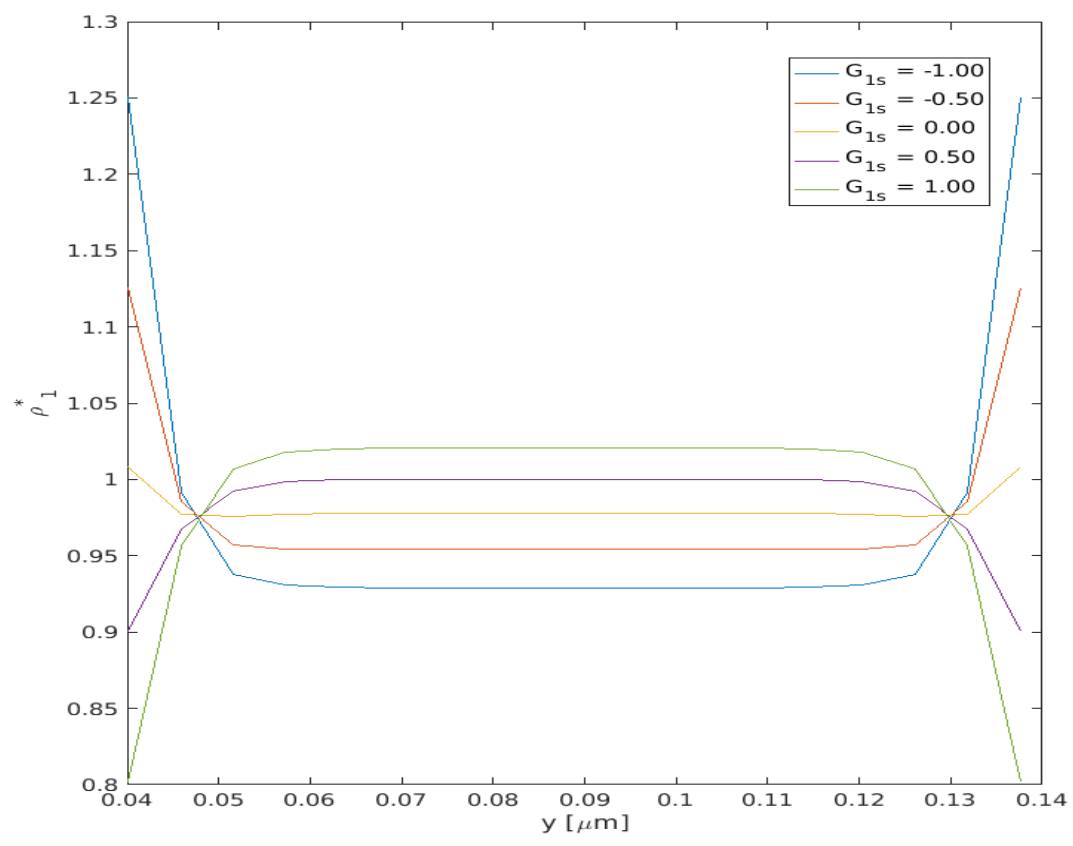

(a)

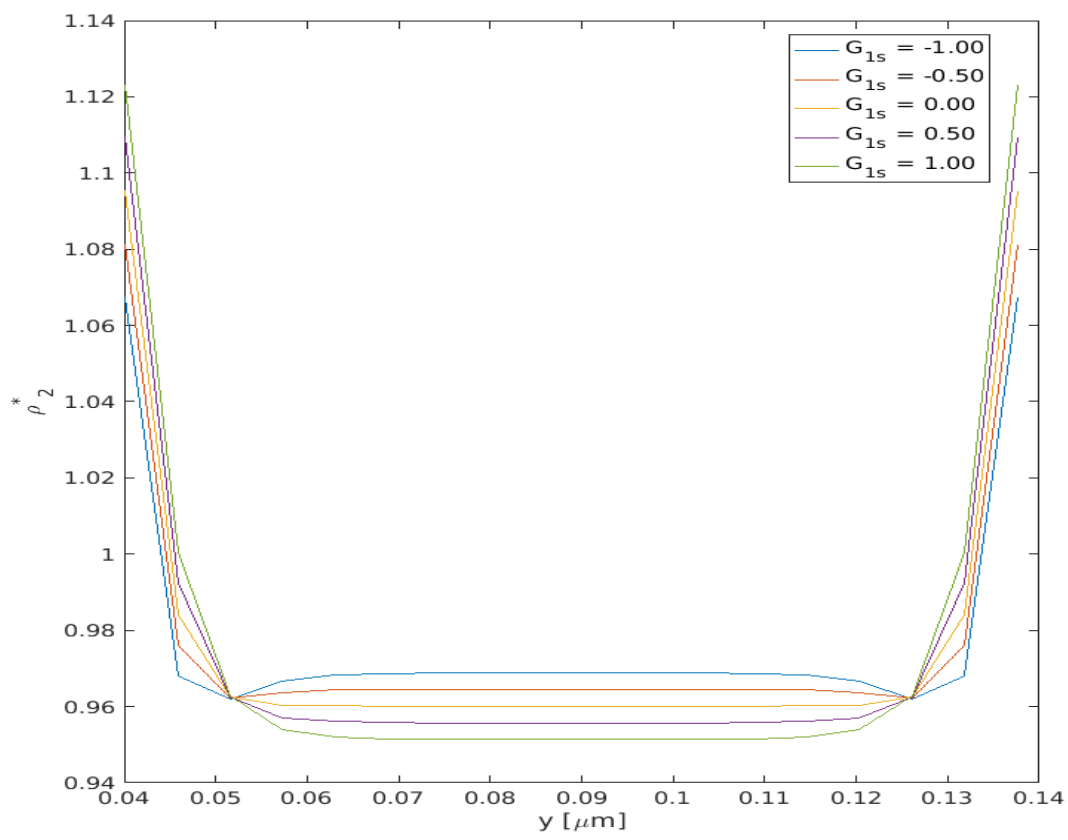

(b)

Figure 4.4: Effect of Solid-Fluid interaction parameter, $G_{1 s}$, on densities of both fluids in the middle of channel at $G_{2 s}=0.0$, and $G_{12}=0.5$ when $\tau_{1}=\tau_{2}=0.75$. (a)$\rho_{1}^{*}=\frac{r h o_{1}}{r h o_{1}^{\text {init }}}$ and $(\mathrm{b})-\rho_{2}^{*}=\frac{\rho_{2}}{\rho_{2}^{\text {init }}} \cdot \rho_{1}$ is directly affected near the wall while $\rho_{2}$ is slightly changed due to $G_{1 s}$ change. 


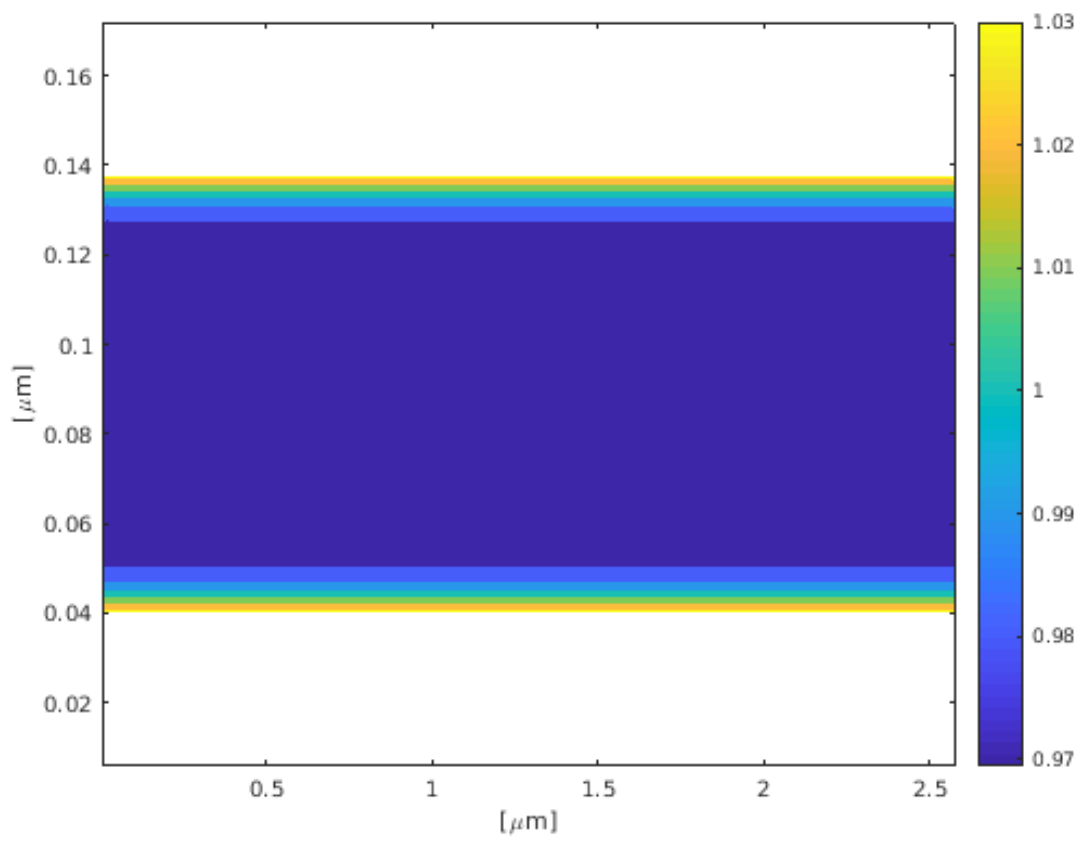

(a)

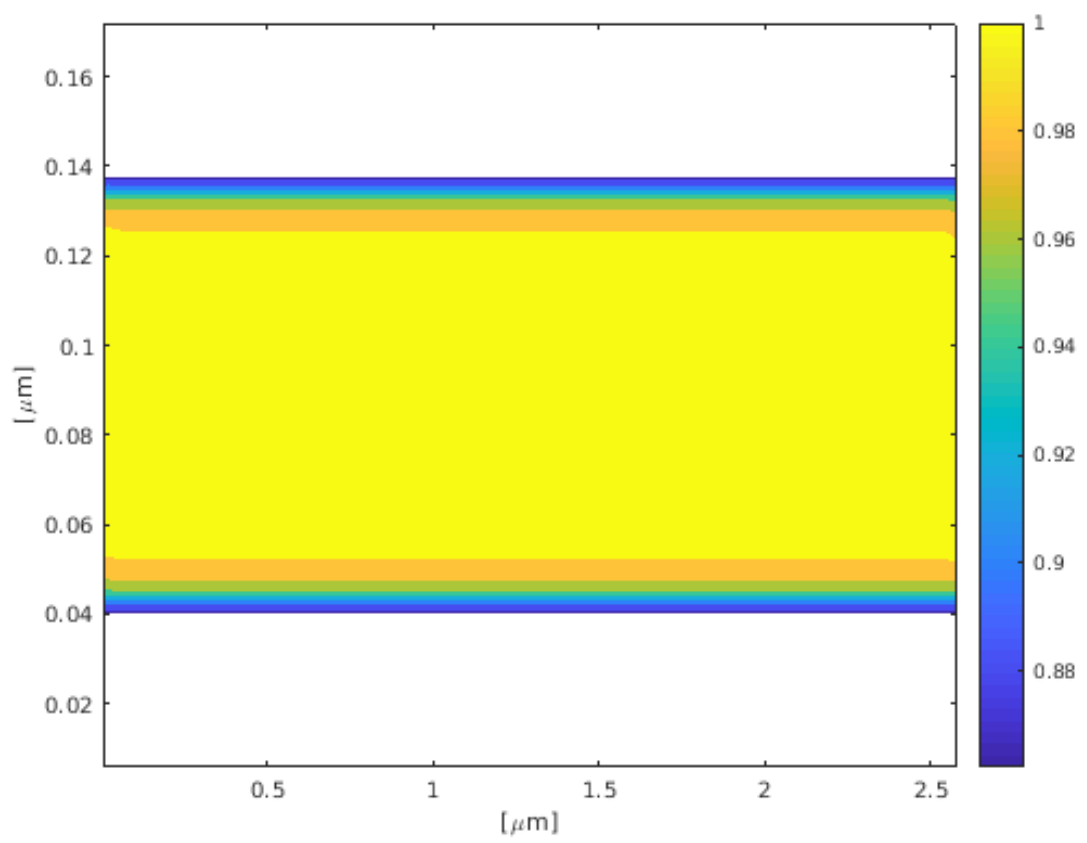

(b)

Figure 4.5: Scaled densities of both fluids at $G_{2 s}=1.0, G_{1 s}=0.0$, and $G_{12}=0.5$ when $\tau_{1}=\tau_{2}=0.75$. (a) $-\rho_{1}^{*}=\frac{r h o_{1}}{r h o_{1}^{i n i t}}$ and $(\mathrm{b})-\rho_{2}^{*}=\frac{\rho_{2}}{\rho_{2}^{\text {init }}}$. Fluid-1 is slightly forced to the wall while fluid-2 is repelled from the wall. 


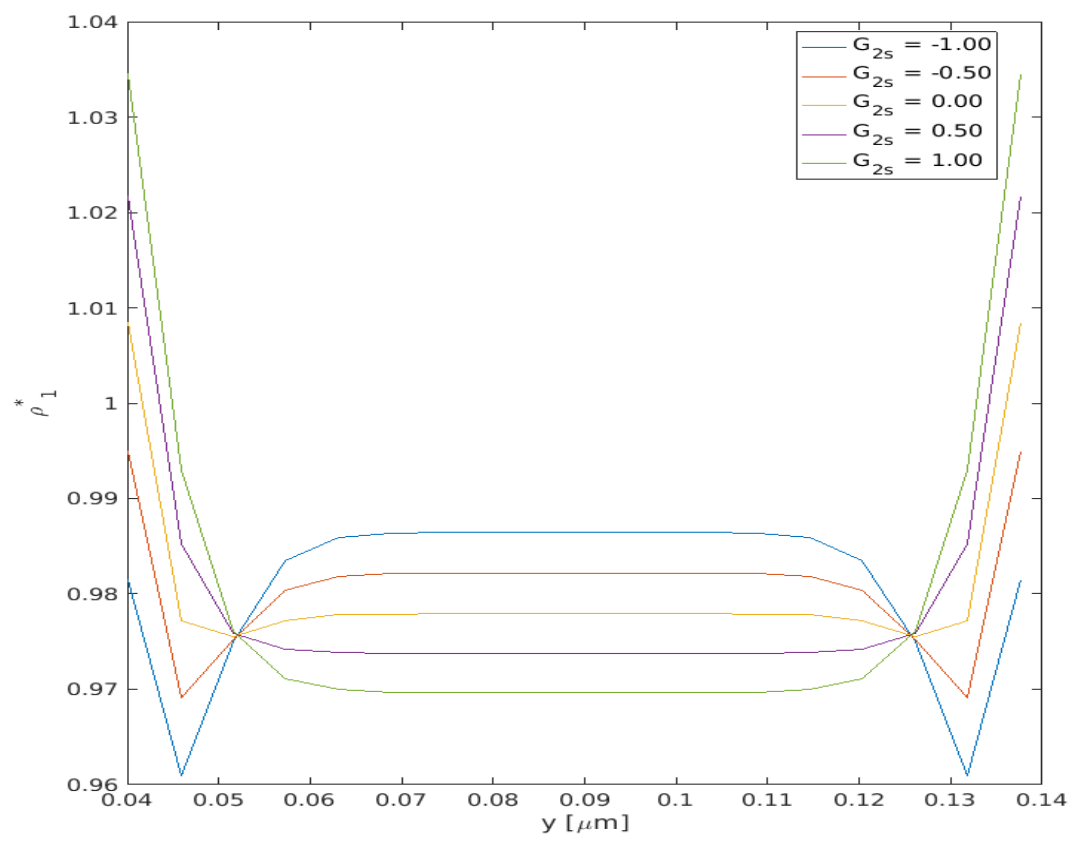

(a)

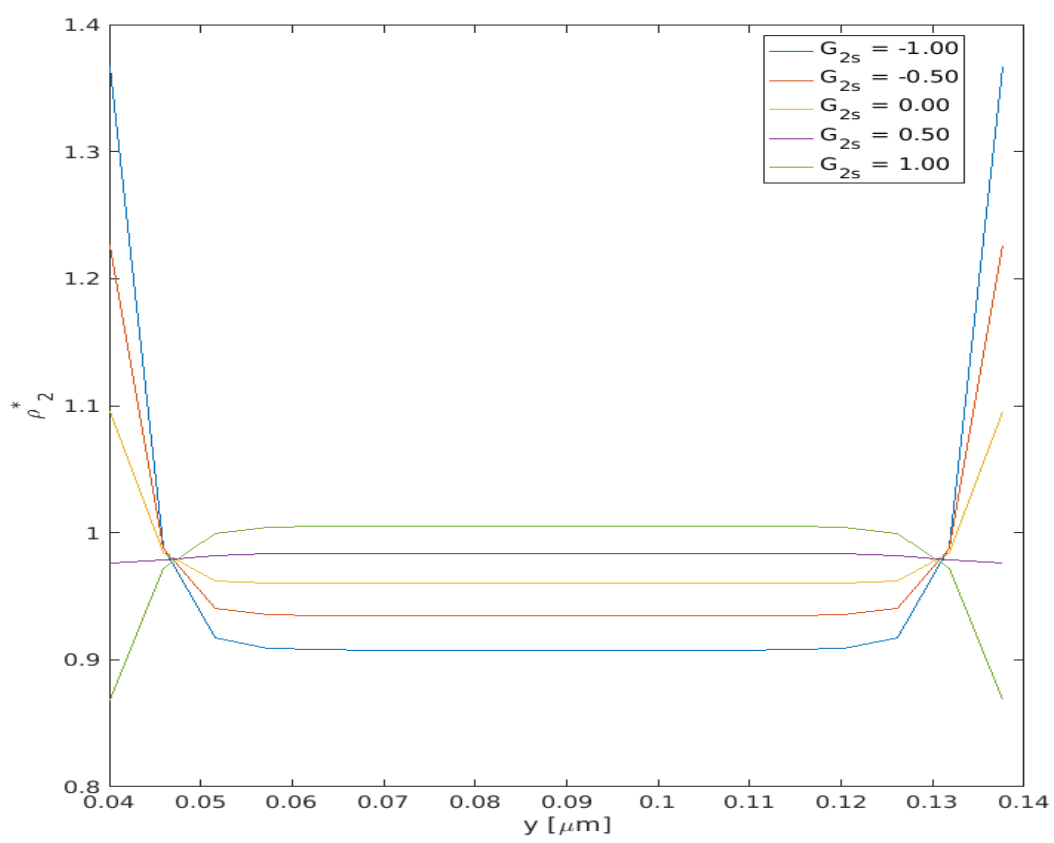

(b)

Figure 4.6: Effect of Solid-Fluid interaction parameter, $G_{2 s}$, on densities of both fluids in the middle of channel at $G_{1 s}=0.0$, and $G_{12}=0.5$ when $\tau_{1}=\tau_{2}=0.75$. (a)$\rho_{1}^{*}=\frac{r h o_{1}}{r h o_{1}^{i n i t}}$ and (b)- $\rho_{2}^{*}=\frac{\rho_{2}}{\rho_{2}^{\text {init }}}$. Fluid-1 is slightly affected by $G_{2 s}$ while fluid-2 is noticeably impacted near the wall. 


\subsection{Relaxation Times Effect}

As we mentioned in subsection 3.2.1 the distribution functions are relaxed to their equilibrium state after the collision process. The time interval of this process is controlled by the relaxation time $\tau$. The choice of $\tau$ is directly related to the viscosity and the spatial and temporal steps of the physical system. In this section we focus on the effects of changing the relaxation times of both mixture components, $\tau_{1}$ and $\tau_{2}$, while maintaining the remaining properties constant.

Three cases are tested by fixing $\tau$ of one component and running the simulation at $\tau=0.6,0.8$, and 1.0 for the other component under constant mixture viscosity. The density contour plots in Figure 4.7 and Figure 4.9 display similar distributions shapes for both densities even for different $\tau_{1}$ and $\tau_{2}$. However, fixing one of them, say $\tau_{2}$, and altering the other, $\tau_{1}$, impacts the the overall size of the channel as shown in the density profiles in Figure 4.8. To explain why, shorter relaxation times are associated with higher temperatures of the system [14]; therefore, from the fundamentals of thermodynamics the distance between molecules increases [85]. In other words, the lattice spacing is increased by reducing either one of the relaxation times which in turn reduces the overall size of the channel for a constant number of cells. Microscopically the mean free path is increased by raising the temperature which increases the kinetic energy of the colliding molecules. Increasing the rate of collisions experienced by fluid particles shortens the relaxation time. The magnitudes of the interaction forces are not affected by the selection of the relaxation times thus no intensification or reduction is noticed in density profiles. However, the range of the intermolecular forces is extended at least $250 \%$ when either one of the relaxation times is decreased from 1.0 to 0.6 . 


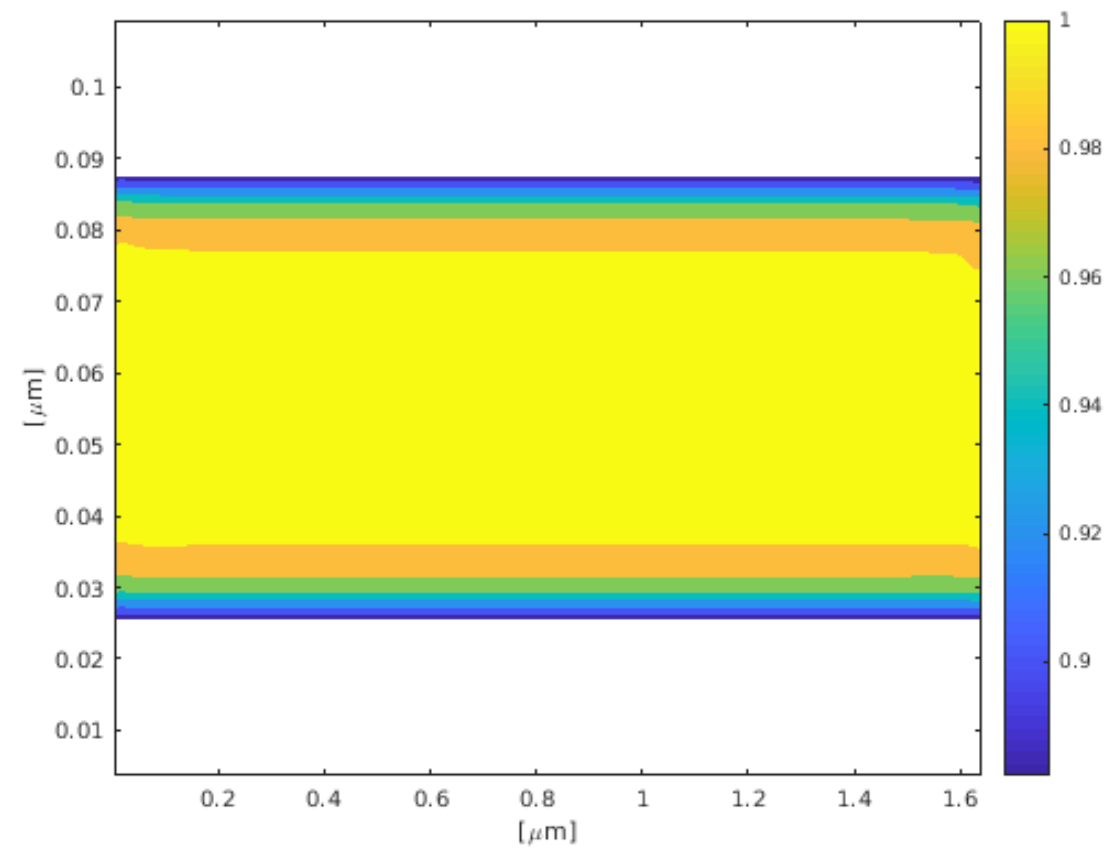

(a)

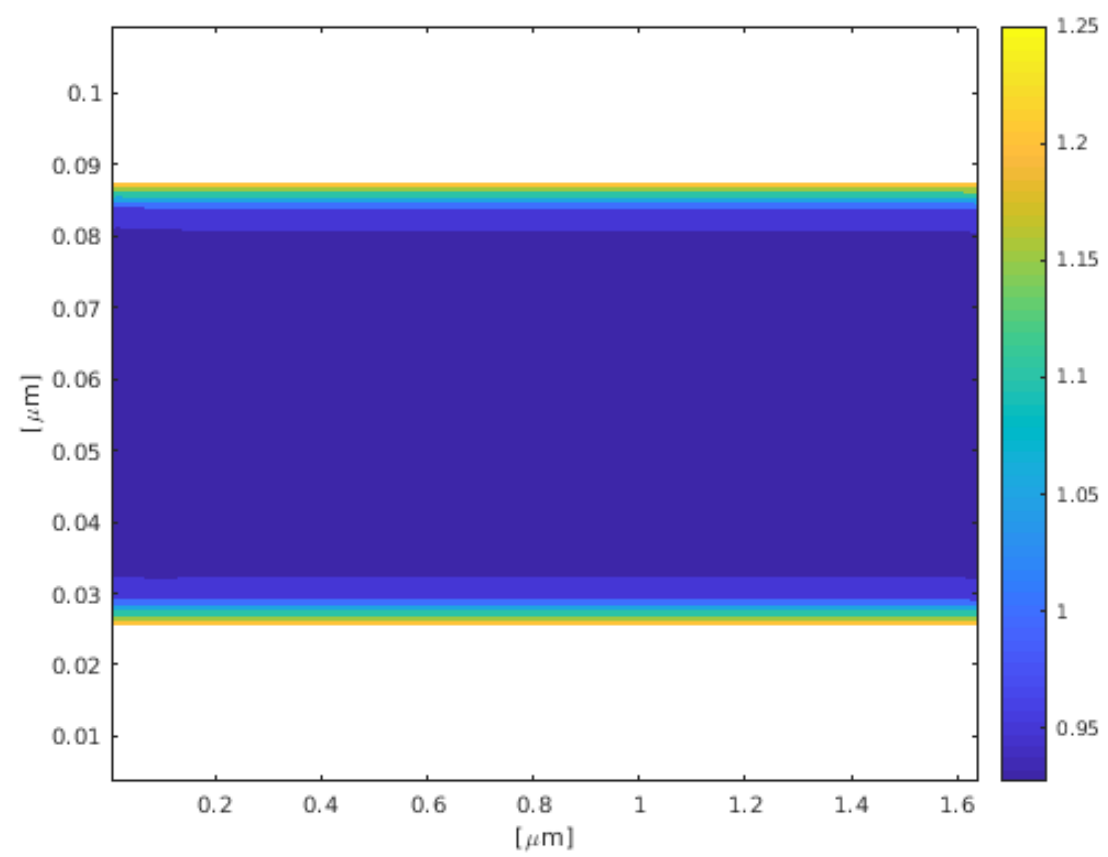

(b)

Figure 4.7: Scaled densities of both fluids at relaxation time $\tau_{1}=1.0$ when $\tau_{2}=$ $0.75, G_{12}=0.5, G_{1 s}=0.5$ and $G_{2 s}=-0.5$.(a) $-\rho_{1}^{*}=\frac{r h o_{1}}{r h o_{1}^{\text {init }}}$ and (b)- $\rho_{2}^{*}=\frac{\rho_{2}}{\rho_{2}^{\text {init }}}$. 


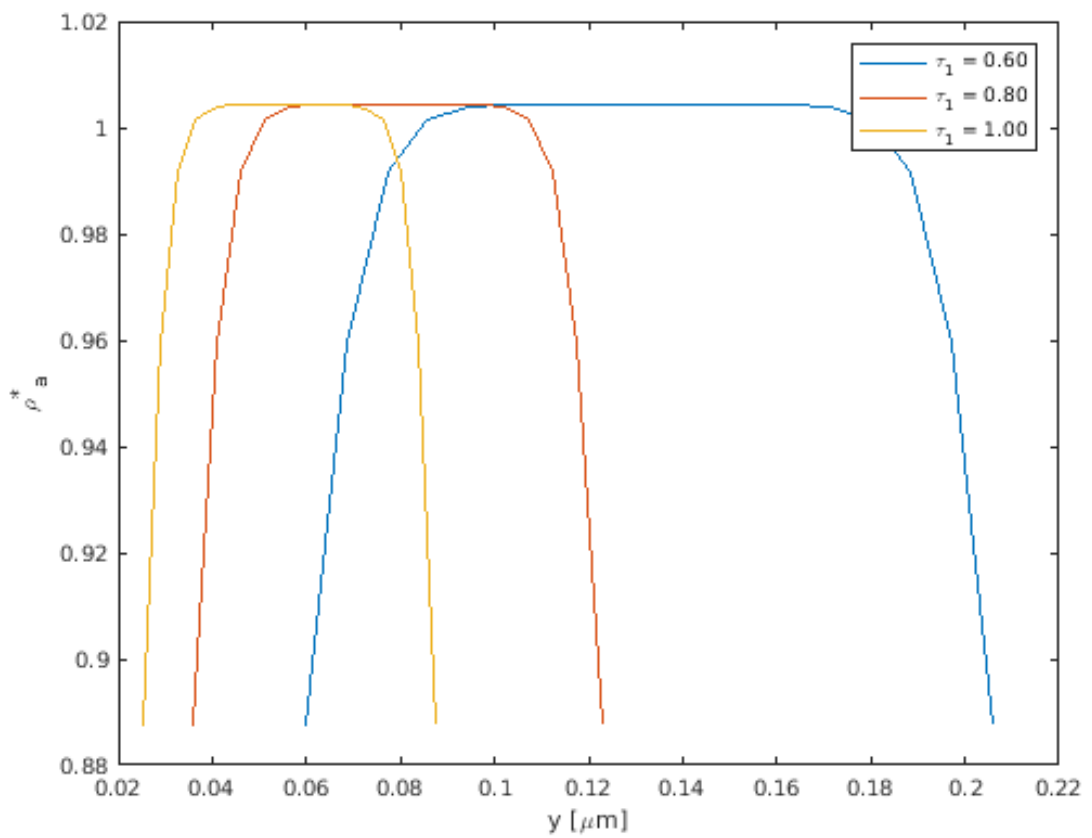

(a)

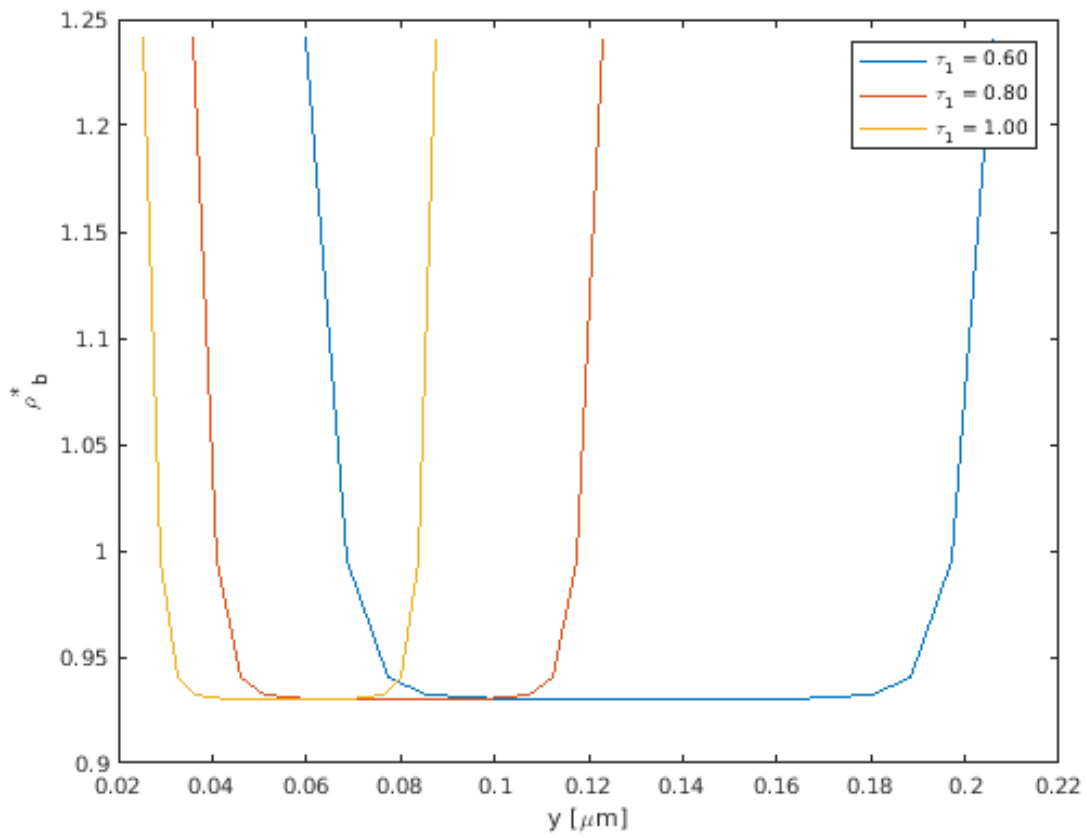

(b)

Figure 4.8: Effect of relaxation time of fluid-1, $\tau_{1}$, on densities of both fluids in the middle of channel at $\tau_{2}=0.75, G_{12}=0.5, G_{1 s}=0.5$ and $G_{2 s}=-0.5$. (a)- $\rho_{1}^{*}=\frac{r h o_{1}}{r h o_{1}^{\text {init }}}$ and $(\mathrm{b})-\rho_{2}^{*}=\frac{\rho_{2}}{\rho_{2}^{\text {init }}}$. The length scale over which the density varies is extended when $\tau_{1}$ is decreased. 


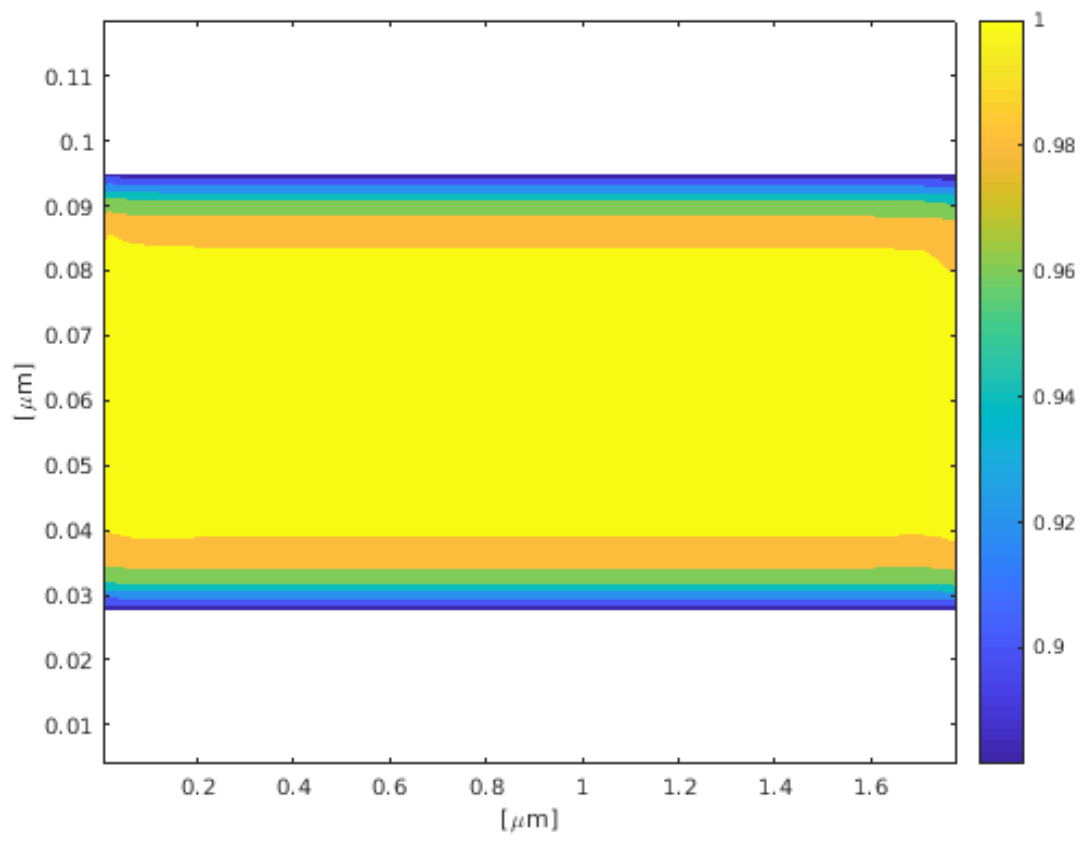

(a)

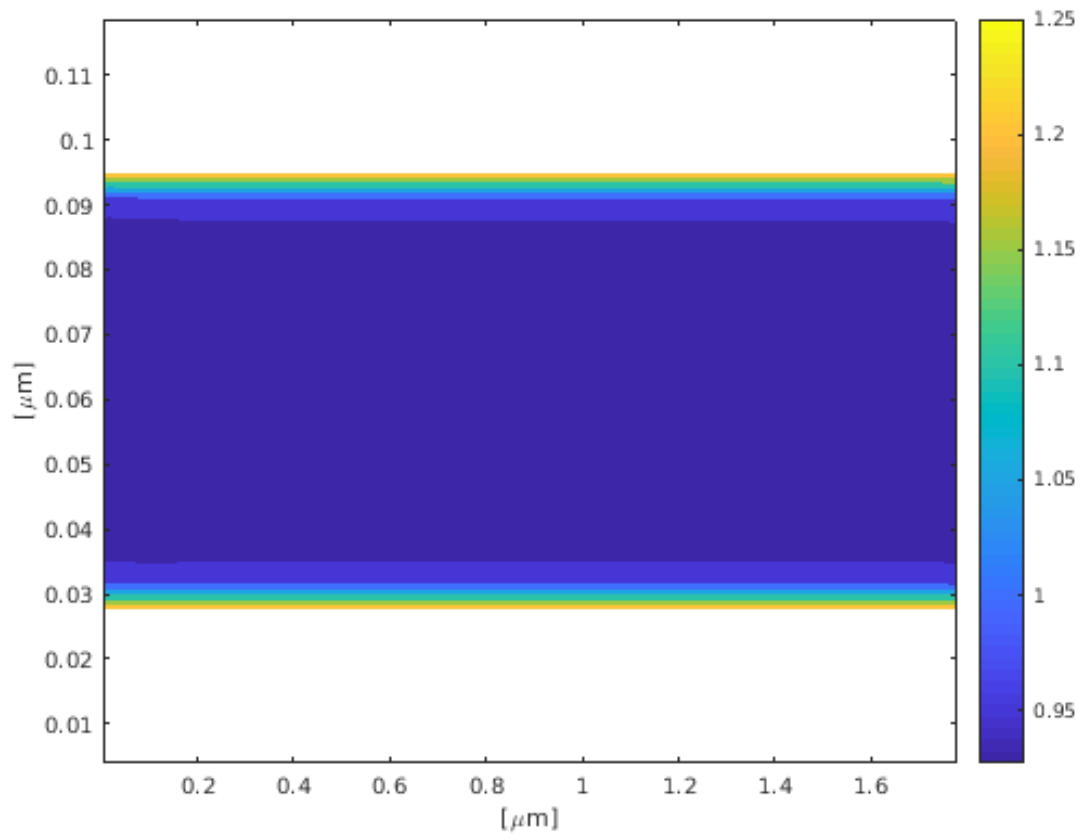

(b)

Figure 4.9: Scaled densities of both fluids of both fluids at relaxation time, $\tau_{2}=1.0$ when $\tau_{1}=0.75, G_{12}=0.5, G_{1 s}=0.5$ and $G_{2 s}=-0.5$. (a)- $\rho_{1}^{*}=\frac{r h o_{1}}{r h o_{1}^{i n i t}}$ and (b)- $\rho_{2}^{*}=$ $\frac{\rho_{2}}{\rho_{2}^{i n i t}}$. 


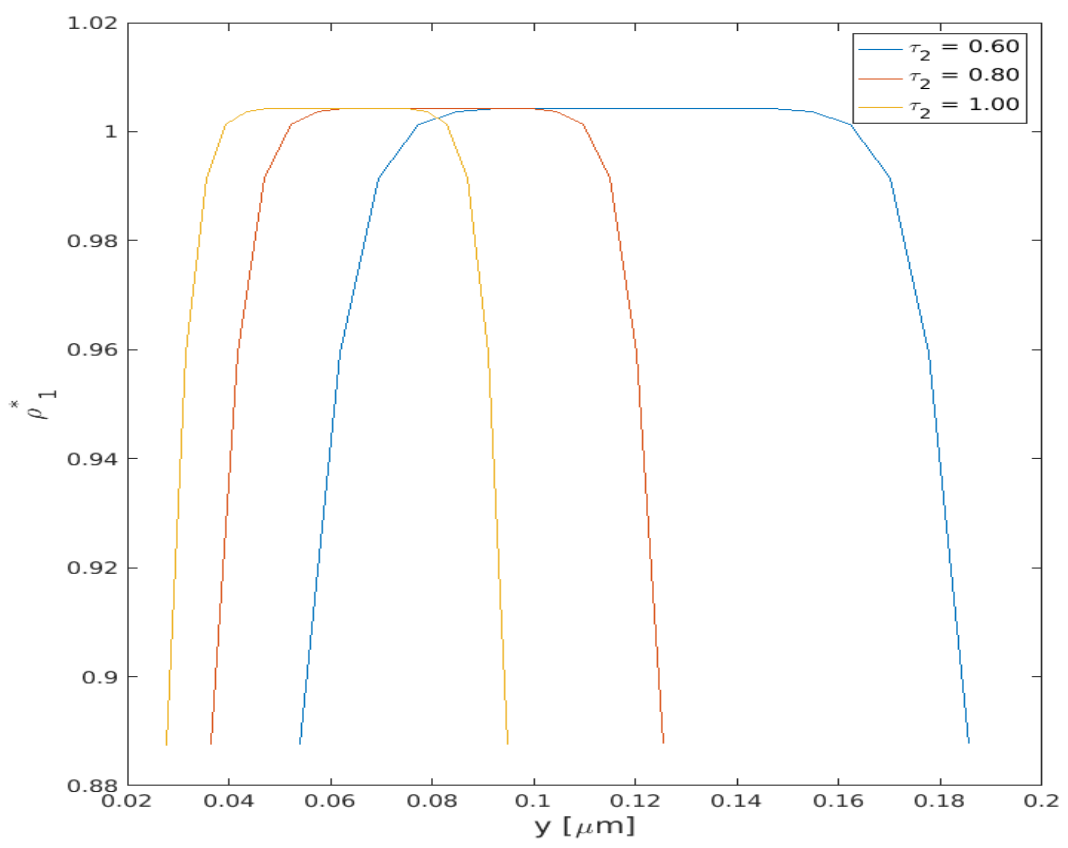

(a)

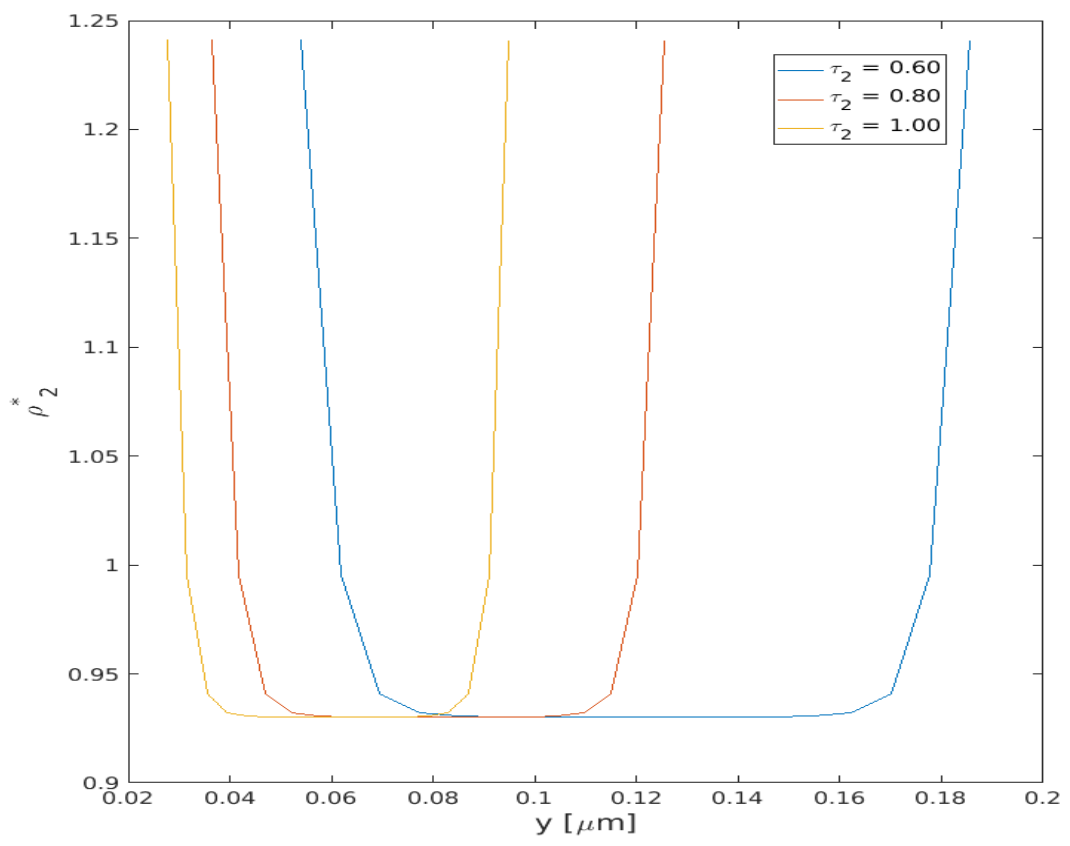

(b)

Figure 4.10: Effect of relaxation time of fluid-2, $\tau_{2}$, on densities of both fluids in the middle of channel at $\tau_{1}=0.75, G_{12}=0.5, G_{1 s}=0.5$ and $G_{2 s}=-0.5$. (a)- $\rho_{1}^{*}=\frac{r h o_{1}}{r h o_{1}^{\text {init }}}$ and (b)- $\rho_{2}^{*}=\frac{\rho_{2}}{\rho_{2}^{i n i t}}$. The length scale over which the density varies is extended when $\tau_{2}$ is decreased. 


\subsection{Height of channel effect}

In the previous section we showed the size of the channel is varied by controlling the spacial step size through manipulation of relaxation times. In this section we show the effect of changing the number of lattices across the channel for fixed step sizes, and thus constant relaxation times. This provides additional control of the channel size.

Five different cases are simulated based on the number of cells in the y-direction, $N_{y}$. The density contours in Fig.4.11 are presented for $N_{y}=25,18$, and 15 when an even repulsion, $G_{1 s}=0.5$, is applied to fluid-1 while an opposite wetting condition, $G_{2 s}=-0.5$, is prescribed for fluid-2. The decrease in $N_{y}$ is translated to a contraction in the internal domain, where the density is almost constant, leaving the regimes near the walls unaffected unless the walls are so close to each other that force entanglement leads to unusual density distributions.

The density profiles in the middle of the channel at variable $N_{y}$ are shown in Figure 4.12. The comparison between $\rho_{1}$ and $\rho_{2}$ indicates that shrinking the size of the channel does not influence the $\rho_{1}$ magnitudes both internally and peripherally except for the case $N_{y}=15$ where the density deviates appreciably from its initial value everywhere. On the other hand, $\rho_{2}$ decreases both inside and near the wall as $N_{y}$ is decreased and the drop is more pronounced for narrower channels. Similar to what was seen with $\rho_{1}$ at $N_{y}=15$, the scaled $\rho_{2}$ diverges from unity which denotes either a weak Galilean invariance due to the insufficient number of nodes in the ydirection or entrapment of the fluid in hidden layers, $\mathrm{dx} / 2$ thick known from the halfway bounce back, located along the walls. If the latter interpretation is true, this means the amount of fluid-1 enclosed near the upper wall is a result of the hard core repulsion from the lower wall and vice versa. The attraction effect on fluid-2 
plays in different way where the concentration regimes are located according to side of attraction.

One more theme can be drawn from Figure 4.12 based on the nature of interactions experienced by each fluid component. The fact that fluid-1 is exposed to repulsion forces from both the solid boundaries and fluid-2, this creates stability in the magnitude of density in most cases of $N_{y}$. Contrarily, fluid-2 responds directly to any change in $N_{y}$ because it is concentrated near the boundaries due to attraction forces. 


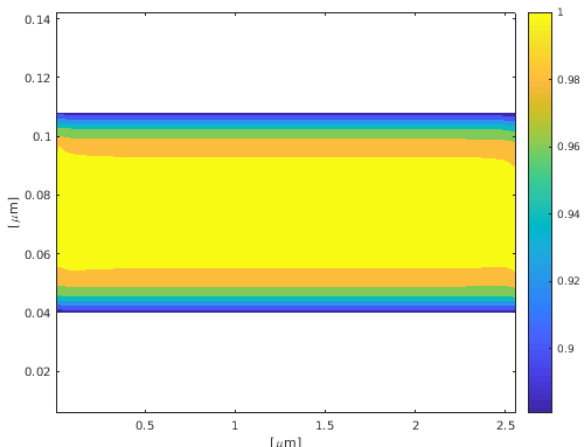

(a) $N_{y}=25$

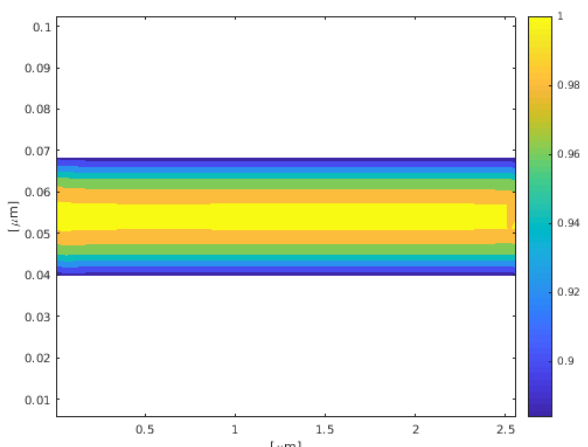

(c) $N_{y}=18$

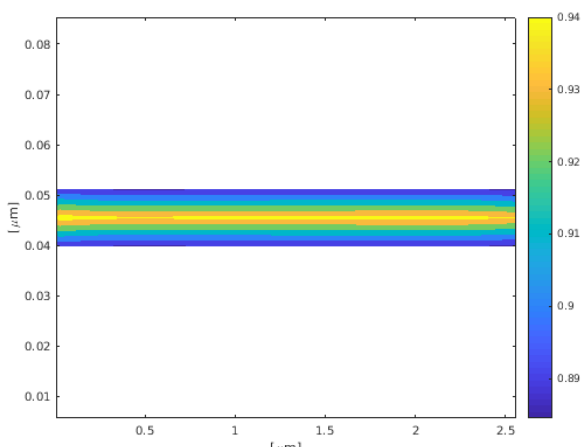

(e) $N_{y}=15$

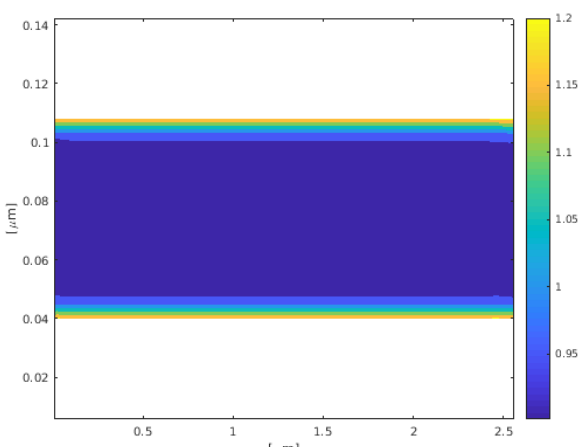

(b) $N_{y}=25$

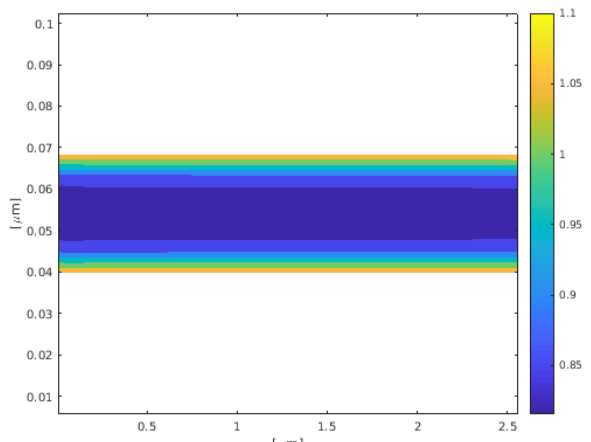

(d) $N_{y}=18$

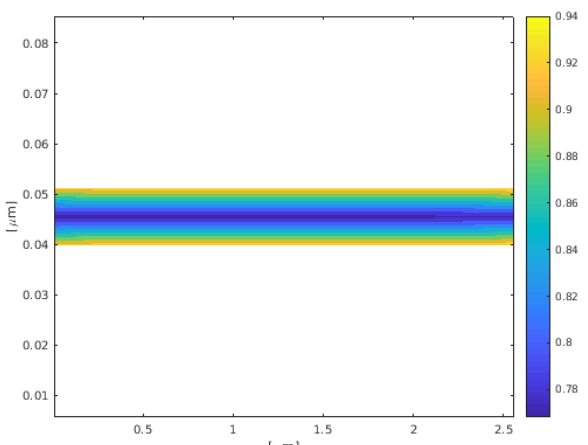

(f) $\stackrel{\mathrm{luml}}{N}_{y}=15$

Figure 4.11: Scaled densities of both fluids((a),(c),(e)- $\rho_{1}^{*}=\frac{r h o_{1}}{r h o_{1}^{i n i t}}$ and (b),(d),(f)$\left.\rho_{2}^{*}=\frac{\rho_{2}}{\rho_{2}^{\text {init }}}\right)$ for different channel heights when $\tau_{1}=\tau_{2}=0.75, G_{12}=0.5, G_{1 s}=0.5$ and $G_{2 s}=-0.5$. 


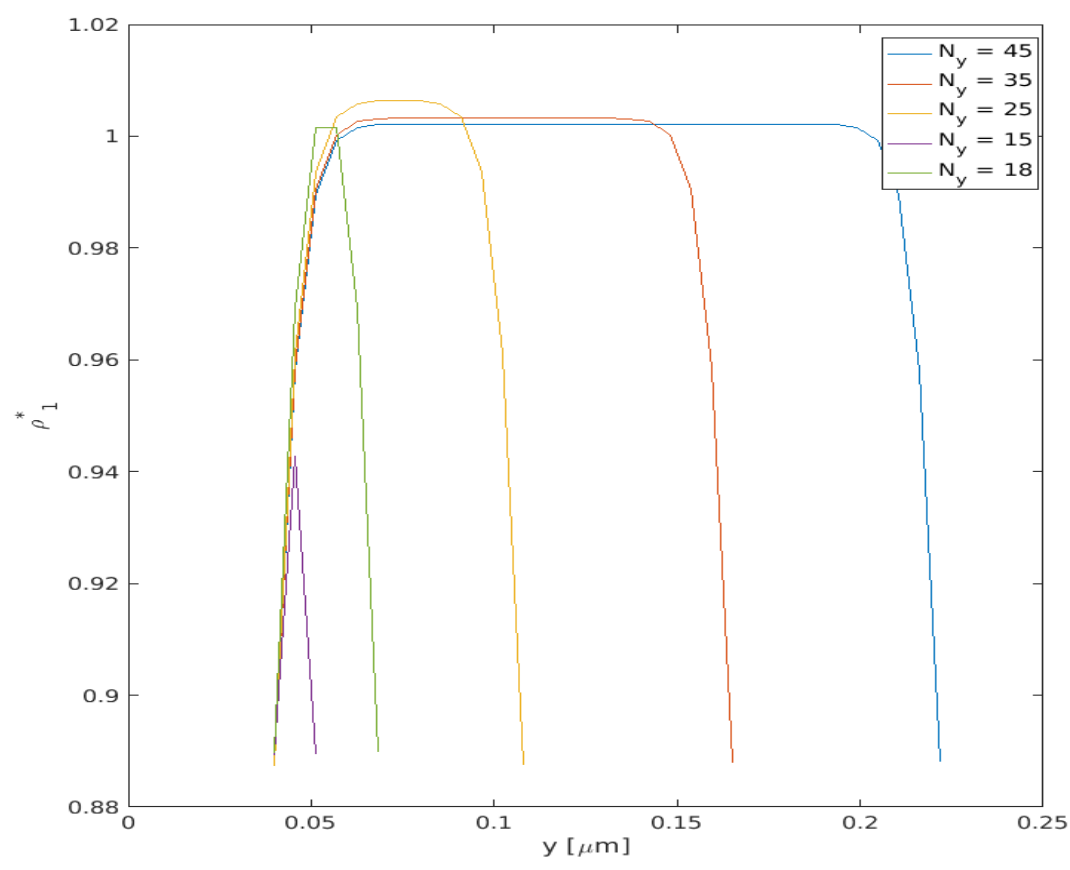

(a)

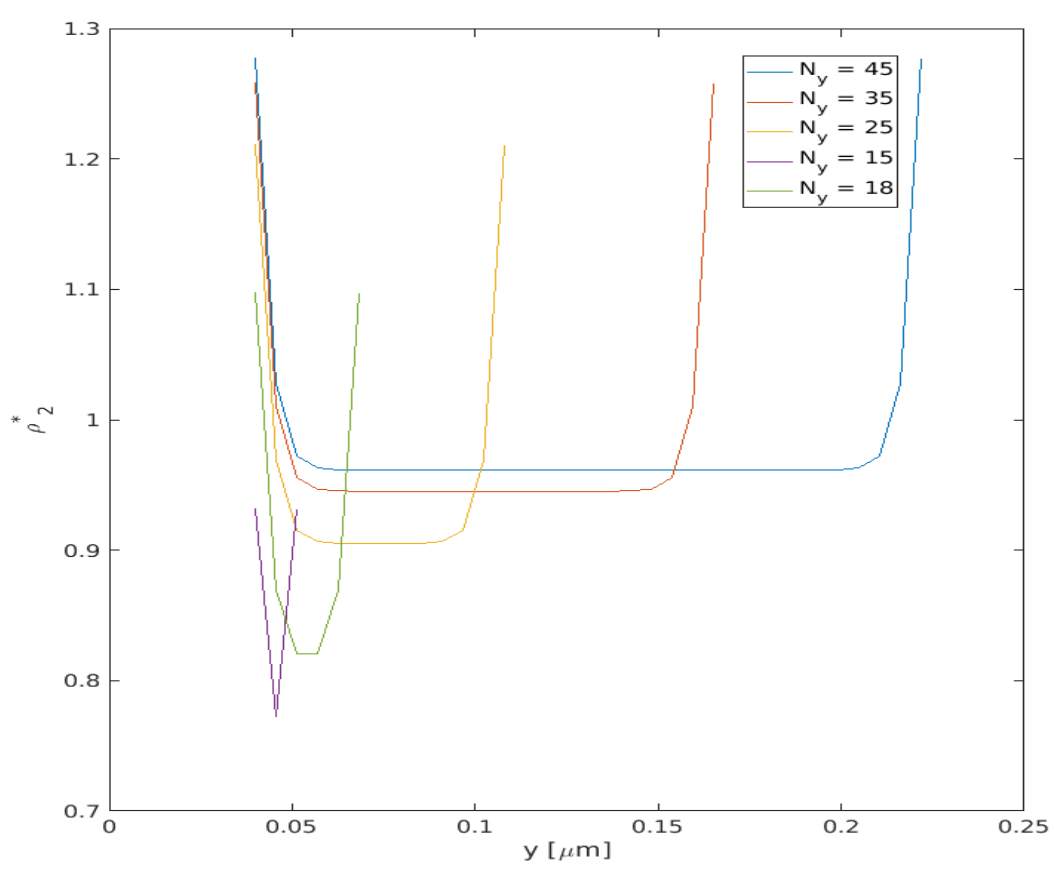

(b)

Figure 4.12: The effect of changing the height of channel by reducing the number of cells on the scaled species densities $\left((\mathrm{a})-\rho_{1}^{*}=\frac{r h o_{1}}{r h o_{1}^{i n i t}}\right.$ and $\left.(\mathrm{b})-\rho_{2}^{*}=\frac{\rho_{2}}{\rho_{2}^{i n i t}}\right)$ when $\tau_{1}=\tau_{2}=$ $0.75, G_{12}=0.5, G_{1 s}=0.5$ and $G_{2 s}=-0.5$. 


\subsection{Uneven Wetting with Variable Heights}

So far we have dealt with channels of even wetting conditions in which both walls are hydrophobic to one species and hydrophilic to the other. In these conditions the first fluid is repelled away from the solid boundaries while the second fluid is attracted to the solid boundaries. Practically, this is not very useful for the separation purposes where two fluids need to be spatially dispersed across the channel such that each fluid would leave the channel on different sides though two outlets. Therefore, in this section uneven wetting conditions are implemented for channels of different heights.

Figure 4.13 shows the density contours for channels with upper wall attracting fluid-1 and repelling fluid-2. Alternatively, the lower wall attracts fluid-2 and repels fluid-1. This is achieved by reversing the signs of $G_{1 s}$ and $G_{2 s}$ when defining the wettabilities of the solid nodes. As a result of this implementation, $\rho_{1}$ has its peak along the upper wall and its lowest level in the vicinity of the lower wall. Alternatively, $\rho_{2}$ reaches its peak close to the lower wall and its minimum in the vicinity of the upper wall.

The density profiles shown in Figure 4.14 indicate that up to a $30 \%$ and $45 \%$ increase in $\rho_{1}$ and $\rho_{2}$ respectively is attainable under uneven wetting conditions. Moreover, about a $35 \%$ maximum drop in both $\rho_{1}$ and $\rho_{2}$ is achievable under repulsive conditions. These variations in densities, compared to the even wetability case, indicates the use of uneven wetting conditions would increase separation efficiency. It should be noted that significant density variations occur within the hard core range

of intermolecular forces. In this case for separation purposes, channel outlets should be sized and located appropriately towards each wall.

A major difference between even and uneven wettabilities is the case of $N_{y}=15$. In Figure 4.12, the density remained below the initial value throughout the channel. 
In Figure 4.14 the density profile spans a range above and below the initial density. This indicates that the density deviation in Figure 4.12 for $N_{y}=15$ is indeed due to fluid concentration within the computationally hidden half step along the walls. In Figure 4.14 fluid particles are allowed to exist abundantly close to the center of the channel instead of being focused continuously to the walls into the computational half step of the wall.

From all the density profiles in this sections and the previous section, it is evident that the denser fluid, fluid-1 in our case, is more sensitive to repulsion forces than the less dense fluid, fluid-2. Contrarily, the attraction forces are more influential on the less dense fluid. This observation is consistent with the findings of Falcucci et al [66] based on the mathematical interpretation of the pseudo-potential force with the differential formulation of the Shan-Chen force

$$
F=-(1-\psi) \nabla \rho
$$

The interaction force becomes stronger as $\psi$ approaches zero, fluid is less dense. This corresponds to the decrease in the density at the central node where the force is calculated, considering the attraction case. Similarly, it is reasonable to say that repulsion effect is stronger on the heavier fluid. That requires $\psi$ to be greater than 1.0 for the denser fluid. 


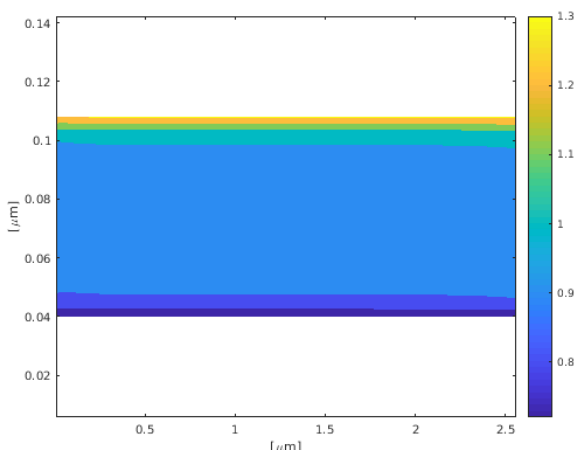

(a)

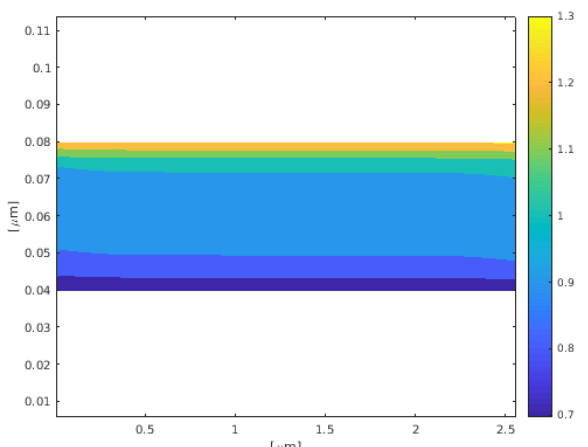

(c)

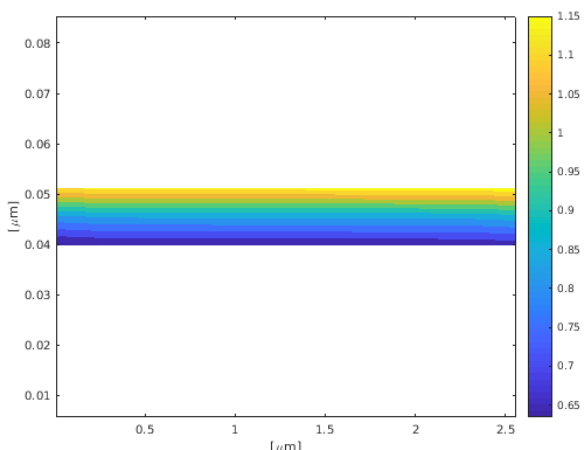

(e)

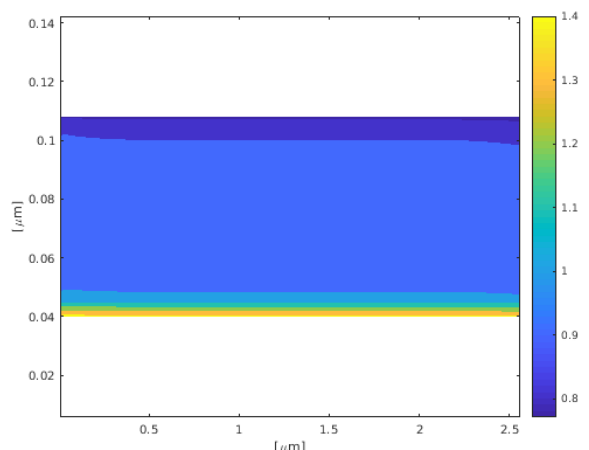

(b)

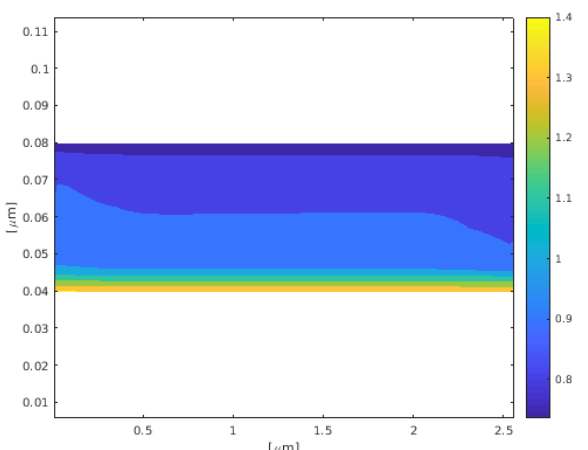

(d)

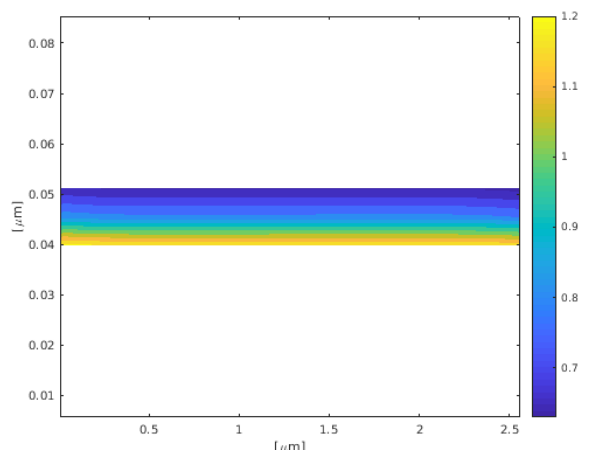

(f)

Figure 4.13: Scaled densities of both fluids ( $\rho_{1}$ left and $\rho_{2}$ right) at different channel heights under uneven wetting conditions where top has: $G_{1 s}=-1.0$ and $G_{2 s}=1.0$ and bottom: $G_{1 s}=1.0$ and $G_{2 s}=-1.0$ with fixed $\tau_{1}=\tau_{2}=0.75, G_{12}=0.5$. 


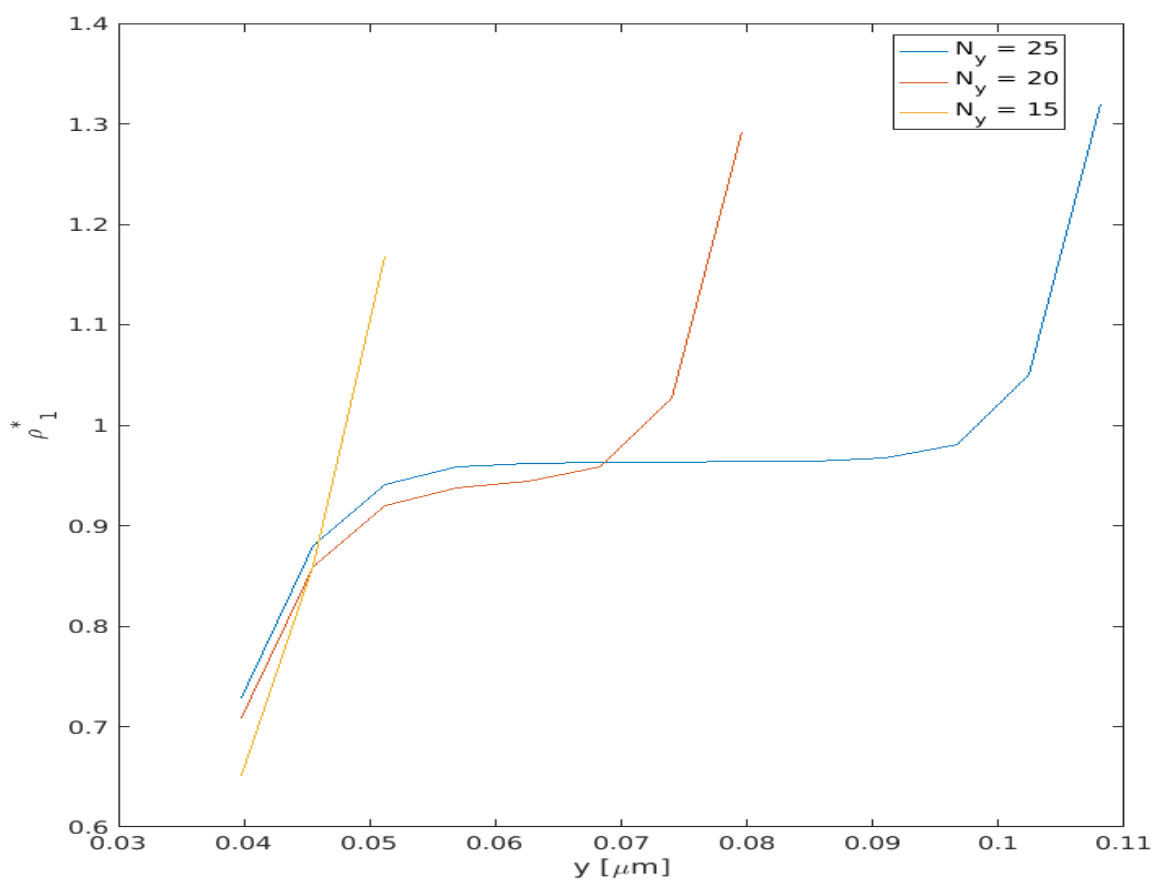

(a)

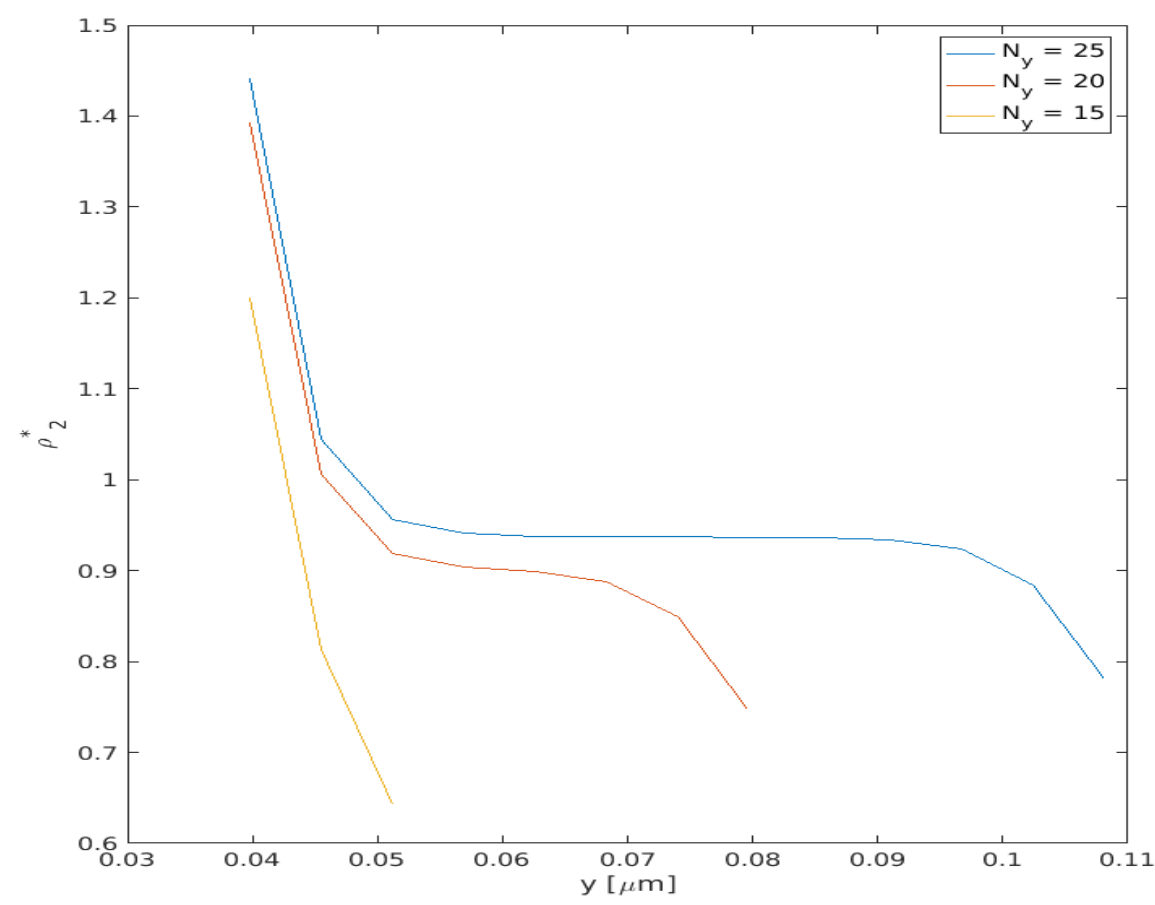

(b)

Figure 4.14: Profiles of scaled densities of both fluids $\left((\mathrm{a})-\rho_{1}^{*}=\frac{r h o_{1}}{r h o_{1}^{i n i t}}\right.$ and $(\mathrm{b})-\rho_{2}^{*}=$ $\left.\frac{\rho_{2}}{\rho_{2}^{\text {init }}}\right)$ at the middle of channel for heights under uneven wetting conditions where top has: $G_{1 s}=-1.0$ and $G_{2 s}=1.0$ and bottom: $G_{1 s}=1.0$ and $G_{2 s}=-1.0$ with fixed $\tau_{1}=\tau_{2}=0.75, G_{12}=0.5$. 


\subsection{Solid Posts Enhancement}

For the range of surface forces applied in sections 4.4 and 4.5 , significant gradients in fluid concentrations are limited to regions $30 \mathrm{~nm}$ of the wall. This makes it practically difficult to employ these forces for separations in microscale sized channels (channels of outlets that are not nanoscale in dimension and located at the walls). To extend the span of the intermolecular surface interactions to larger length scales, we examine adding solid posts of wettabilities similar to that of the neighbor wall. Hereby, the solid posts located above the center line of the channel have the solid-fluid interaction coefficients of the upper wall while solid posts located below the center line have the solid-fluid interaction coefficients of the lower wall.

In the following sections we study microchannels patterned fully or partially with solid posts of prescribed wettability. The spacing $S_{x}$ and $S_{y}$ are used to identify the distribution of solid posts in the $\mathrm{x}$ and $\mathrm{y}$ directions respectively. A single post is assumed to occupy a single node, cell, or lattice.

\subsection{Static Case}

In this section we examine the effect of changing the densities of solid posts under static conditions. This is achieved by the varying the post spacing in either the spanwise or flow direction. Periodic boundary conditions between the inlet and outlet are applied without forcing to impose the no flow conditions.

The effect of changing $S_{x}$ on both fluid densities is shown in Figure 4.15 where the upper half of the channel has a higher affinity for fluid-1 and a lower affinity for fluid-2. The lower half of the channel has a higher affinity for fluid-2 and a lower

affinity for fluid-1. It is noticeable that each fluid component concentrates the region of higher affinity. Complete separation is not achieved as the miscibility condition 
(favorable interaction between fluid-1 and fluid-2) stabilizes a consider amount of each fluid in its hydrophobic half. Sudden changes are observed near the walls where the fluid particles experience continuous interactions and along the centerline where the fluid particles experience an inflection in the wetting conditions.

The evolution of density profiles, along the mid-line of the channel, with time is presented in Fig. 4.16 for the cases $S_{x}=\frac{1}{6}, \frac{1}{4}, \frac{1}{2}$ respectively. In the beginning, both fluid densities are equivalent to their initial value. The surface forces effect starts to redistribute each component afterwards. The surface of higher attraction starts to withdraw the preferred fluid from its neighborhood leading to a considerable drop in the density of that area. On the other hand, the surface of higher repulsion displaces the particles away leading to augmentation in the density of the adjacent region. Just across the center, there is a transition regime where the components experience a smooth increment in their densities between the lower and higher affinity posts. As time passes, the density profiles start to flatten between the center and the wall until reaching their steady state.

The steady state density profiles are displayed in Figures 4.17 and 4.18 to show the impact of changing $S_{x}$ and $S_{y}$ respectively. By fixing $S_{y}$ and increasing $S_{x}$ the transition between the two levels of density, represented by flat regimes, extends appreciably signaling an increase in the separation efficiency. If $S_{y}$ is modified while $S_{x}$ is constant wavy profiles develop due to the inequality of distances between the fluid nodes and the solid posts. 


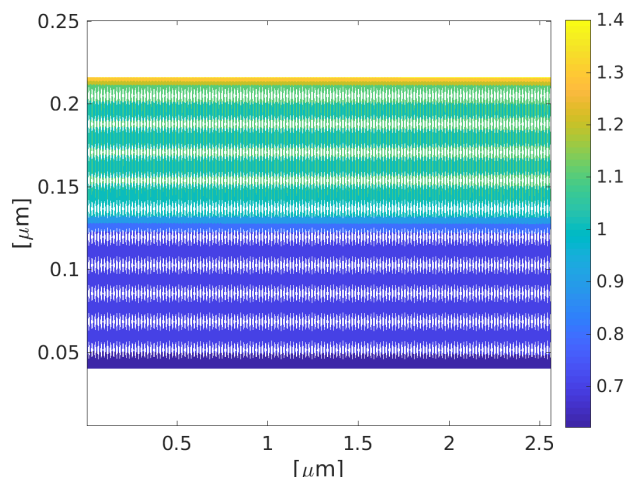

(a) $S_{x}=\frac{1}{6}$

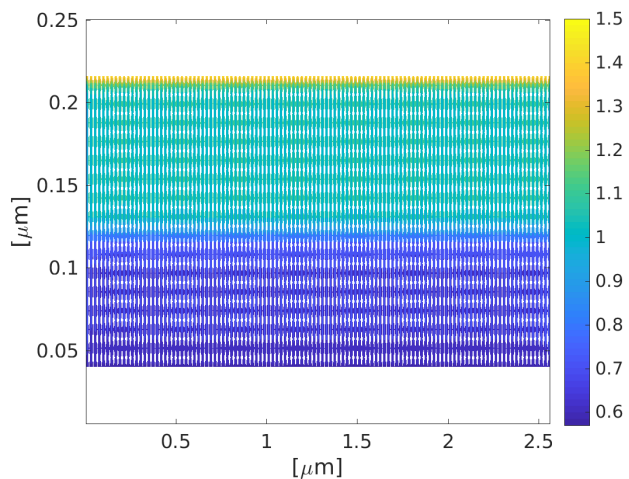

(c) $S_{x}=\frac{1}{4}$

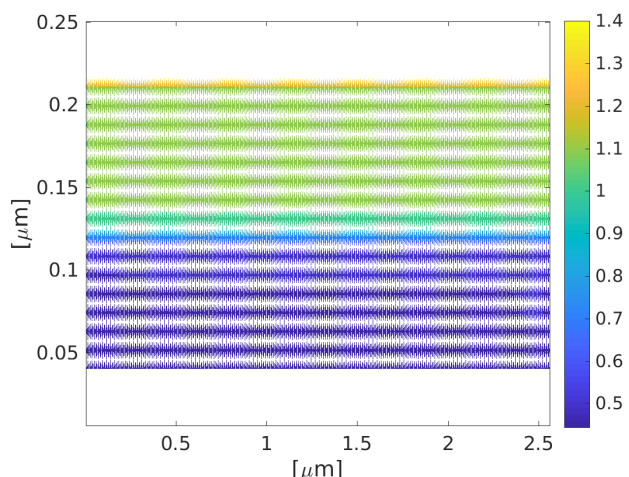

(e) $S_{x}=\frac{1}{2}$

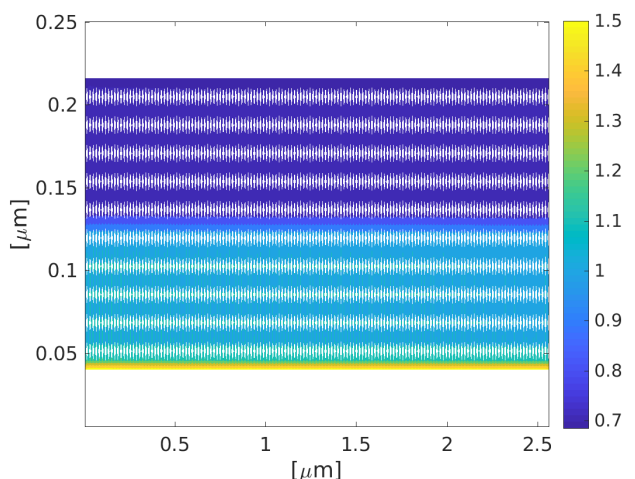

(b) $S_{x}=\frac{1}{6}$

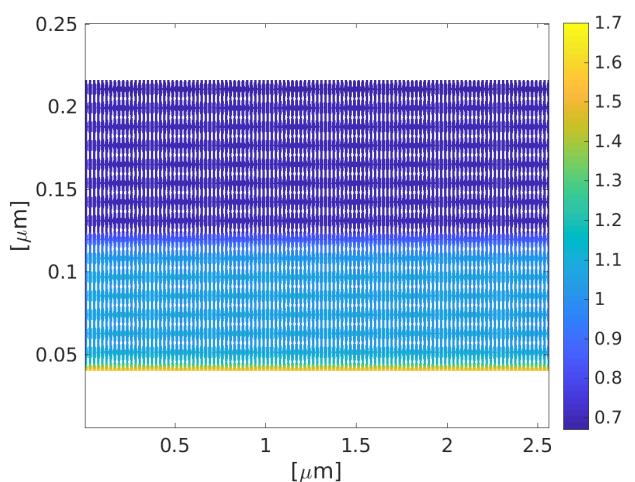

(d) $S_{x}=\frac{1}{4}$

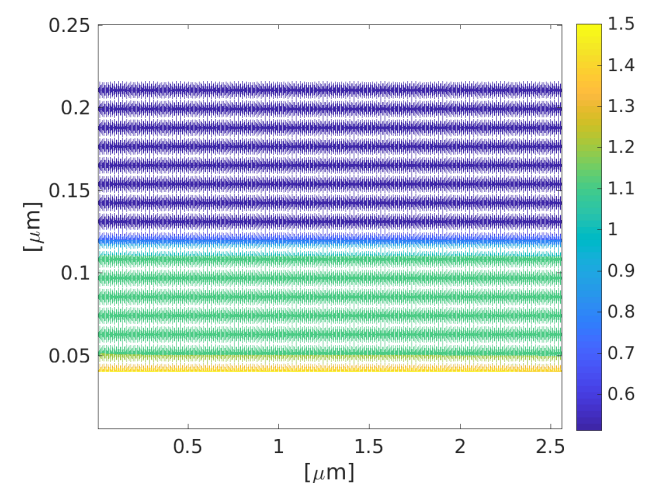

(f) $S_{x}=\frac{1}{2}$

Figure 4.15: Contours of scaled densities ( $\rho_{1}$ left and $\rho_{2}$ right) for variable horizontal spacing $S_{x}=\frac{1}{6}, \frac{1}{4}, \frac{1}{2}$ and $S_{y}=\frac{1}{2}$ with uneven wetting conditions where top has: $G_{1 s}=$ -1.0 and $G_{2 s}=1.0$ and bottom: $G_{1 s}=1.0$ and $G_{2 s}=-1.0$ with fixed $\tau_{1}=\tau_{2}=$ $0.75, G_{12}=1.0$. 


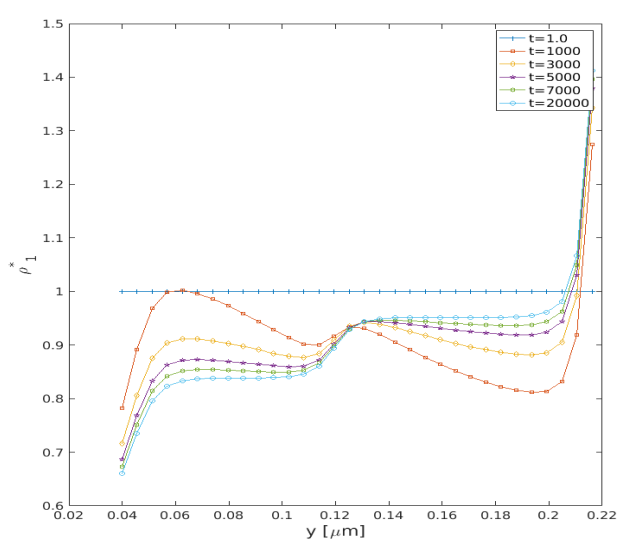

(a) $S_{x}=\frac{1}{6}$

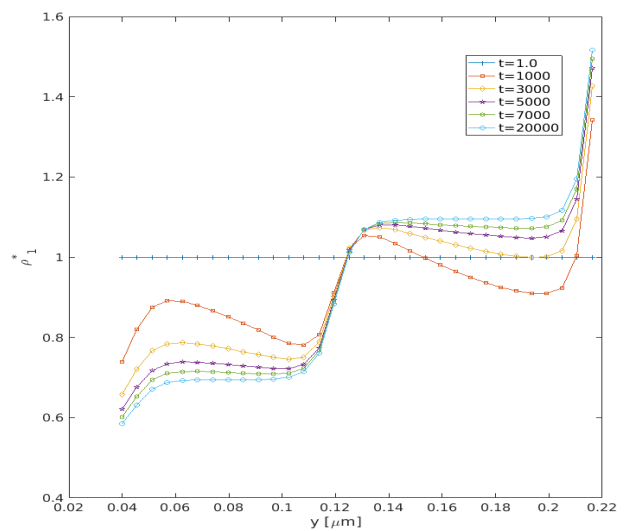

(c) $S_{x}=\frac{1}{4}$

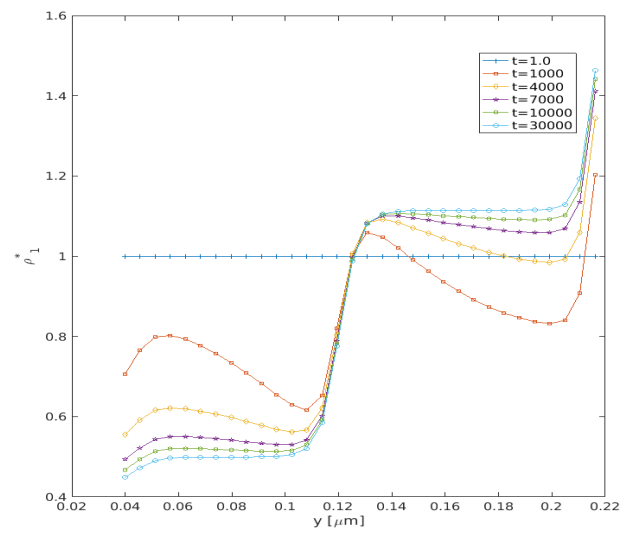

(e) $S_{x}=\frac{1}{2}$

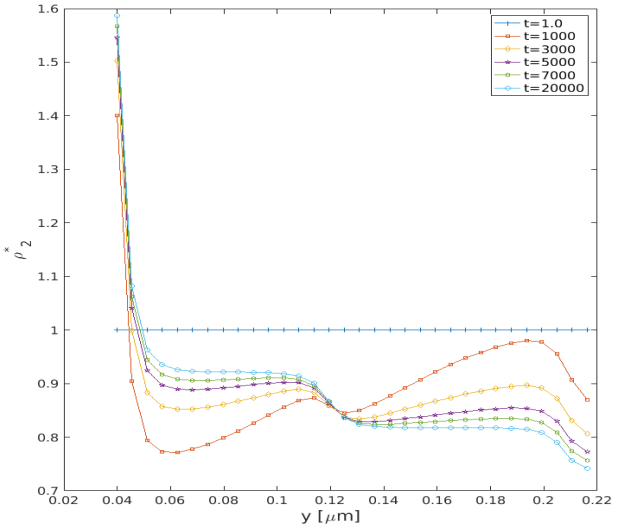

(b) $S_{x}=\frac{1}{6}$

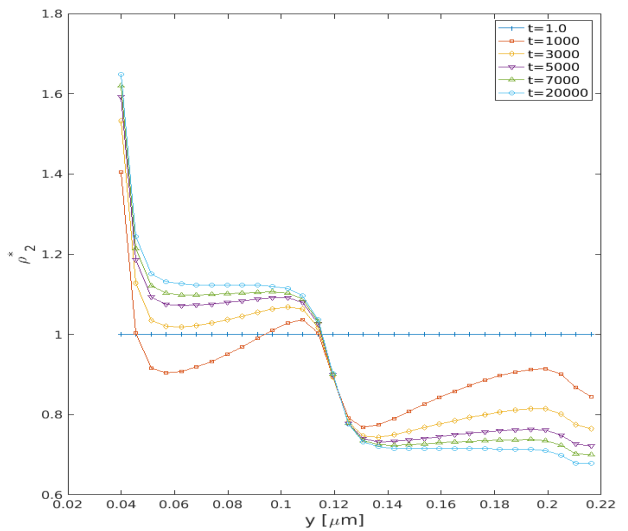

(d) $S_{x}=\frac{1}{4}$

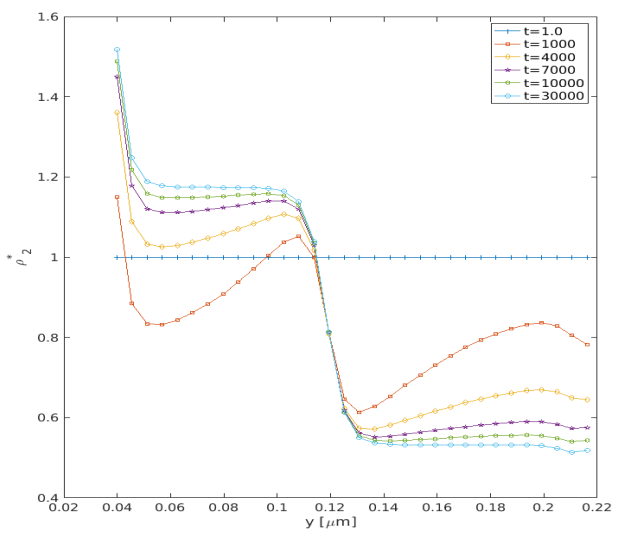

(f) $S_{x}=\frac{1}{2}$

Figure 4.16: Evolution with time of scaled densities profiles ( $\rho_{1}$ left and $\rho_{2}$ right) for variable horizontal spacing $S_{x}=\frac{1}{6}, \frac{1}{4}, \frac{1}{2}$ and $S_{y}=\frac{1}{2}$ with uneven wetting conditions where top has: $G_{1 s}=-1.0$ and $G_{2 s}=1.0$ and bottom: $G_{1 s}=1.0$ and $G_{2 s}=-1.0$ with fixed $\tau_{1}=\tau_{2}=0.75, G_{12}=1.0$. 


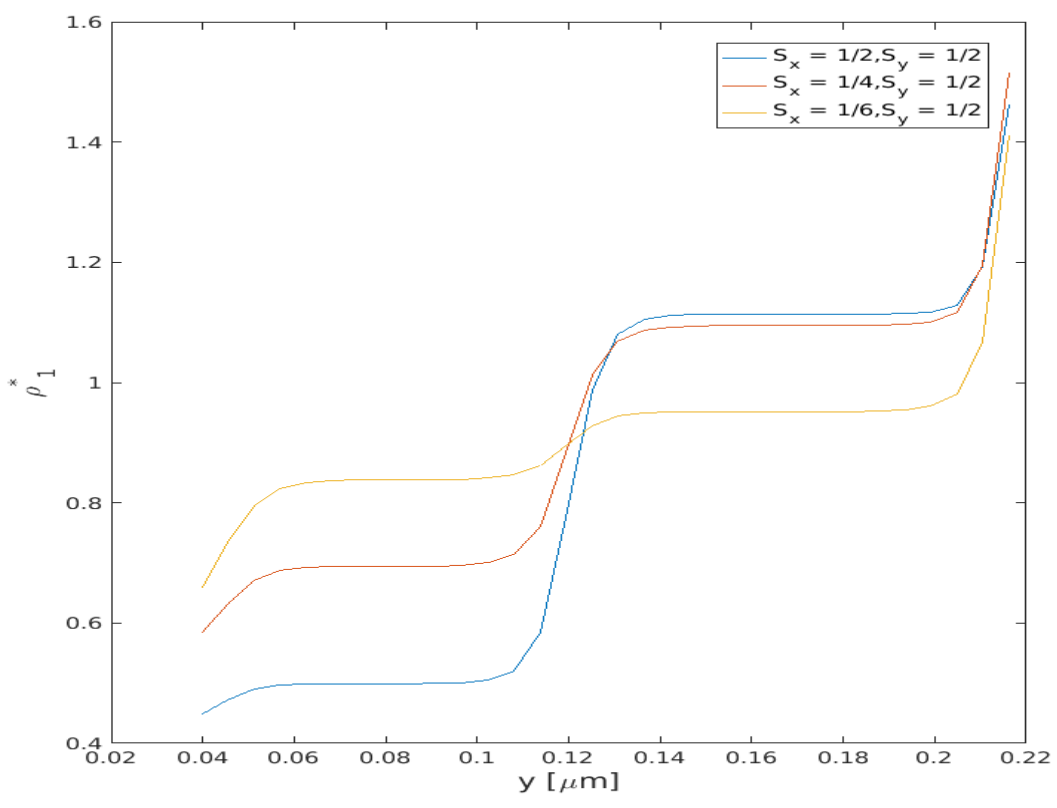

(a)

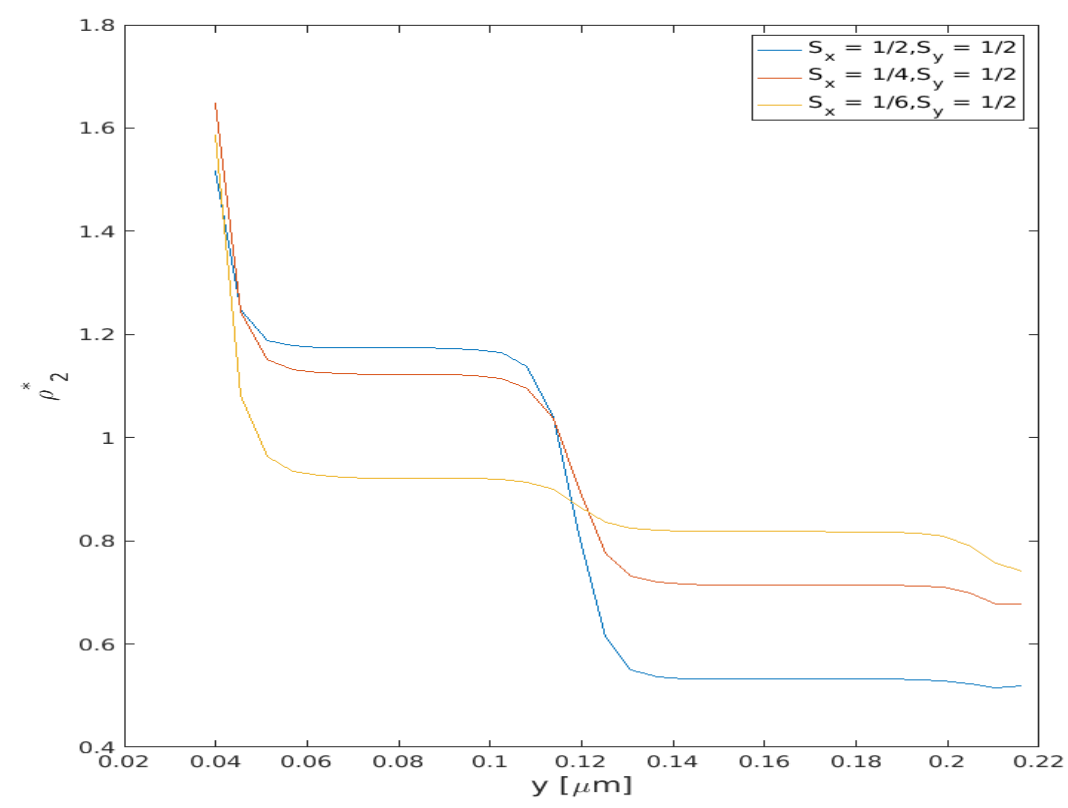

(b)

Figure 4.17: The steady state profiles of the scaled densities $\left((\mathrm{a})-\rho_{1}^{*}=\frac{r h o_{1}}{r h o_{1}^{i n i t}}\right.$ and (b) $\left.-\rho_{2}^{*}=\frac{\rho_{2}}{\rho_{2}^{i n i t}}\right)$ in a partially patterned channel for variable $S_{x}$ and fixed $S_{y}$ with uneven wetting conditions where top has: $G_{1 s}=-1.0$ and $G_{2 s}=1.0$ and bottom: $G_{1 s}=1.0$ and $G_{2 s}=-1.0$ with fixed $\tau_{1}=\tau_{2}=0.75, G_{12}=1.0$. Periodic inlet and outlet conditions are prescribed. 


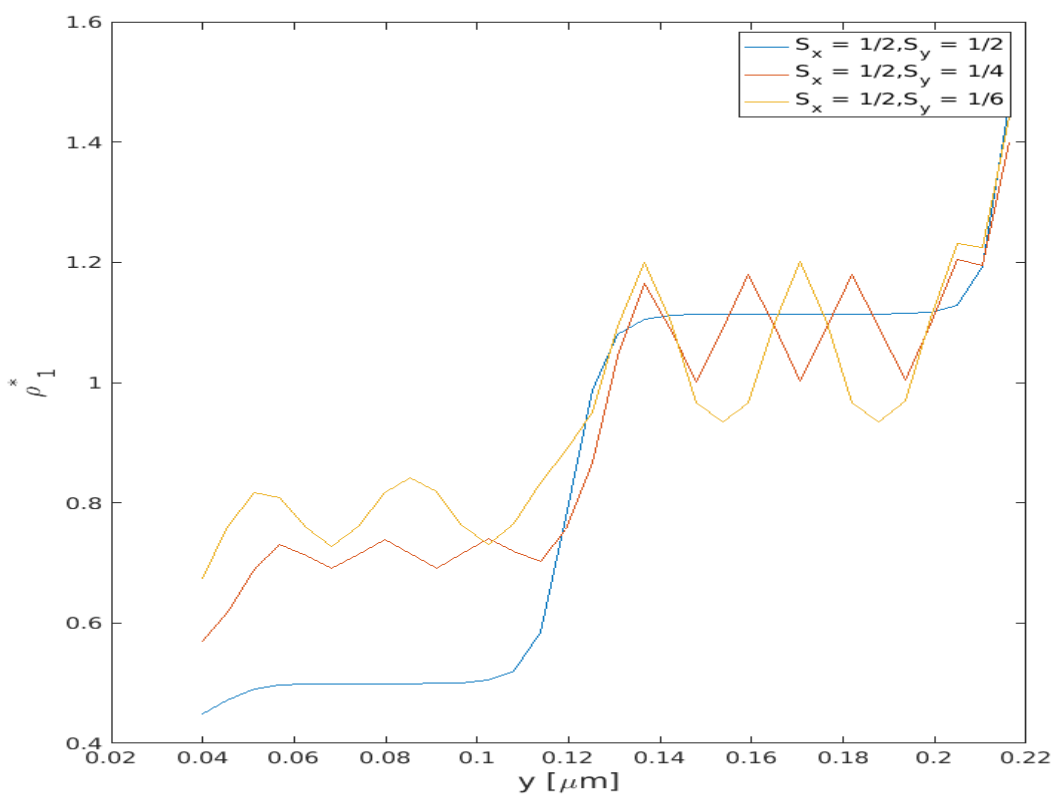

(a)

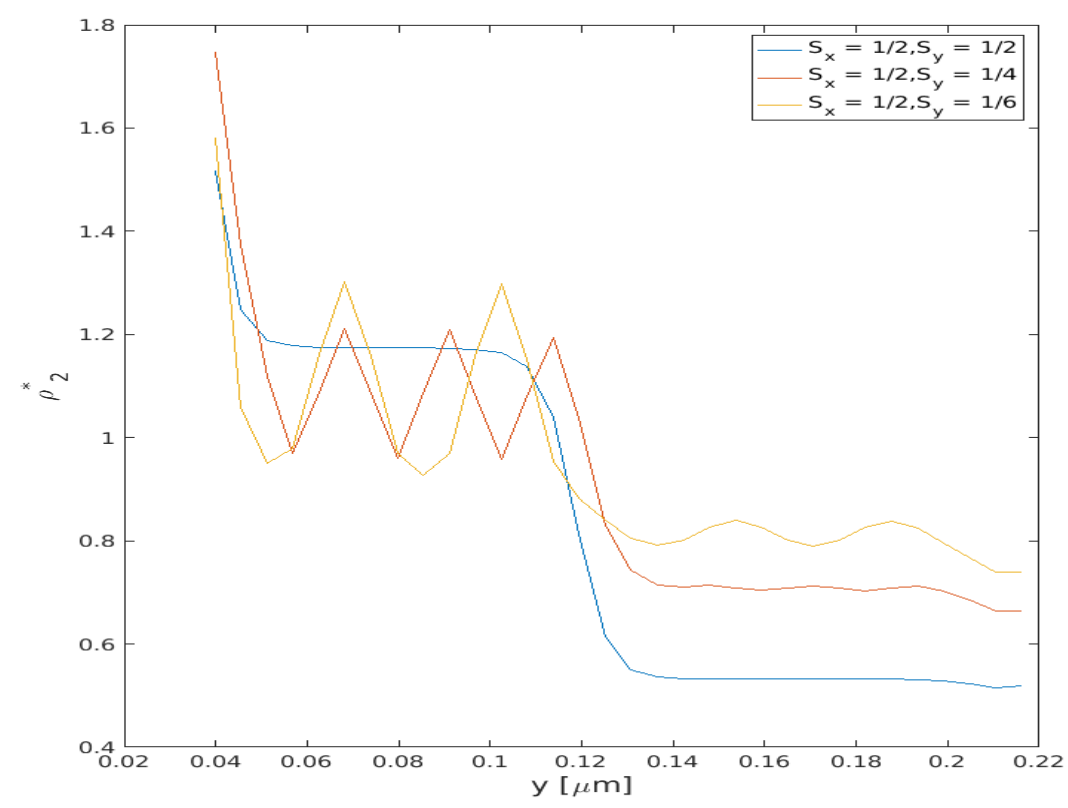

(b)

Figure 4.18: The steady state profiles of the scaled densities $\left((\mathrm{a})-\rho_{1}^{*}=\frac{r h o_{1}}{r h o_{1}^{i n i t}}\right.$ and (b) $\left.-\rho_{2}^{*}=\frac{\rho_{2}}{\rho_{2}^{i n i t}}\right)$ in a partially patterned channel for variable $S_{y}$ and fixed $S_{x}$ with uneven wetting conditions where top has: $G_{1 s}=-1.0$ and $G_{2 s}=1.0$ and bottom: $G_{1 s}=1.0$ and $G_{2 s}=-1.0$ with fixed $\tau_{1}=\tau_{2}=0.75, G_{12}=1.0$. Periodic inlet and outlet conditions are prescribed. 


\subsection{Dynamic Case}

While static profiles provide insight into potential separations, more practical interests include flow conditions. A partially patterned channel is used for this purpose where the solid posts start beyond the first quarter and ends at the third quarter of the channel. By using such configuration, the evolution of density profiles between pure fluid and the patterned region is straightforward to capture. There are many ways to induce flow using LBM depending on the boundary conditions prescription. Here we implemented two schemes to keep the inlet densities and the concentrations in sequence unaffected. The first one is the addition of an additional force, see Eq. 3.30 and Eq. 3.31, to the $\mathrm{x}$-component of the forcing term which is equivalent to a pressure drop locally. The periodic boundary condition between the inlet and outlet is unaffected and employed as the static case. The second scheme is to prescribe the inlet densities or the concentrations and the outlet velocity. The latter strategy does not entail any additional force to Shan-Chen forcing term.

The contour plot of densities when flow due to local forcing is imposed from left to right is shown in Figure 4.19. There are two distinguished spots in each plot where the densities reach their maximum or minimum values. The highest values are located in the beginning of the patterned region, near the upper wall for $\rho_{1}$ and near the lower wall for $\rho_{2}$. Conversely, the lowest are seen close to the lower wall for $\rho_{1}$ and close to the upper wall for $\rho_{2}$, at the end of the solid posts regime. The concentrated regions are not sustainable over the entire length of the pattern; they lose their intensity as one moves downstream.

The density profiles across and along the channel for the local forcing case are shown in Figure 4.20. The figures (a) and (b) display $\rho_{1}$ and $\rho_{2}$ profiles across the patterned section at two different times. Although they are shifted from each other 
due to the pressure difference, all these profiles follow the same trend as the static case. In Figure 4.20 (c) and Figure 4.20(d), the density evolution along the channel is presented at different heights. At a specific height, it is visible that the inlet and outlet densities are close to each other, an attribute that does not contradict the incompressiblity conditions. In spite of that, the profiles inside the channel indicate an aberration which alludes a nonphysical effect. This deviation is caused by the hydraulic resistance of the solid posts that slows the fluid motion inside the patterned section leading to pressure increase and decrease on both sides of that section.

Figure 4.21 shows the density contours when the inlet constant densities and outlet constant velocity conditions are prescribed. The inlet densities are fixed at $\rho_{1}=1.0$, and $\rho_{2}=0.78$ while the inlet velocity is left to the LBM to calculate. At the outlet the velocity is kept constant $\left(u_{i n}=1.0 \times 10^{-3}\right)$ while the densities are coupled by the LBM.The density distribution is apparently controlled by the wetting condition and its borders are more decent. The islands appearing near the inlet and outlet of the channel are due to shift in the interaction forces as they are deactivated around the areas where the flow boundary conditions are prescribed. In these regions, the wetting conditions are neutral.

The development of densities along the channel, specifically over the transition region between the pure fluid and the solid posts, points to a physically reasonable behavior as seen from Figure 4.22. It is evident from the profiles along $y / H=\frac{1}{4}, \frac{1}{2}$, and $\frac{3}{4}$ that densities faraway from the posts are very close to the inlet conditions. As one approaches the patterned section, densities either decrease or increase smoothly depending on the solid object wettability.

The dimensionless flow velocity profile in Figure 4.23 evolves as we move downstream under the viscous effect. The first and the last curves, $x / L=\frac{1}{6}$ and $x / L=\frac{5}{6}$, represent the velocity before and after the patterned area, which spans between 
$x / L=\frac{1}{4}$ and $x / L=\frac{3}{4}$, while the discrete lines are related to locations inside the patterning. As for any viscous flow in a straight channel, the velocity profile looks more flattened near the inlet and tends to be more parabolic downstream until being fully developed. Between the solid posts, however, the effective area of flow is smaller than it is in the pure fluid region; therefore, we see the average velocity is greater than its value outside the posts. A noticeable feature of all the profiles we have to mention is the lack of symmetry due to the barycentric approach that adopt a density weighted velocity.

Fig. 4.24 explains how the pressure drop between the inlet and outlet as well as the flow rate of the whole mixture are affected by altering the number of solid posts columns. The pressure drop acts in an opposite manner of the flow rate when the number of columns is increased. The nonlinear increase of the pressure drop as we add more solid posts is ascribed to the increase in hydraulic resistance of the channel.

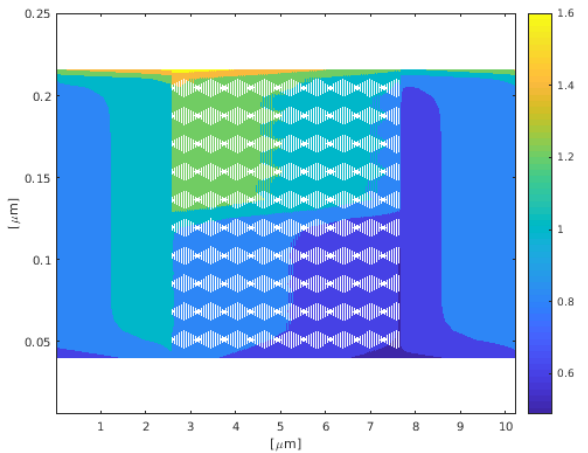

(a)

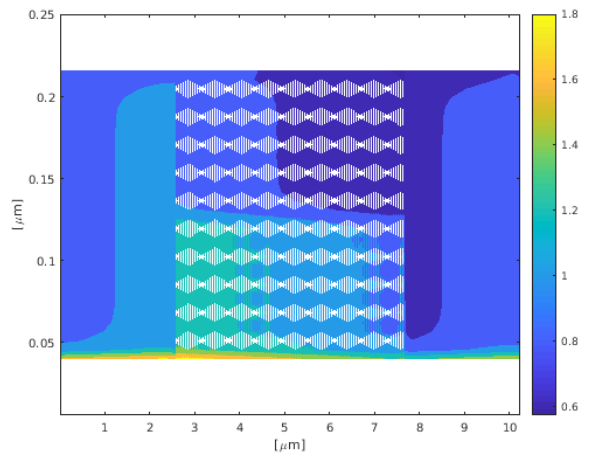

(b)

Figure 4.19: Contours of scaled densities ( $\rho_{1}$ left and $\rho_{2}$ right) in a partially patterned channel with fixed spacing $S_{x}=\frac{1}{3}$ and $S_{y}=\frac{1}{3}$ under uneven wetting conditions where top has: $G_{1 s}=-1.0$ and $G_{2 s}=1.0$ and bottom: $G_{1 s}=1.0$ and $G_{2 s}=-1.0$ with fixed $\tau_{1}=\tau_{2}=0.75, G_{12}=1.0$. Periodic inlet and outlet conditions are prescribed and the forcing term is $F_{x}=5 \times 10^{-4}$. 


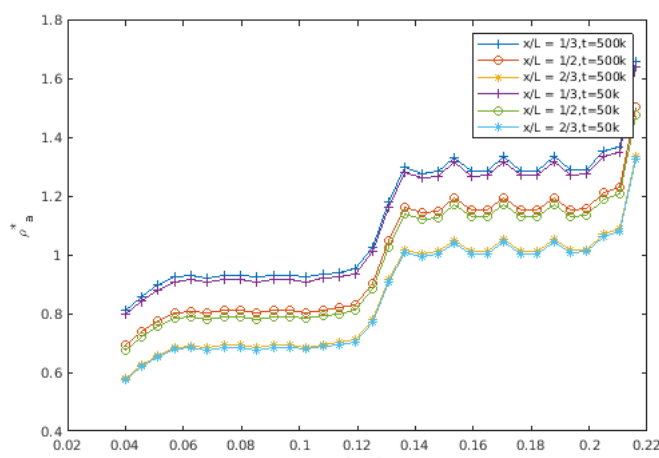

$(\mathrm{a})$

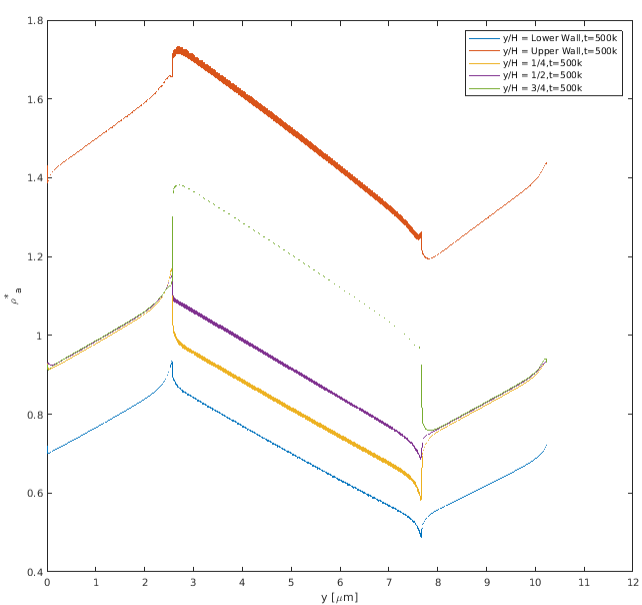

(c)

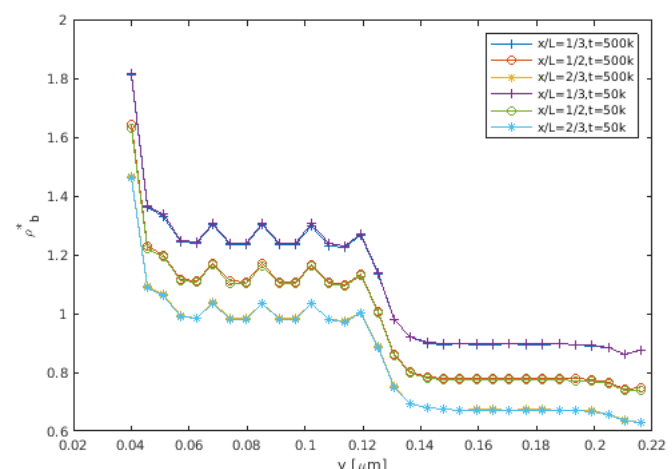

(b)

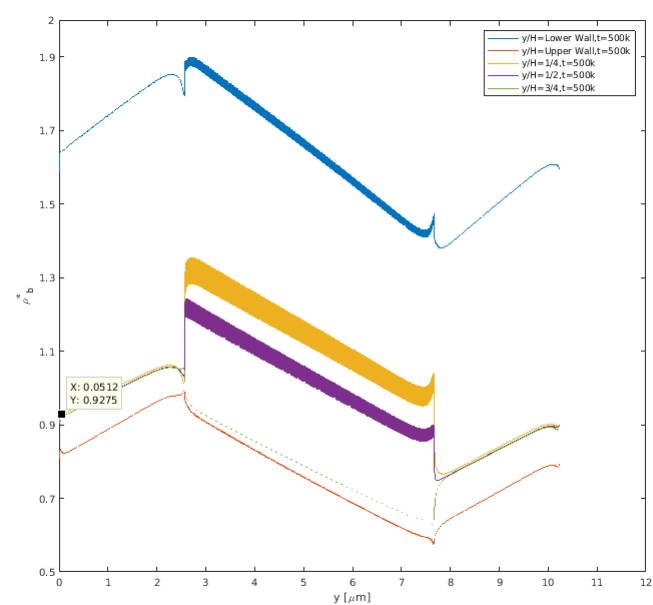

(d)

Figure 4.20: Scaled densities ( $\rho_{1}$ left and $\rho_{2}$ right) profiles across and along the channel in a partially patterned channel with fixed spacing $S_{x}=\frac{1}{3}$ and $S_{y}=\frac{1}{3}$ under uneven wetting conditions where top has: $G_{1 s}=-1.0$ and $G_{2 s}=1.0$ and bottom: $G_{1 s}=1.0$ and $G_{2 s}=-1.0$ with fixed $\tau_{1}=\tau_{2}=0.75, G_{12}=1.0$. Periodic Inlet and outlet conditions are prescribed and the forcing term is $F_{x}=5 \times 10^{-4}$. 


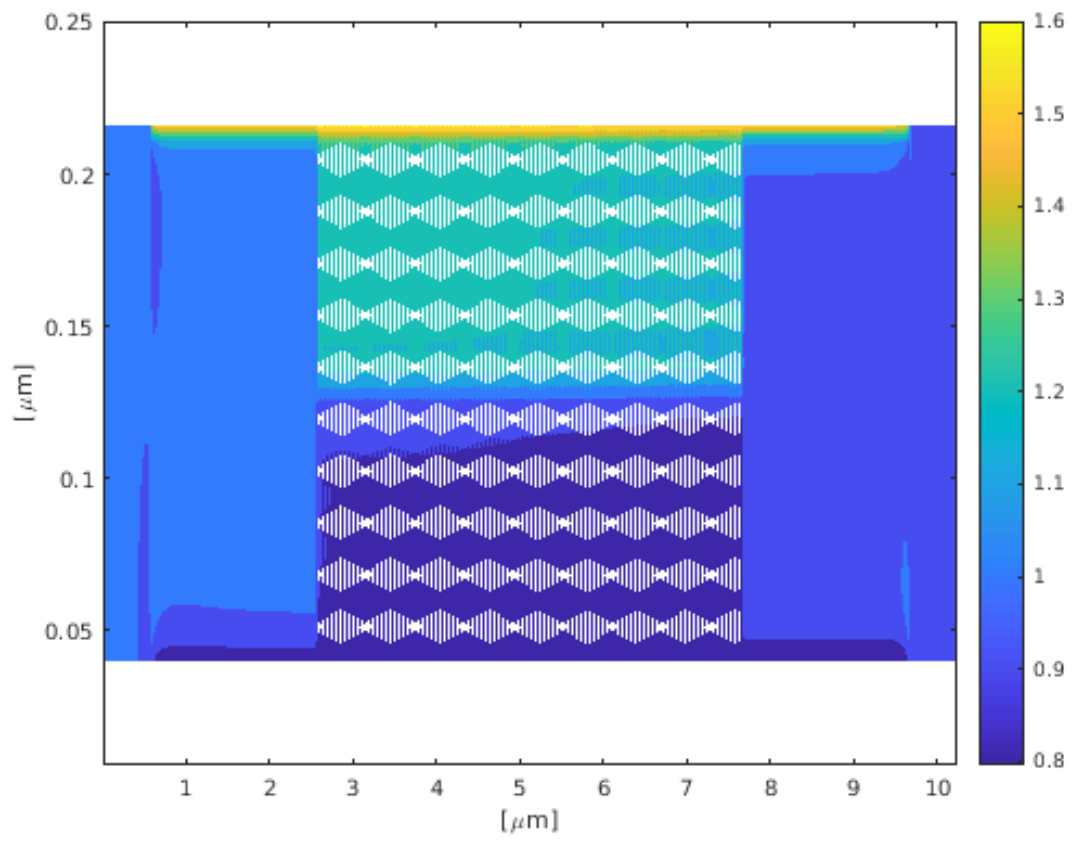

(a)

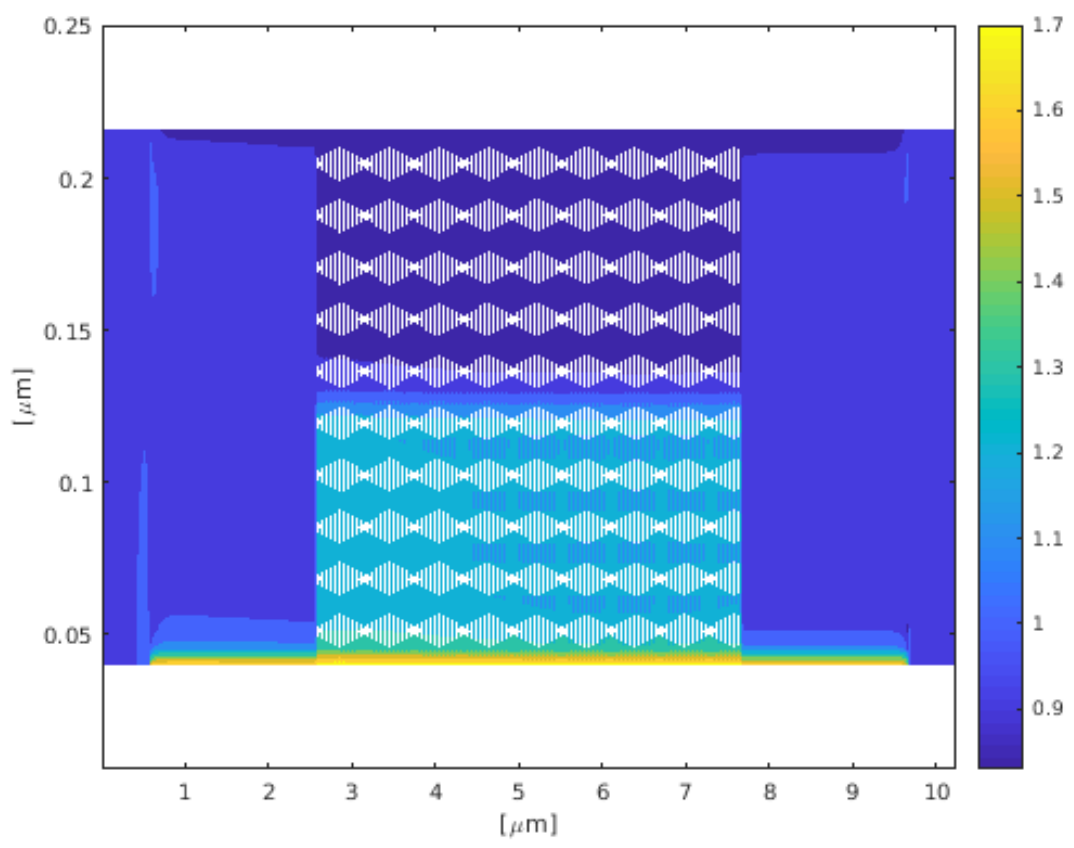

(b)

Figure 4.21: Contours of scaled densities ( $\rho_{1}$ left and $\rho_{2}$ right) in a partially patterned channel with fixed spacing $S_{x}=\frac{1}{3}$ and $S_{y}=\frac{1}{3}$ under uneven wetting conditions where top has: $G_{1 s}=-1.0$ and $G_{2 s}=1.0$ and bottom: $G_{1 s}=1.0$ and $G_{2 s}=-1.0$ with fixed $\tau_{1}=\tau_{2}=0.75, G_{12}=1.0$. Inlet densities and outlet velocity are Dirichlet. 


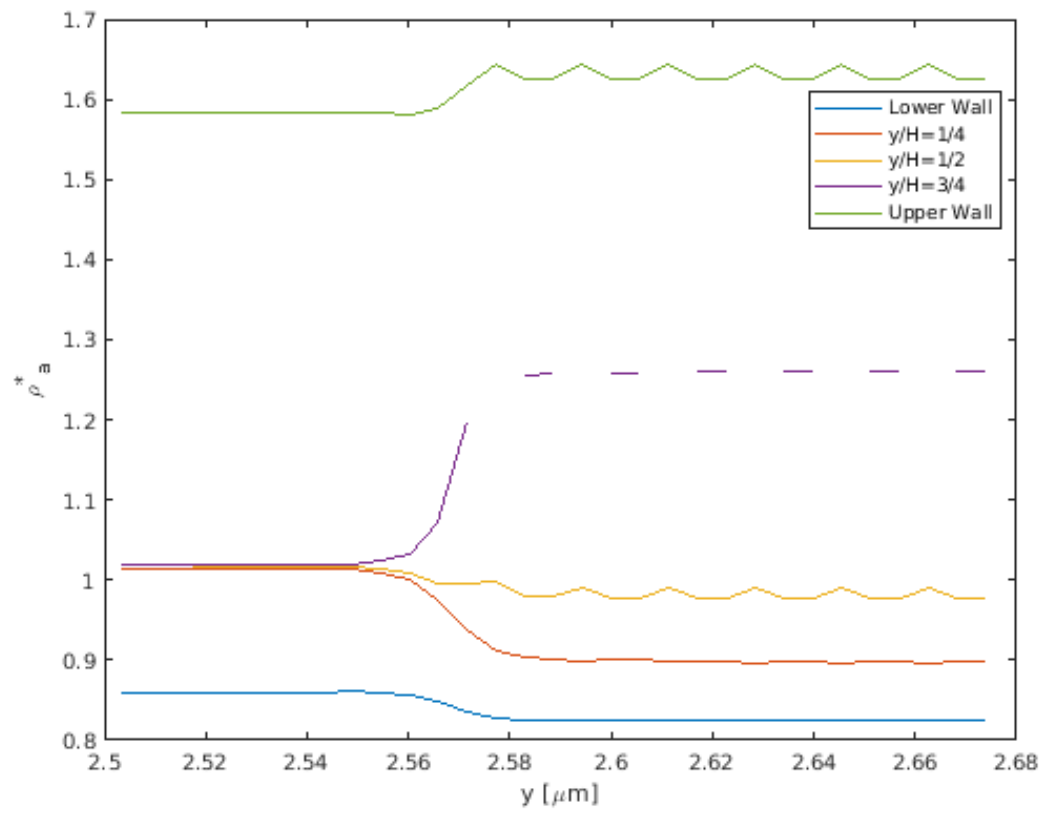

(a)

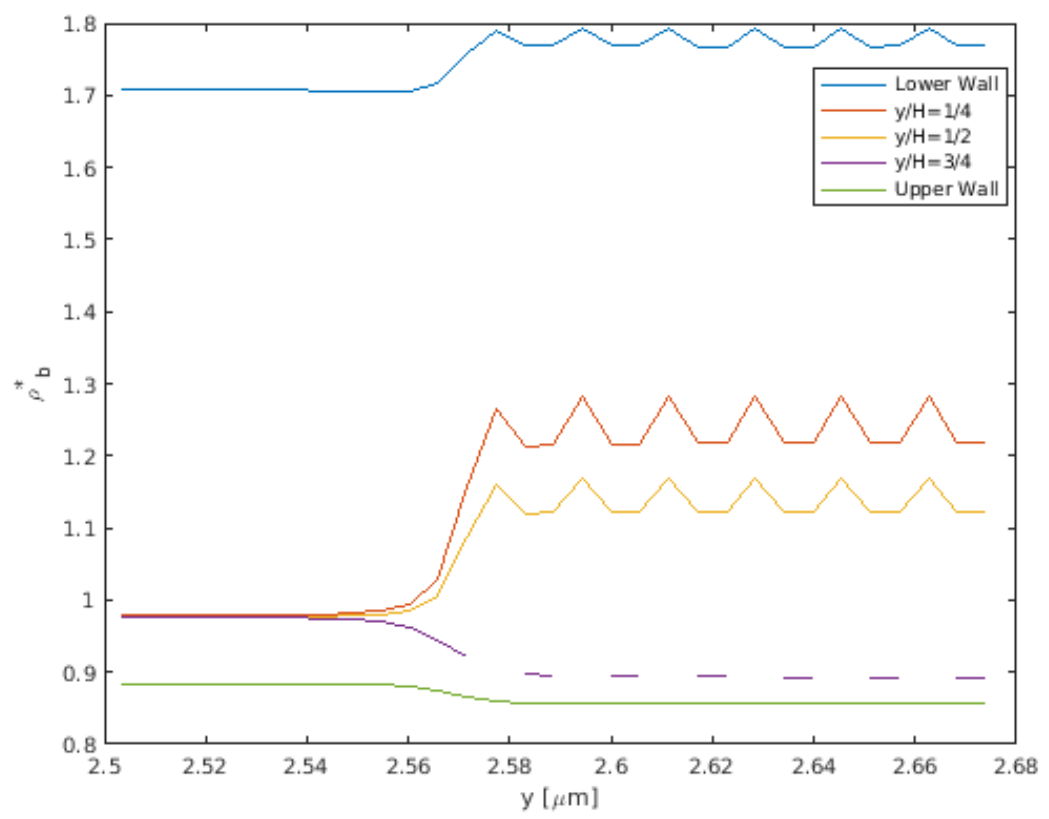

(b)

Figure 4.22: Scaled densities $\left((\mathrm{a})-\rho_{1}^{*}=\frac{r h o_{1}}{r h o_{1}^{i n i t}}\right.$ and $\left.(\mathrm{b})-\rho_{2}^{*}=\frac{\rho_{2}}{\rho_{2}^{i n i t}}\right)$ profiles along the channel in a partially patterned channel with fixed spacing $S_{x}=\frac{1}{3}$ and $S_{y}=\frac{1}{3}$ under uneven wetting conditions where top has: $G_{1 s}=-1.0$ and $G_{2 s}=1.0$ and bottom: $G_{1 s}=1.0$ and $G_{2 s}=-1.0$ with fixed $\tau_{1}=\tau_{2}=0.75, G_{12}=1.0$. Inlet densities and outlet velocity are Dirichlet. 


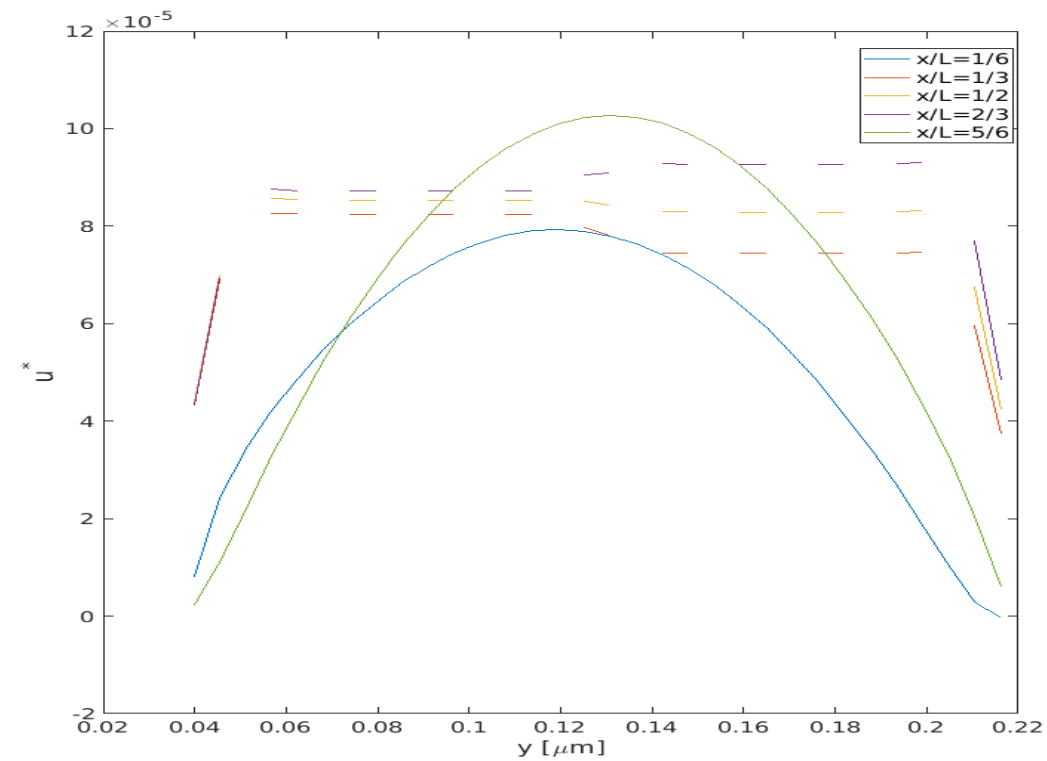

Figure 4.23: Dimensionless velocity profiles in a partially patterned channel with fixed spacing $S_{x}=\frac{1}{3}$ and $S_{y}=\frac{1}{3}$ under uneven wetting conditions where top has: $G_{1 s}=-1.0$ and $G_{2 s}=1.0$ and bottom: $G_{1 s}=1.0$ and $G_{2 s}=-1.0$ with fixed $\tau_{1}=$ $\tau_{2}=0.75, G_{12}=1.0$. Inlet densities and outlet velocity are Dirichlet.

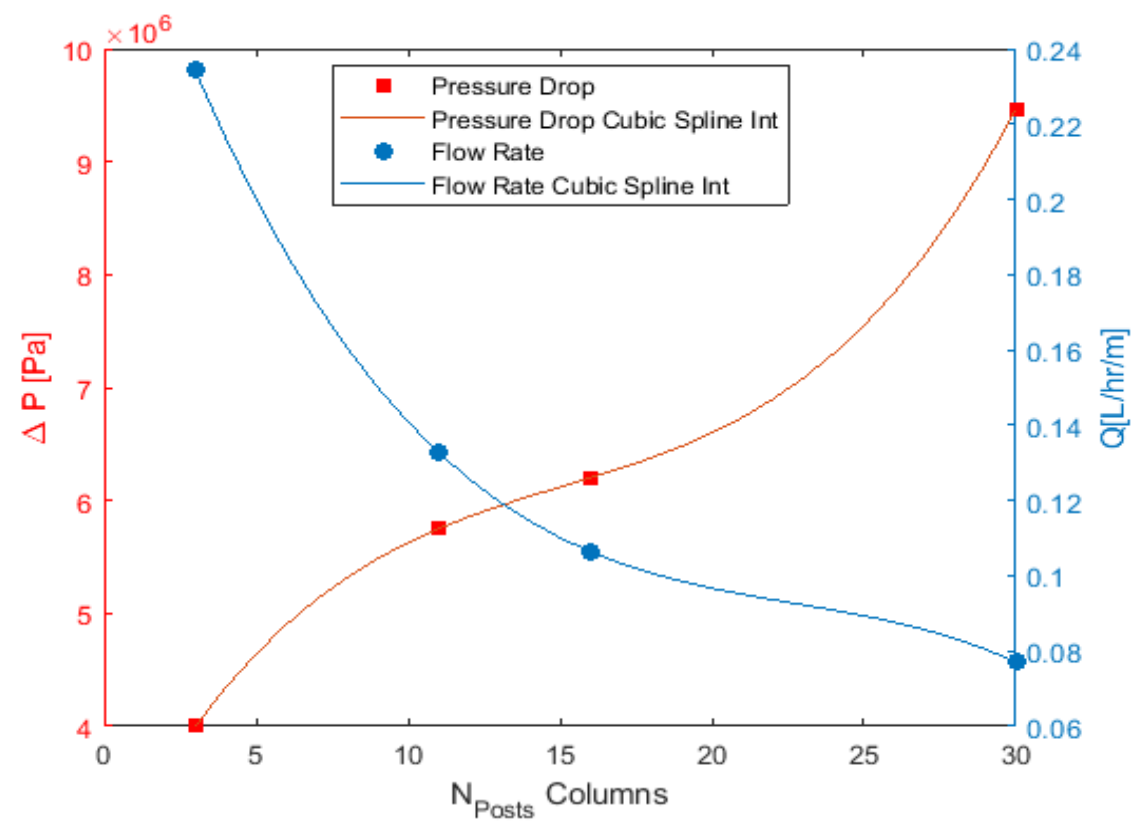

Figure 4.24: Number of solid posts columns on pressure gradient and flow rate at $S_{x}=\frac{1}{3}$ and $S_{y}=\frac{1}{3}$ under uneven wetting conditions where top has: $G_{1 s}=-1.0$ and $G_{2 s}=1.0$ and bottom: $G_{1 s}=1.0$ and $G_{2 s}=-1.0$ with fixed $\tau_{1}=\tau_{2}=0.75, G_{12}=1.0$. Inlet densities and outlet velocity are Dirichlet. 


\section{Chapter 5}

\section{Two-Phase Flow Separation}

This chapter focuses on the passive separation of two-phase flow in microchannels by surface forces. The D3Q27 LBM is implemented with the aid of multi-components Shan-Chen model to investigate the dynamical behavior of a droplet of one fluid driven by another fluid to a T-junction. The channel has variable wetting conditions where one half has a variable wettability while the other half has a fixed interaction coefficients. The fluids inside the channel are of different properties. The droplet density is fixed at $\rho_{1}=1.0$ (corresponding to $1000 \mathrm{Kg} / \mathrm{m}^{3}$ in physical units) and the continuous phase density is kept as $\rho_{2}=0.77\left(770 \mathrm{Kg} / \mathrm{m}^{3}\right.$ in physical units).

As stated in Sec. 3.4 both fluids are coupled by the interface which makes the selection of physical space step, $\Delta x^{p}$, uncomplicated. In addition, the appropriate

coupling between the spacial and temporal steps was found to be $\Delta t=\Delta x^{1.25}$, which is capable of retrieving the common values of surface tension and viscosities. Thus, choosing $\Delta x^{p}=1.0[\mu m]$ produces $\Delta t^{p}=31.622[n s]$ which leads to a surface tension $\sigma=44[\mathrm{mN} / \mathrm{m}]$. With relaxation times set at $\tau_{1}=\tau_{2}=1.5$ the kinematic viscosities inside and outside the droplet are $\nu_{1}=\nu_{2}=1.0 \times 10^{-5}\left[\mathrm{~m}^{2} / \mathrm{s}\right]$.

In the next sections we first study droplets evolution in static and dynamic flow conditions under uneven wettabilities; second we examine the effects of droplet initial length and the capillary number on drop breakup; and third we establish Ca-L phase 
diagrams to identify the breakup and non breakup regimes due to the change of wetting conditions, droplet to outer fluid viscosity ratio, the aspect ratio of the channel, and the side to main channel width ratio.

\subsection{Initialization Process}

Before moving to the dynamics of droplets under uneven wetting conditions first investigate how droplets evolve under static conditions when they are confined between walls of unequal contact angles. The contact angle of the lower half of the channel is fixed at $\theta_{\text {lower }}=173.2 \pm 7.1$, where as the upper half contact angle ranged as $\theta_{\text {upper }}=43.0 \pm 0.2$ to $143.2 \pm 0.8$. This is achieved by manipulating the solid-fluid interaction coefficients $G_{1 s}$ and $G_{2 s}$ as we detailed in Section 3.5. Figure 1.3 on page 6 provides the geometry of the channel. Table 5.1 provides the microscale dimensions of the T-channel setup.

In all the cases studied in this chapter, the droplet is initialized as cuboid of the same area as the main channel, $A=D \times W$, and of an initial length $L_{0}$. Then the simulation was run for $1.0 \times 10^{5}$ time steps to allow the droplet to develop its shape and final interfacial profile. Both the final droplet shape and the droplet size differ from their initial state. The droplet shape evolution is governed by the minimization of surface energy while the the droplet size is associated with the Shan-Chen model's definition of major and minor densities in the system. The total mass of the entire system is conserved.

Table 5.1: The dimensions of the T-junctions used for passive separation as Fig. 1.3 shows.

\begin{tabular}{|c|c|c|c|c|}
\hline W & $W_{1}$ & D & Main Channel Length & Side Channel Length \\
\hline $30[\mu \mathrm{m}]$ & $30[\mu \mathrm{m}]$ & $30[\mu \mathrm{m}]$ & $220[\mu \mathrm{m}]$ & $360[\mu \mathrm{m}]$ \\
\hline
\end{tabular}


Figure 5.1 to 5.7 show the density contours at the middle of the channel for the case $L_{0}=2 W$ and $\tau_{1}=\tau_{2}=1.5$. These results indicate as the difference between $\theta_{\text {lower }}$ and $\theta_{\text {upper }}$ increases the time for droplets to reach their steady state profiles increases. Moreover the droplet spreads to the higher wettability half (the upper one) which has a lower contact angle. Because of the limited size of the droplet, the area of contact between the lower wall and the droplets is reduced as the difference in contact angles increases. Eventually, the droplets loses contact with lower wall, as shown in Figure 5.5. However, there is still contact between the droplet and the lower half via the walls parallel to the page, the front and back walls. When the affinity between the droplet and the upper section dominates, the droplet will leave the lower section completely and settles at the top (see Figure 5.7). 

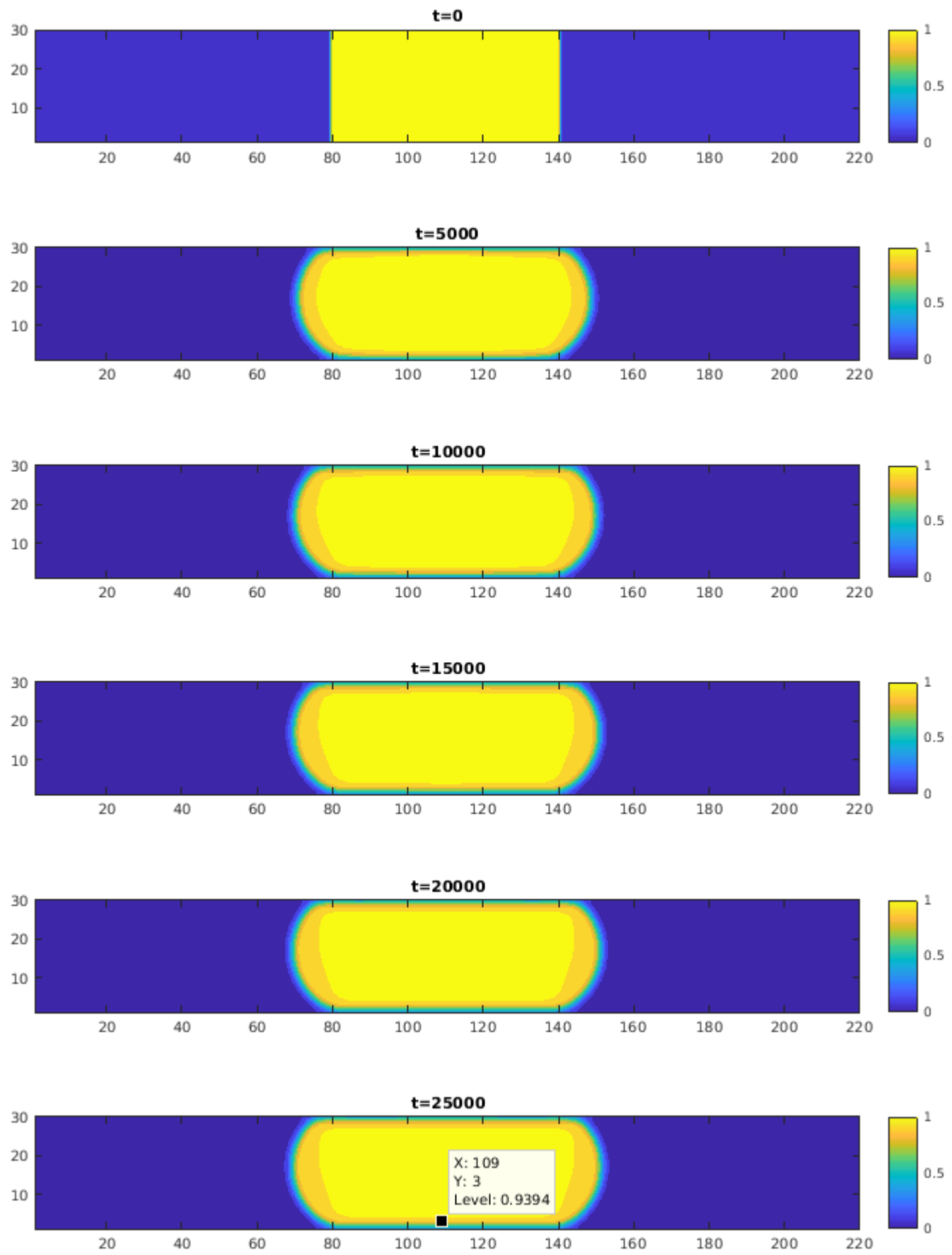

Figure 5.1: Initialization of a droplet of density $\rho_{1}=1.0$ and viscosity $\nu_{1}=0.33$ surrounded by a fluid of density $\rho_{2}=0.77$ and viscosity $\nu_{2}=0.33$ when $\Delta \theta=30$. Dimensions in $[\mu m]$ and each time step is $31.622[\mathrm{~ns}]$. 

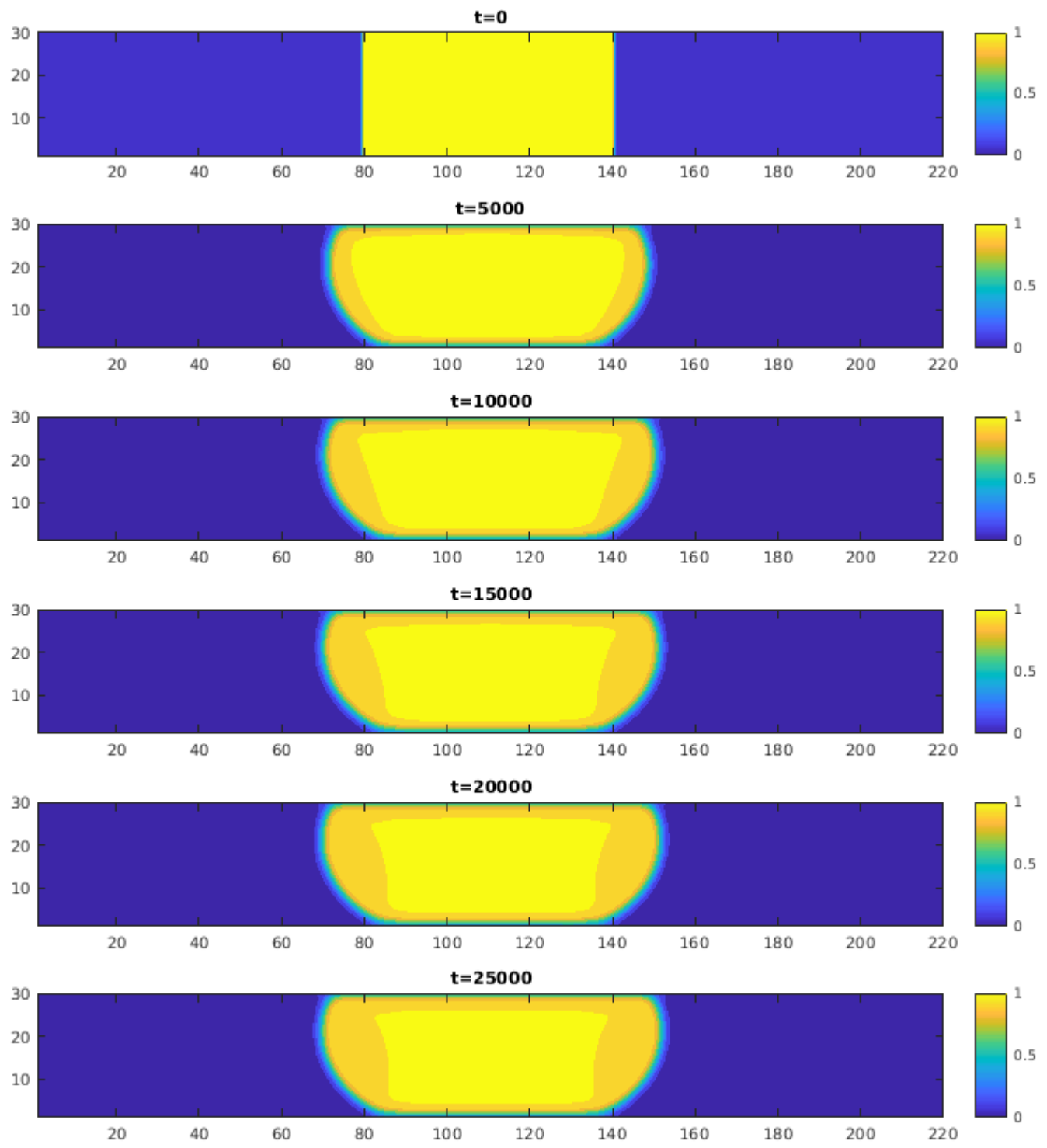

Figure 5.2: Initialization of a droplet of density $\rho_{1}=1.0$ and viscosity $\nu_{1}=0.33$ surrounded by a fluid of density $\rho_{2}=0.77$ and viscosity $\nu_{2}=0.33$ when $\Delta \theta=53$. Dimensions in $[\mu m]$ and each time step is $31.622[\mathrm{~ns}]$. 

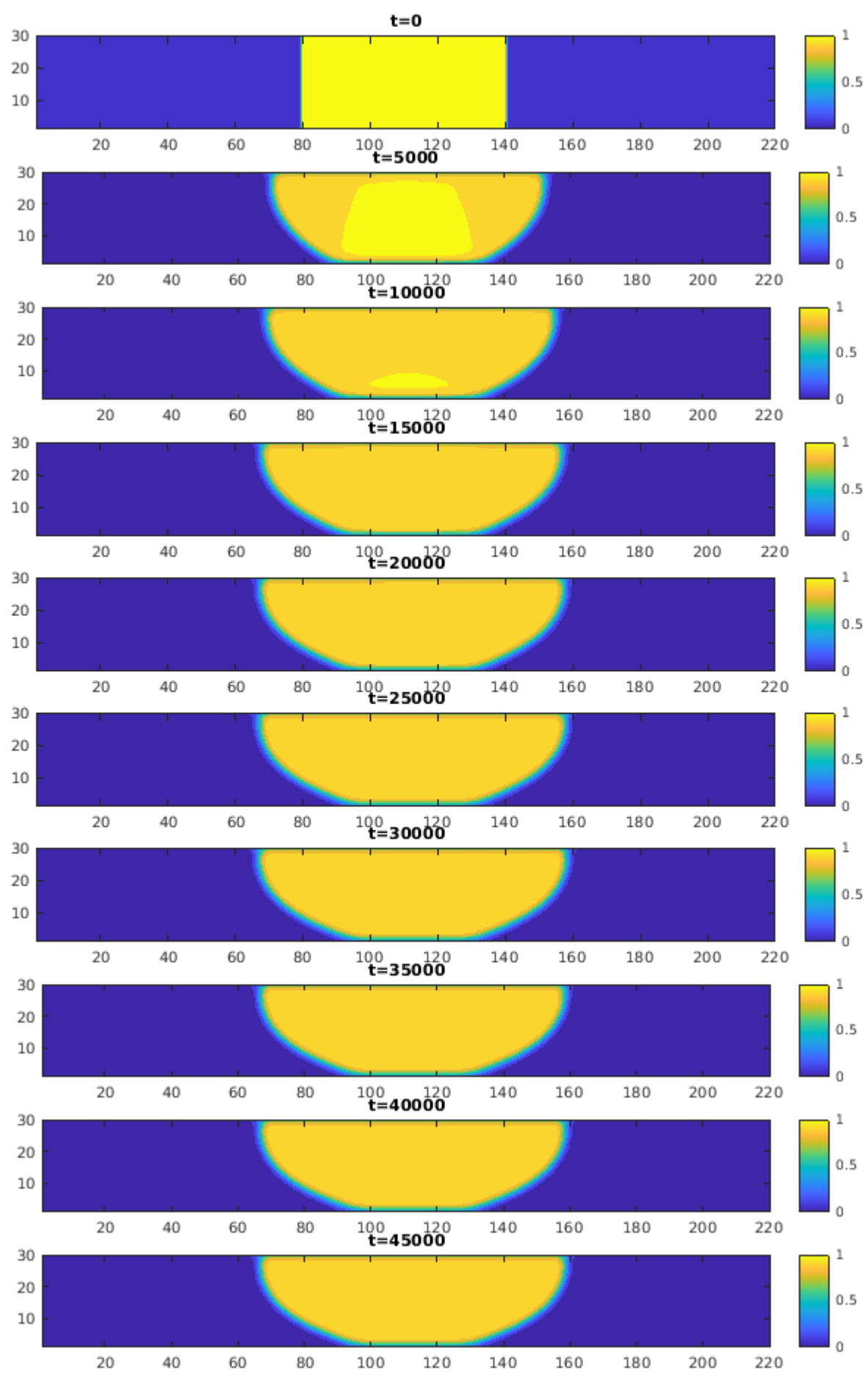

Figure 5.3: Initialization of a droplet of density $\rho_{1}=1.0$ and viscosity $\nu_{1}=0.33$ surrounded by a fluid of density $\rho_{2}=0.77$ and viscosity $\nu_{2}=0.33$ when $\Delta \theta=$ 70.Dimensions in $[\mu m]$ and each time step is $31.622[\mathrm{~ns}]$. 

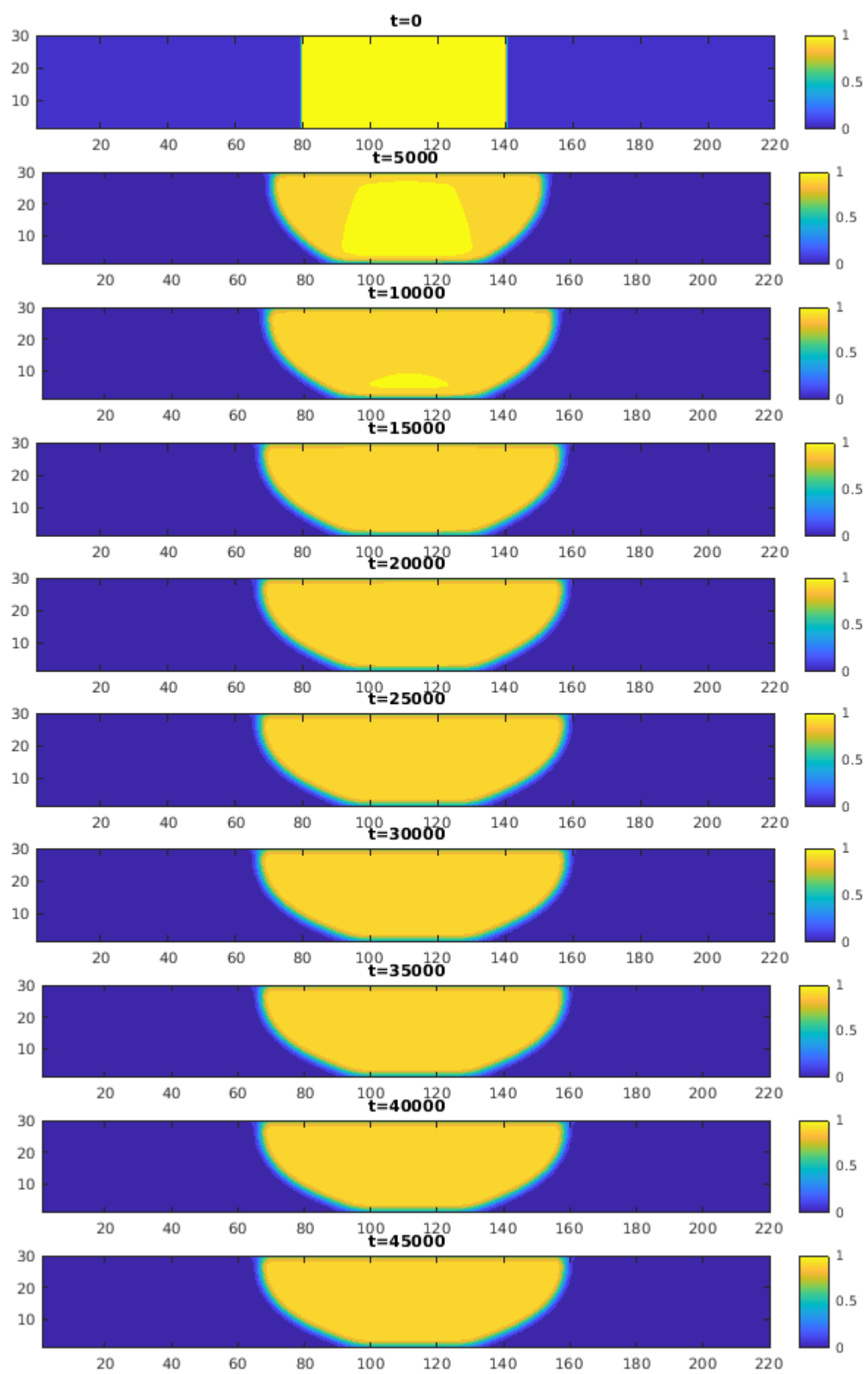

Figure 5.4: Initialization of a droplet of density $\rho_{1}=1.0$ and viscosity $\nu_{1}=0.33$ surrounded by a fluid of density $\rho_{2}=0.77$ and viscosity $\nu_{2}=0.33$ when $\Delta \theta=78$. Dimensions in $[\mu m]$ and each time step is $31.622[\mathrm{~ns}]$. 

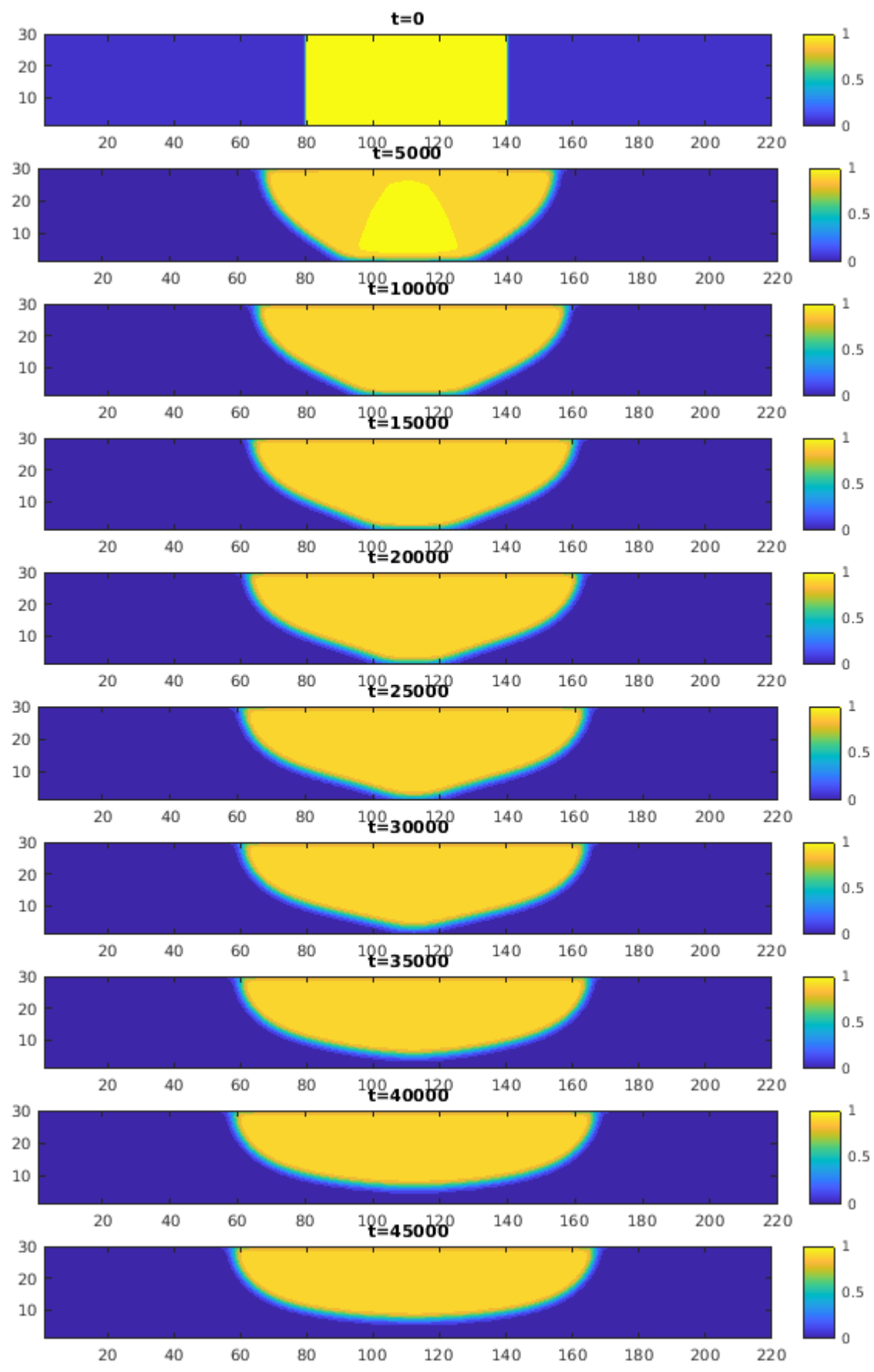

Figure 5.5: Initialization of a droplet of density $\rho_{1}=1.0$ and viscosity $\nu_{1}=0.33$ surrounded by a fluid of density $\rho_{2}=0.77$ and viscosity $\nu_{2}=0.33$ when $\Delta \theta=90$. Dimensions in $[\mu m]$ and each time step is $31.622[\mathrm{~ns}]$. 

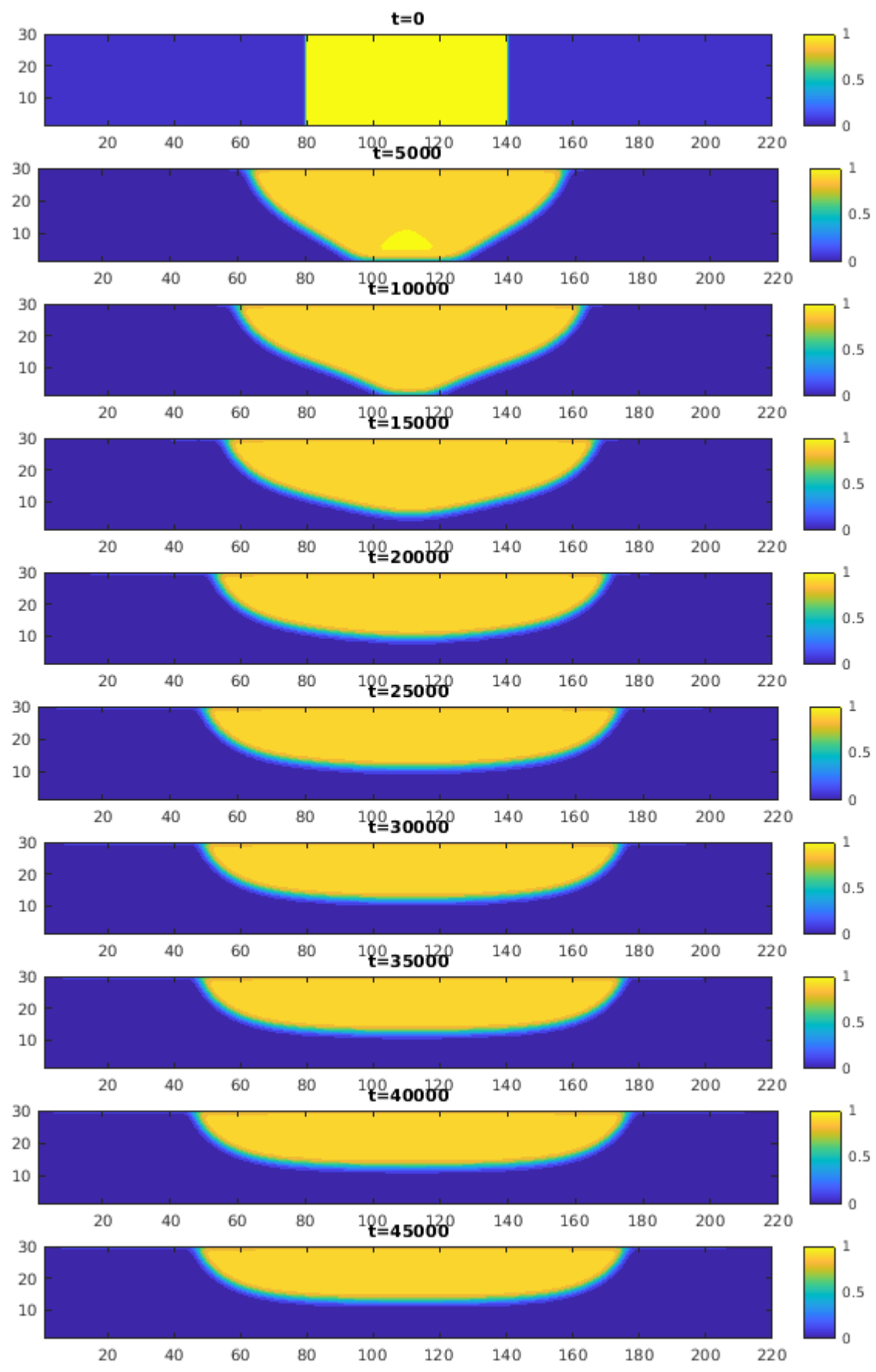

Figure 5.6: Initialization of a droplet of density $\rho_{1}=1.0$ and viscosity $\nu_{1}=0.33$ surrounded by a fluid of density $\rho_{2}=0.77$ and viscosity $\nu_{2}=0.33$ when $\Delta \theta=104$. Dimensions in $[\mu m]$ and each time step is $31.622[\mathrm{~ns}]$. 

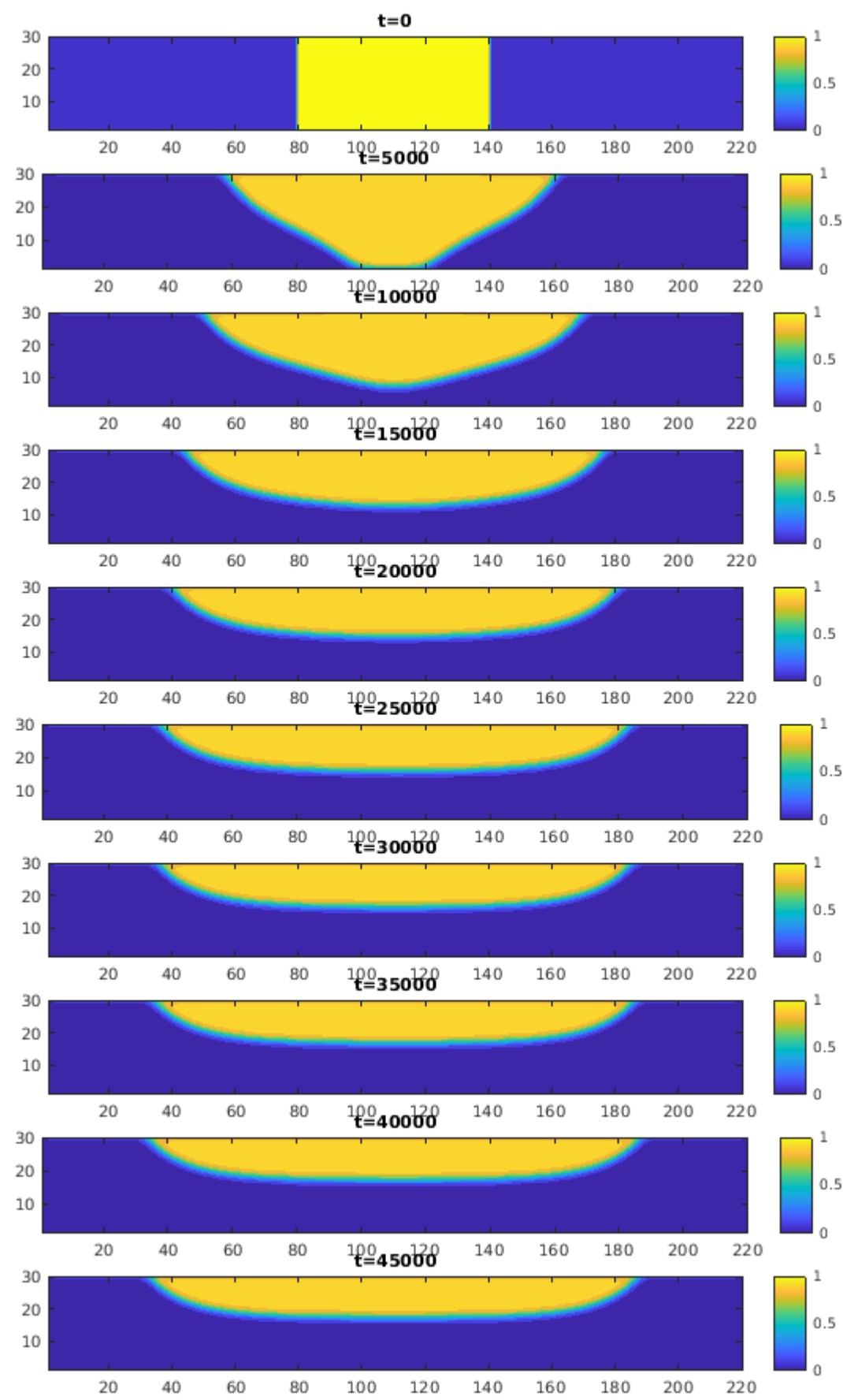

Figure 5.7: Initialization of a droplet of density $\rho_{1}=1.0$ and viscosity $\nu_{1}=0.33$ surrounded by a fluid of density $\rho_{2}=0.77$ and viscosity $\nu_{2}=0.33$ when $\Delta \theta=130$. Dimensions in $[\mu \mathrm{m}]$ and each time step is $31.622[\mathrm{~ns}]$. 


\subsection{Uneven Wettability Dynamic Effect}

After the droplet initialization step (Section 5.1) is complete, the dynamic impact of the uneven wetting conditions is observed by triggering the flow. All geometric parameters and hydrodynamic properties in this section are similar to those in the initialization process in Section 5.1. In addition, the aspect ratio, $A R=D / W$, and the side to main channel width ratio, $W R=W_{1} / W$ are fixed at unity. The capillary and Reynolds numbers are kept constant at $\mathrm{Ca}=0.029$ and $\mathrm{Re}=0.455$ respectively.

Figure 5.8 shows how the droplet develops when $\theta_{\text {lower }}=173.2 \pm 7.1$ and $\theta_{\text {upper }}=$ $143.2 \pm 0.8$. As the continuous phase is discharged from the inlet, the droplet is displaced towards the bifurcation of the T-junction. When it enters the bifurcation, after $\mathrm{t}=200000$ steps, it spreads in both directions of the side channel. Eventually, it leaves the main channel completely and blocks the inlet flow. At the same time, the pressure upstream in the main channel grows due to the accumulation of the continuous phase fed from the inlet. The increase in pressure deforms the droplet gradually starting at the mid-plane of the channel. This thinning in the droplet neck leads to an increase in the curvature at the middle of the channel and the thinnest region is not at the middle of the droplet due to the the asymmetric positioning from uneven wetting. As the neck curvatures exceeds channel dimension the droplet splits into two unequal droplets with the larger drop on the side of higher wettability. The evolution of the droplet neck starting from squeezing to rupturing is consistent with the works of even wettability conditions in literature $[82,86,87]$. Figure 5.8 shows that even with about a $30^{\circ}$ difference in contact angles, drop breakup is possible. While breakup is interesting, it is inherently the opposite of separation.

Figure 5.9 for the case of $\theta_{\text {upper }}=120.4 \pm 0.7$ and $\theta_{\text {lower }}=173.2 \pm 7.1$ shows this the droplet is displaced to the upper half due to the increase of wettability difference 
which shifts the neck towards the lower end of the droplets away from its center. However, the droplet is still able to prevent the continuous phase from flowing to the side channels for a while. During that time, and due to the pressure increase in the main section, the lower end of the channel is detached from the side wall near the main channel leading to a fast escape of the fluid trapped upstream to the lower side channel. The sudden flow through the emerging tunnel enhances the deformation rate due to viscous shear stress. Consequently, the droplet end is stretched to the limit where the viscous shear forces overcome the surface tension resistance. Eventually, droplet breakup occurs as if it was an unconfined drop. The major part of the drop is driven to the upper half while the remaining daughter drop is carried to the lower half.

Increasing the wettability of the upper half by setting $\theta_{\text {upper }}=103.2 \pm 0.6$ should inhibit drop breakup with the droplet transported to the upper side completely. As seen from Figure 5.10, the droplet does not hinder the flow as the lower end is not positioned enough to block the corresponding side channel; therefore, a tunnel opens very early and the droplet is squeezed to the other side. A similar result is obtained from $\theta_{\text {upper }}=95.2 \pm 0.4$ (see Fig.5.11) but the with lower tunnel opening faster due to the greater wettability difference.

Figures 5.12 to 5.14 show three cases where $\theta_{\text {upper }}=83.2 \pm 0.3,69.1 \pm 0.4$, and $43.0 \pm 0.2$. In each the droplets move down stream without any contact with the lower wall of the main channel. As a result, the lower side channel is not obstructed at any moment during the droplet transition from the main channel to the side channels. For this reason the droplets always move to the upper side without any impedance at the T-junction. Moreover, the tail of the droplet is delayed by the effect of adhesion force between the droplet and the upper half. At $\theta_{\text {upper }}=43.0 \pm 0.2$ (Figure 5.14) it is visible that the adhesion effect overcomes the cohesion forces that the tail is left 
behind the mother droplet under the viscous shearing effect.

To summarize, the wettability difference required to drive the droplet to one side of the T-junction is achieved by $\Delta \theta>70^{\circ}$. For these cases the droplet length, $L_{0}=2 \mathrm{~W}$, and the capillary number, $\mathrm{Ca}=0.029$. The effects of droplet length and capillary number on droplet dynamics under uneven wetting conditions need to be determined. 

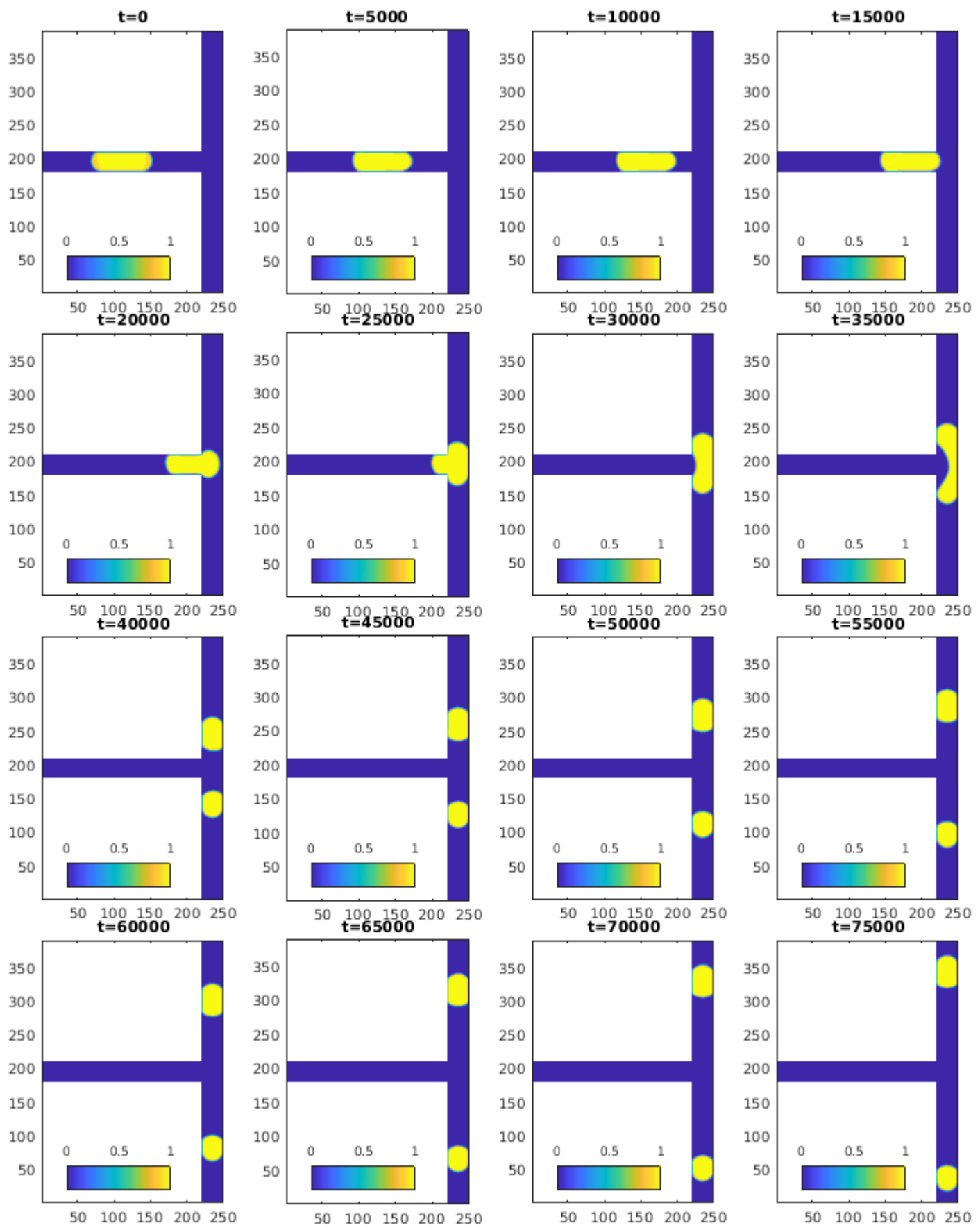

Figure 5.8: Hydrodynamic response of a droplet of density $\rho_{1}=1.0$ and viscosity $\nu_{1}=0.33$ surrounded by a fluid of density $\rho_{2}=0.77$ and viscosity $\nu_{2}=0.33$ when $\Delta \theta=30$ at capillary number $C a=0.029$ corresponding to Reynolds number $R e=$ 0.455. Dimensions in $[\mu m]$ and each time step is $31.622[\mathrm{~ns}]$. 

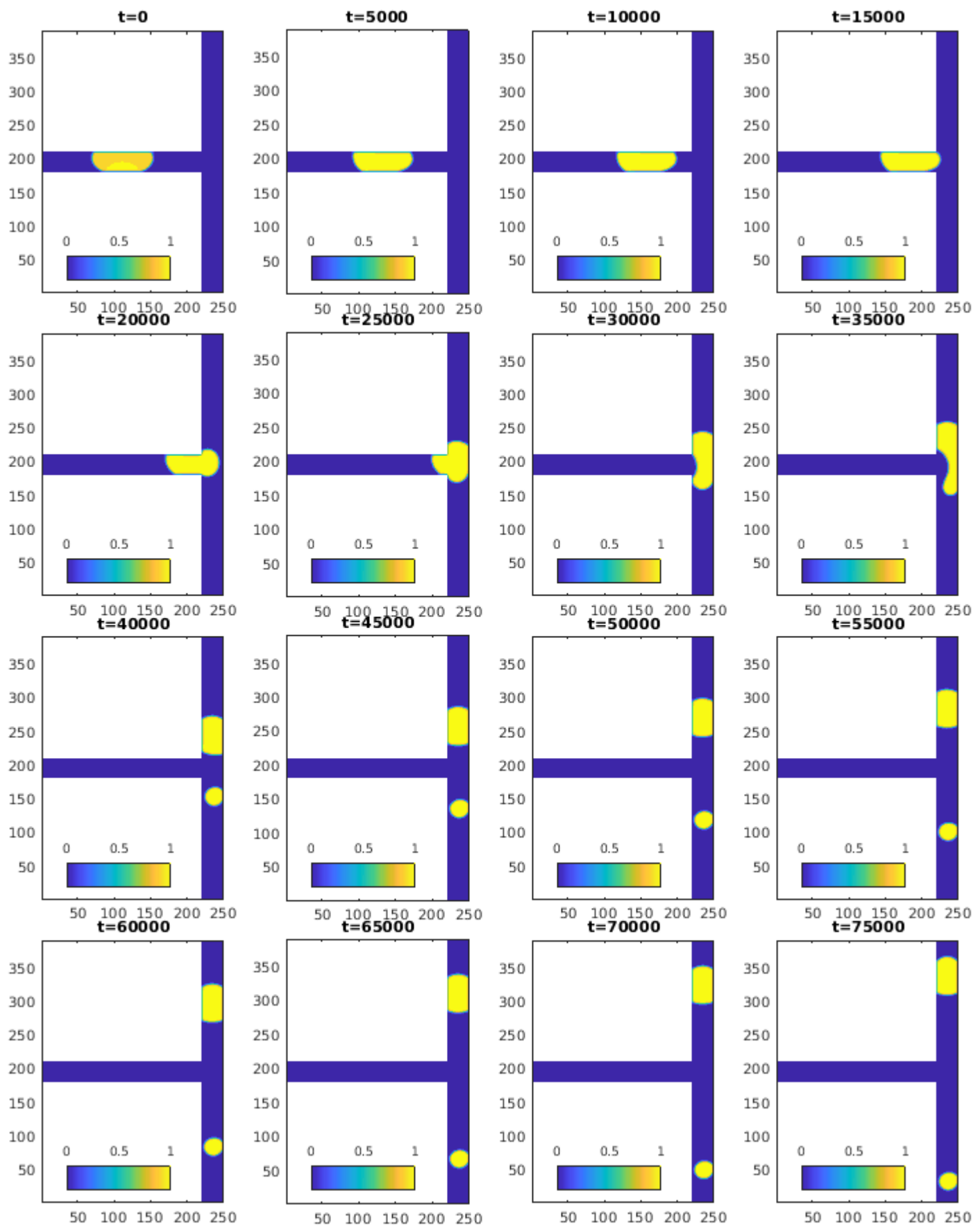

Figure 5.9: Hydrodynamic response of a droplet of density $\rho_{1}=1.0$ and viscosity $\nu_{1}=0.33$ surrounded by a fluid of density $\rho_{2}=0.77$ and viscosity $\nu_{2}=0.33$ when $\Delta \theta=53$ at capillary number $C a=0.029$ corresponding to Reynolds number $R e=$ 0.455. Dimensions in $[\mu m]$ and each time step is $31.622[\mathrm{~ns}]$. 

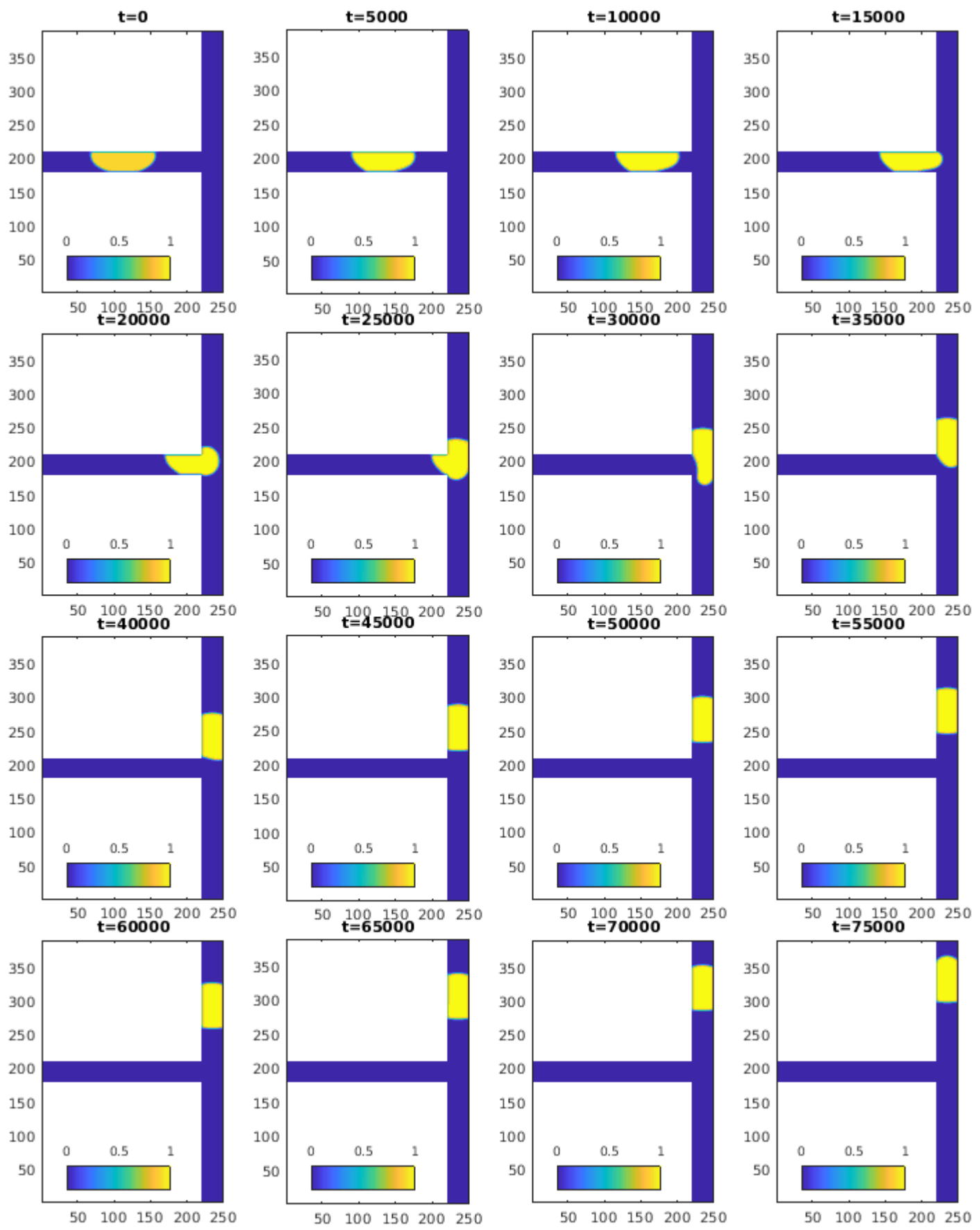

Figure 5.10: Hydrodynamic response of a droplet of density $\rho_{1}=1.0$ and viscosity $\nu_{1}=0.33$ surrounded by a fluid of density $\rho_{2}=0.77$ and viscosity $\nu_{2}=0.33$ when $\Delta \theta=70$ at capillary number $C a=0.029$ corresponding to Reynolds number $R e=$ 0.455. Dimensions in $[\mu m]$ and each time step is $31.622[\mathrm{~ns}]$. 

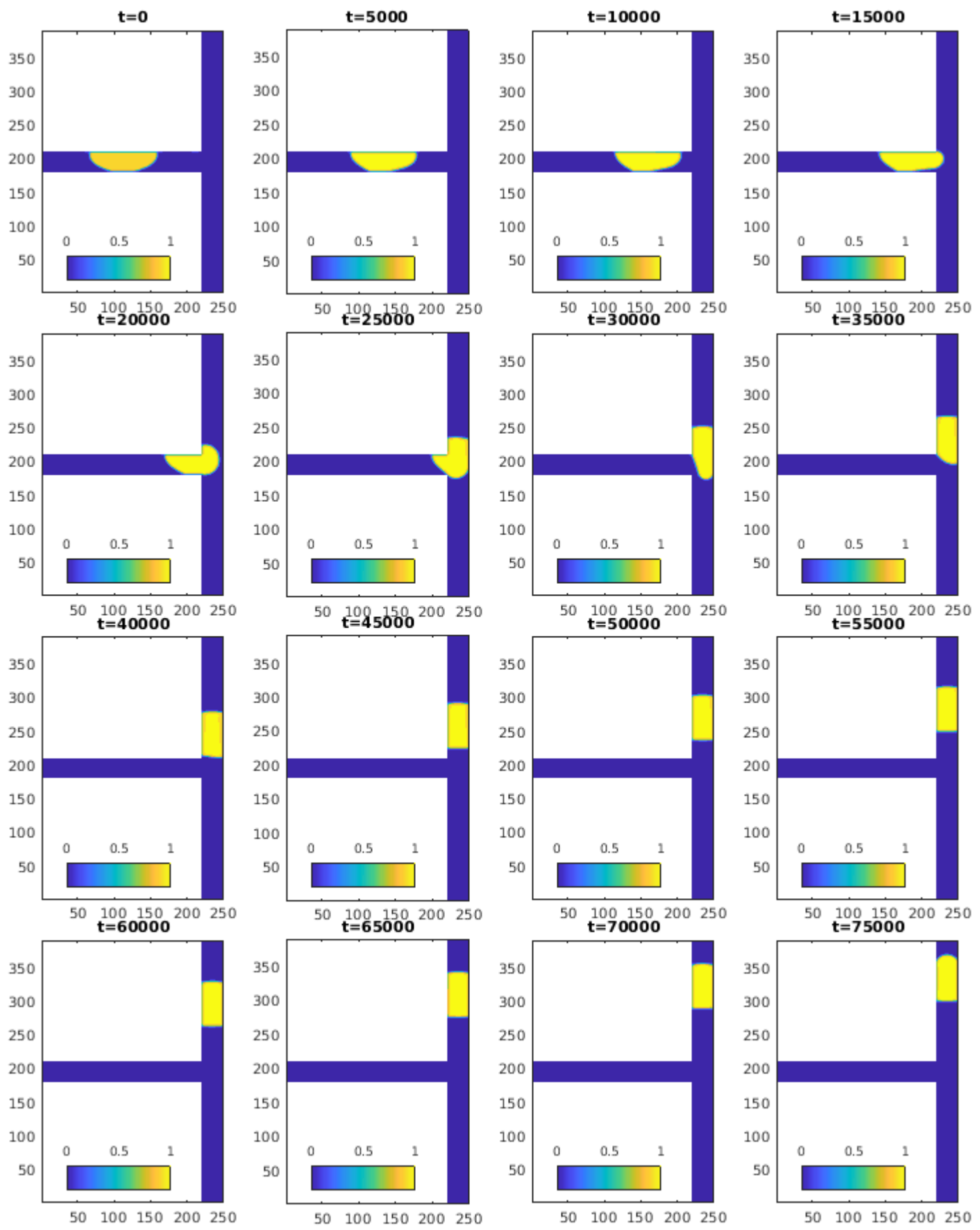

Figure 5.11: Hydrodynamic response of a droplet of density $\rho_{1}=1.0$ and viscosity $\nu_{1}=0.33$ surrounded by a fluid of density $\rho_{2}=0.77$ and viscosity $\nu_{2}=0.33$ when $\Delta \theta=78$ at capillary number $C a=0.029$ corresponding to Reynolds number $R e=$ 0.455. Dimensions in $[\mu m]$ and each time step is $31.622[\mathrm{~ns}]$. 

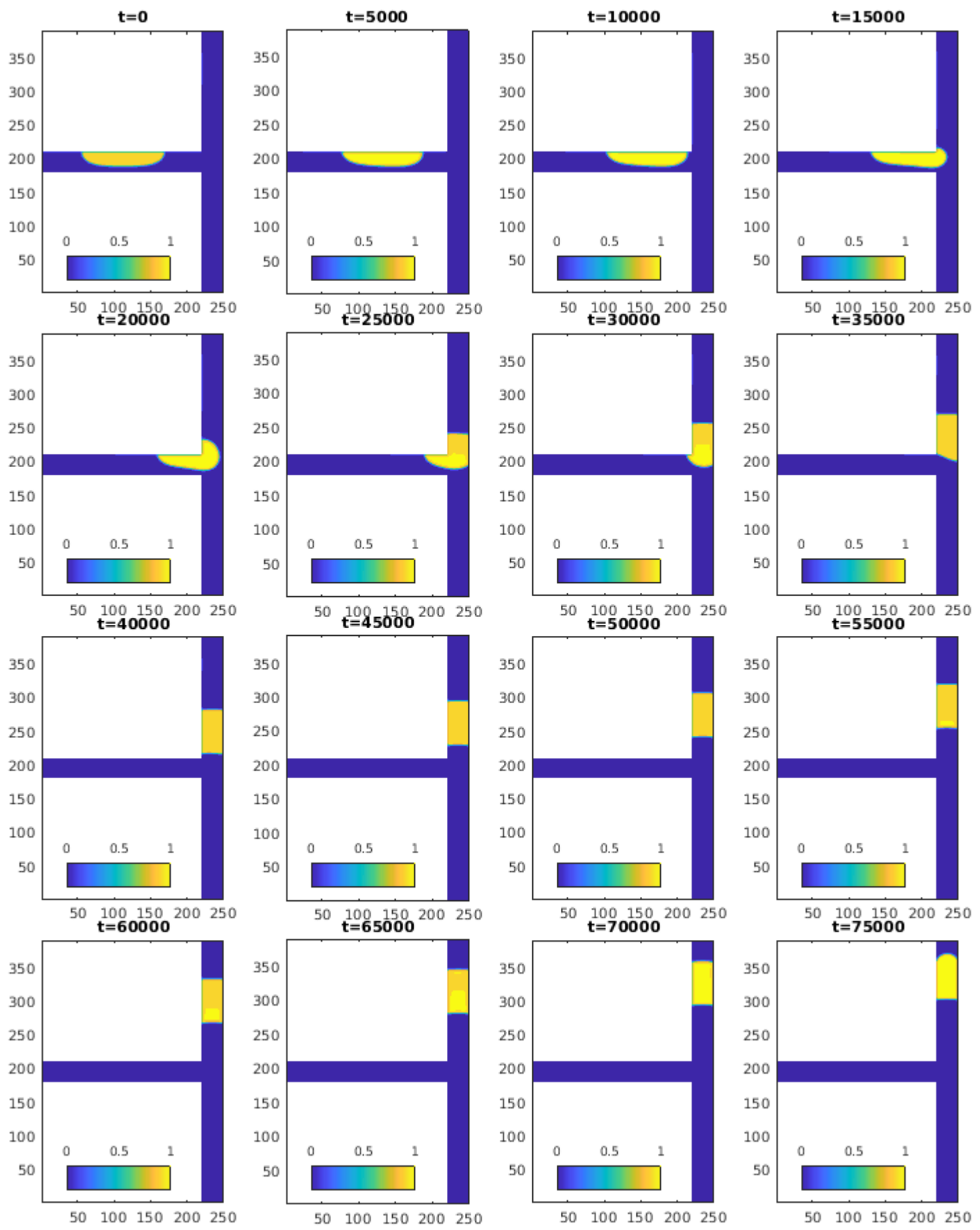

Figure 5.12: Hydrodynamic response of a droplet of density $\rho_{1}=1.0$ and viscosity $\nu_{1}=0.33$ surrounded by a fluid of density $\rho_{2}=0.77$ and viscosity $\nu_{2}=0.33$ when $\Delta \theta=90$ at capillary number $C a=0.029$ corresponding to Reynolds number $R e=$ 0.455. Dimensions in $[\mu m]$ and each time step is $31.622[\mathrm{~ns}]$. 

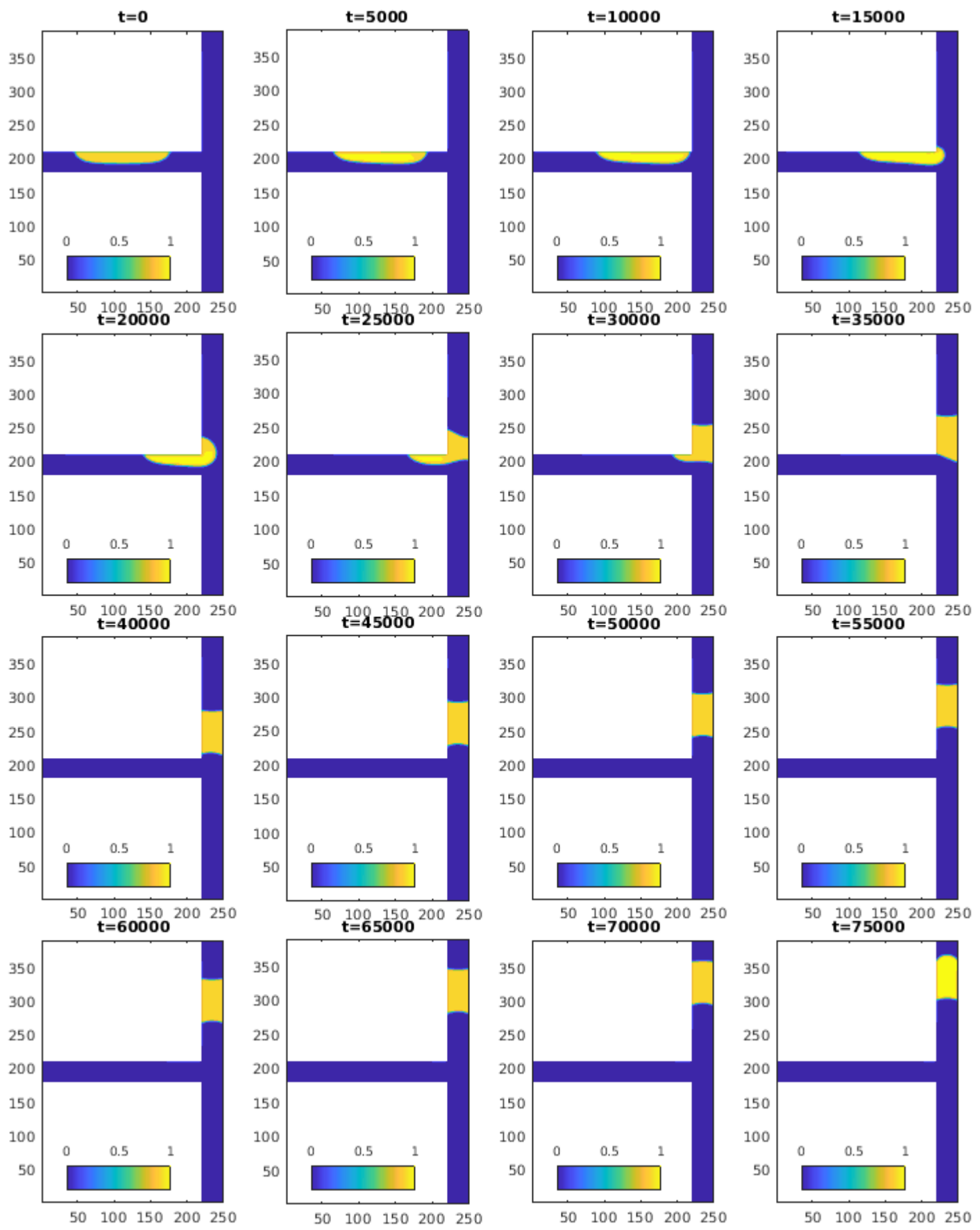

Figure 5.13: Hydrodynamic response of a droplet of density $\rho_{1}=1.0$ and viscosity $\nu_{1}=0.33$ surrounded by a fluid of density $\rho_{2}=0.77$ and viscosity $\nu_{2}=0.33$ when $\Delta \theta=104$ at capillary number $C a=0.029$ corresponding to Reynolds number $R e=$ 0.455. Dimensions in $[\mu m]$ and each time step is $31.622[\mathrm{~ns}]$. 

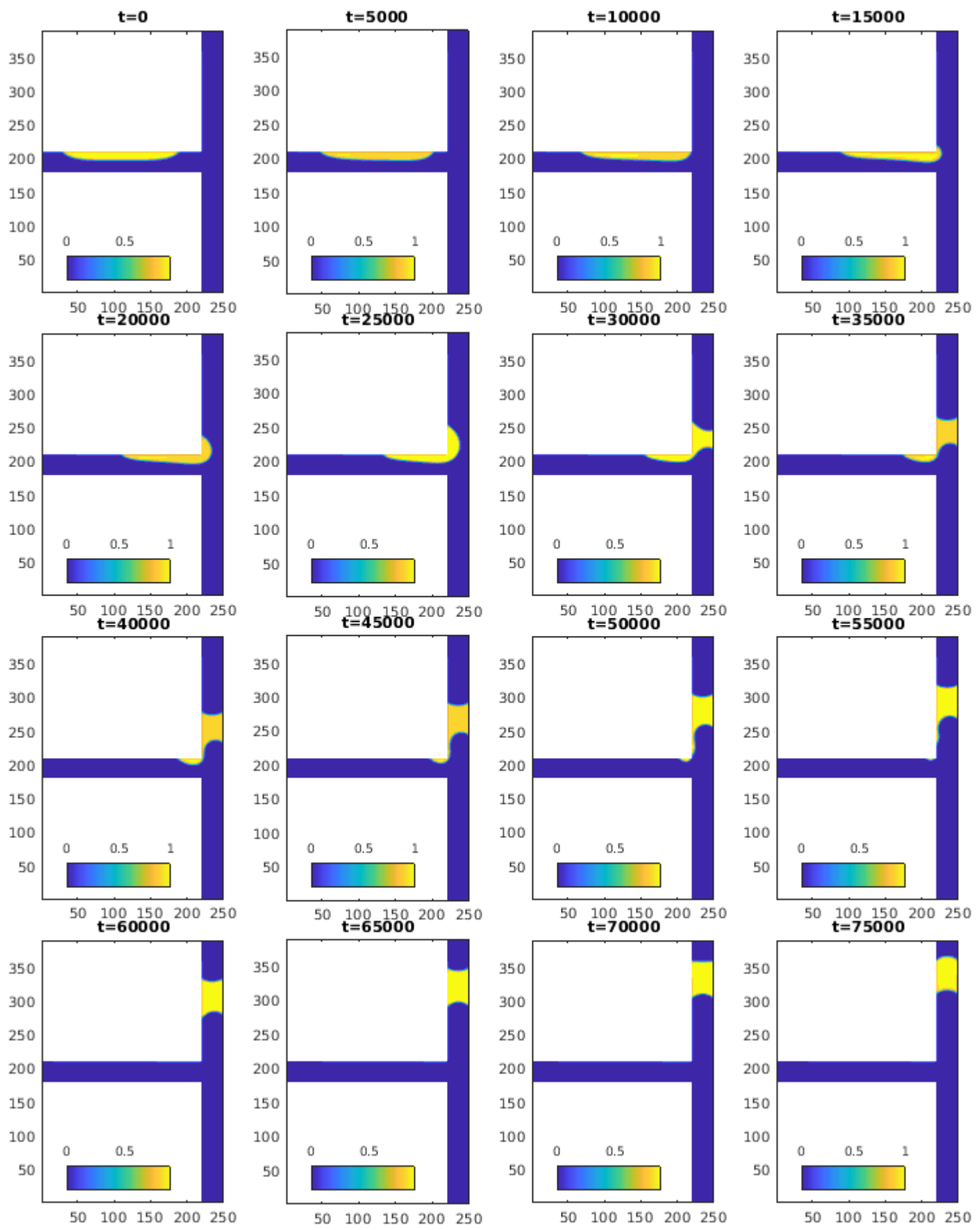

Figure 5.14: Hydrodynamic response of a droplet of density $\rho_{1}=1.0$ and viscosity $\nu_{1}=0.33$ surrounded by a fluid of density $\rho_{2}=0.77$ and viscosity $\nu_{2}=0.33$ when $\Delta \theta=130$ at capillary number $C a=0.029$ corresponding to Reynolds number $R e=$ 0.455. Dimensions in $[\mu m]$ and each time step is $31.622[\mathrm{~ns}]$. 


\subsection{Initial Length and Capillary Number Effect}

In the preceding section we examined the dynamic evolution of the droplet under variable wetting conditions, but at fixed initial droplet length, $L_{0}$, and capillary number, Ca. In this section we deal with the droplets dynamics under fixed wetting conditions with variable $L_{0}$ and $\mathrm{Ca}$. The case tested here is chosen to be in the middle of the tested range of the upper half contact angle, $\theta_{\text {upper }}=95.2 \pm 0.4$, while the lower section contact angle is fixed at $\theta_{\text {lower }}=173.2 \pm 7.1$.

The effect of the dimensionless droplet length, $L_{0} / W$, is shown in Figure 5.15 when $\mathrm{Ca}=0.029$. The values of $L_{0} / W$ covered here are 2, 3, and 4 corresponding to the first, second, and third rows in the figure. It is apparent that increasing the droplet length not only triggers the the breakup but also increases the size of the daughter droplet which is driven to the lower side channel. Figure 5.15 shows all droplet enter the bifurcation in the same way where the front end swerves to the upper side channel due to the wettability difference. However, for longer droplets, there is a period when the upper side channel is completely blocked with the trailing end still in the main channel. At this point, the tail of the droplet is compelled to enter the other side of higher contact angle (lower wettability). Whether the lower side channel is partially or fully obstructed by the droplet, drop breakup occurs.

Figure 5.16 shows the effect of capillary number on the breakup at fixed droplet length, $L_{0}=4 W$. The three cases considered here are $\mathrm{Ca}=0.029,0.058$, and 0.116 corresponding to the first, second, and third rows respectively. The value of $\mathrm{Ca}$ is solely changed by modifying the flow velocity and fixing the viscosities and surface tension. The droplet breaks in all cases. As the Ca increases, the size difference between the daughter and the mother droplets decreases. This is because the pressure growth in the main channel develops faster than the interfacial effect arising from 
wetting asymmetry in the side channels.
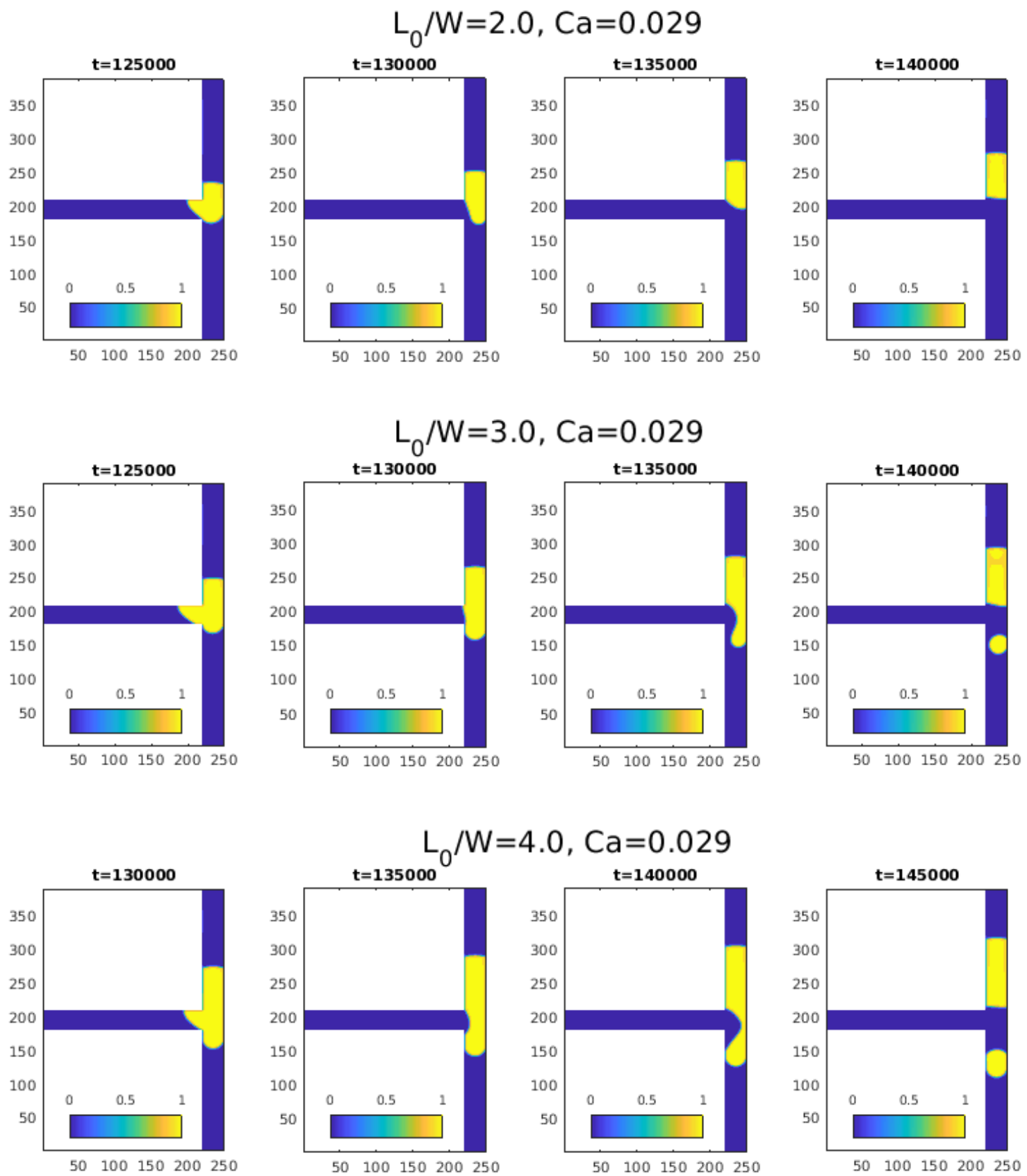

Figure 5.15: Effect of the initial droplet length on the droplet break up when $\theta_{\text {upper }}=$ $95.2 \pm 0.4$ and $\theta_{\text {lower }}=173.2 \pm 7.1$ at capillary number $\mathrm{Ca}=0.029$. Dimensions in $[\mu \mathrm{m}]$ and each time step is $31.622[\mathrm{~ns}]$. 

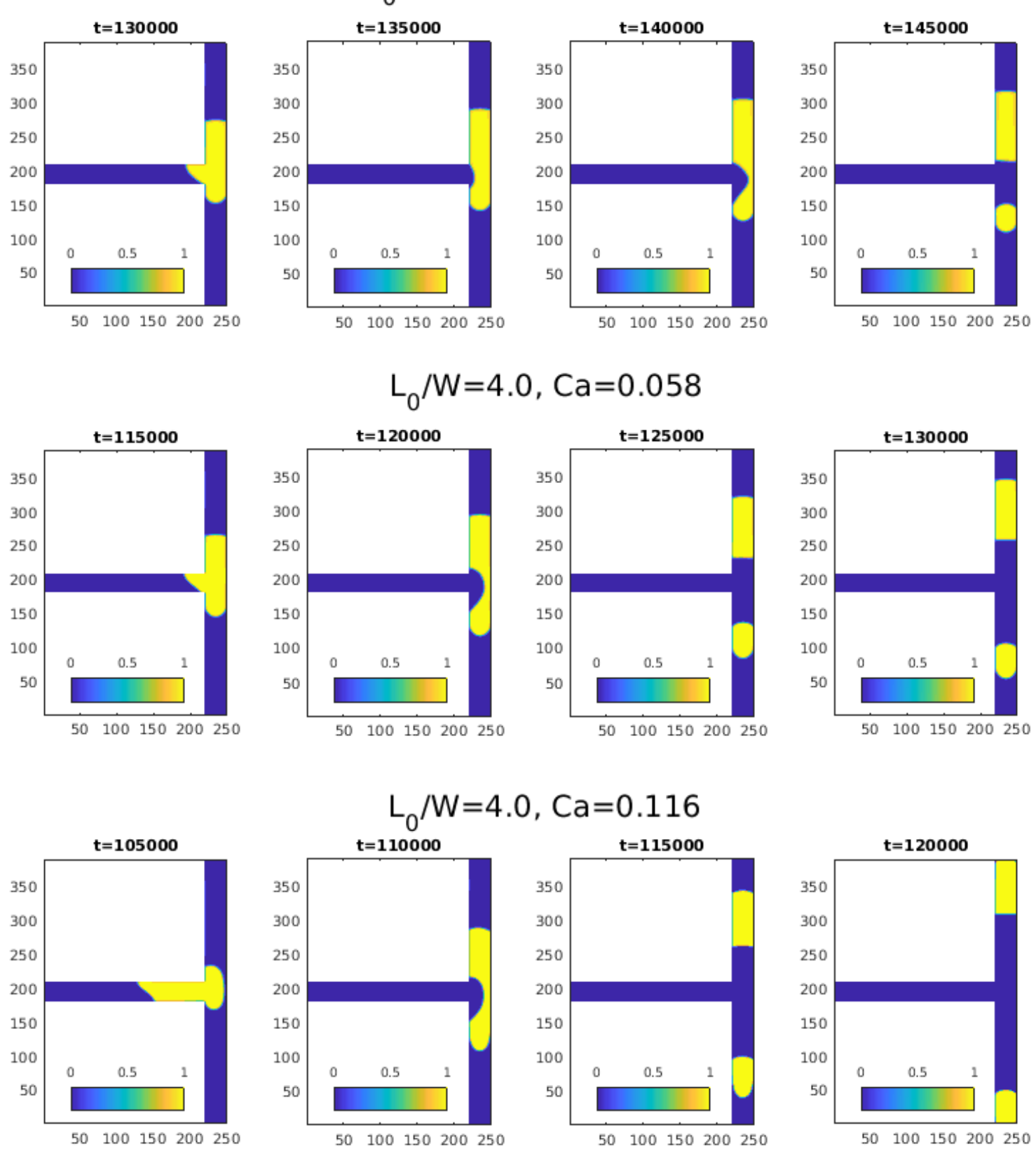

Figure 5.16: Effect of capillary number on the droplet break up when $\theta_{\text {upper }}=95.2 \pm$ 0.4 and $\theta_{\text {lower }}=173.2 \pm 7.1$ at a droplet length $L_{0}=4 W$. Dimensions in $[\mu m]$ and each time step is $31.622[\mathrm{~ns}]$. 


\subsection{Regimes of Results}

From the previous sections, it is apparent that the threshold of drop breakup is determined by the wetting conditions, $\mathrm{Ca}$, and $L_{0}$. Identifying the thresholds induced by a range of the controlling parameter is useful for creating the regimes of breakup and nonbreakup. Consequently, the feasibility of separation depends on the given parameters. However, the regimes are sensitive to the hydrodynamic properties of the participating fluids and the geometric parameter of the confining channel. Therefore we establish the Ca- $L_{0}$ drop behavior diagrams under the effect of wettability difference, droplet to outer fluid viscosity ratio $\lambda$, channel aspect ratio $\mathrm{AR}$, and side to main channel width ratio WR.

\subsection{Effect of Wetting Conditions}

The effect of changing the upper section wetting conditions on drop breakup is considered here for upper-section contact angle values of are $\theta_{\text {upper }}=173.2 \pm 7.1,95.2 \pm 0.4$, and $43.0 \pm 0.2$ while $\theta_{\text {lower }}=173.2 \pm 7.1$. The parameters such as $\lambda, A R$, and WR are set to 1.0 for all three cases.

Figure 5.17 provides the breakup and nonbreakup regimes. Red triangles represent breakup and blue circles represent non breakup. When the entire channel has identical wettability, corresponding to the case $\theta_{\text {upper }}=\theta_{\text {lower }}=173.2 \pm 7.1$ shown in Figure 5.17 (a), the breakup regime dominates the phase diagram and only in a very limited number of simulation does the droplet not break. The no breakup region is restricted to low Ca and short drop length where the T-junction is not fully obstructed by the droplet. One may notice that the border between the two regimes is shifted to left due to the use of initial droplet length instead of the final length because the former is consistent in all the uneven wettability conditions while the latter is not. 
When the magnitude $\Delta \theta=\theta_{\text {lower }}-\theta_{\text {upper }}$ increases the breakup regime reduces significantly as the droplet is shunted to upper side channel by the interfacial asymmetry as seen from Figure 5.17 (b) and Figure 5.17 (c). With $\theta_{\text {upper }}=43.0 \pm 0.2$ no breakup would be expected for the entire ranges of $\mathrm{Ca}$ and $L_{0}$ which makes this case as a perfect candidate for separation purposes.

Figures 5.18 and 5.19 show the Ca- $L_{0}$ droplet behavior diagrams from experimental studies of segmented flows in T-junctions with even wetting conditions, reported in the literature. Figure 5.18 is adapted from Jullien et al [88] for viscosity ratio (a) $\lambda=0.11$ and (b) $\lambda=1.67$. Figure 5.19 is adapted from Link et al [89] and it is related to water drops dispersed in Hexadecane (the resulting viscosity contrast $\lambda=0.11$ ). Figure 5.17 agrees well with Figures 5.18(b). The shift to the left is because we are using the initial length of the droplet. 


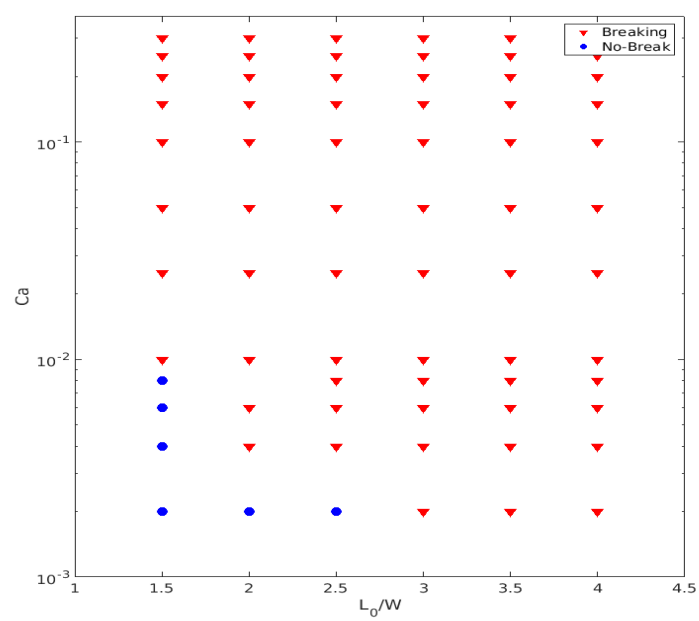

(a) $\Delta \theta=0$

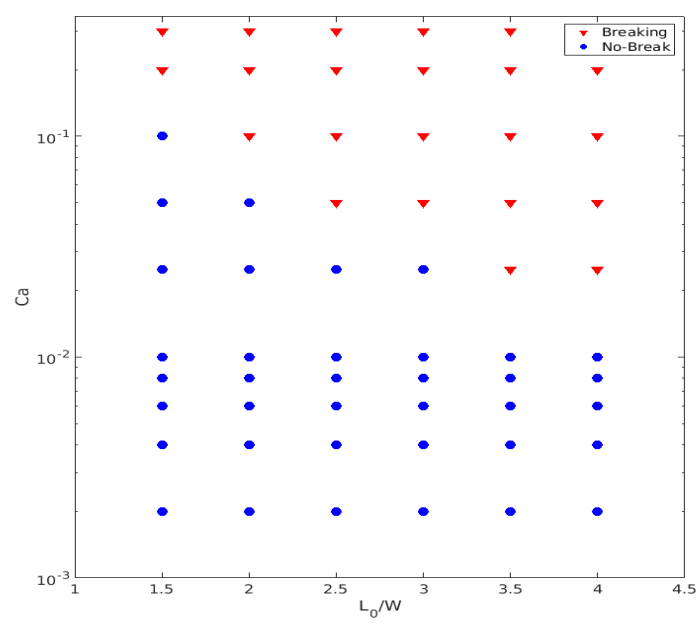

(b) $\Delta \theta=78$

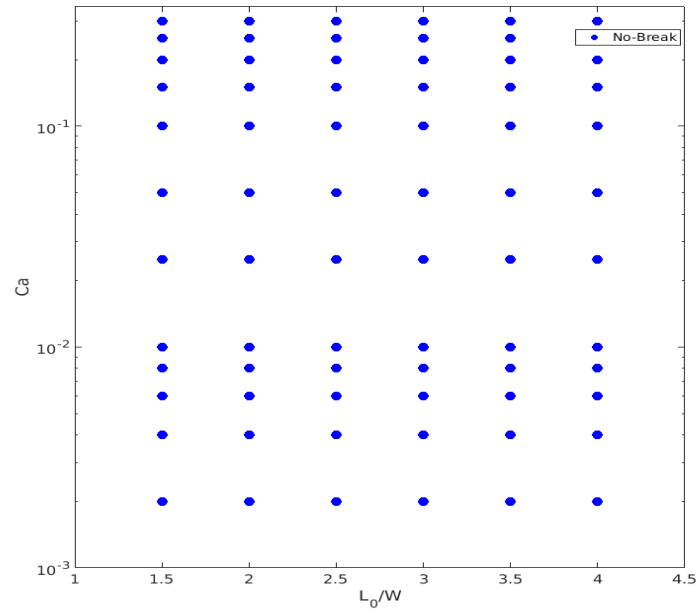

(c) $\Delta \theta=130$

Figure 5.17: Break up and no-break up regimes under different capillary numbers and drop length for three cases of $\Delta \theta$. 

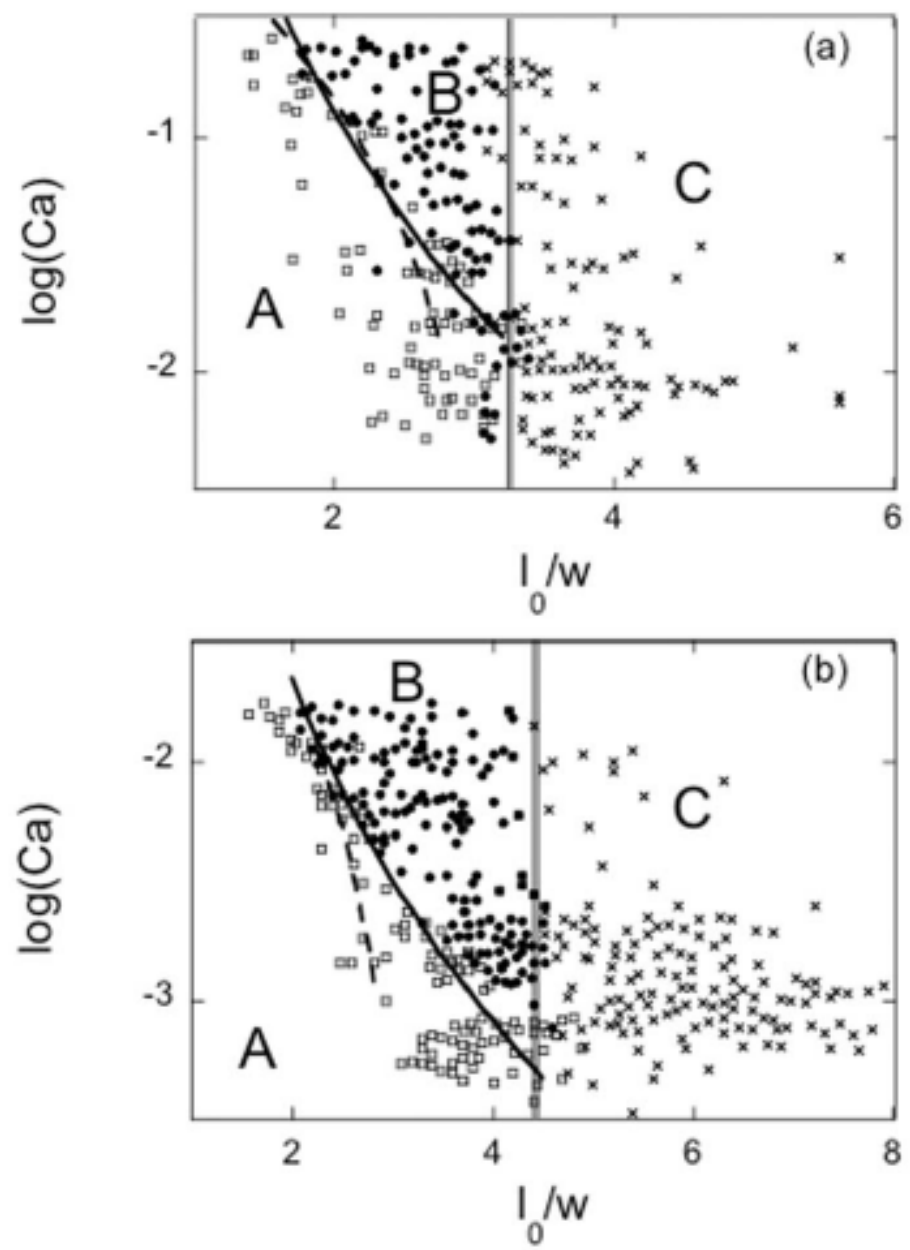

Figure 5.18: The Ca- $L_{0}$ diagrams for drop flows in T-junction from Jullein et al's experimental study [88] for (a) $\lambda=0.11$; (b) $\lambda=1.67$. A, B, and $\mathrm{C}$ are no-breakup, breakup with tunnels, and breakup with permanent obstruction regimes. 


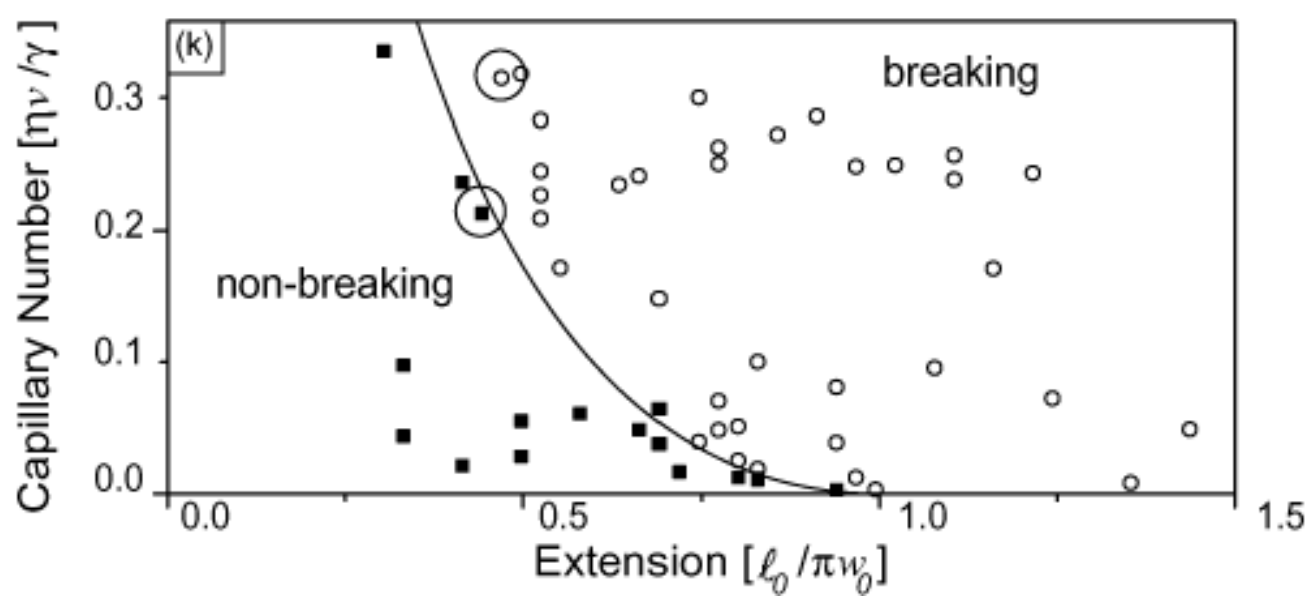

Figure 5.19: The Ca- $L_{0}$ diagrams for water (viscosity $\mu=0.894 \mathrm{mPas}$ ) droplets dispersed in Hexadecane+SPAN80 (viscosity $\mu=8.0 \mathrm{mPas}$ ) continuous flow in Tjunction from [89]

\subsection{Effect of Viscosity Ratio}

The viscous effect is the main competition to the surface tension force and hence is a major player in the breakup process. The ratio of the droplet to outer fluid viscosity, $\lambda$, is employed here to characterize the importance of viscous forces in the process. Three different case are considered by changing the droplet viscosity and fixing the continuous phase viscosity to produce $\lambda=0.12,1.2$, and 2.0. The wetting conditions are fixed such that $\theta_{\text {upper }}=95.2 \pm 0.4$ and $\theta_{\text {lower }}=173.2 \pm 7.1$ for all cases. In addition, the aspect and width ratios are taken as unity.

Figure 5.20 provides the Ca- $L_{0}$ drop behavior diagrams for the viscosity ratios of $\lambda=0.12,1.2$, and 2.0. The impact of $\lambda$ on the breakup regime is double-sided which means more breakups are experienced by long droplet while the short ones are more likely to survive. In order to understand the reason of this result, we need to refer back to the literature of droplets breakup under even wetting conditions. Hoang et al [82] noticed that vortical flows inside droplet contribute to nonbreakup due to the deformation resistance compared to droplets without rotational flow inside. When 
the inner viscosity increases that would suppress the vorticity due to the increased viscous dissipation which interprets why longer droplet are broken when $\lambda$ increases. In addition, the same study revealed that raising the droplet viscosity would reduce the pinching rate which in our situation is exploited by the wettability asymmetry leading to droplet escape to higher wettability side. Therefore, the separation is more feasible on the left side of the diagram while it is less recommended on the right side.

From what is mentioned above, it is evident that the strengthened tendency to breakup due to the increased $\lambda$ is extenuated by the difference in wetting conditions which, the latter, acts as a driving force for non-breakup. 


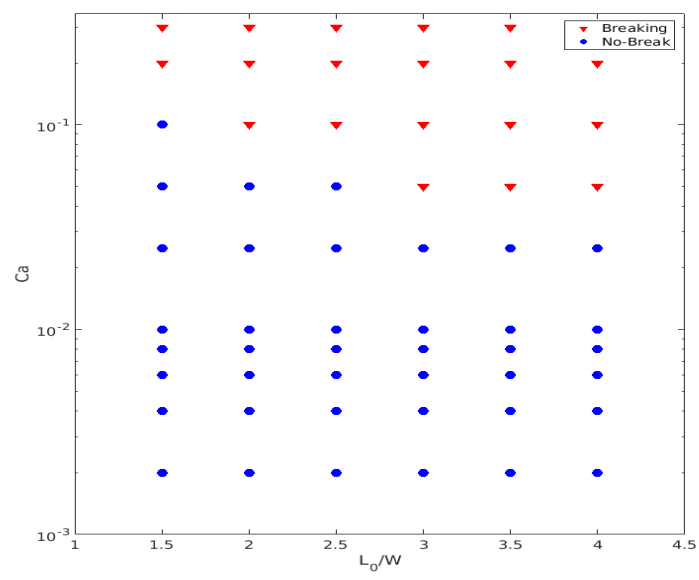

(a) $\lambda=0.12$

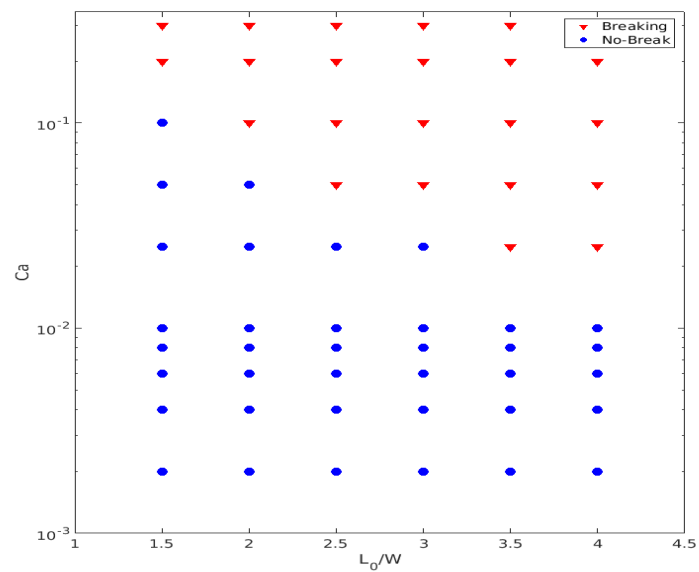

(b) $\lambda=1.2$

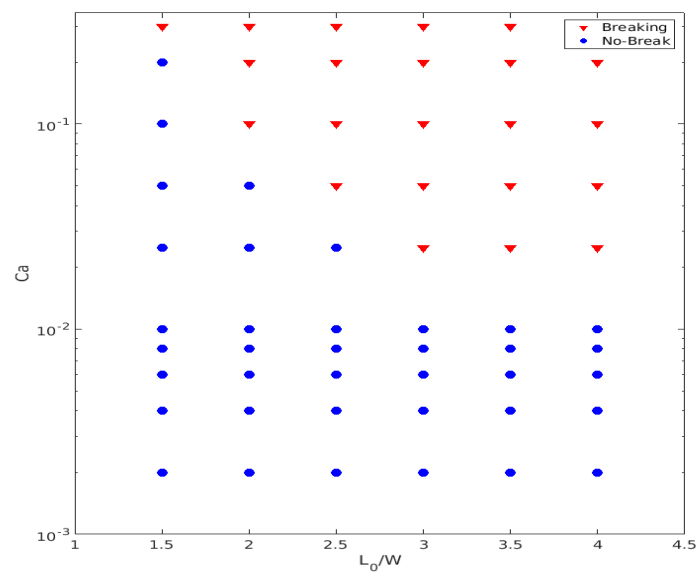

(c) $\lambda=2.0$

Figure 5.20: Effect of the viscosity ratio $\lambda$ on break up and no-break up regimes for different capillary numbers and drop length. 


\subsection{Effect of Aspect Ratio}

In addition to the interfacial tension, the geometry of confined drops are also governed by the the shape of the confining surfaces. Accordingly, the surface area and topology of the droplet are determined by the dimensions of the channel. One of the most important parameters is the main channel aspect ration, AR. The value of $A R$ is varied by altering the depth of the channel, D, and fixing the width, W. The wetting conditions are fixed such that $\theta_{\text {upper }}=95.2 \pm 0.4$ and $\theta_{\text {lower }}=173.2 \pm 7.1$ when $\lambda=1.0$.

Figure 5.21 provides drop diagrams for three aspect ratios. At $\mathrm{AR}=0.5$ shown in Figure 5.21(a), the droplets pass to the upper side channel without any breakup in most of the cases. Only long droplets, nearly $L_{0}=4 W$, under high flow velocity, or high Ca alternatively, experience breakup. When $\mathrm{AR}$ is raised to 1.0, shown in Figure 5.21(b), the breakup area expands significantly. However, when $\mathrm{AR}=1.5$ as visible from Figure 5.21(c) the growth in the breakup area is nearly marginal. This is consistent with the findings in the literature of breakup under even wetting conditions $[82,90]$. The reason is that the critical neck thickness, $\delta_{c r}$ is found to depend on the aspect ratio as $\delta_{c r}=\frac{A R}{A R+1}$. Thereby, the droplet in a channel of $\mathrm{AR}=0.5$ has to reach $\delta_{c r}=0.33 \mathrm{~W}$ in order for the breakup to happen. When $\mathrm{AR}$ is greater than 1.0 the breakup occurs earlier because $\delta_{c r}$ approaches $\mathrm{W}$.

An interpretation of the effect of $\mathrm{AR}$ is the contribution of tunnels in extending the droplet during the neck thinning. As AR decrease the depth of the channel, D, decreases and the flow of the continuous phase through the tunnels near the main channel is restricted. The decreased flow through the thin layers lowers the the deformation rate imposed by viscous shear. The time required for the droplet to breakup when $\mathrm{AR}=0.5$ is longer than that when $\mathrm{AR}=1.5$. During that time the 
effects of wetting asymmetry counteract the ability of tunnel flows. 


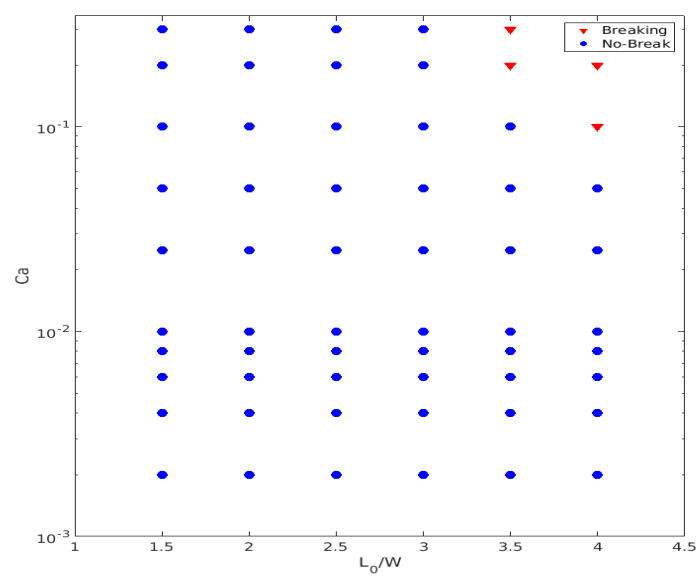

(a) $A R=0.5$

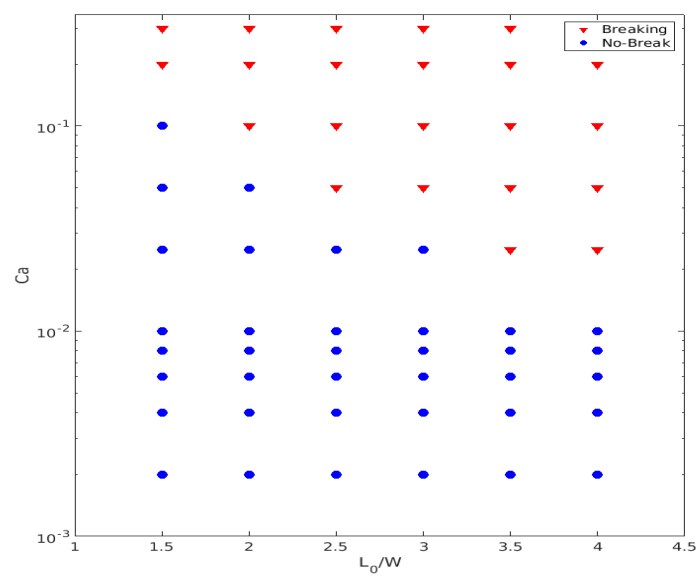

(b) $A R=1.0$

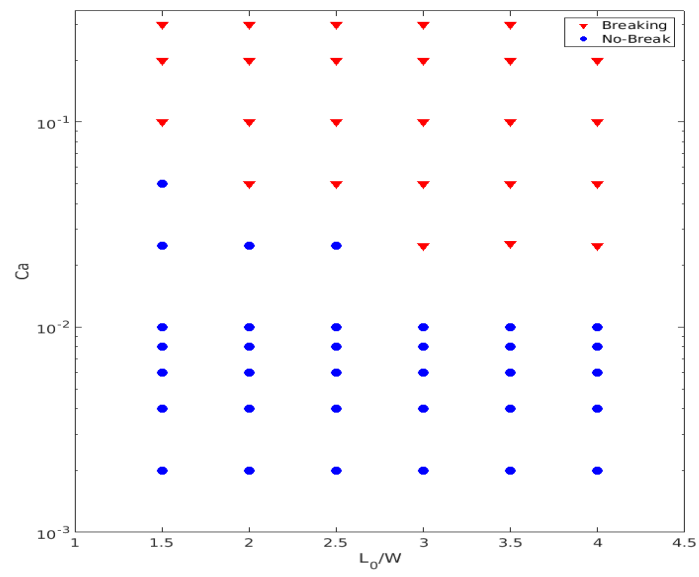

(c) $A R=1.5$

Figure 5.21: Effect of the channel aspect ratio AR on break up and no-break up regimes for different capillary numbers and drop length. 


\subsection{Effect of Side to Main Channel Width Ratio}

In this section we discuss the effect of side to main channel width ratio, WR. This parameter is controlled by fixing the main channel width, $\mathrm{W}$, and modifying the side channel width, W1. The tested values of WR $0.5,1.0$, and 1.5 while all other parameters such as $\lambda$ and AR are set at 1 . The wetting conditions are fixed such that $\theta_{\text {upper }}=95.2 \pm 0.4$ and $\theta_{\text {lower }}=173.2 \pm 7.1$.

The Ca- $L_{0}$ results due to variation of WR are displayed in Figure 5.22. It is discernible that increasing the slenderness of the side channel magnifies the breakup area. On the other hand, as WR increases the non-breakup regime dominates the diagram. Opposite the effect of AR effect in the previous section, as we increase WR by extending W1 the interaction area between the solid wall and the droplet is increased and surface force arising from the wettability difference is increased. In addition, when the droplet leaves the main channel at $\mathrm{WR}>1$, it finds its self faraway from the back wall which delays obstruction of the upper side channel until the last stage of the transition, a moment when the continuous phase already starts to flow to the lower side channel.

When combined together, the high WR and low AR are expected to achieve superior results in separations of two-phase flows even for small wettability difference between the upper and lower halves of a channel. 


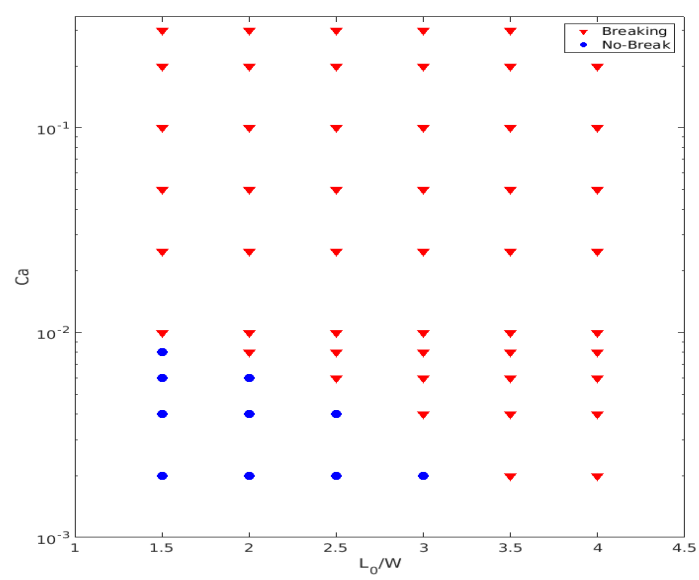

(a) $W R=0.5$

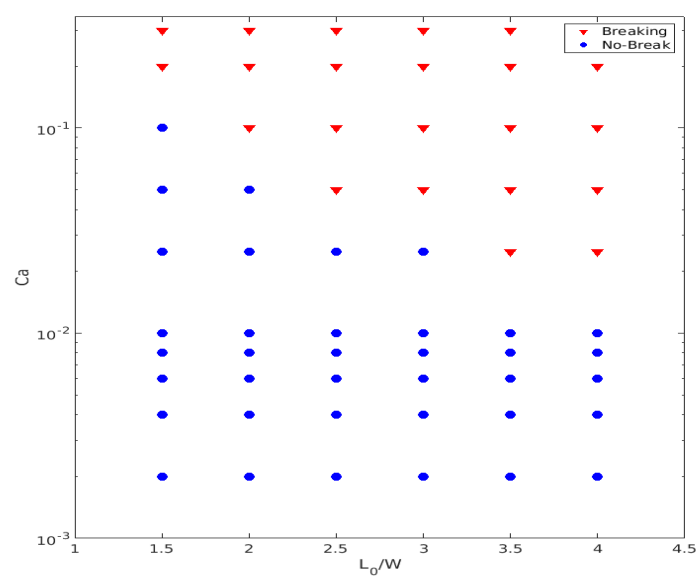

(b) $W R=1.0$

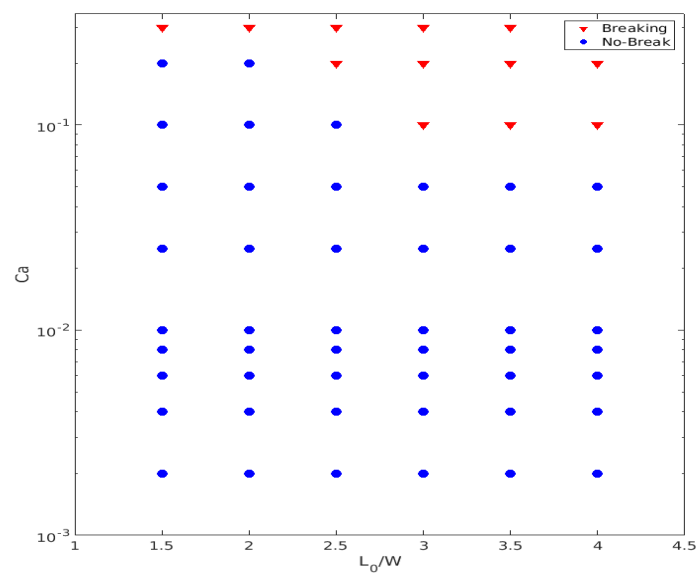

(c) $W R=1.5$

Figure 5.22: Effect of the side to main channel width ratio AR on break up and no-break up regimes for different capillary numbers and drop length. 


\section{Chapter 6}

\section{Conclusions and Recommendations}

In this work, we applied Lattice Boltzmann Method (LBM) based on the Shan-Chen model to explore the effects of interfacial forces on pre-concentration and separation of binary fluid mixtures in microchannels. For the miscible case, in which a mixture of ethanol and water was simulated, the D2Q9 lattice set was employed with multiple lattice belts to describe the water-ethanol, water-solid, and ethanol-solid interactions. Before diving into interactions and the impacts, we validated the model by comparing the computed mixture velocity profile with the analytic solution of Navier-Stoke equation for 2D pressure driven flow between parallel plates. The model agreed well with the analytic solution with less than $17 \%$ maximum relative error near the boundaries. For the immiscible case, the D3Q27 lattice set with Shan-Chen LBM model was used to study two-phase flow separation in a 3D T-shaped microchannel where the contact angles of the upper and lower boundaries are adjusted by varying the interaction coefficients of the droplet with the solid walls. The model was validated by comparing the droplet neck evolution at the T-junction where breakup occurs. The wetting conditions utilized for comparison were regulated to be as close as possible to those reported by Hoang et al [82]. The dimensionless neck thickness was plotted with

the dimensionless time and compared with the corresponding cases of three capillary numbers $\mathrm{Ca}=0.0029,0.0063$, and 0.0078 respectively. Good agreement was observed 
for most of the evolution except for the fast rupture stage.

\subsection{Conclusions}

\subsection{Miscible Mixture Conclusions}

1. The distribution of miscible mixtures is highly affected by the surface forces. The highest impact was noticed near the walls while the inner regions tend to stay close to the initial state unless the channel height is comparable to the intermolecular force spacial range.

2. Surfaces with selective affinity that are hydrophobic to one fluid and hydrophilic to the other are the best choice for the pre-concentration of water-ethanol mixtures.

3. The use of uneven wetting conditions offered the highest density difference for both components.

4. Modifying the cross interaction parameter enhanced the dispersion which in turn increases the separation efficiency.

5. Modifying the height of the channel either by changing the relaxation times or altering the number of lattices in y-direction had a greater effect with uneven wetting conditions.

6. The separation efficiency is increased by adding solid posts with a patterned spacing downstream.

7. Increasing the density of the posts raised the separation efficiency. However, the pressure drop increased $125 \%$ and the flow rate is decreased $200 \%$ when the number of posts columns is increased from 3 to 30 . 
8. The LBM for miscible mixtures is more stable than equivalent simulations of multiphase flow due to the absence of spurious currents and density waves.

\subsection{Two-Phase Flow Conclusions}

1. Uneven wetting conditions on two-phase flow in microchannels were achieved by modifying the upper section contact angle.

2. Asymmetry in wetting conditions produces an interfacial force directed towards the higher wettability region (section of smaller contact angle) with a magnitude proportional to the difference between contact angles.

3. Droplets driven by a carrier fluid in a T-junction can be directed to a preferential side by manipulating the interaction between the fluid and the confining solid surfaces.

4. Without droplet breakup, higher separation efficiencies may be achieved by a successive sequence of T-junctions.

5. With a contact angle difference $130^{\circ}$ between the upper and lower surfaces, no droplet breakup is observed for the entire range of capillary numbers and droplet lengths, tested. This yields a potential high separation efficiency.

6. The viscosity ratio $\lambda$ does not have a significant influence controlling breakup and non-breakup regimes because of the competing effects.

7. The geometrical parameters such as main channel aspect ratio and the side to main channel width ratio have a noticeable impact on the breakup and nonbreakup regimes. 


\subsection{Future Work}

In this work, we developed a multi-component LBM Model capable of computationally studying flows of immiscible and miscible fluids. The results presented represent only initial application of this model. With the now viable LBM model a number of multicomponent flows may be studied. These include but are not limited to:

1. Investigate the corners effect on miscible mixtures. Expanding the miscible case to a 3 dimensional simulation using LBM.

2. Introducing a bifurcation to the straight channel studied in this work in the miscible case to determine separation efficiency with two outlets of different wetting conditions.

3. Studying both the miscible and immiscible cases experimentally by fabricating channels from materials of selective affinity like carbon nano-tubes, palladium, and zeolite.

4. Using channel of Y-shape outlets and investigating the effect of changing the angle between outlets on breakup and non-breakup regimes for two-phase flow under uneven wetting conditions.

5. Using a T-shaped channel with side channels at an angle $\alpha$ from the vertical and analyzing the effect of $\alpha$ on the separation of two-phase flow. See Figure 6.1. 


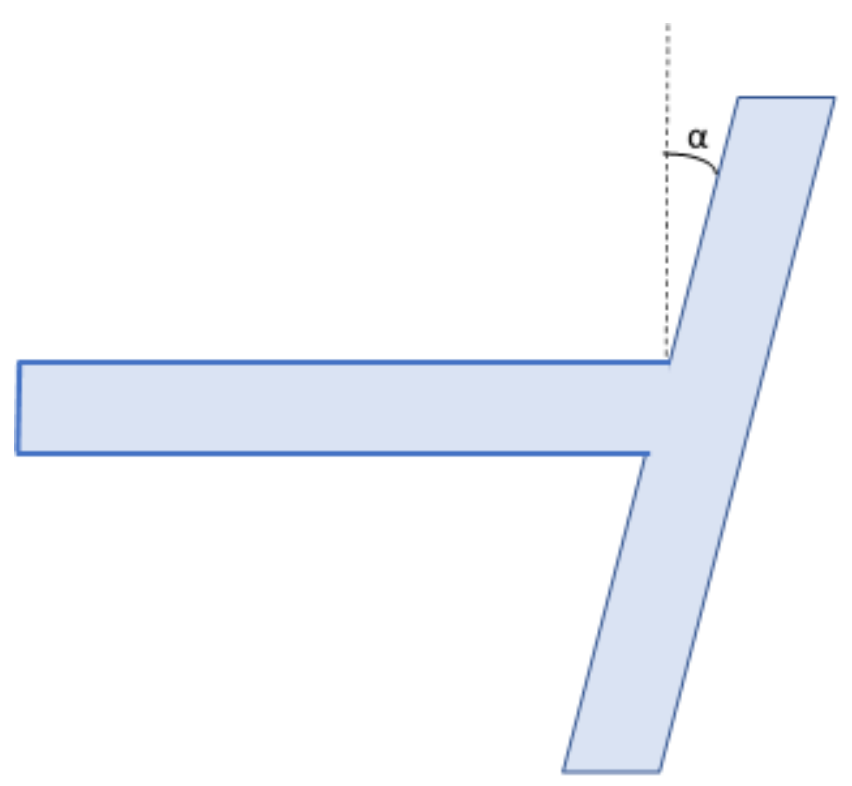

Figure 6.1: A suggested T-junction with tilted side channels to reduce the difference in wetting conditions required for two-phase flow separation.

6. Employing uneven conditions to separate bubbly and dispersed flows instead of slug flow.

7. Exploring the surface force displacement of a fluid by a second fluid in a microchannel.

8. Identifying interaction coefficients, and cross interaction parameters for selected fluid pairs under static experimental conditions then testing these coefficient under flow conditions to determine the universality of the interaction coefficients with the cross interaction parameters.

9. Studying droplet coalesce in unconfined and confined geometries.

10. Simulating and investigating digital microfluidics which depends inherently on controlling fluid-surface interactions. 
11. Expanding the multidimensional to include potential homogeneous or heterogeneous reactions between species. 


\section{Bibliography}

[1] A. Gavriilidis and J. E. A. Shaw, "Separation units," Microchemical Engineering in Practice, pp. 131-163, 2010.

[2] R. D. Noble and R. Agrawal, "Separations research needs for the 21st century," Industrial \& engineering chemistry research, vol. 44, no. 9, pp. 2887-2892, 2005.

[3] J. D. Seader, E. J. Henley, and D. K. Roper, "Separation process principles," 1998.

[4] R.-J. Yang, C.-C. Liu, Y.-N. Wang, H.-H. Hou, and L.-M. Fu, "A comprehensive review of micro-distillation methods," Chemical Engineering Journal, 2016.

[5] S. Gravelle, H. Yoshida, L. Joly, C. Ybert, and L. Bocquet, "Carbon membranes for efficient water-ethanol separation," The Journal of chemical physics, vol. 145, no. 12 , p. $124708,2016$.

[6] J. Coronas and J. Santamaria, "The use of zeolite films in small-scale and microscale applications," Chemical engineering science, vol. 59, no. 22-23, pp. 48794885, 2004.

[7] H. D. Tong, F. Gielens, J. G. Gardeniers, H. V. Jansen, J. W. Berenschot, M. J. de Boer, J. De Boer, C. J. van Rijn, and M. C. Elwenspoek, "Microsieve supporting palladium-silver alloy membrane and application to hydrogen separation," Journal of microelectromechanical systems, vol. 14, no. 1, pp. 113-124, 2005 .

[8] P. K. Panigrahi, Transport Phenomena in Microfluidic Systems. John Wiley \& Sons, 2016.

[9] S. Colin, Microfluidics. John Wiley \& Sons, 2013.

[10] P. Tabeling, Introduction to microfluidics. Oxford University Press on Demand, 2005.

[11] N.-T. Nguyen, S. T. Wereley, and S. A. M. Shaegh, Fundamentals and applications of microfluidics. Artech house, 2019. 
[12] T. M. Squires and S. R. Quake, "Microfluidics: Fluid physics at the nanoliter scale," Reviews of modern physics, vol. 77, no. 3, p. 977, 2005.

[13] C. Kleinstreuer, Microfluidics and nanofluidics: theory and selected applications. John Wiley \& Sons, 2013.

[14] J. N. Israelachvili, Intermolecular and surface forces. Academic press, 2015.

[15] E. Kolehmainen and I. Turunen, "Micro-scale liquid-liquid separation in a platetype coalescer," Chemical Engineering and Processing: Process Intensification, vol. 46, no. 9, pp. 834-839, 2007.

[16] J. G. Kralj, "Continuous flow separation techniques for microchemical synthesis," Ph.D. dissertation, Massachusetts Institute of Technology, 2005.

[17] N. Assmann, A. Ładosz, and P. Rudolf von Rohr, "Continuous micro liquid-liquid extraction," Chemical Engineering \& Technology, vol. 36, no. 6, pp. 921-936, 2013.

[18] K. F. Lam, E. Sorensen, and A. Gavriilidis, "Review on gas-liquid separations in microchannel devices," Chemical Engineering Research and Design, vol. 91, no. 10, pp. 1941-1953, 2013.

[19] T. T. Huang, N. S. Mosier, and M. R. Ladisch, "Surface engineering of microchannel walls for protein separation and directed microfluidic flow." Journal of separation science, vol. 29, no. 12, pp. 1733-42, Aug 2006.

[20] D. H. Yoon, J. B. Ha, Y. K. Bahk, T. Arakawa, S. Shoji, and J. S. Go, "Sizeselective separation of micro beads by utilizing secondary flow in a curved rectangular microchannel." Lab on a chip, vol. 9, no. 1, pp. 87-90, Jan 2009.

[21] J. F. Edd, D. Di Carlo, K. J. Humphry, S. Köster, D. Irimia, D. A. Weitz, and M. Toner, "Controlled encapsulation of single-cells into monodisperse picolitre drops," Lab on a Chip, vol. 8, no. 8, pp. 1262-1264, 2008.

[22] J.-P. Matas, V. Glezer, É. Guazzelli, and J. F. Morris, "Trains of particles in finite-reynolds-number pipe flow," Physics of Fluids, vol. 16, no. 11, pp. 41924195, 2004.

[23] T. Inamuro, K. Maeba, and F. Ogino, "Flow between parallel walls containing the lines of neutrally buoyant circular cylinders," International Journal of Multiphase Flow, vol. 26, no. 12, pp. 1981-2004, 2000.

[24] Y. Yan, J. F. Morris, and J. Koplik, "Hydrodynamic interaction of two particles in confined linear shear flow at finite reynolds number," Physics of fluids, vol. 19, no. 11, p. 113305, 2007. 
[25] A. A. S. Bhagat, S. S. Kuntaegowdanahalli, and I. Papautsky, "Continuous particle separation in spiral microchannels using dean flows and differential migration," Lab on a Chip, vol. 8, no. 11, pp. 1906-1914, 2008.

[26] F. Tian, W. Zhang, L. Cai, S. Li, G. Hu, Y. Cong, C. Liu, T. Li, and J. Sun, "Microfluidic co-flow of newtonian and viscoelastic fluids for high-resolution separation of microparticles," Lab on a Chip, 2017.

[27] N. Oozeki, S. Ookawara, K. Ogawa, P. Loeb, and V. Hessel, "Characterization of microseparator/classifier with a simple arc microchannel," AIChE journal, vol. 55, no. 1, pp. 24-34, 2009.

[28] Z. Wu, B. Willing, J. Bjerketorp, J. K. Jansson, and K. Hjort, "Soft inertial microfluidics for high throughput separation of bacteria from human blood cells." Lab on a chip, vol. 9, no. 9, pp. 1193-9, May 2009.

[29] D. Di Carlo, "Inertial microfluidics." Lab on a chip, vol. 9, no. 21, pp. 3038-46, Nov 2009.

[30] J. Yue, G. Chen, Q. Yuan, L. Luo, and Y. Gonthier, "Hydrodynamics and mass transfer characteristics in gas-liquid flow through a rectangular microchannel," Chemical Engineering Science, vol. 62, no. 7, pp. 2096-2108, 2007.

[31] V. Hessel, P. Löb, K. Mevissen, F. Schönfeld et al., "Pseudo 3-d simulation of a falling film microreactor based on realistic channel and film profiles," Chemical Engineering Science, vol. 63, no. 21, pp. 5149-5159, 2008.

[32] A. Constantinou and A. Gavriilidis, "Co2 absorption in a microstructured mesh reactor," Industrial 83 Engineering Chemistry Research, vol. 49, no. 3, pp. 10411049, 2009.

[33] N. Shao, A. Gavriilidis, and P. Angeli, "Mass transfer during taylor flow in microchannels with and without chemical reaction," Chemical Engineering Journal, vol. 160, no. 3, pp. 873-881, 2010.

[34] M. Zanfir, X. Sun, and A. Gavriilidis, "Microstructured mesh contactor for asymmetric transfer hydrogenation with simultaneous stripping: modeling and experiments," Industrial \& Engineering Chemistry Research, vol. 47, no. 23, pp. 8995-9005, 2008.

[35] P. Chasanis, K. Kehrmann, J. Kern, R. Zecirovic, M. Grünewald, and E. Kenig, "Investigation of a microstructured high efficiency contactor," Chemical Engineering and Processing: Process Intensification, vol. 50, no. 11, pp. 1244-1251, 2011. 
[36] P. Moschou, M. De Croon, J. Van Der Schaaf, and J. Schouten, "Nitrogen stripping of isopropyl-alcohol and toluene in a falling film micro reactor: Gas side mass transfer experiments and modelling at isothermal conditions," Chemical engineering science, vol. 76, pp. 216-223, 2012.

[37] A. Constantinou, F. Ghiotto, K. F. Lam, and A. Gavriilidis, "Stripping of acetone from water with microfabricated and membrane gas-liquid contactors." The Analyst, vol. 139, no. 1, pp. 266-72, Jan 2014.

[38] O. K. Castell, C. J. Allender, and D. A. Barrow, "Liquid-liquid phase separation: characterisation of a novel device capable of separating particle carrying multiphase flows," Lab on a Chip, vol. 9, no. 3, pp. 388-396, 2009.

[39] F. Scheiff, M. Mendorf, D. Agar, N. Reis, and M. Mackley, "The separation of immiscible liquid slugs within plastic microchannels using a metallic hydrophilic sidestream." Lab on a chip, vol. 11, no. 6, pp. 1022-9, Mar 2011.

[40] D. Peroni, W. van Egmond, W. T. Kok, and H.-G. Janssen, "Advancing liquid/liquid extraction through a novel microfluidic device: Theory, instrumentation and applications in gas chromatography," Journal of Chromatography A, vol. 1226, pp. 77-86, 2012.

[41] W. Gaakeer, M. De Croon, J. Van Der Schaaf, and J. Schouten, "Liquid-liquid slug flow separation in a slit shaped micro device," Chemical engineering journal, vol. 207, pp. 440-444, 2012.

[42] K. Zhao and D. Li, "Manipulation and separation of oil droplets by using asymmetric nano-orifice induced de dielectrophoretic method," Journal of colloid and interface science, vol. 512, pp. 389-397, 2018.

[43] T. Krüger, H. Kusumaatmaja, A. Kuzmin, O. Shardt, G. Silva, and E. M. Viggen, The Lattice Boltzmann Method. Springer, 2017.

[44] G. P. Zank, "Transport processes in space physics and astrophysics," 2014.

[45] A. A. Mohamad, Lattice Boltzmann method: fundamentals and engineering applications with computer codes. Springer Science \& Business Media, 2011.

[46] R. D. Present, Kinetic theory of gases. McGraw-Hill, 1958, vol. 222.

[47] M. Portinari, "2d and 3d verification an validation of the lattice boltzmann method," Ph.D. dissertation, École Polytechnique de Montréal, 2015.

[48] Z. Guo and C. Shu, Lattice Boltzmann method and its applications in engineering. World Scientific, 2013, vol. 3. 
[49] Y. Qian, D. d'Humières, and P. Lallemand, "Lattice bgk models for navier-stokes equation," Europhysics Letters, vol. 17, no. 6, p. 479, 1992.

[50] P. Bhatnagar, E. Gross, and M. Krook, "A model for collision processes in gases. i. small amplitude processes in charged and neutral one-component systems," Physical Review, vol. 94, no. 3, p. 511, 1954.

[51] A. White and C. Chong, "Rotational invariance in the three-dimensional lattice boltzmann method is dependent on the choice of lattice," Journal of Computational Physics, vol. 230, no. 16, pp. 6367-6378, 2011.

[52] G. Silva and V. Semiao, "Truncation errors and the rotational invariance of three-dimensional lattice models in the lattice boltzmann method," Journal of Computational Physics, vol. 269, pp. 259-279, 2014.

[53] X. He and L.-S. Luo, "Theory of the lattice boltzmann method: From the boltzmann equation to the lattice boltzmann equation," Physical Review E, vol. 56, no. 6 , p. $6811,1997$.

[54] J. Latt, "Hydrodynamic limit of lattice boltzmann equations," Ph.D. dissertation, University of Geneva, 2007.

[55] G. McNamara and G. Zanetti, "Use of the boltzmann equation to simulate lattice-gas automata," Physical Review Letters, vol. 61, no. 20, pp. 2332-2335, 1988 .

[56] A. M. Aguirre Mesa, A. M. A. Mesa et al., "Study of lattice boltzmann boundary conditions for fluid structure interaction," Master's thesis, Universidad EAFIT, 2014.

[57] A. Dadvand et al., "Simulation of the motion of two elastic membranes in poiseuille shear flow via a combined immersed boundary-lattice boltzmann method," Journal of Computational Science, vol. 12, pp. 51-61, 2016.

[58] M. Bouzidi, M. Firdaouss, and P. Lallemand, "Momentum transfer of a boltzmann-lattice fluid with boundaries," Physics of fluids, vol. 13, no. 11, pp. 3452-3459, 2001.

[59] D. d'Humières, "Multiple-relaxation-time lattice boltzmann models in three dimensions," Philosophical Transactions of the Royal Society of London A: Mathematical, Physical and Engineering Sciences, vol. 360, no. 1792, pp. 437-451, 2002.

[60] H. Huang, M. C. Sukop, X.-Y. Lu et al., Multiphase lattice Boltzmann methods: Theory and application. Wiley Online Library, 2015. 
[61] I. Orlanski, "A simple boundary condition for unbounded hyperbolic flows," Journal of computational physics, vol. 21, no. 3, pp. 251-269, 1976.

[62] Q. Lou, Z. Guo, and B. Shi, "Evaluation of outflow boundary conditions for twophase lattice boltzmann equation," Physical review E, vol. 87, no. 6, p. 063301, 2013.

[63] X. Shan and H. Chen, "Lattice boltzmann model for simulating flows with multiple phases and components," Physical Review E, vol. 47, no. 3, p. 1815, 1993.

[64] X. Shan and H. Chen(b), "Simulation of nonideal gases and liquid-gas phase transitions by the lattice boltzmann equation," Physical Review E, vol. 49, no. 4, p. 2941, 1994.

[65] M. Sbragaglia, R. Benzi, L. Biferale, S. Succi, K. Sugiyama, and F. Toschi, "Generalized lattice boltzmann method with multirange pseudopotential," Physical Review E, vol. 75, no. 2, p. 026702, 2007.

[66] G. Falcucci, G. Bella, G. Chiatti, S. Chibbaro, M. Sbragaglia, S. Succi et al., "Lattice boltzmann models with mid-range interactions," Commun. Comput. Phys, vol. 2, no. 6, pp. 1071-1084, 2007.

[67] M. E. McCracken and J. Abraham, "Lattice boltzmann methods for binary mixtures with different molecular weights," Physical Review E, vol. 71, no. 4, p. 046704, 2005.

[68] M. B. Schlaffer, "Non-reflecting boundary conditions for the lattice boltzmann method," Ph.D. dissertation, Technische Universität München, 2013.

[69] T. Young, "An essay on the cohesion of fluids," Philosophical Transactions of the Royal Society of London, vol. 95, pp. 65-87, 1805.

[70] P. Rapolu, "Capillarity effect on two-phase flow resistance in microchannels," Master's thesis, University of Cincinnati, 2008.

[71] M. A. Burns, C. H. Mastrangelo, T. S. Sammarco, F. P. Man, J. R. Webster, B. Johnsons, B. Foerster, D. Jones, Y. Fields, A. R. Kaiser et al., "Microfabricated structures for integrated dna analysis," Proceedings of the National Academy of Sciences, vol. 93, no. 11, pp. 5556-5561, 1996.

[72] T. S. Sammarco and M. A. Burns, "Heat-transfer analysis of microfabricated thermocapillary pumping and reaction devices," Journal of Micromechanics and Microengineering, vol. 10, no. 1, p. 42, 2000. 
[73] M. A. Burns, B. N. Johnson, S. N. Brahmasandra, K. Handique, J. R. Webster, M. Krishnan, T. S. Sammarco, P. M. Man, D. Jones, D. Heldsinger, C. H. Mastrangelo, and D. T. Burke, "An integrated nanoliter dna analysis device." Science (New York, N.Y.), vol. 282, no. 5388, pp. 484-7, Oct 1998.

[74] T. S. Sammarco and M. A. Burns, "Thermocapillary pumping of discrete drops in microfabricated analysis devices," AIChE Journal, vol. 45, no. 2, pp. 350-366, 1999.

[75] M. Dhindsa, J. Heikenfeld, S. Kwon, J. Park, P. D. Rack, and I. Papautsky, "Virtual electrowetting channels: electronic liquid transport with continuous channel functionality," Lab on a Chip, vol. 10, no. 7, pp. 832-836, 2010.

[76] A. Banerjee, E. Kreit, Y. Liu, J. Heikenfeld, and I. Papautsky, "Reconfigurable virtual electrowetting channels," Lab on a Chip, vol. 12, no. 4, pp. 758-764, 2012.

[77] D.-G. Lee, J. Park, J. Bae, and H.-Y. Kim, "Dynamics of a microliquid prism actuated by electrowetting," Lab on a Chip, vol. 13, no. 2, pp. 274-279, 2013.

[78] J. H. Bannock, T. W. Phillips, A. M. Nightingale et al., "Microscale separation of immiscible liquids using a porous capillary," Analytical Methods, vol. 5, no. 19, pp. 4991-4998, 2013.

[79] J. Fernandez-Toledano, T. Blake, and J. De Coninck, "Young's equation for a two-liquid system on the nanometer scale," Langmuir, vol. 33, no. 11, pp. 29292938, 2017.

[80] "Imagej." [Online]. Available: https://imagej.nih.gov/ij/

[81] F. M. White, Fluid mechanics. McGraw-hill, 2011.

[82] D. Hoang, L. Portela, C. Kleijn, M. Kreutzer, and V. Van Steijn, "Dynamics of droplet breakup in a t-junction," Journal of Fluid Mechanics, vol. 717, 2013.

[83] A. Rohatgi, "Webplotdigitizer," Apr 2019. [Online]. Available: https://automeris.io/WebPlotDigitizer

[84] "Dortmund data bank." [Online]. Available: http://www.ddbst.com/

[85] M. A. Boles and Y. A. Cengel, Thermodynamics: an engineering approach. McGraw-Hill, 1989.

[86] A. Leshansky, S. Afkhami, M.-C. Jullien, and P. Tabeling, "Obstructed breakup of slender drops in a microfluidic t junction," Physical review letters, vol. 108, no. 26, p. 264502, 2012. 
[87] X. Sun, C. Zhu, T. Fu, Y. Ma, and H. Z. Li, "Dynamics of droplet breakup and formation of satellite droplets in a microfluidic t-junction," Chemical Engineering Science, vol. 188, pp. 158-169, 2018.

[88] M.-C. Jullien, M.-J. Tsang Mui Ching, C. Cohen, L. Menetrier, and P. Tabeling, "Droplet breakup in microfluidic t-junctions at small capillary numbers," Physics of Fluids, vol. 21, no. 7, p. 072001, 2009.

[89] D. Link, S. L. Anna, D. Weitz, and H. Stone, "Geometrically mediated breakup of drops in microfluidic devices," Physical review letters, vol. 92, no. 5, p. 054503, 2004 .

[90] A. M. Leshansky and L. M. Pismen, "Breakup of drops in a microfluidic t junction," Physics of Fluids, vol. 21, no. 2, p. 023303, 2009. 


\section{Appendix A Computer Programs}

\section{A.1 Computational Expense}

Both miscible and immiscible cases are simulated using computer codes we built in Fortran language. The hardware employed to run these codes is a high performance computing cluster (HPC) administered by Portland Institute for Computational Science. Each case was run on a single computational node of 20 cores. Each node has Dual Intel Xeon E2630 v4, 10 cores at $2.2 \mathrm{GHz}$ processor and $128 \mathrm{~GB} 2133 \mathrm{MHz}$ RAM. The miscible case, since it is a 2D problem with D2Q9 lattice, was run on a single core sequentially. The immiscible case was run on 20 cores due to the intensive need for parallel computing. The maximum usage of RAM was $1.1 \mathrm{~GB}$ for the miscible case and $8.5 \mathrm{~GB}$ for the immiscible one. The time required to run the simulation $10^{5}$ iterations was 9 minutes for the miscible case with $44 \times 600$ mesh size and 6 hours for the immiscible case with $250 \times 32 \times 360$ mesh size. 


\section{A.2 Miscible Case Flowchart}

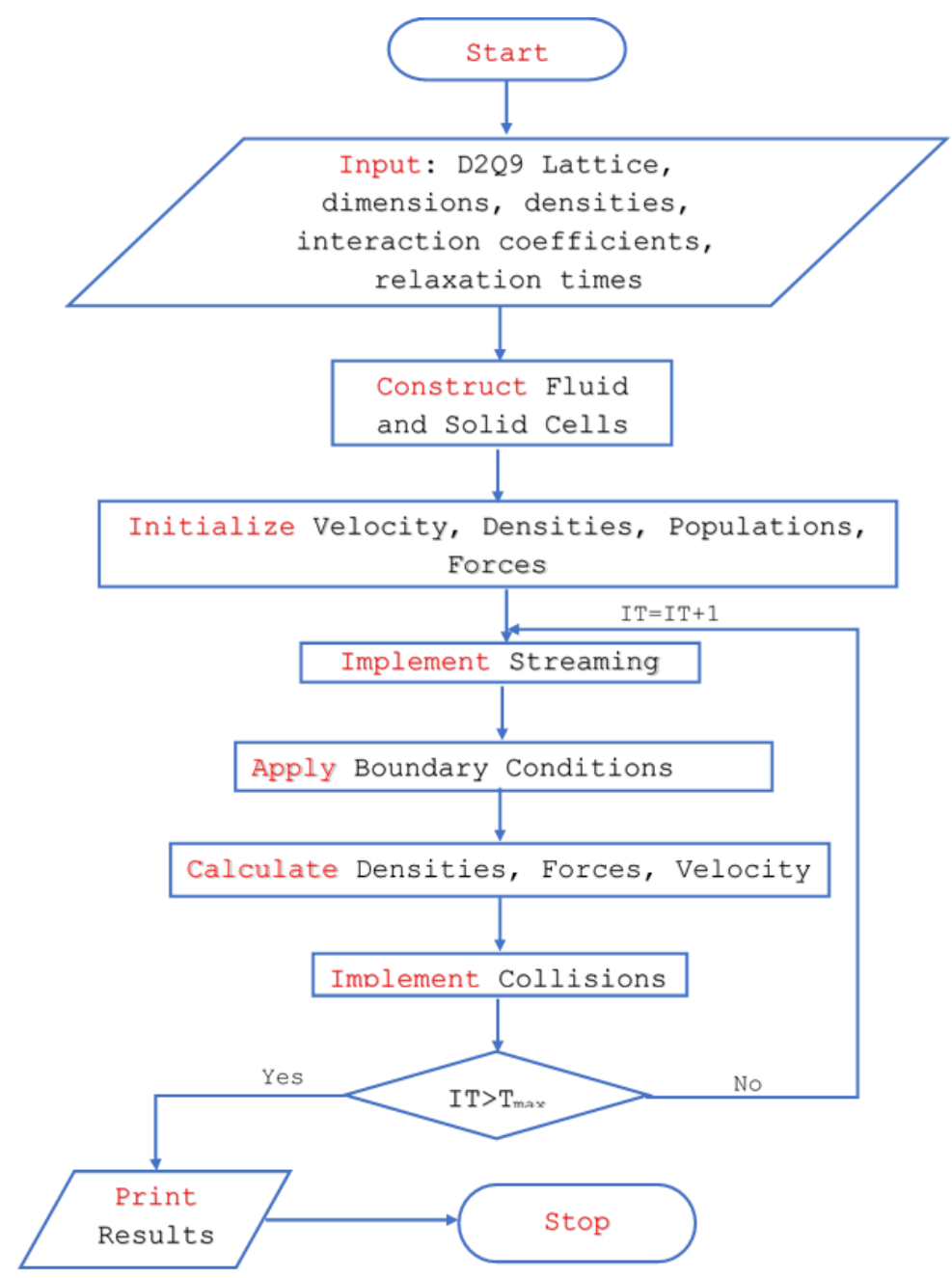

Figure A.2.1: Flowchart of the multicomponent multirange D2Q9 lattice Boltzmann method for a binary mixture in microchannel.

\section{A.3 Miscible Case Code}

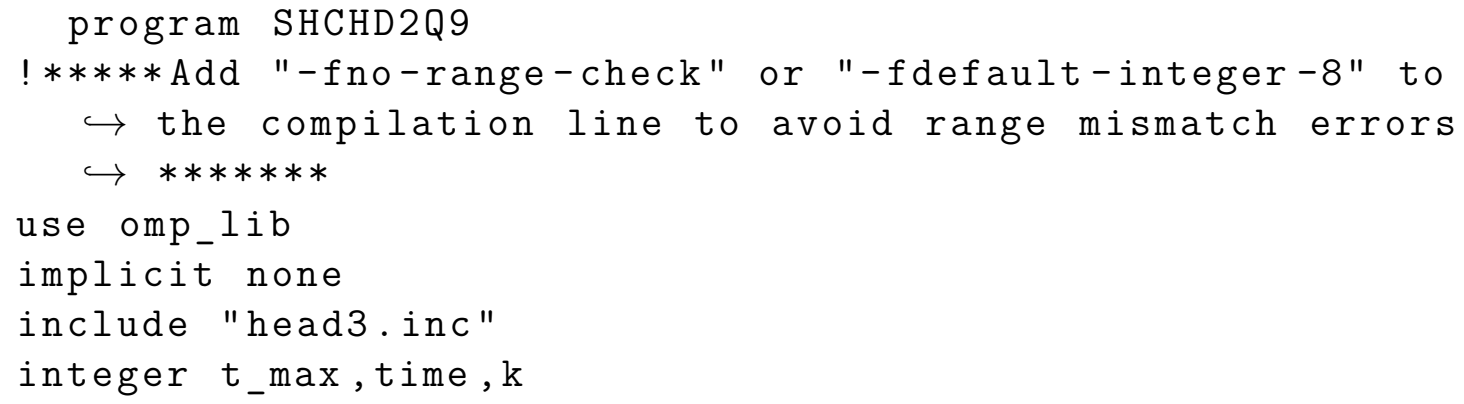


INTEGER, DIMENSION $(:,:)$, ALLOCATABLE : : obst

REAL, DIMENSION $(:,:)$, ALLOCATABLE : : u_x, u_y, rho, Gai, Gbi, Fx

$\hookrightarrow$,Fy, Fxa, Fxb, Fya, Fyb,Fza, Fzb

REAL, DIMENSION $(:,:,:)$, ALLOCATABLE : fa_old,fb_old,fa,fb

REAL, DIMENSION $(:,:)$, ALLOCATABLE : :Ga_rho, Gb_rho, rho_a,

$\hookrightarrow$ rho_b,psi_a,psi_b, Gaa, Gbb

real d_in

ALLOCATE (obst ( $1 x, 1 y), u_{-} x(1 x, 1 y), u_{-} y(1 x, 1 y), r h o(1 x, 1 y)$, Gai (

$\hookrightarrow 1 \mathrm{x}, 1 \mathrm{y}), \mathrm{Gbi}(1 \mathrm{x}, 1 \mathrm{y}), \mathrm{Fx}(1 \mathrm{x}, 1 \mathrm{y}), \quad$ \&

Fy $(1 x, 1 y), F x a(1 x, 1 y), F x b(1 x, 1 y), F y a(l x, l y), F y b(l x, l y), F z a($

$\hookrightarrow 1 \mathrm{x}, 1 \mathrm{y}), \mathrm{Fzb}(1 \mathrm{x}, 1 \mathrm{y}))$

ALLOCATE ( $f a_{-} o l d(0: 8,1 x, 1 y), f b_{-} o l d(0: 8,1 x, 1 y), f a(0: 8,1 x, 1 y)$

$\hookrightarrow, \mathrm{fb}(0: 8,1 \mathrm{x}, 1 \mathrm{y}))$

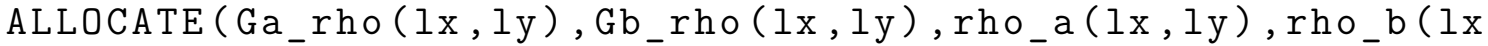

$\hookrightarrow, 1 \mathrm{y}), \mathrm{psi} a(1 \mathrm{x}, 1 \mathrm{y})$, \&

psi_b(lx, ly), Gaa (lx, ly), Gbb(lx, ly))

! Velocity vectors of the D3Q19 velocity model, $x c, y c, z c$ $\hookrightarrow$ are

! the $x, y, z$ components of the vector, respectively. The $\hookrightarrow$ order of

! the velocity vector is slightly different from that in $\hookrightarrow$ Fig.1.1.

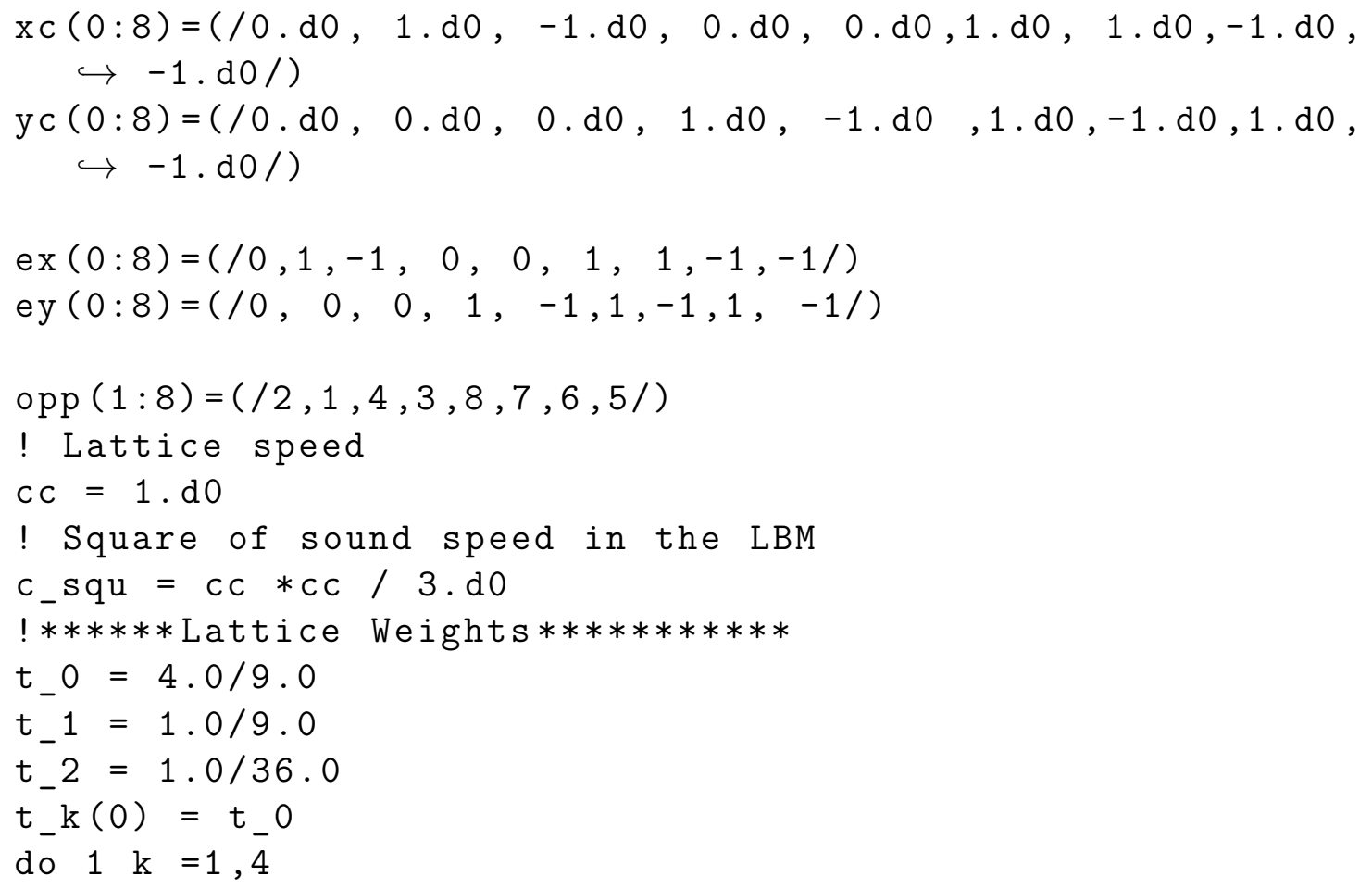




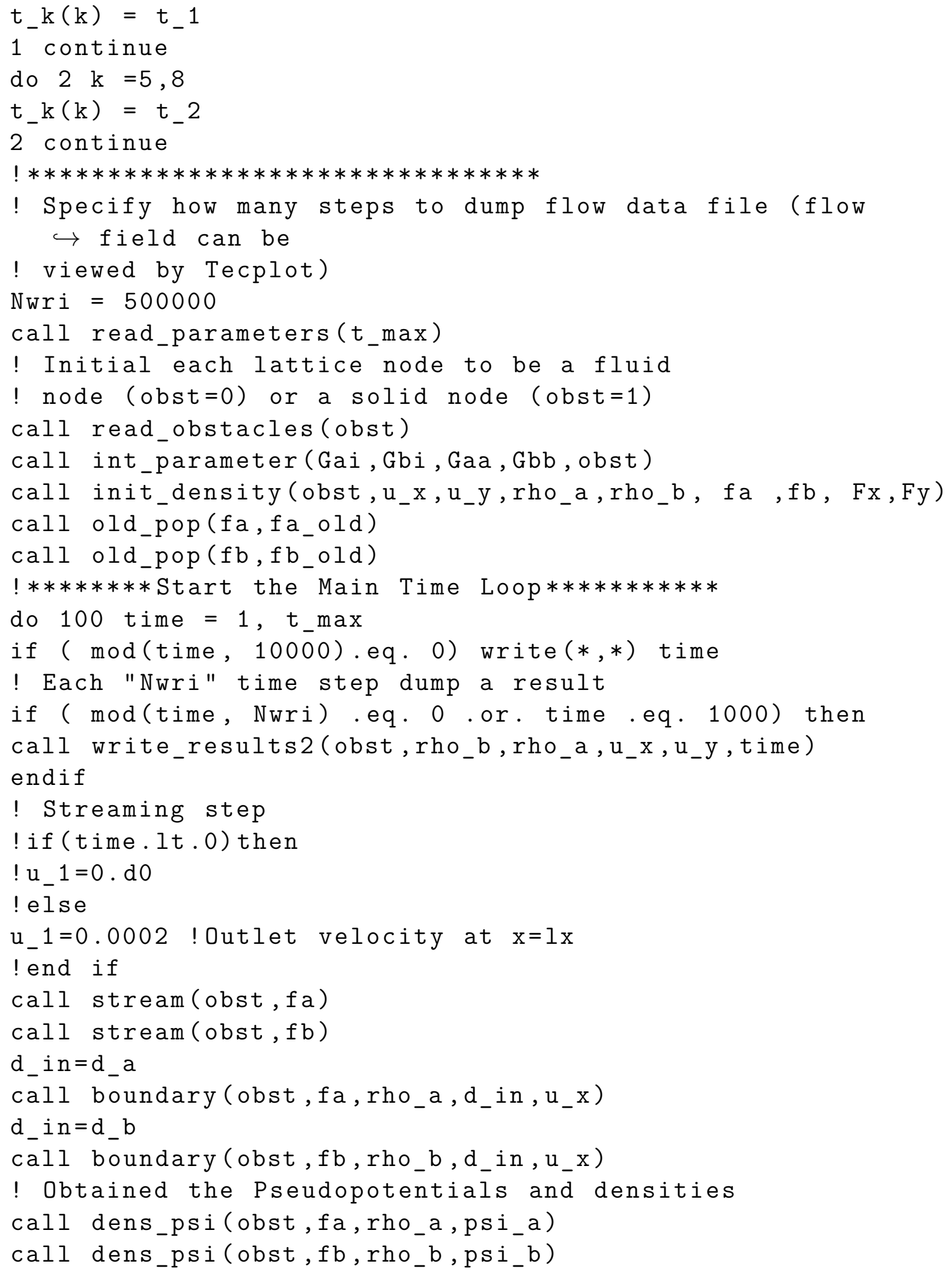


call int_force(obst,psi_a,psi_b, Gai, Gbi, Gaa, Gbb, Fx, Fy, Fxa , $\hookrightarrow$ Fxb,Fya,Fyb)

call get_uvw(obst, u_x, u_y, fa, fb, Fx, Fy, rho_a, rho_b)

call old_pop(fa,fa_old)

call old_pop(fb,fb_old)

call collision(obst, u_x, u_y, rho_a, rho_b, fa, fb, Fxa, Fya, Fxb , $\hookrightarrow$ Fyb )

100 continue

! $* * * * * * * * * * * * * * * * * * * * * * * * * * * * * * * * * * * * * * * *$

call write_results2 (obst, rho_b, rho_a, u_x, u_y, time)

end program SHCHD2Q9

subroutine read_parameters (t_max)

implicit none

include "head3. inc"

! Interaction Parameter which controls the repulsion

$\hookrightarrow$ strength

integer t_max

Real Re1, nu_a, nu_b, Cs_a, Cs_b, $x_{-} a, x_{-} b, c_{-} m, n u_{-} m, t a u_{-} m, d t$, $\hookrightarrow \mathrm{mu} u_{-} \mathrm{s}, \mathrm{den} \mathrm{n}_{-}$

$t_{-\max }=10000000$

$\mathrm{G} 12=1.0 \quad ! 1.0$

$\mathrm{Ga}_{-} \mathrm{a}=0.0 !-0.25$

$\mathrm{Gb} \mathrm{b}_{-} \mathrm{b}=0.0$

$\mathrm{Ga}_{-} \mathrm{s}=1.0 \quad ! 1.0$

$\mathrm{Gb}_{-} \mathrm{s}=-1.0 \quad !-1.0$

$\mathrm{d}_{-} \mathrm{a}=1.0$

$\mathrm{d}_{-} \mathrm{b}=0.784 \quad ! \quad 0.784$

$d_{-} s=1.0$

tau_a $=0.75$

tau_b $=0.75$

$\mathrm{nu} \mathrm{a}_{-}=8.941 \mathrm{e}-7 \quad$ !water kinematic viscosity [m²/s]

$\mathrm{nu} \mathrm{b}_{-}=1.386 \mathrm{e}-6$ ! ethanol kinematic viscosity [m²/s]

$\mathrm{Cs}_{-} \mathrm{a}=1500.0 \quad$ !water speed of sound $[\mathrm{m} / \mathrm{s}]$

$\mathrm{Cs}_{-} \mathrm{b}=1142.29$ ! ethanol speed of sound [m/s]

$x_{-} a=d_{-} a /\left(d_{-} a+d_{-} b\right) \quad$ !mass fraction of fluid A

$x_{-} b=d_{-} b /\left(d_{-} a+d_{-} b\right) \quad$ !mass fraction of fluid $B$

$\mathrm{Cs} \mathrm{s}_{-}=\mathrm{x}_{-} \mathrm{a} * \mathrm{Cs} \mathrm{s}_{-} \mathrm{a}+\mathrm{x}_{-} \mathrm{b} * \mathrm{Cs}_{-} \mathrm{b}$ ! Mixture speed of Sound

$n u_{-} m=x_{-} a * n u_{-} a+x_{-} b * n u_{-} b \quad$ ! Mixture Viscosity

tau_m $=x_{-} a * t a u_{-} a+x_{-} b * t a u_{-} b$ ! Mixture Relaxation Time

$\mathrm{dt}=\mathrm{nu} \mathrm{u}_{-} \mathrm{m} /\left(\mathrm{Cs}_{-} \mathrm{m} * \mathrm{Cs}_{-} \mathrm{m} *\left(\mathrm{tau} \mathrm{u}_{-}-0.5\right)\right) \quad$ ! Time Step [Second]

$d x=d t * C_{s} m * \operatorname{sqrt}(3.0)$

! Space Step [meter]

$d e n_{-} m=\left(d_{-} a+d_{-} b\right)$ 


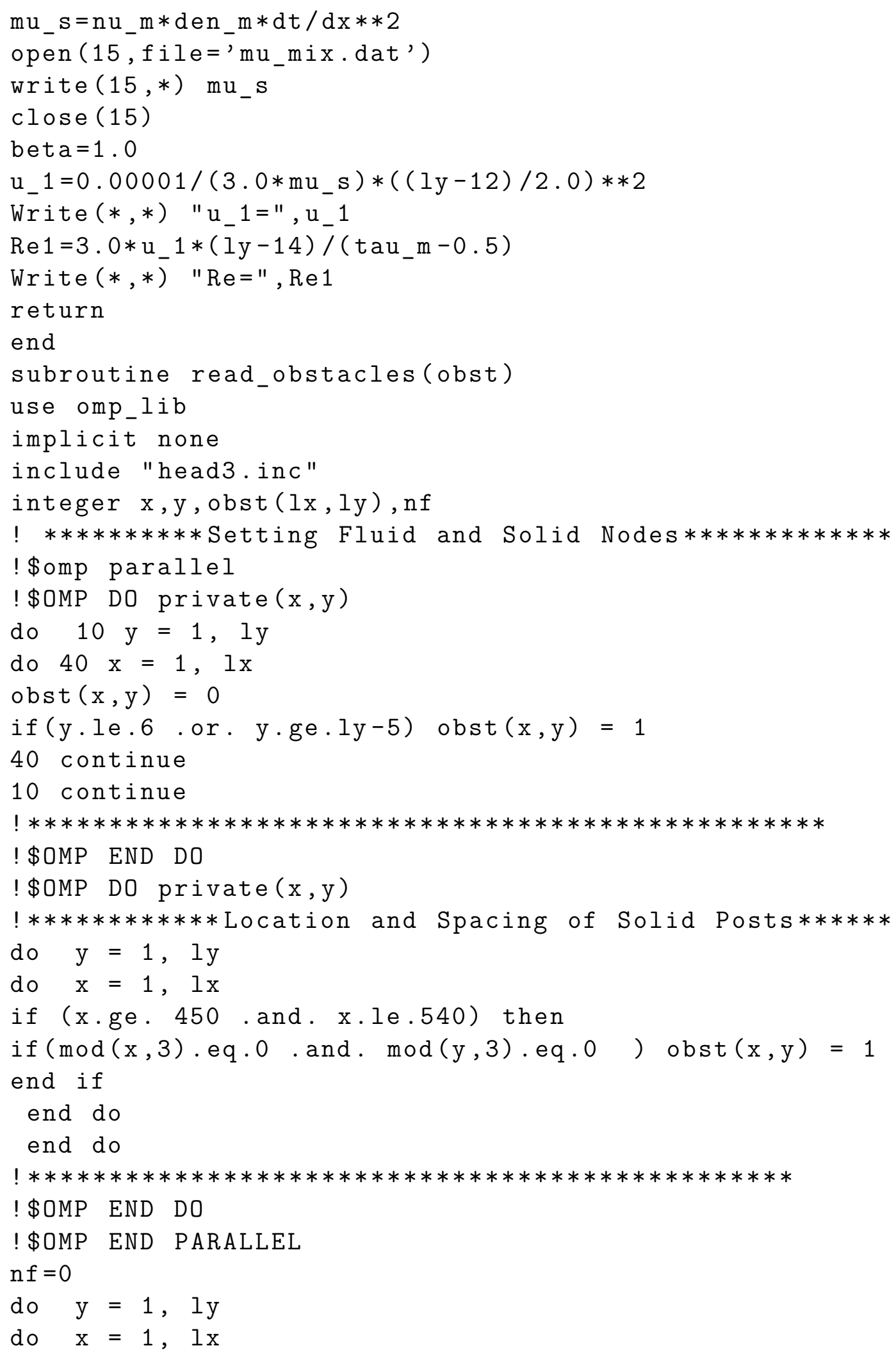




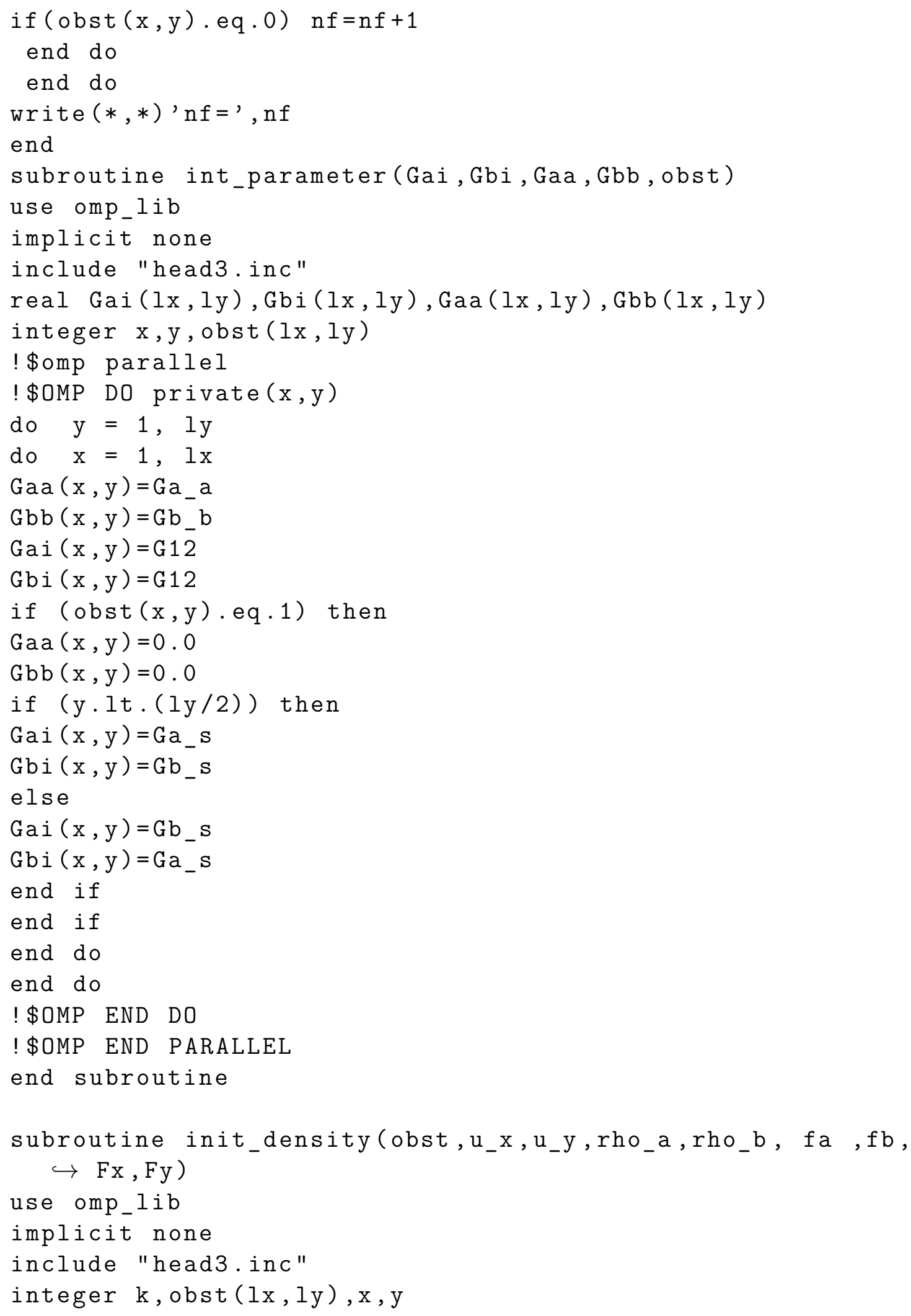




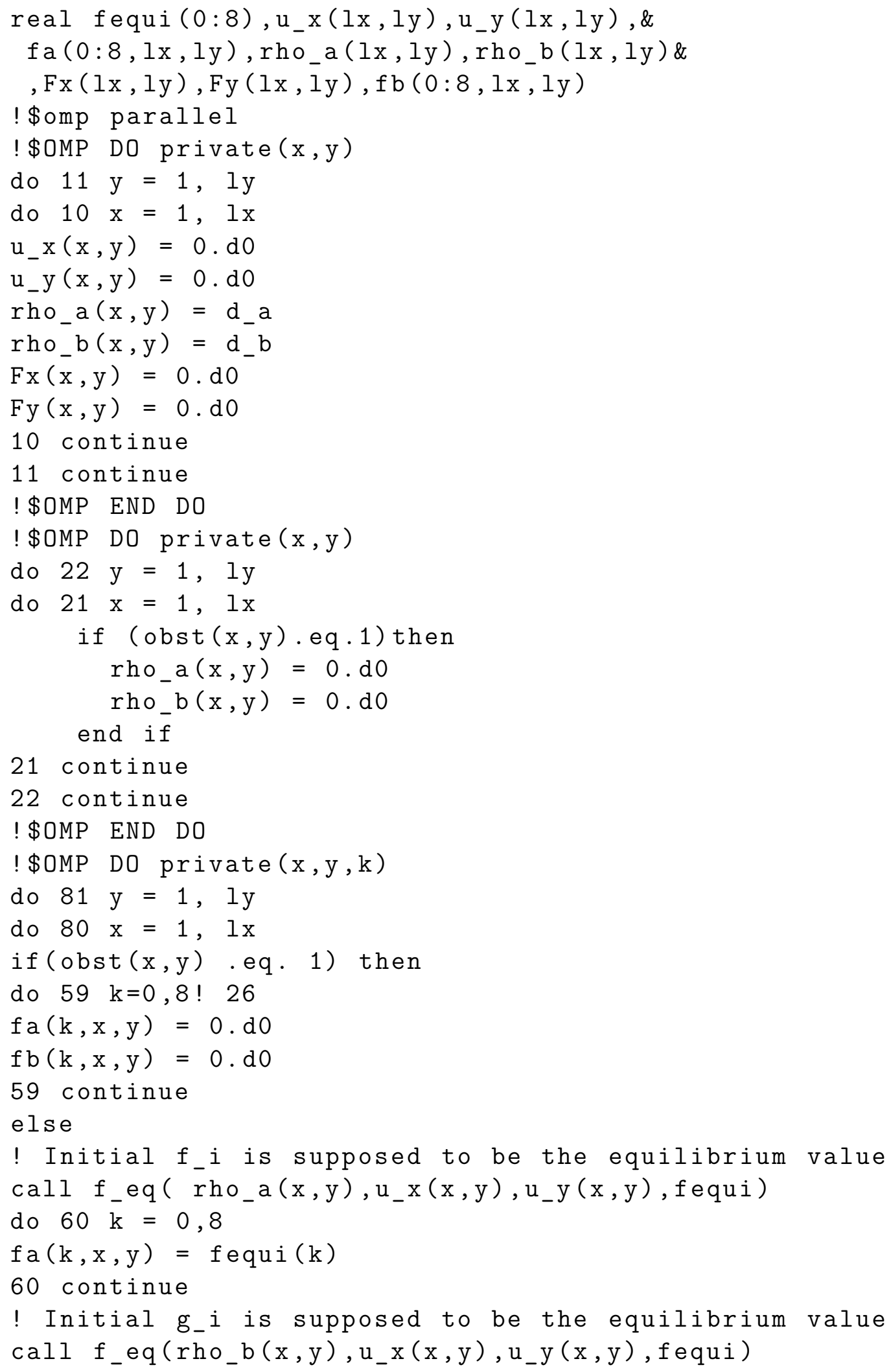




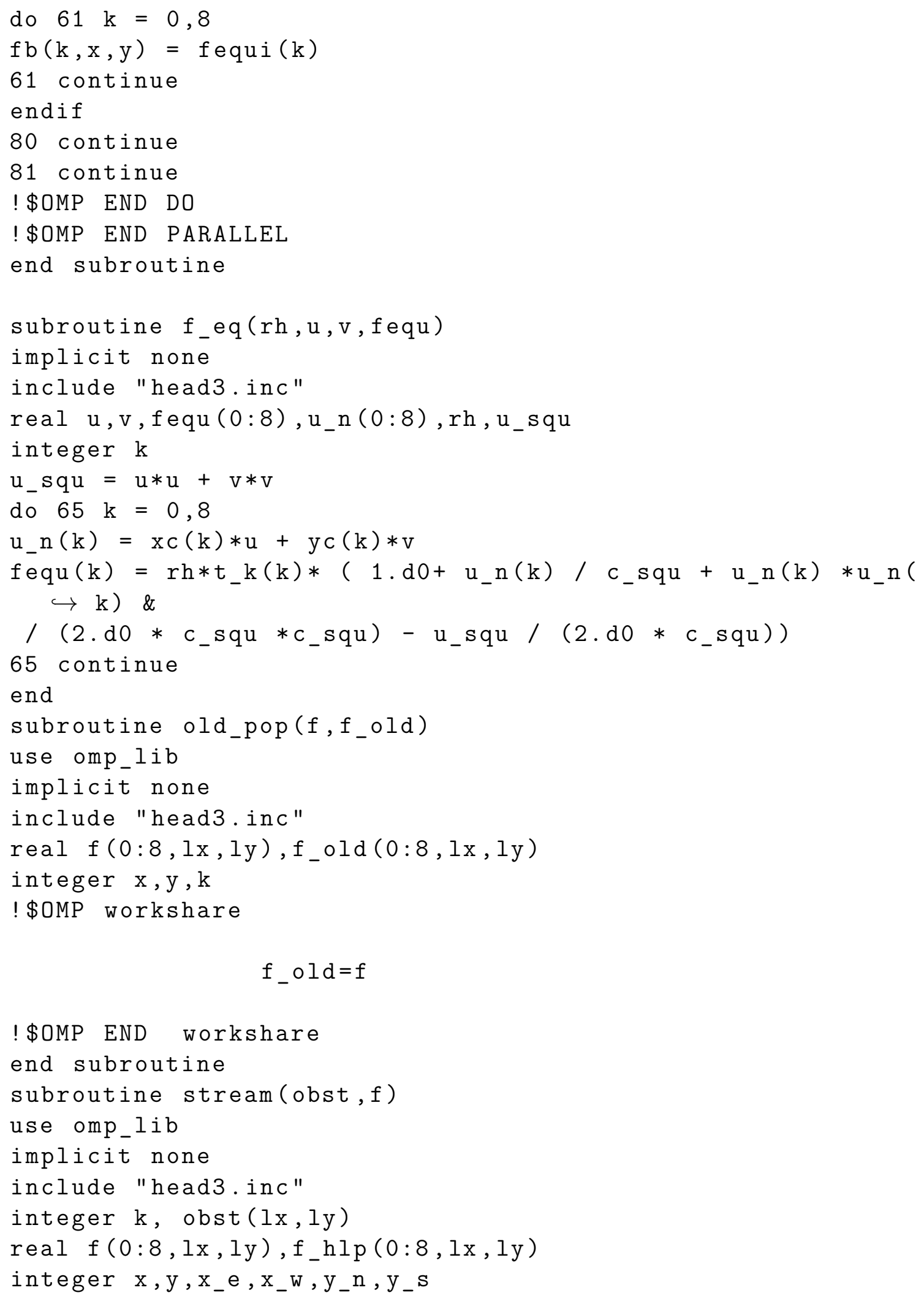

$$
f \_o l d=f
$$

! \$OMP END workshare

end subroutine 


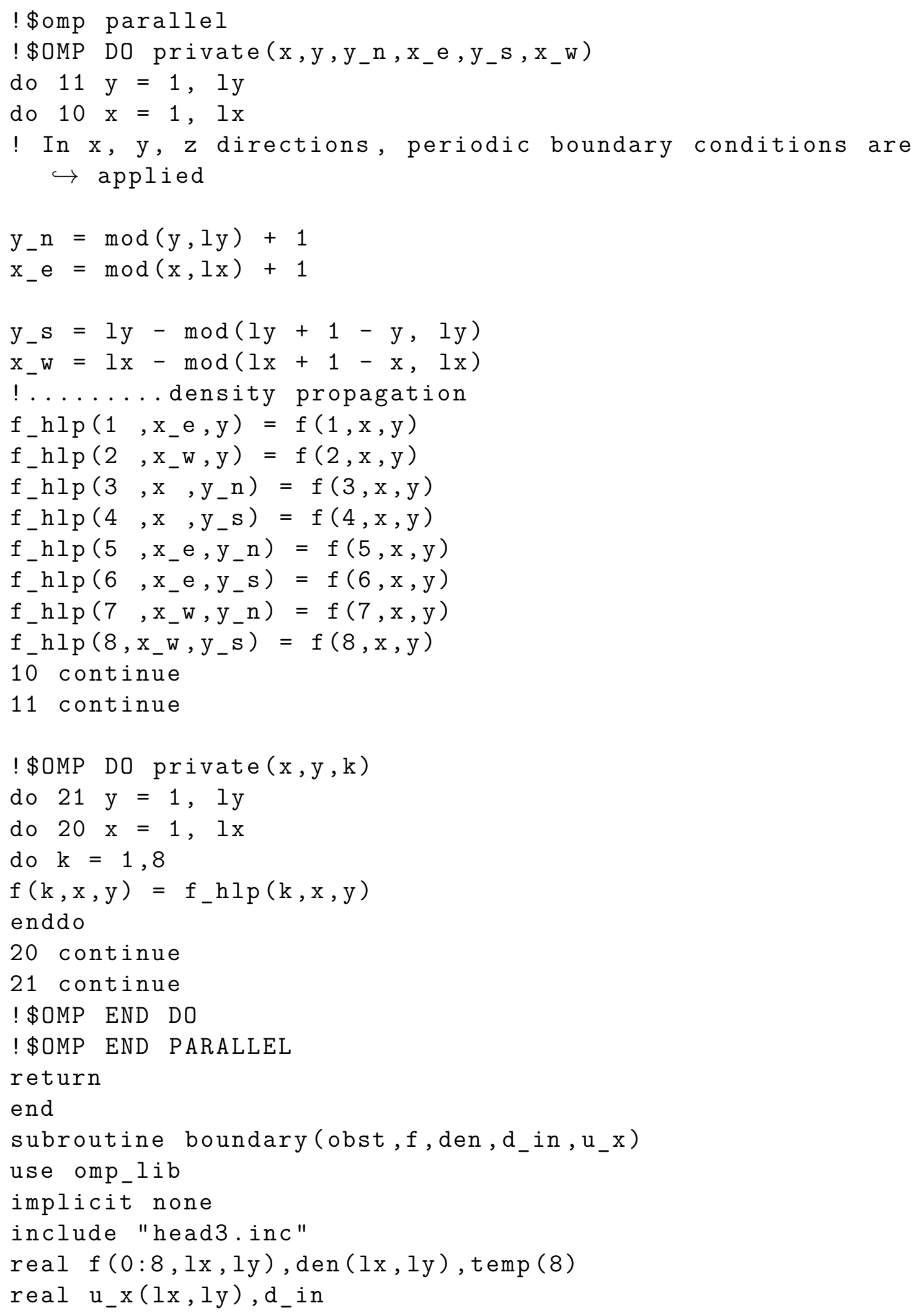




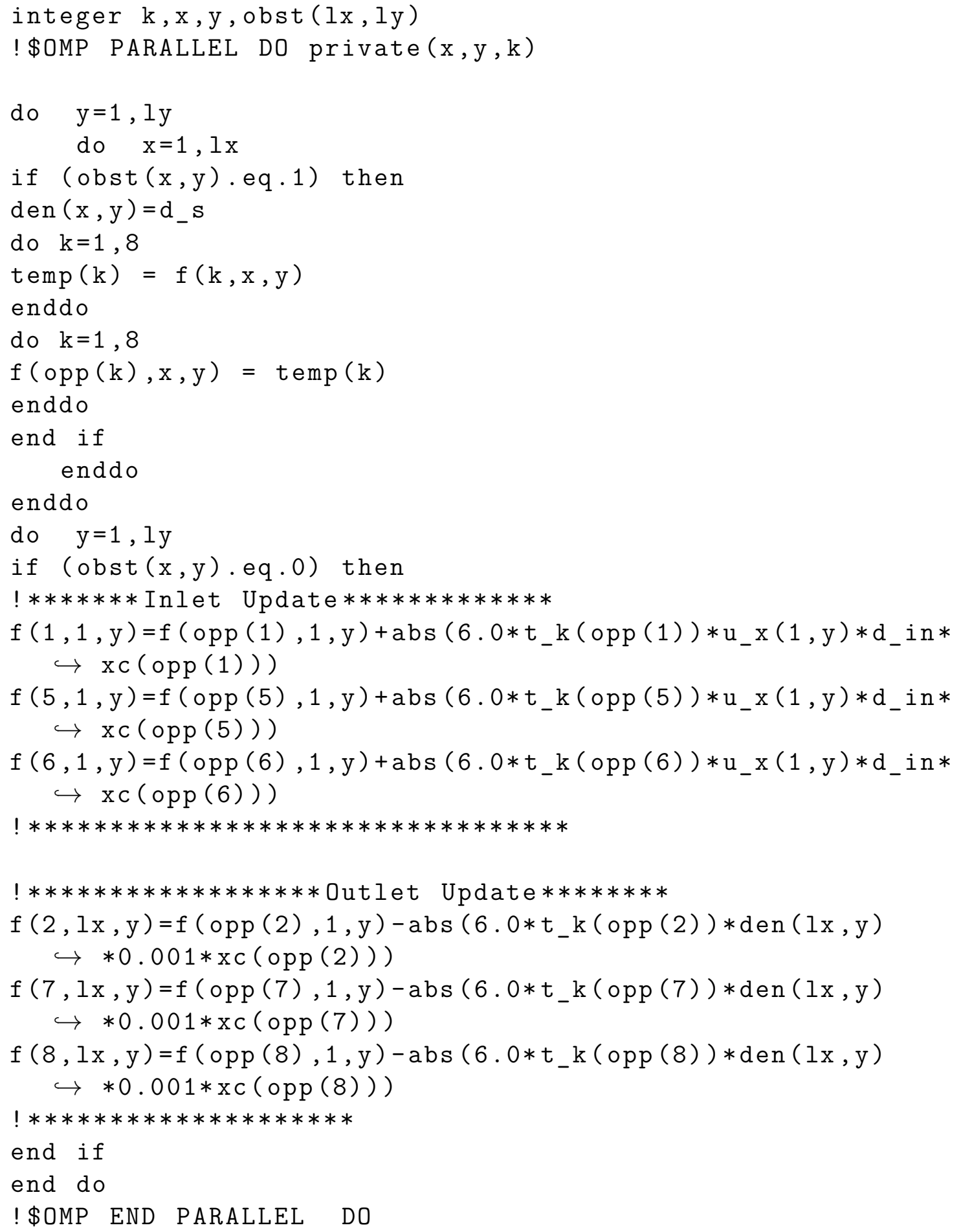

! Apply the Half-way Bounce Back on the first inlet with u $\hookrightarrow=\mathrm{u} 1$

! this is used to update f5 f11 f12 f15 f 17 at $Z=1$ 


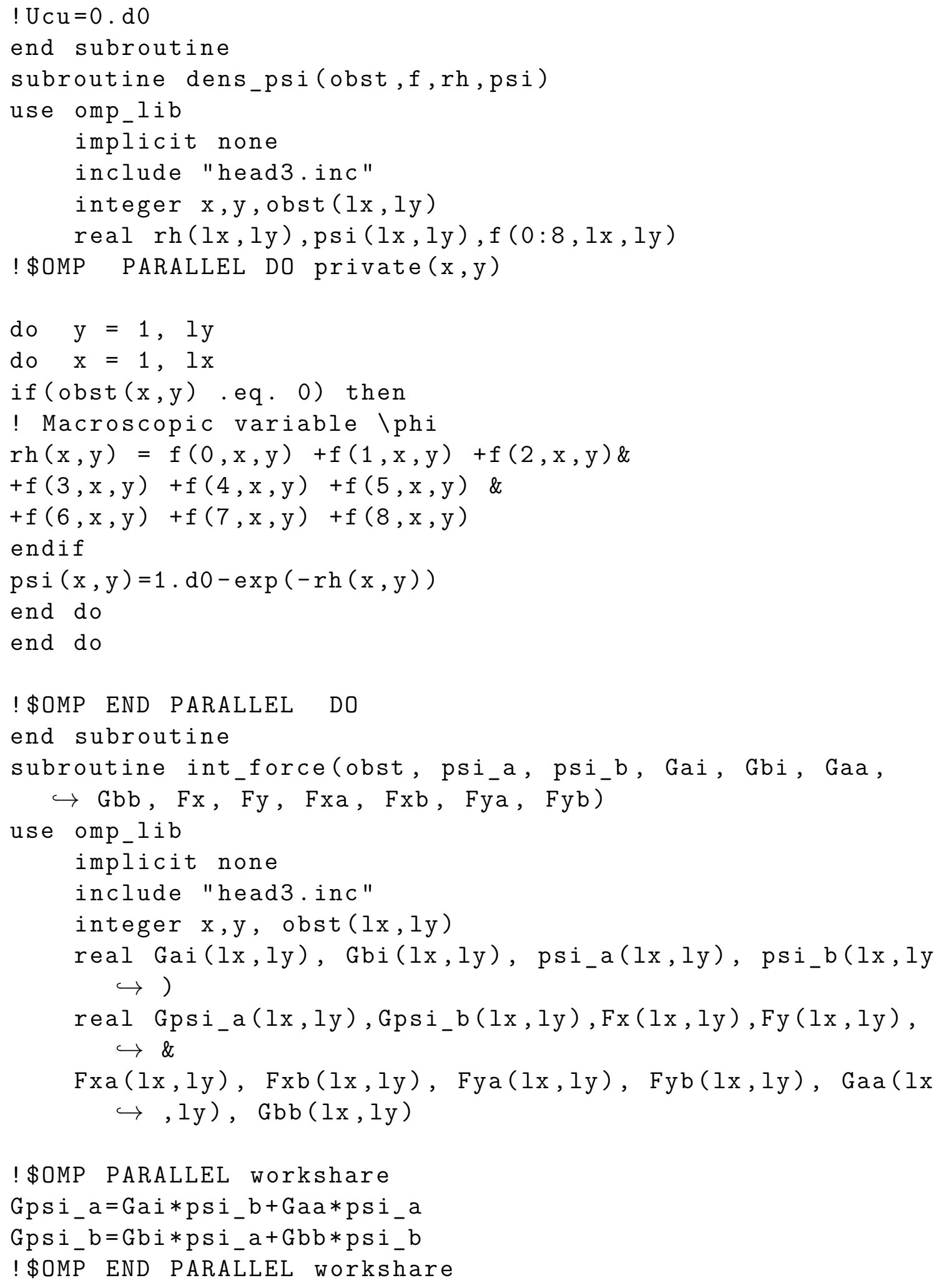


call FxFyFz(obst,psi_a, Gpsi_a,Fxa,Fya)

call FxFyFz(obst,psi_b,Gpsi_b, Fxb,Fyb)

! \$OMP PARALLEL workshare

$$
\begin{aligned}
& F x=F x a+F x b \\
& F y=F y a+F y b
\end{aligned}
$$

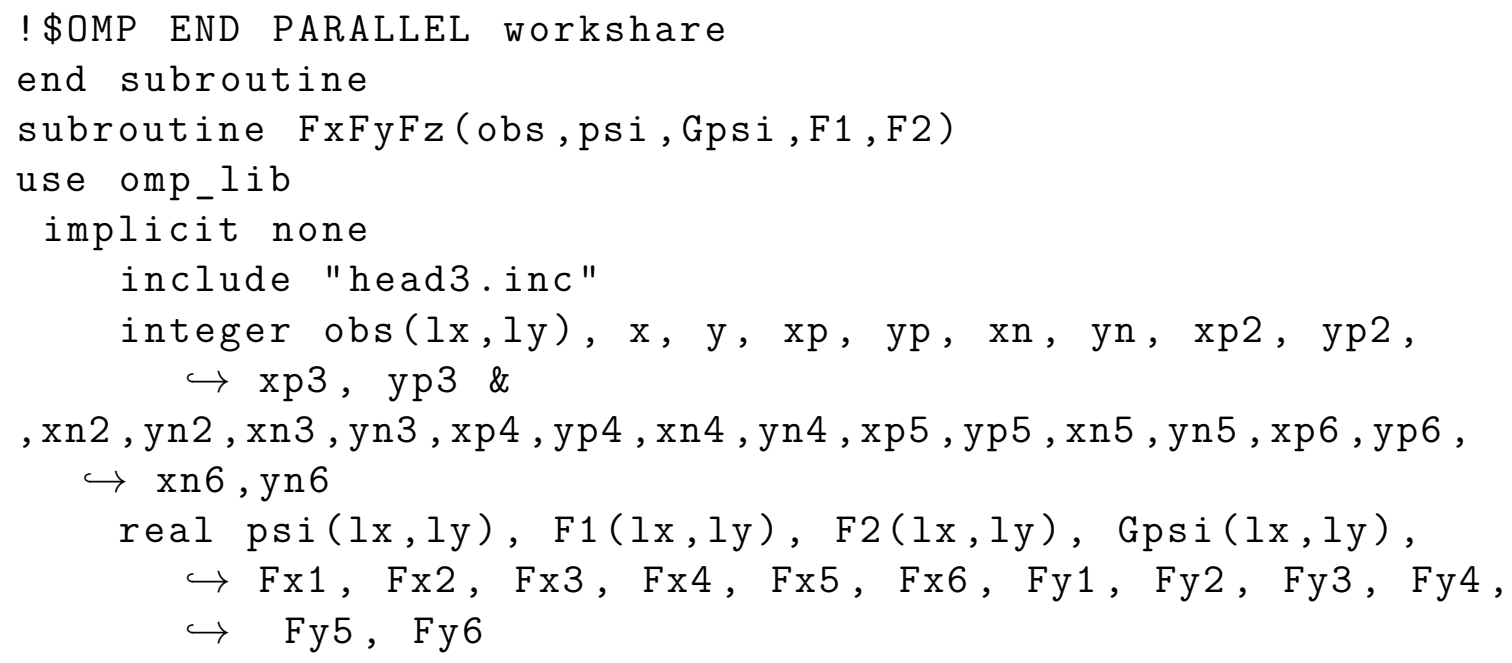

! \$OMP PARALLEL D0 private (x, y, xp, xp2, xp3, xp4, xp5,

$\hookrightarrow \mathrm{xp} 6, \mathrm{xn}, \mathrm{xn} 2, \mathrm{xn} 3, \mathrm{xn} 4, \mathrm{xn} 5, \mathrm{xn} 6, \mathrm{yp}, \mathrm{yp} 2, \mathrm{yp} 3, \mathrm{yp} 4$,

$\hookrightarrow$ yp5, yp6, yn, yn2, yn3, yn4, yn5, yn6)

$$
\begin{aligned}
& \text { do } y=1,1 y \\
& \text { do } x=1,1 x
\end{aligned}
$$

$\mathrm{F} 1(\mathrm{x}, \mathrm{y})=0 . \mathrm{d} 0$

$\mathrm{F} 2(\mathrm{x}, \mathrm{y})=0 . \mathrm{d} 0$

if (x.gt. 50 . and. x.lt.550) then

$$
\begin{aligned}
& \text { xp }=x+1 \\
& y p=y+1 \\
& x n=x-1 \\
& y n=y-1 \\
& \text { xp2 }=x+2 \\
& \text { yp2 }=y+2 \\
& \text { xn2 }=x-2 \\
& \text { yn2 } 2 y-2
\end{aligned}
$$




$$
\begin{aligned}
\text { xp3 } & =x+3 \\
\text { yp3 } & =y+3 \\
\text { xn3 } & =x-3 \\
\text { yn3 } & =y-3 \\
\text { xp4 } & =x+4 \\
\text { yp4 } & =y+4 \\
\text { xn4 } & =x-4 \\
\text { yn4 } & =y-4 \\
\text { xp5 } & =x+5 \\
\text { yp5 } & =y+5 \\
\text { xn5 } & =x-5 \\
\text { yn5 } & =y-5 \\
\text { xp6 } & =x+6 \\
\text { yp6 } & =y+6 \\
\text { xn6 } & =x-6 \\
\text { yn6 } & =y-6
\end{aligned}
$$

! Periodic boundary is applied here

$$
\begin{aligned}
& \text { if }(\mathrm{xp} \cdot \mathrm{gt} \cdot \mathrm{lx}) \mathrm{xp}=1 \\
& \text { if (xn.lt.1) } x n=1 x \\
& \text { if }(x p 2 \cdot g t \cdot l x) x p 2=x p 2-1 x \\
& \text { if }(\mathrm{xn} 2.1 \mathrm{t} .1) \mathrm{xn} 2=1 \mathrm{x}+\mathrm{xn} 2+1 \\
& \text { if }(x p 3 \cdot g t \cdot 1 x) \operatorname{xp} 3=x p 3-1 x \\
& \text { if (xn3.1t.1) } x n 3=1 x+x n 3+1 \\
& \text { if }(\mathrm{xp} 4 . \mathrm{gt} . \mathrm{lx}) \mathrm{xp} 4=\mathrm{xp} 4-1 \mathrm{x} \\
& \text { if }(\mathrm{xn} 4.1 \mathrm{t} .1) \mathrm{xn} 4=1 \mathrm{x}+\mathrm{xn} 4+1 \\
& \text { if }(\operatorname{xp} 5 \cdot g t \cdot 1 x) \operatorname{xp} 5=x p 5-1 x \\
& \text { if (xn5.1t.1) } \mathrm{xn} 5=1 \mathrm{x}+\mathrm{xn} 5+1 \\
& \text { if }(\mathrm{xp} 6 . \mathrm{gt} \cdot 1 \mathrm{x}) \mathrm{xp} 6=\mathrm{xp} 6-1 \mathrm{x} \\
& \text { if }(\mathrm{xn} 6.1 \mathrm{t} .1) \mathrm{xn} 6=1 \mathrm{x}+\mathrm{xn} 6+1
\end{aligned}
$$

if $($ obs $(x, y) . e q .0)$ then

$$
\begin{aligned}
& \text { Fxi } 1(\operatorname{Gpsi}(x p, y)-G p s i(x n, y)) *(285860656.0 / 3979934595)+\& \\
& (\operatorname{Gpsi}(x p, y p)-G p s i(x n, y p)+G p s i(x p, y n)-G p s i(x n, y n)) \\
& \hookrightarrow *(2113732952.0 / 43779280545) \\
& \text { Fy } 1=(\operatorname{Gpsi}(x, y p)-G p s i(x, y n)) *(285860656 / 3979934595)+\&
\end{aligned}
$$


(Gpsi (xp,yp)-Gpsi (xp,yn) + Gpsi (xn,yp) -Gpsi (xn,yn))

$\hookrightarrow *(2113732952.0 / 43779280545)$

$\mathrm{Fx} 2=(\operatorname{Gpsi}(\operatorname{xp} 2, \mathrm{y})-\operatorname{Gpsi}(\mathrm{xn} 2, \mathrm{y})) *(940787801.0 / 43779280545)+$ $\hookrightarrow$ \&

(Gpsi (xp2,yp)-Gpsi (xn2,yp)+Gpsi (xp2,yn)-Gpsi (xn2,yn) + \& Gpsi (xp,yp2)-Gpsi (xn,yp2)+Gpsi (xp,yn2)-Gpsi (xn,yn2))

$\hookrightarrow *(124525000.0 / 8755856109)+\&$

(Gpsi (xp2,yp2)-Gpsi (xn2,yp2)+Gpsi (xp2,yn2)-Gpsi (xn2,yn2))

$\hookrightarrow *(15841927.0 / 3979934595)$

Fy $2=(\operatorname{Gpsi}(x, y p 2)-\operatorname{Gpsi}(x, y n 2)) *(940787801 / 43779280545)+\&$

(Gpsi (xp,yp2)-Gpsi (xp,yn2)+Gpsi (xn,yp2)-Gpsi (xn,yn2) + \&

$\operatorname{Gpsi}(x p 2, y p)-\operatorname{Gpsi}(x p 2, y n)+\operatorname{Gpsi}(x n 2, y p)-\operatorname{Gpsi}(x n 2, y n))$

$\hookrightarrow *(124525000.0 / 8755856109)+\&$

(Gpsi (xp2,yp2)-Gpsi (xp2,yn2)+Gpsi (xn2,yp2)-Gpsi(xn2,yn2))

$\hookrightarrow *(15841927.0 / 3979934595)$

Fx3 $=(\operatorname{Gpsi}(x p 3, y)-\operatorname{Gpsi}(x n 3, y)) *(2046152.0 / 795986919)+\&$

(Gpsi (xp3,yp)-Gpsi (xn3,yp) +Gpsi (xp3,yn)-Gpsi (xn3,yn) +\&

Gpsi (xp, yp3)-Gpsi (xn,yp3)+Gpsi (xp,yn3)-Gpsi (xn,yn3))

$\hookrightarrow *(14436304.0 / 8755856109)$

Fy3 $=(\operatorname{Gpsi}(x, y p 3)-\operatorname{Gpsi}(x, y n 3)) *(2046152 / 795986919)+\&$

(Gpsi (xp,yp3)-Gpsi (xp,yn3)+Gpsi (xn,yp3)-Gpsi (xn,yn3)+\&

Gpsi (xp3,yp)-Gpsi (xp3,yn)+Gpsi (xn3,yp)-Gpsi (xn3,yn))

$\hookrightarrow *(14436304.0 / 8755856109)$

Fx4 $13537939 *(\operatorname{Gpsi}(\operatorname{xp} 4, y)-\operatorname{Gpsi}(\mathrm{xn} 4, \mathrm{y})) / 140093697744+\&$ $231568 *(\operatorname{Gpsi}(\mathrm{xp} 4, y p)-\mathrm{Gpsi}(\mathrm{xn} 4, \mathrm{yp})+\mathrm{Gpsi}(\mathrm{xp} 4, y n)-\operatorname{Gpsi}(\mathrm{xn} 4$, $\hookrightarrow \mathrm{yn})+\&$

Gpsi (xp,yp4)-Gpsi (xn,yp4)+Gpsi(xp,yn4)-Gpsi (xn,yn4))

$\hookrightarrow / 3979934595+\&$

$18185828 *(\mathrm{Gpsi}(\mathrm{xp} 3, \mathrm{yp} 2)-\mathrm{Gpsi}(\mathrm{xn} 3$, yp2) +Gpsi (xp2,yp3)- Gpsi(

$\hookrightarrow \mathrm{xn} 2, \mathrm{yp} 3)+\&$

Gpsi (xp3,yn2)-Gpsi (xn3,yn2)+Gpsi (xp2,yn3)-Gpsi(xn2,yn3))

$\hookrightarrow / 43779280545$

Fy $4=13537939 *(\operatorname{Gpsi}(x, y p 4)-G p s i(x, y n 4)) / 140093697744+\&$

$231568 *(\mathrm{Gpsi}(\mathrm{xp}, \mathrm{yp} 4)-\mathrm{Gpsi}(\mathrm{xp}, \mathrm{yn} 4)+\mathrm{Gpsi}(\mathrm{xn}, \mathrm{yp} 4)-\mathrm{Gpsi}(\mathrm{xn}$,

$\hookrightarrow \mathrm{yn} 4)+\&$

Gpsi (xp4,yp)-Gpsi (xp4,yn) +Gpsi(xn4,yp)-Gpsi(xn4,yn))

$\hookrightarrow / 3979934595+\&$

$18185828 *(\mathrm{Gpsi}(\mathrm{xp} 2, \mathrm{yp} 3)-\mathrm{Gpsi}(\mathrm{xp} 2, \mathrm{yn} 3)+\mathrm{Gpsi}(\mathrm{xp} 3, \mathrm{yp} 2)-\&$ 
Gpsi (xp3,yn2)+Gpsi (xn2,yp3)-Gpsi (xn2,yn3)+Gpsi (xn3, yp2)$\hookrightarrow \operatorname{Gpsi}(\mathrm{xn} 3, \mathrm{yn} 2)) / 43779280545$

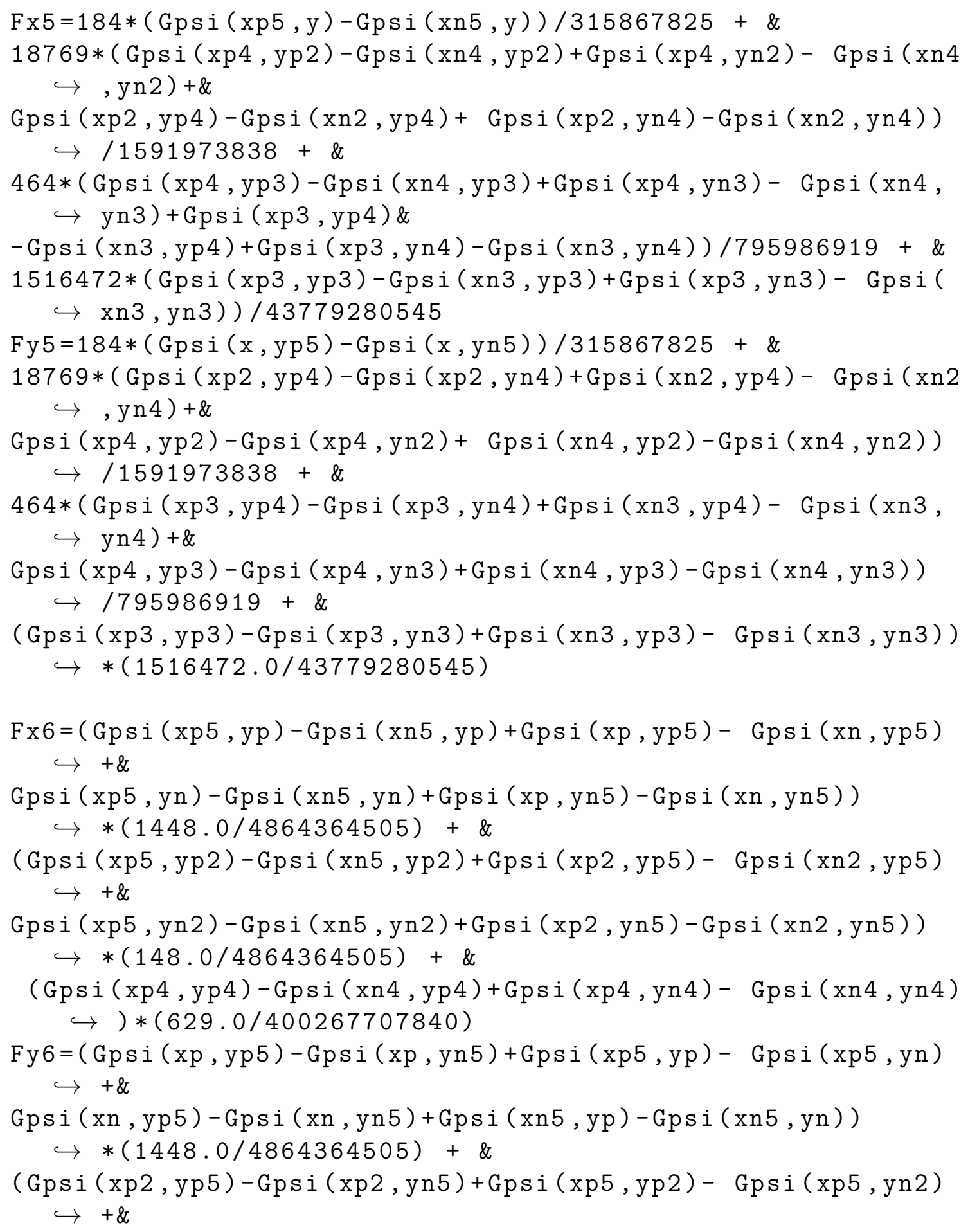




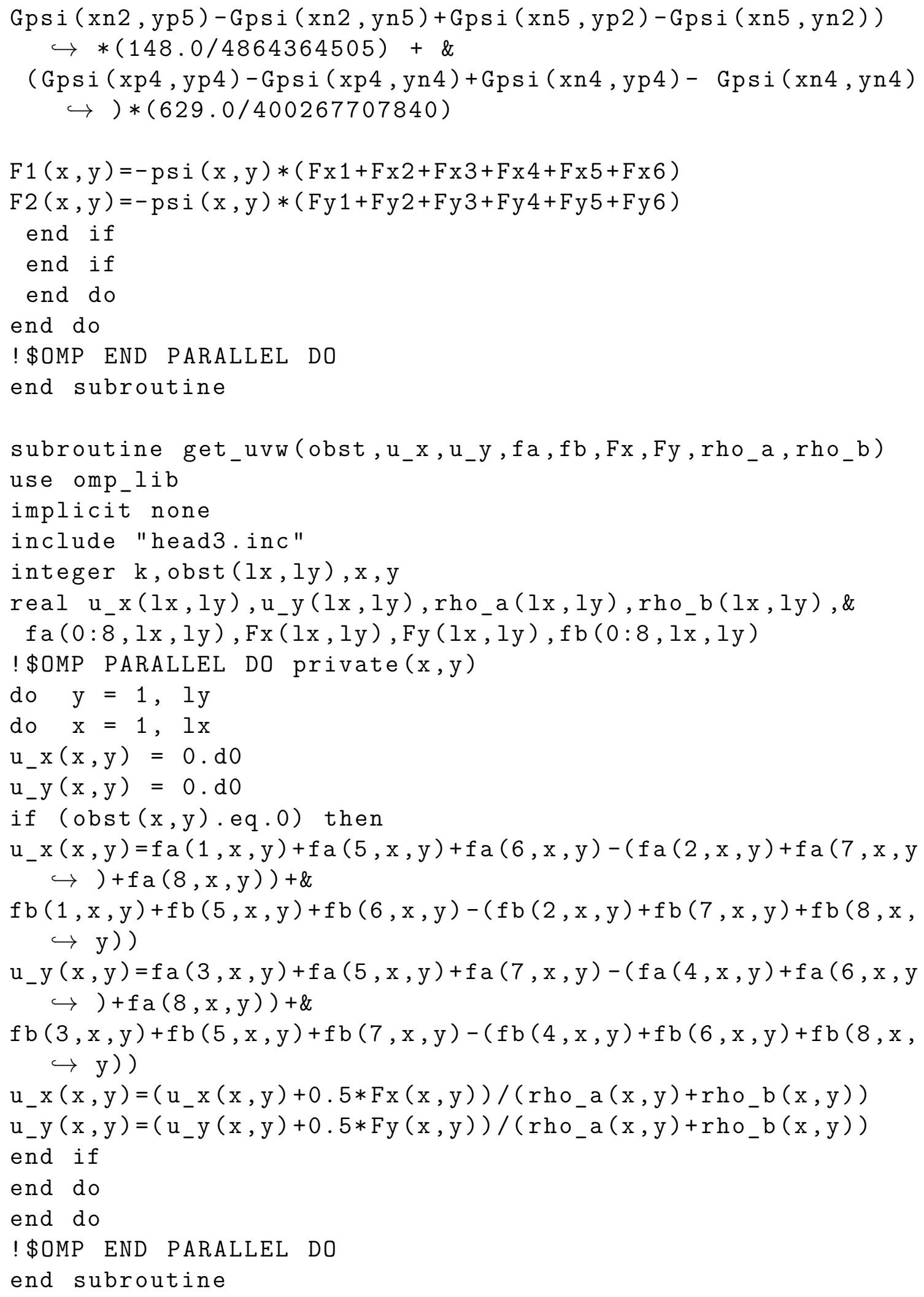




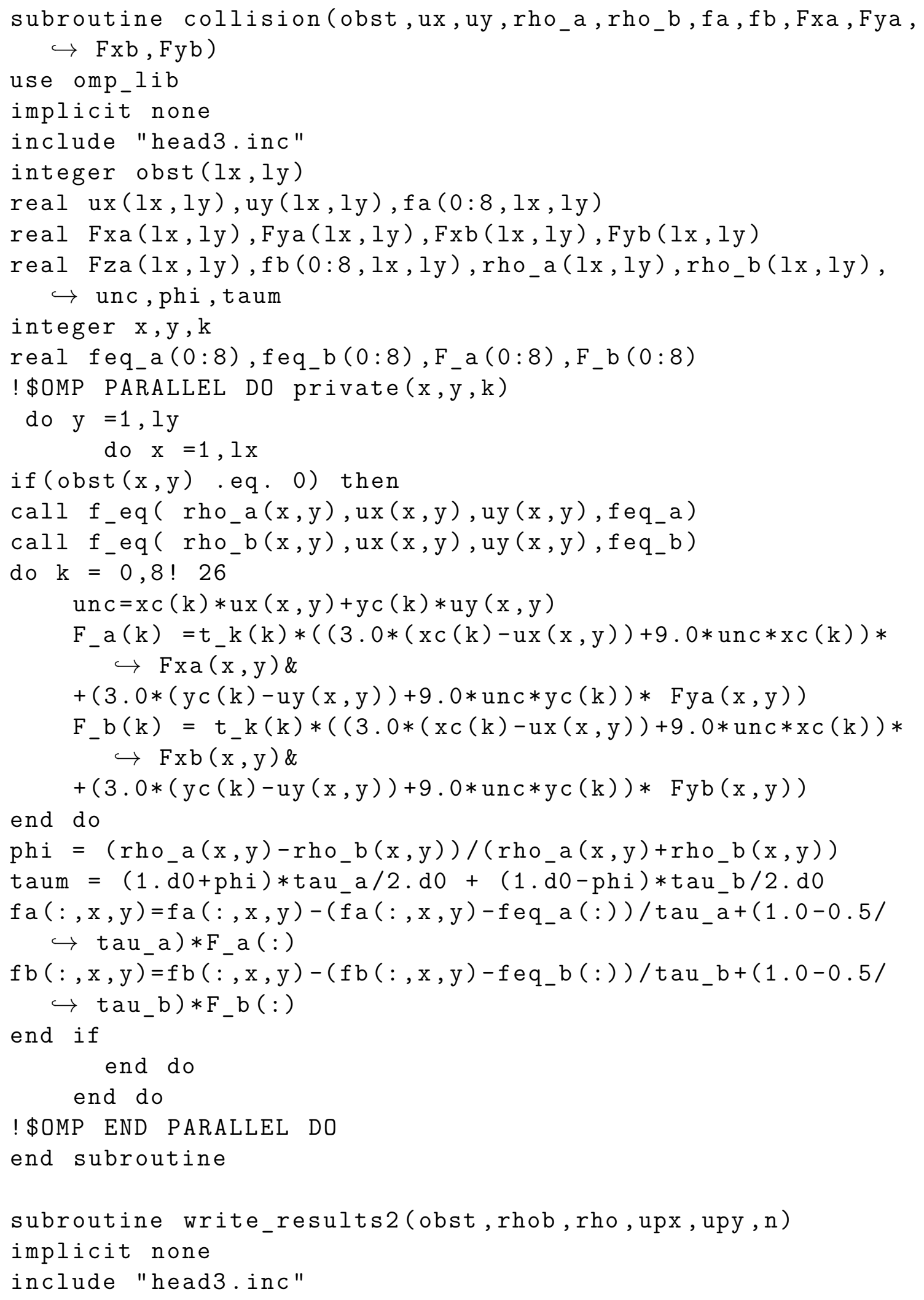




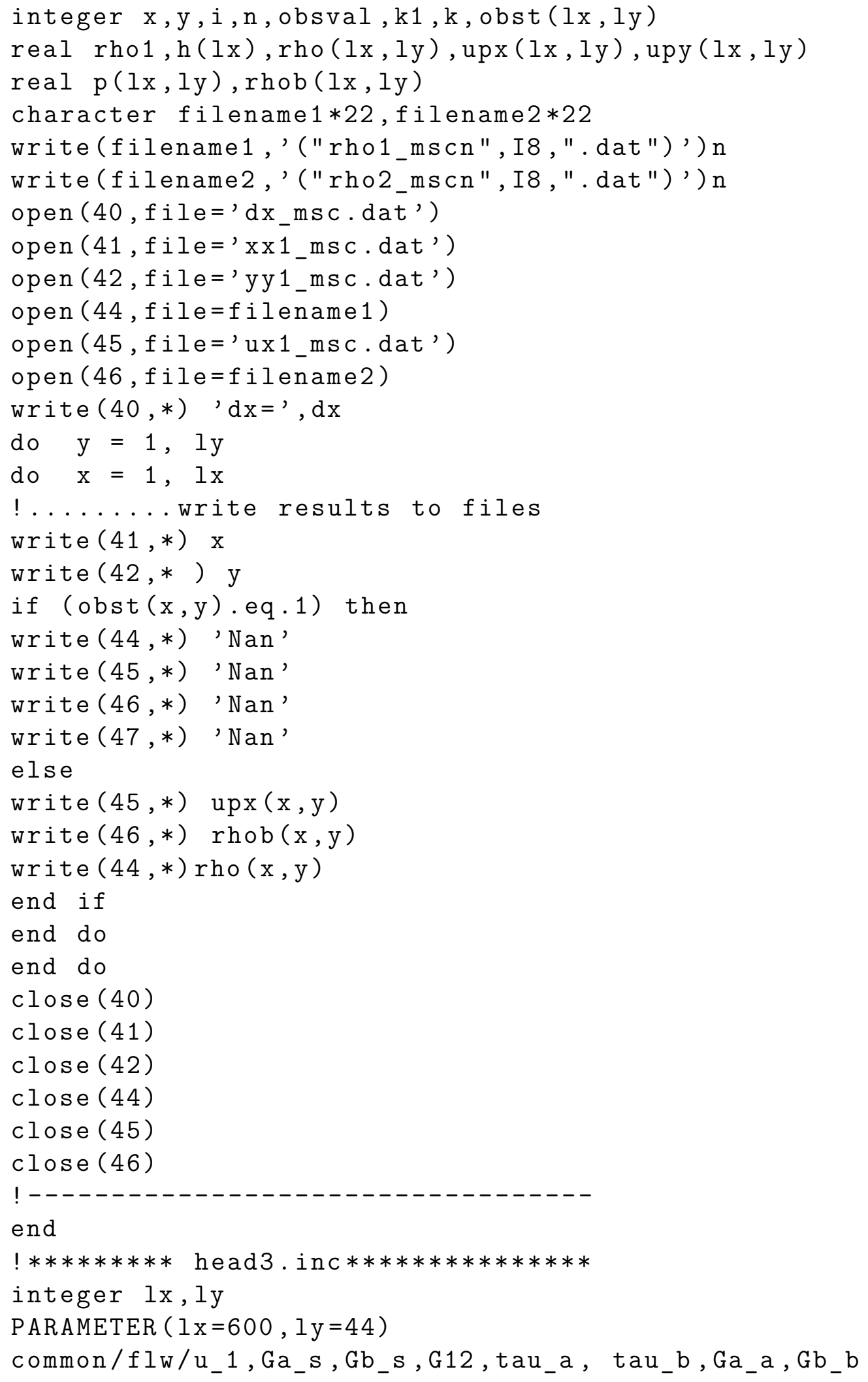




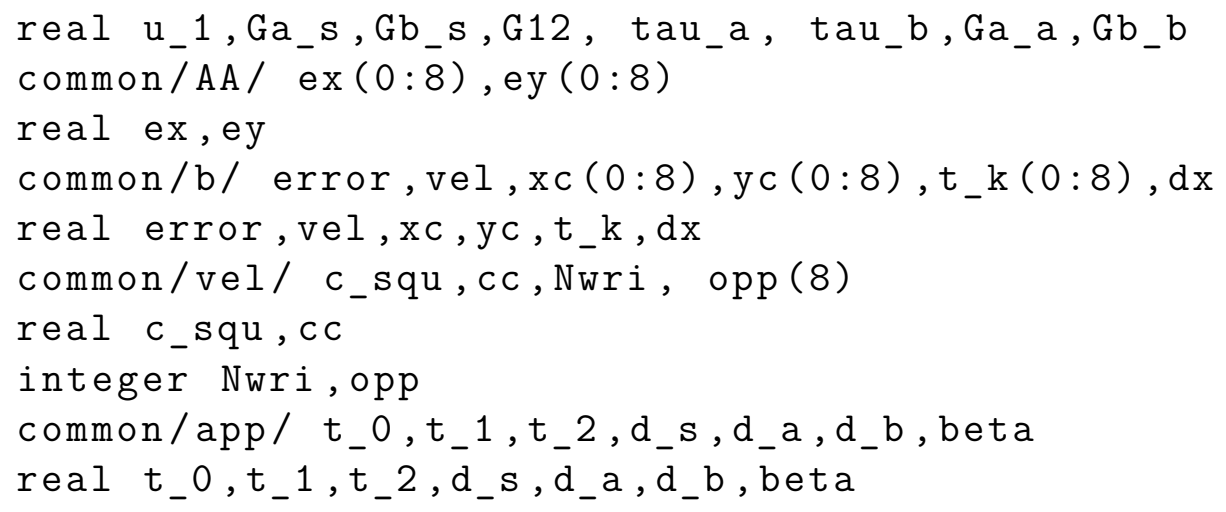




\section{A.4 Immiscible Case Flowchart}

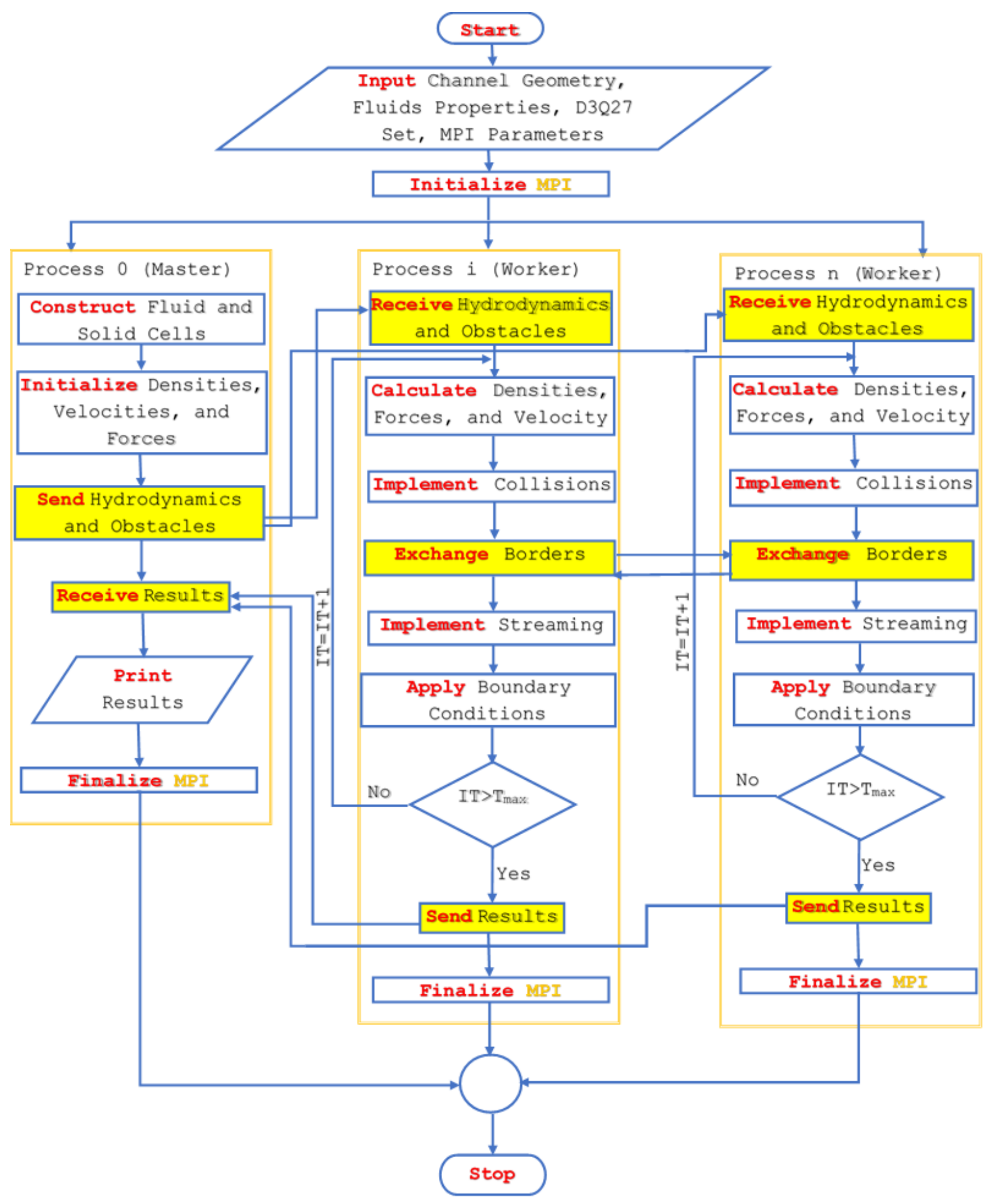

Figure A.4.1: Flowchart of the two-phase flow D3Q27 lattice Boltzmann method for a passive separation in a T-Junction. 


\section{A.5 Immiscible Case Code}

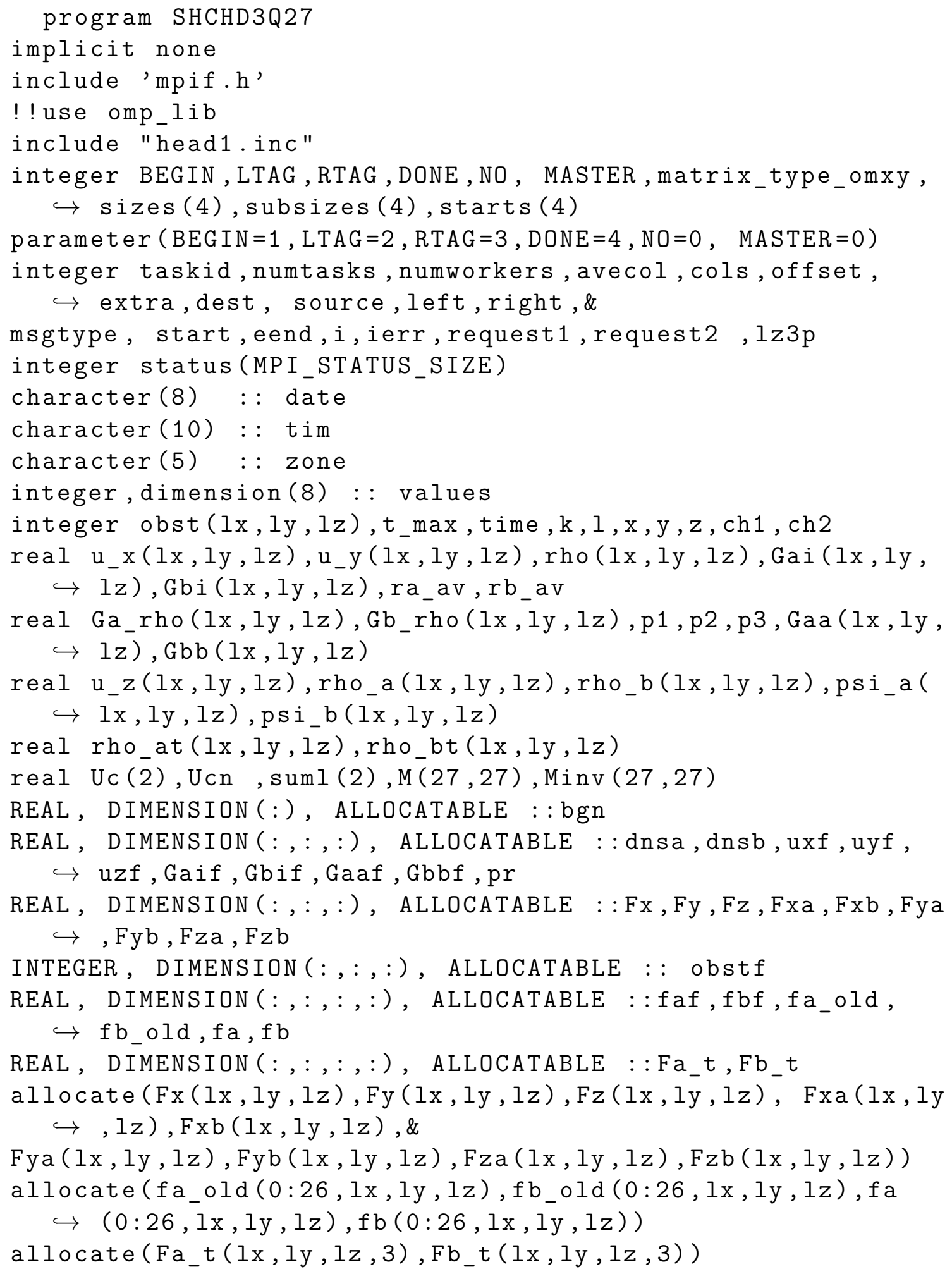


! Velocity vectors of the D3Q19 velocity model, $x c$, yc, zc $\hookrightarrow$ are

! the $x, y, z$ components of the vector, respectively. The $\hookrightarrow$ order of

! the velocity vector is slightly different from that in $\hookrightarrow$ Fig.1.1.

call date_and_time (date,tim, zone, values)

call date_and_time (DATE=date, $Z O N E=z o n e)$

call date_and_time (TIME=tim)

call date_and_time (VALUES=values)

print ' $(\mathrm{a}, 2 \mathrm{x}, \mathrm{a}, 2 \mathrm{x}, \mathrm{a})^{\prime}$, date, tim, zone

print ' $(8 \mathrm{i5})$ ', values

$\mathrm{xc}(0: 26)=(/ 0 . \mathrm{d} 0,1 . \mathrm{d} 0,-1 . \mathrm{d} 0,0 . \mathrm{d} 0,0 . \mathrm{d} 0,0 . \mathrm{d} 0,0 . \mathrm{d} 0,1$.

$\hookrightarrow \mathrm{d} 0,1 . \mathrm{d} 0,-1 . \mathrm{d} 0,-1 . \mathrm{d} 0,1 . \mathrm{d} 0,-1 . \mathrm{d} 0,1 . \mathrm{d} 0,-1 . \mathrm{d} 0,0$.

$\hookrightarrow \mathrm{d} 0,0 . \mathrm{d} 0,0 . \mathrm{d} 0,0 . \mathrm{d} 0, \&$

1.d $0,-1 . \mathrm{d} 0,1 . \mathrm{d} 0,-1 . \mathrm{d} 0,1 . \mathrm{d} 0,-1 . \mathrm{d} 0,1 . \mathrm{d} 0,-1 . \mathrm{d} 0 /)$

$\mathrm{yc}(0: 26)=(/ 0 . \mathrm{d} 0,0 . \mathrm{d} 0,0 . \mathrm{d} 0,1 . \mathrm{d} 0,-1.0 \mathrm{~d} 0,0 . \mathrm{d} 0,0 . \mathrm{d} 0,1$.

$\hookrightarrow \mathrm{d} 0,-1 . \mathrm{d} 0,1 . \mathrm{d} 0,-1 . \mathrm{d} 0,0 . \mathrm{d} 0,0 . \mathrm{d} 0,0 . \mathrm{d} 0,0 . \mathrm{d} 0,1 . \mathrm{d} 0$,

$\hookrightarrow 1 . \mathrm{d} 0,-1 \cdot \mathrm{d} 0,-1 . \mathrm{d} 0, \&$

$1 \cdot \mathrm{d} 0,-1 \cdot \mathrm{d} 0,1 \cdot \mathrm{d} 0,-1 \cdot \mathrm{d} 0,-1 \cdot \mathrm{d} 0,1 \cdot \mathrm{d} 0,-1 \cdot \mathrm{d} 0,1 \cdot \mathrm{d} 0 /)$

$z c(0: 26)=(/ 0 . d 0,0 . d 0,0 . d 0,0 . d 0,0 . d 0,1 . d 0,-1 . d 0,0 . d 0$

$\hookrightarrow, 0 . \mathrm{d} 0,0 . \mathrm{d} 0,0 . \mathrm{d} 0,1 . \mathrm{d} 0,1 . \mathrm{d} 0,-1 . \mathrm{d} 0,-1 . \mathrm{d} 0,1 . \mathrm{d} 0$,

$\hookrightarrow-1 . \mathrm{d} 0,1 . \mathrm{d} 0,-1 . \mathrm{d} 0, \&$

1.d $0,-1 \cdot d 0,-1 \cdot d 0,1 \cdot d 0,-1 \cdot d 0,1 \cdot d 0,1 \cdot d 0,-1 \cdot d 0 /)$

$\operatorname{opp}(1: 26)=(/ 2,1,4,3,6,5,10,9,8,7,14,13,12$,

$\hookrightarrow 11,18,17,16,15,20,19,22,21,24,23,26,25 /)$

! Lattice speed

$\mathrm{cc}=1 \cdot \mathrm{d} 0$

! Square of sound speed in the LBM

$c_{-}$squ $=c c * c c / 3 . d 0$

! Lattice Weights

$t_{-} 0=8 \cdot \mathrm{d} 0 / 27 \cdot \mathrm{d} 0$

$t_{-} 1=2 \cdot \mathrm{d} 0 / 27 \cdot \mathrm{d} 0$

$t_{-} 2=1 . \mathrm{d} 0 / 54 . \mathrm{d} 0$

$t_{-} 3=1 \cdot \mathrm{d} 0 / 216 \cdot \mathrm{d} 0$

$t_{-} k(0)=t_{-} 0$

do $1 \mathrm{k}=1,6$

$t_{-} k(k)=t_{-} 1$

1 continue

do $2 \mathrm{k}=7,18$

$t_{-} k(k)=t_{-} 2$

2 continue 


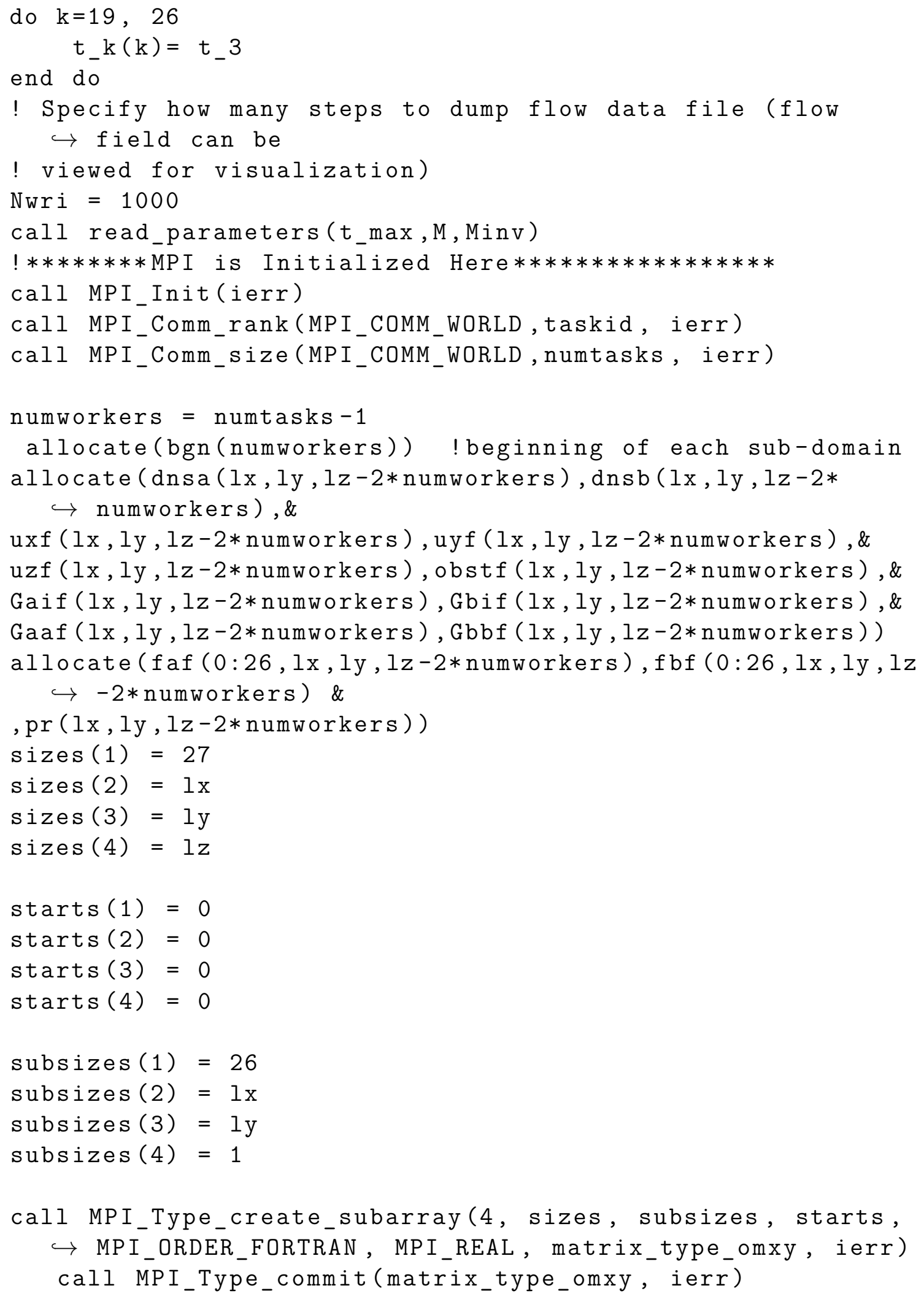




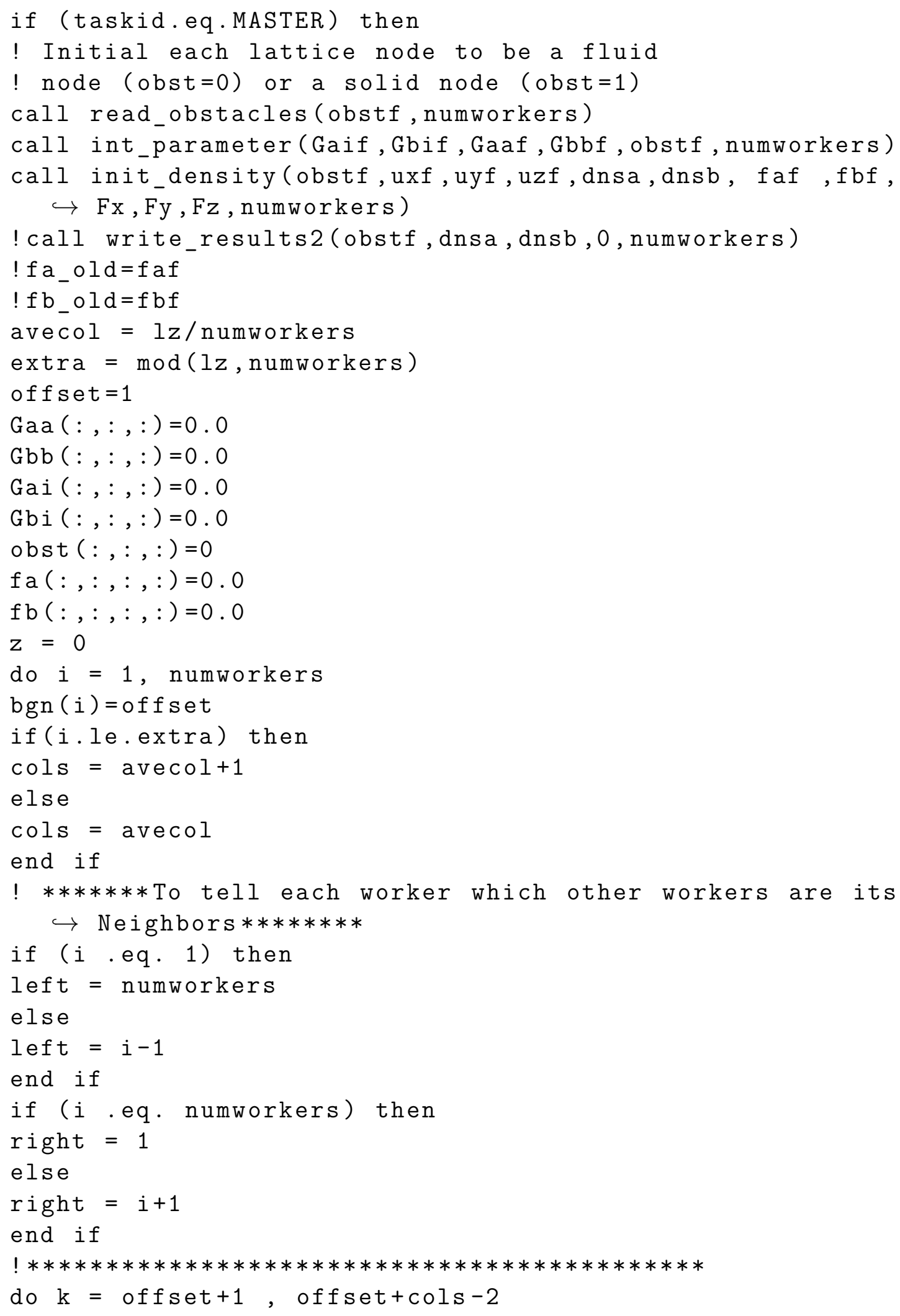




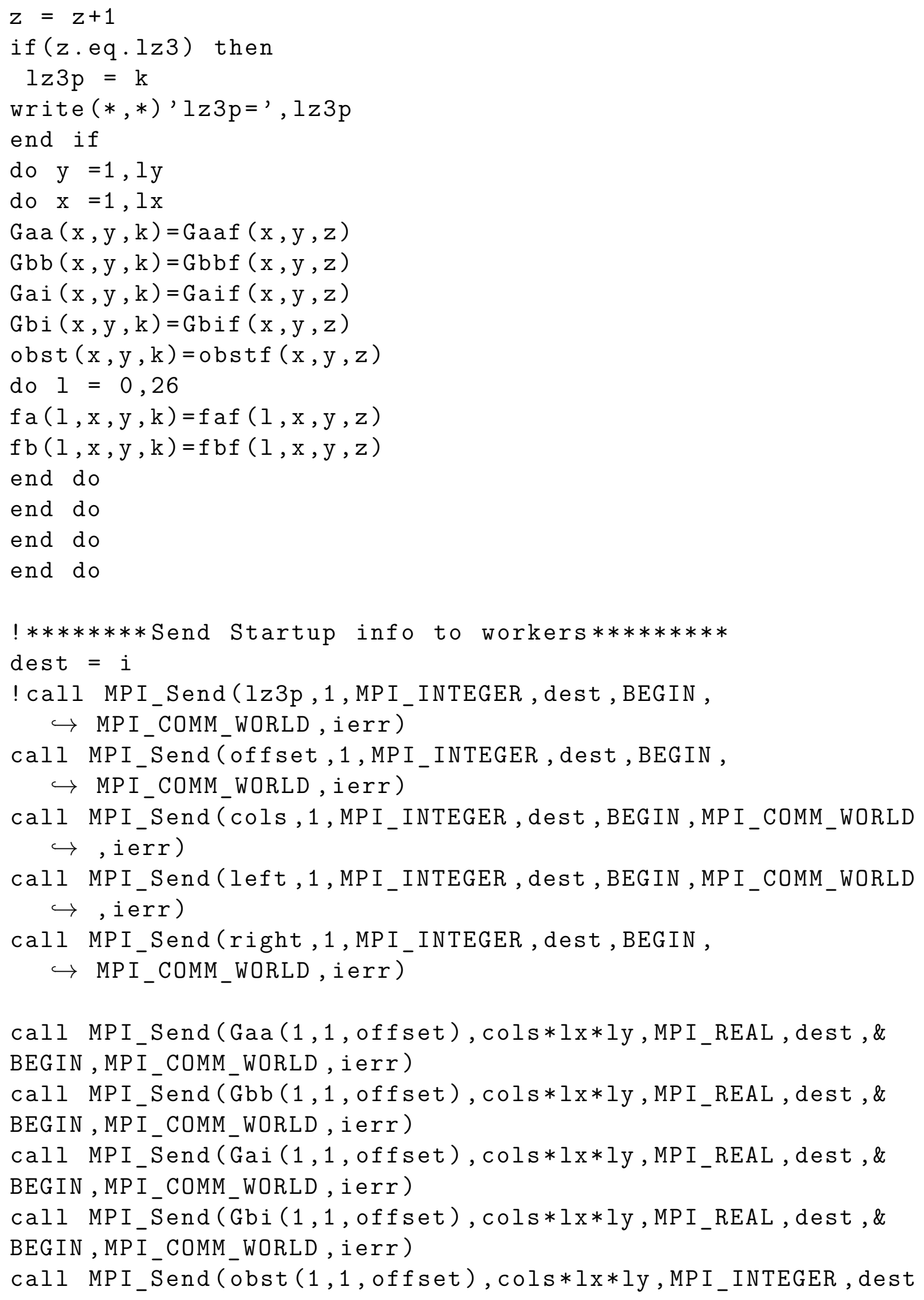




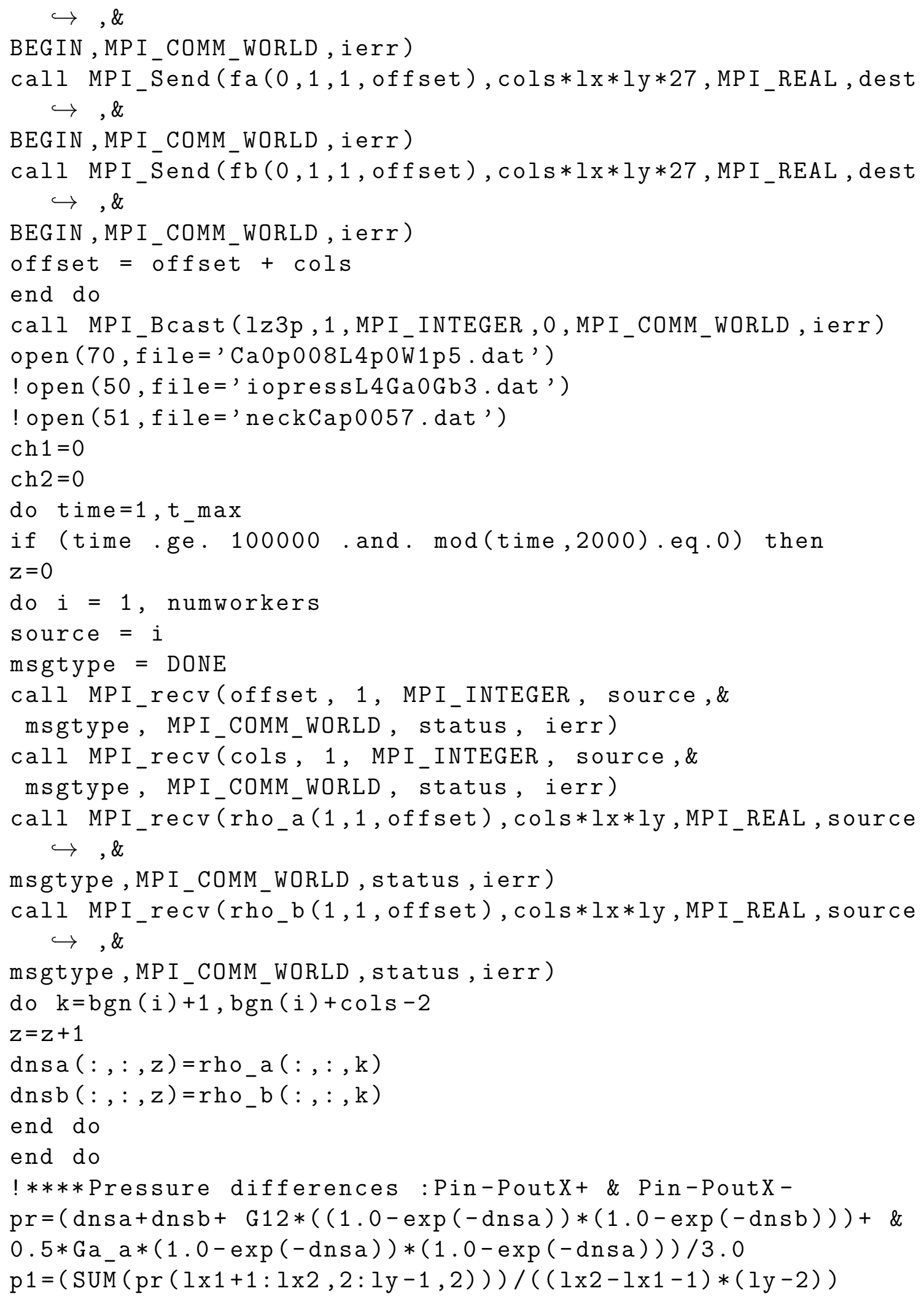




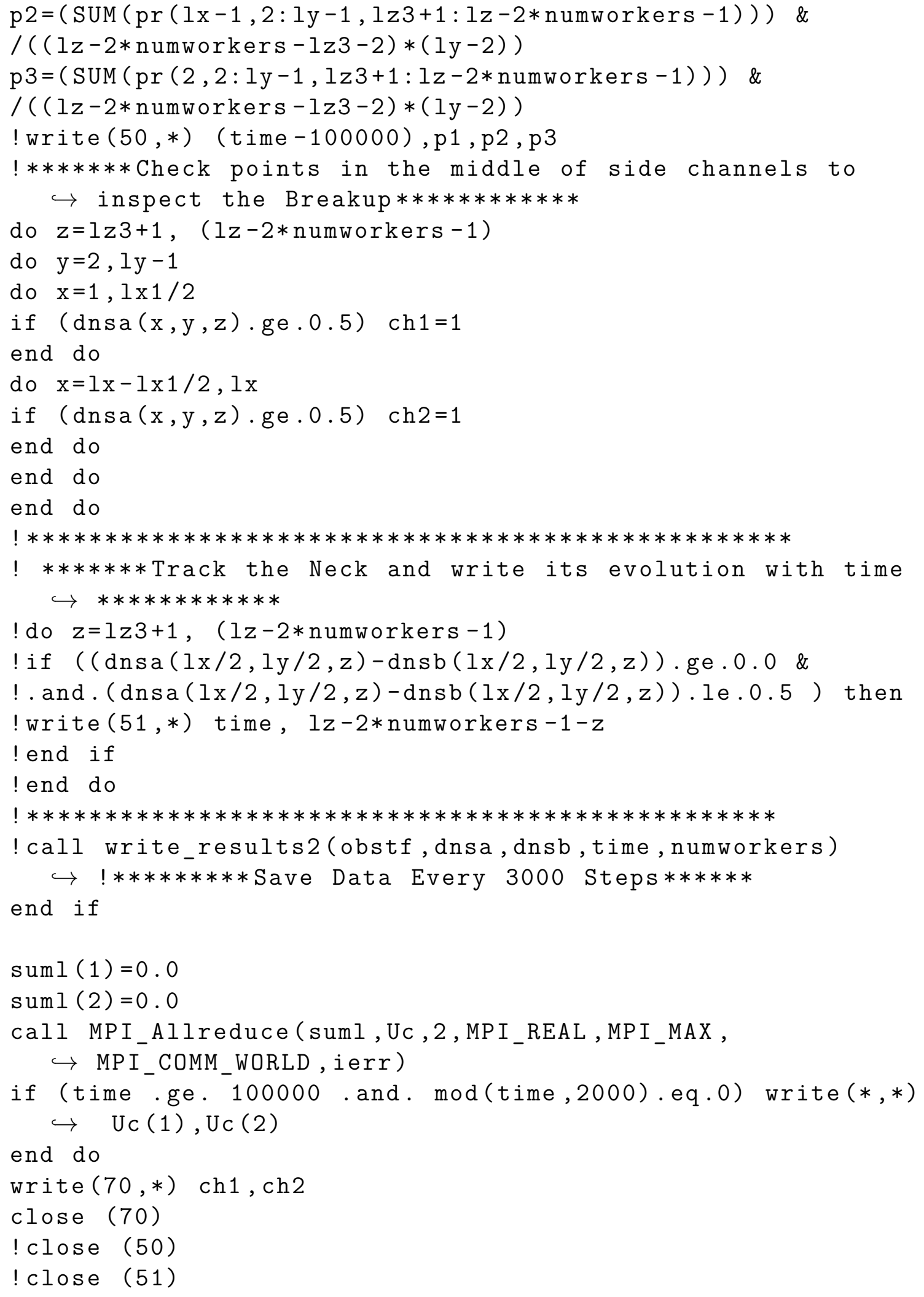




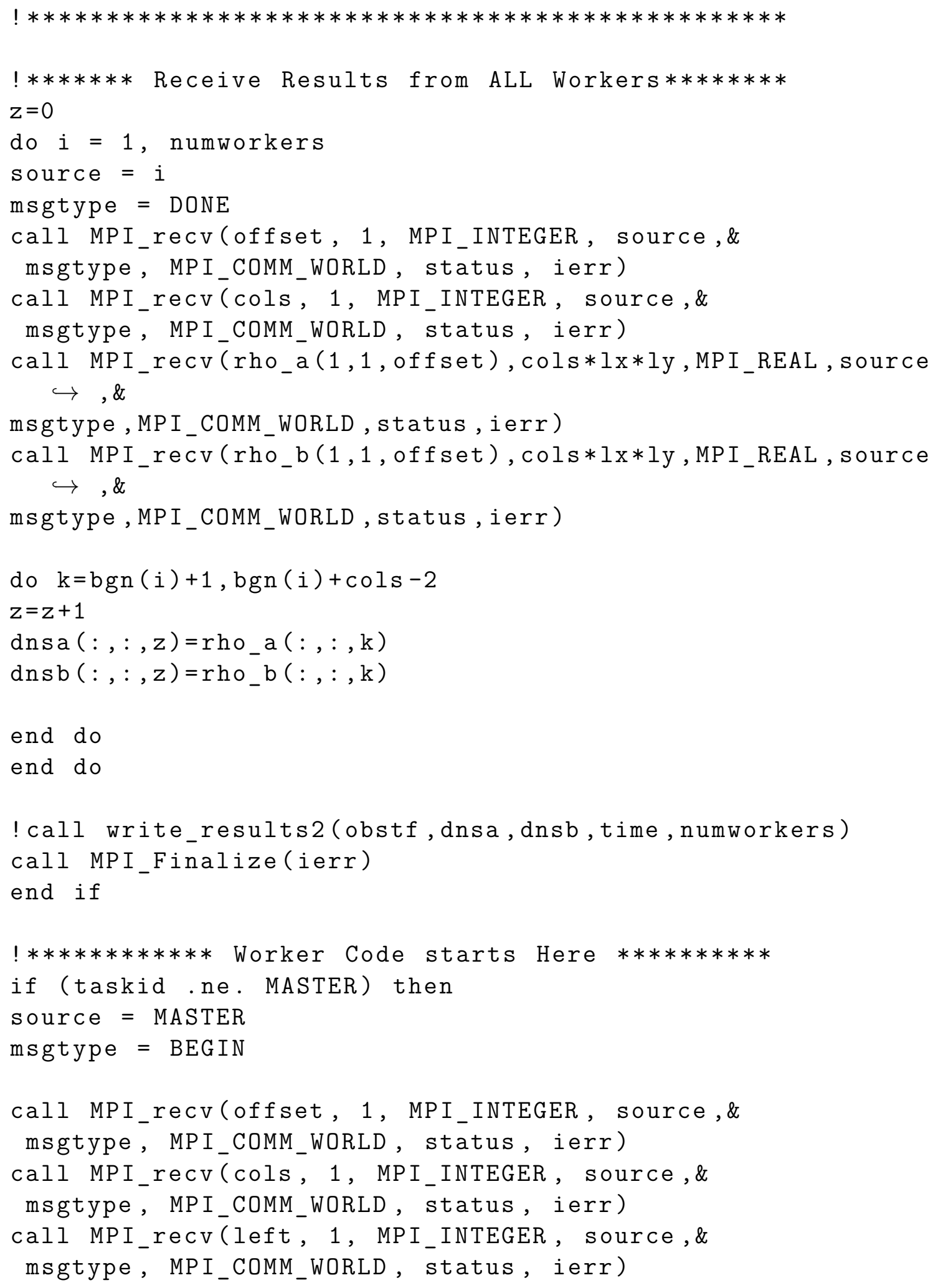


call MPI_recv(right, 1, MPI_INTEGER, source,\&

msgtype, MPI_COMM_WORLD, status, ierr)

call MPI_recv (Gaa $\left(\overline{1}, 1\right.$, offset), cols $* 1 x * 1 y, M P I \_R E A L$, source, \& BEGIN, MPI_COMM_WORLD, status, ierr)

call MPI_recv (Gbb(1,1, offset), cols*lx*ly, MPI_REAL, source,\& BEGIN, MPI_COMM_WORLD, status, ierr)

call MPI_recv (Gai (1,1, offset), cols*lx*ly, MPI_REAL, source, \& BEGIN, MPI_COMM_WORLD, status, ierr)

call MPI_recv (Gbi $\left(1,1\right.$, offset), cols $* 1 x * 1 y, M P I \_R E A L$, source, \& BEGIN, MPI_COMM_WORLD, status, ierr)

call MPI_recv(obst $(1,1$, offset), cols*lx*ly, MPI_INTEGER,

$\hookrightarrow$ source, \&

BEGIN, MPI_COMM_WORLD, status, ierr)

call MPI_recv(fa( $0,1,1$, offset $), \operatorname{cols} * l x * 1 \mathrm{y} * 27$, MPI_REAL,

$\hookrightarrow$ source, \&

msgtype,MPI_COMM_WORLD, status, ierr)

call MPI_recv(fb(0,1,1, offset), cols*lx*ly*27, MPI_REAL,

$\hookrightarrow$ source, \&

msgtype,MPI_COMM_WORLD, status, ierr)

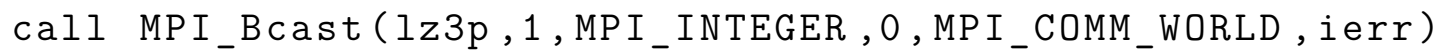

! $* * * * * * * *$ Find the borders of each task $* * * * * * * * * * * * * * * * *$

start $=$ offset +1

eend $=$ offset $+\operatorname{cols}-2$

do time $=1, t_{-} \max$

if (time .ge. 100000 .and. $\bmod (t i m e, 2000)$.eq.0) then

msgtype $=$ DONE

call MPI_Send (offset, 1, MPI_INTEGER, MASTER, \&

msgtype, MPI_COMM_WORLD, ierr)

call MPI_Send(cols, 1, MPI_INTEGER, MASTER, \&

msgtype, MPI_COMM_WORLD, ierr)

call MPI_Send (rho_a $(1,1$, offset), cols*lx*ly, MPI_REAL, MASTER $\hookrightarrow \quad, \&$

msgtype,MPI_COMM_WORLD, ierr)

call MPI_Send (rho_b(1,1, of fset), cols*lx*ly, MPI_REAL, MASTER $\hookrightarrow \quad, \&$

msgtype,MPI_COMM_WORLD, ierr)

if (taskid.eq.1) write(*,*) time !, ' lz3p=', lz3p

end if 


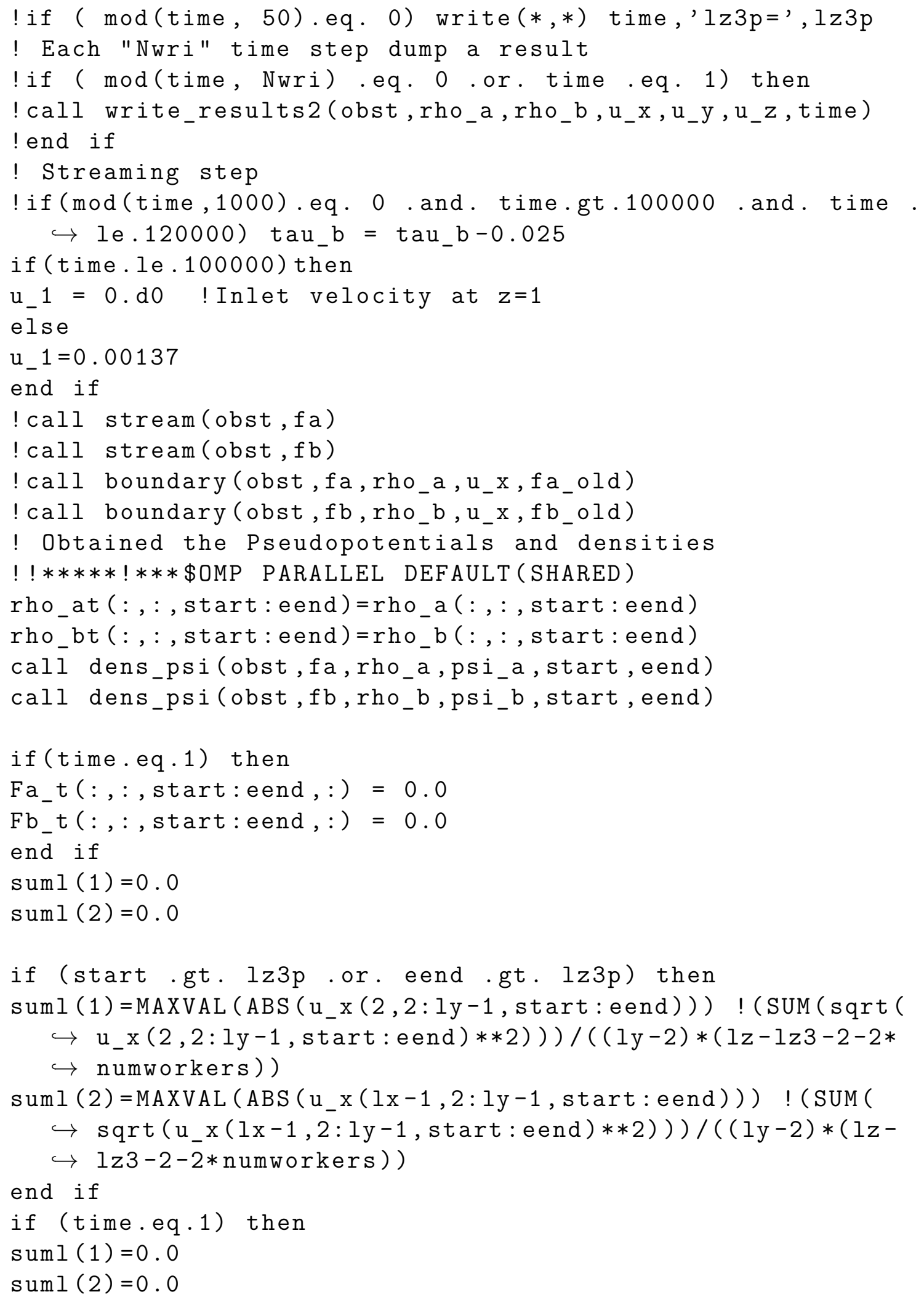


end if

call MPI_Allreduce (suml, Uc, 2, MPI_REAL, MPI_MAX,

$\hookrightarrow$ MPI_COMM_WORLD, ierr)

! ******Force at the inlet and outlets by Advective

$\hookrightarrow$ Boundary Conditions $* * * * * *$

call int_force(obst,psi_a,psi_b, Gai, Gbi, Gaa, Gbb, Fx, Fy, Fz,

$\hookrightarrow$ Fxa,Fxb,Fya,Fyb \&

, Fza, Fzb, u_x, time, Fa_t, Fb_t, start, eend, left, right, Uc

$\hookrightarrow$ (1), Uc (2), 1z3p)

call get_uvw(obst, u_x, u_y, u_z, fa, fb, Fx, Fy, Fz, rho_a, rho_b ,

$\hookrightarrow$ start, eend)

call old_pop(fa,fa_old, start, eend)

call old_pop (fb, fb_old, start, eend)

call collision(obst, $u_{-} x, u_{-} y, u_{-} z$, rho_a, rho_b, fa, fb, Fxa, Fya , $\hookrightarrow \mathrm{Fza}, \&$

Fxb, Fyb, Fzb, start, eend, M, Minv)

call MPI_Sendrecv(fa(1,1,1, start),1, matrix_type_omxy, left $\hookrightarrow$, , \&

RTAG, fa $(1,1,1$, eend +1), 1, matrix_type_omxy, right , \&

RTAG, MPI_COMM_WORLD, status, ierr)

! call MPI_Wait(request1, status, Ierr)

call MPI_Sendrecv(fa( $1,1,1$, eend ), 1, matrix_type_omxy, right $\hookrightarrow, \&$

LTAG, fa $(1,1,1$, start-1), 1, matrix_type_omxy, left, \&

LTAG, MPI_COMM_WORLD, status, ierr)

call MPI_Sendrecv(fb(1,1,1, start),1, matrix_type_omxy, left $\hookrightarrow, \&$

RTAG, fb $(1,1,1$, eend +1), 1, matrix_type_omxy, right , \&

RTAG, MPI_COMM_WORLD, status, ierr)

! call MPI_Wait (request3, status, Ierr)

call MPI_Sendrecv(fb(1,1,1, eend), 1, matrix_type_omxy, right $\hookrightarrow$, , \&

LTAG,$f b(1,1,1$, start-1), 1 , matrix_type_omxy, left, \&

LTAG,MPI_COMM_WORLD, status, ierr)

! call MPI_Barrier(MPI_COMM_WORLD, ierr) 
call stream (obst, fa, start, eend, left, right)

call stream (obst, fb, start, eend, left, right)

rho_a $(1 \times 1+1: 1 \times 2,2: 1 y-1,2)=0.0$

call boundary (obst, fa, rho_a, rho_at, $u_{-} x, u_{-} y, u_{-} z, f_{-} o l d, t i m e$ $\hookrightarrow$, start, eend, Uc (1), Uc (2), lz3p)

call boundary (obst, fb, rho_b, rho_bt, u_x, u_y, u_z, fb_old, time $\hookrightarrow$, start, eend, Uc (1), Uc (2), lz3p)

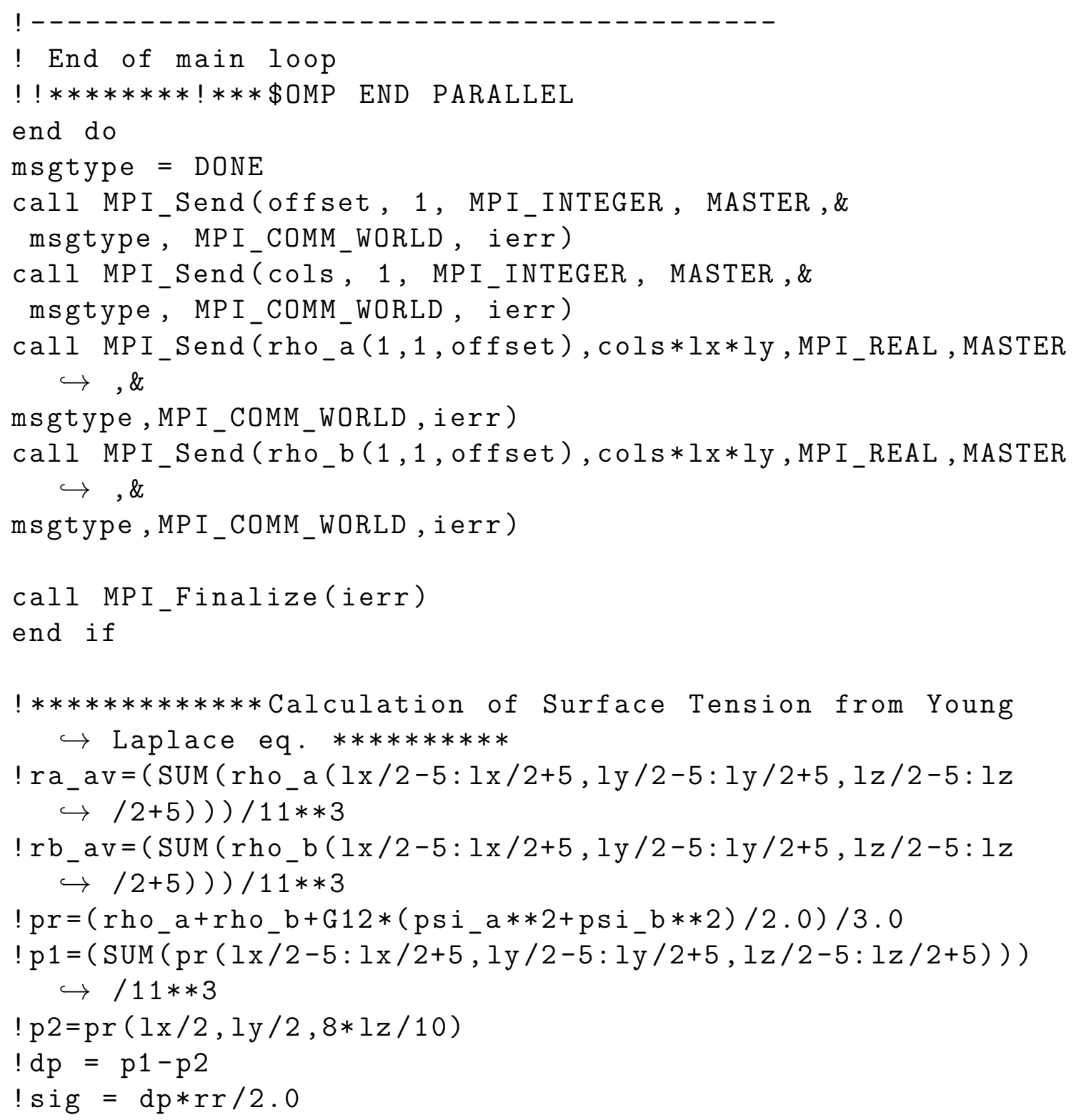




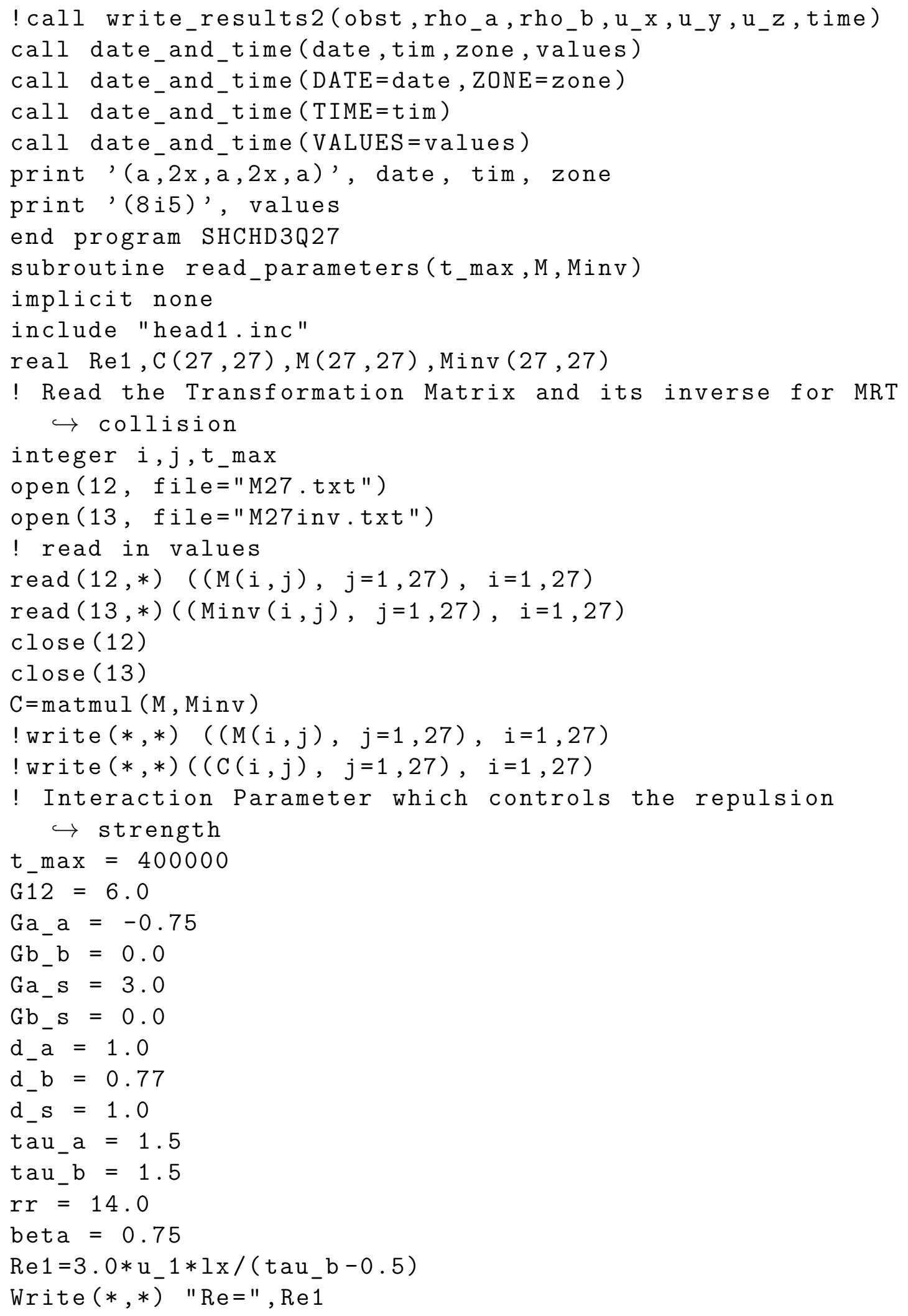




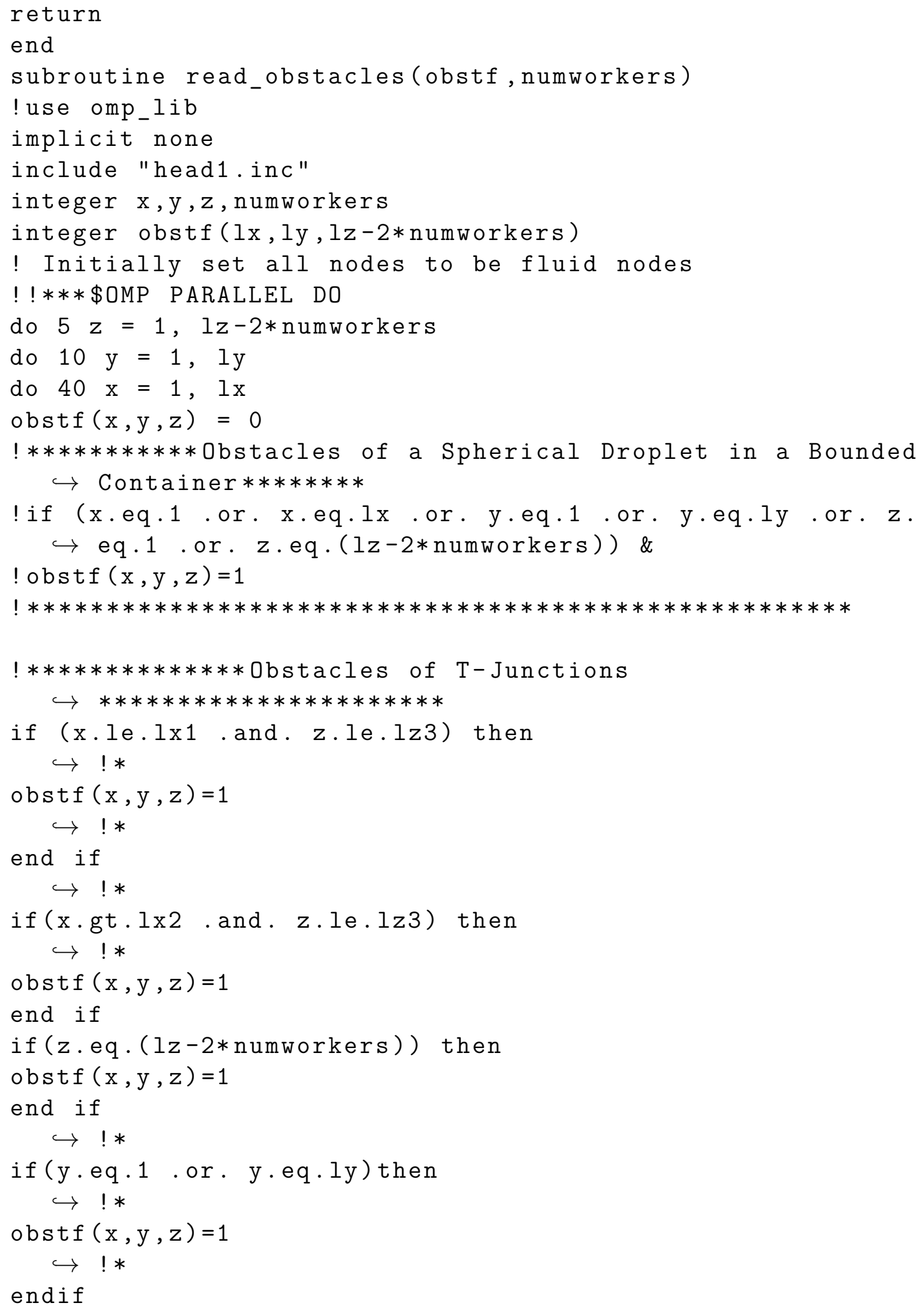




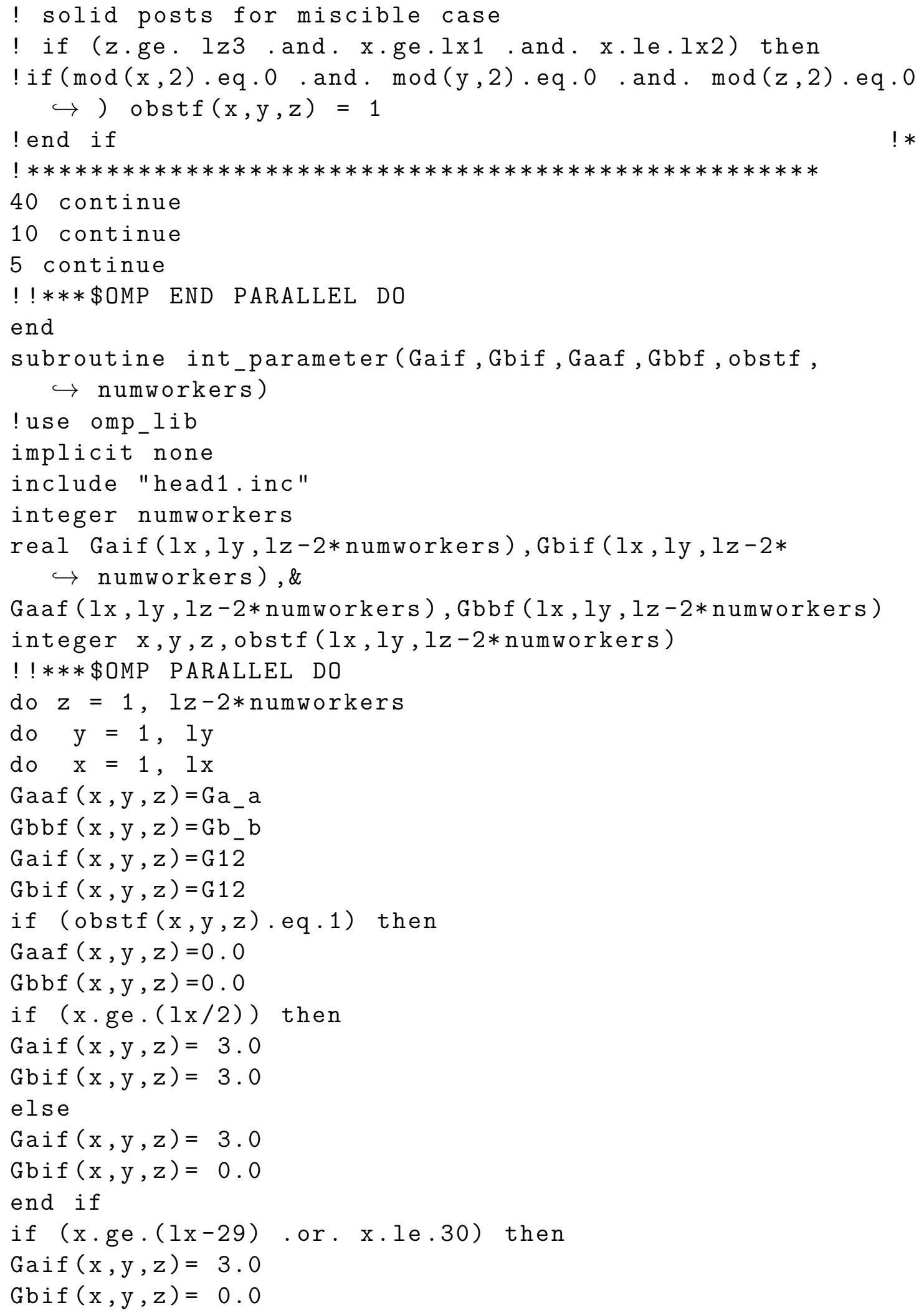




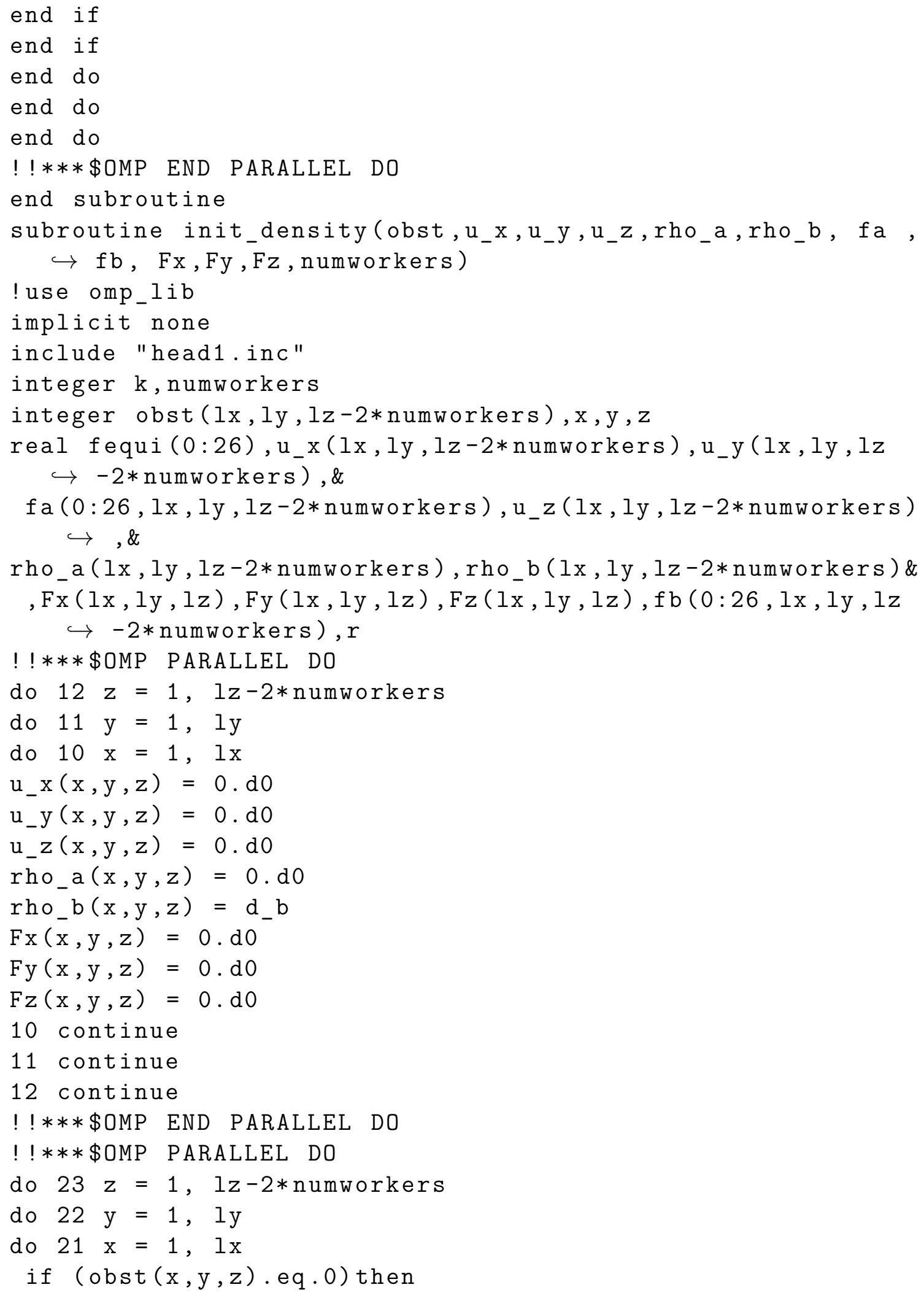




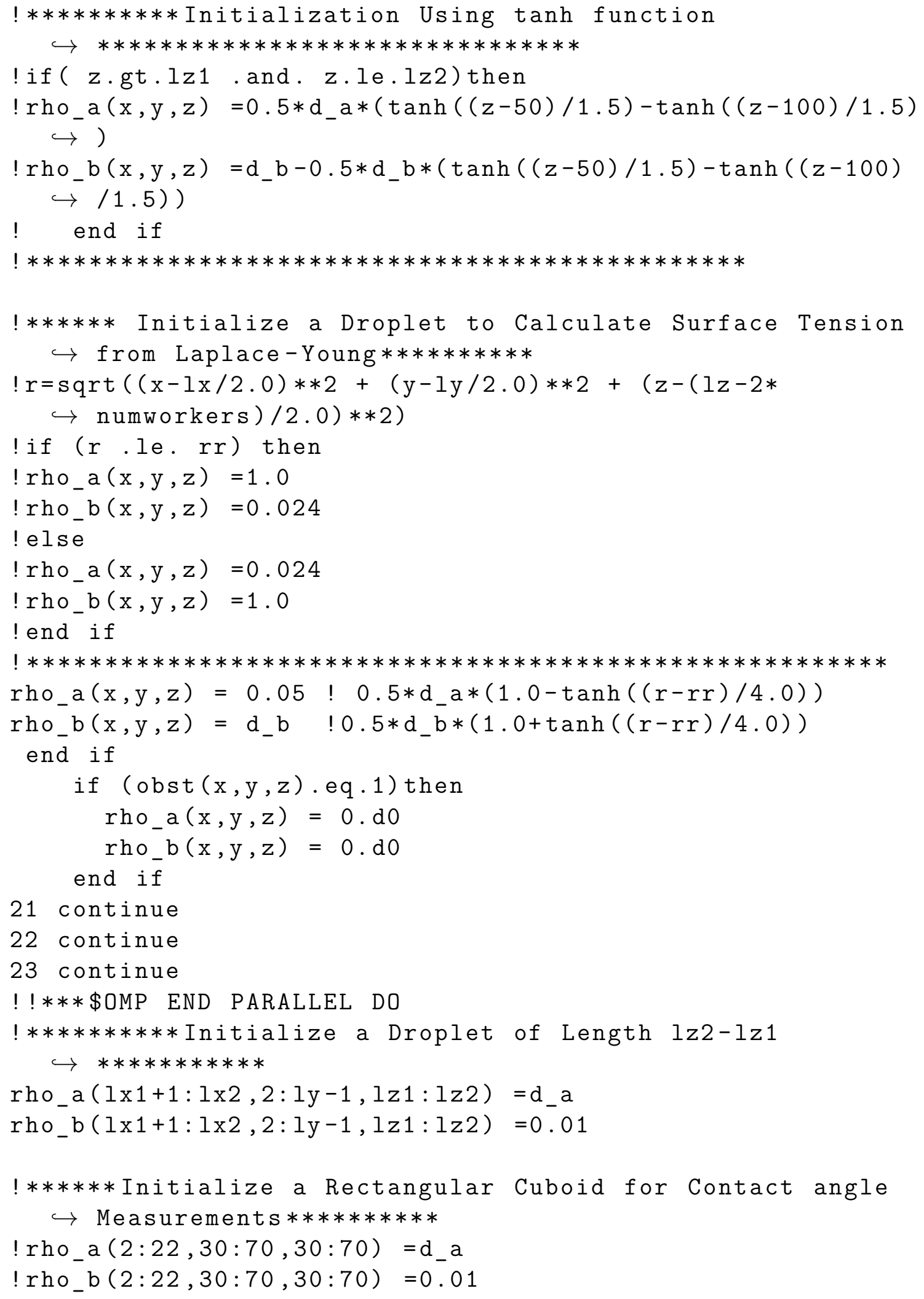




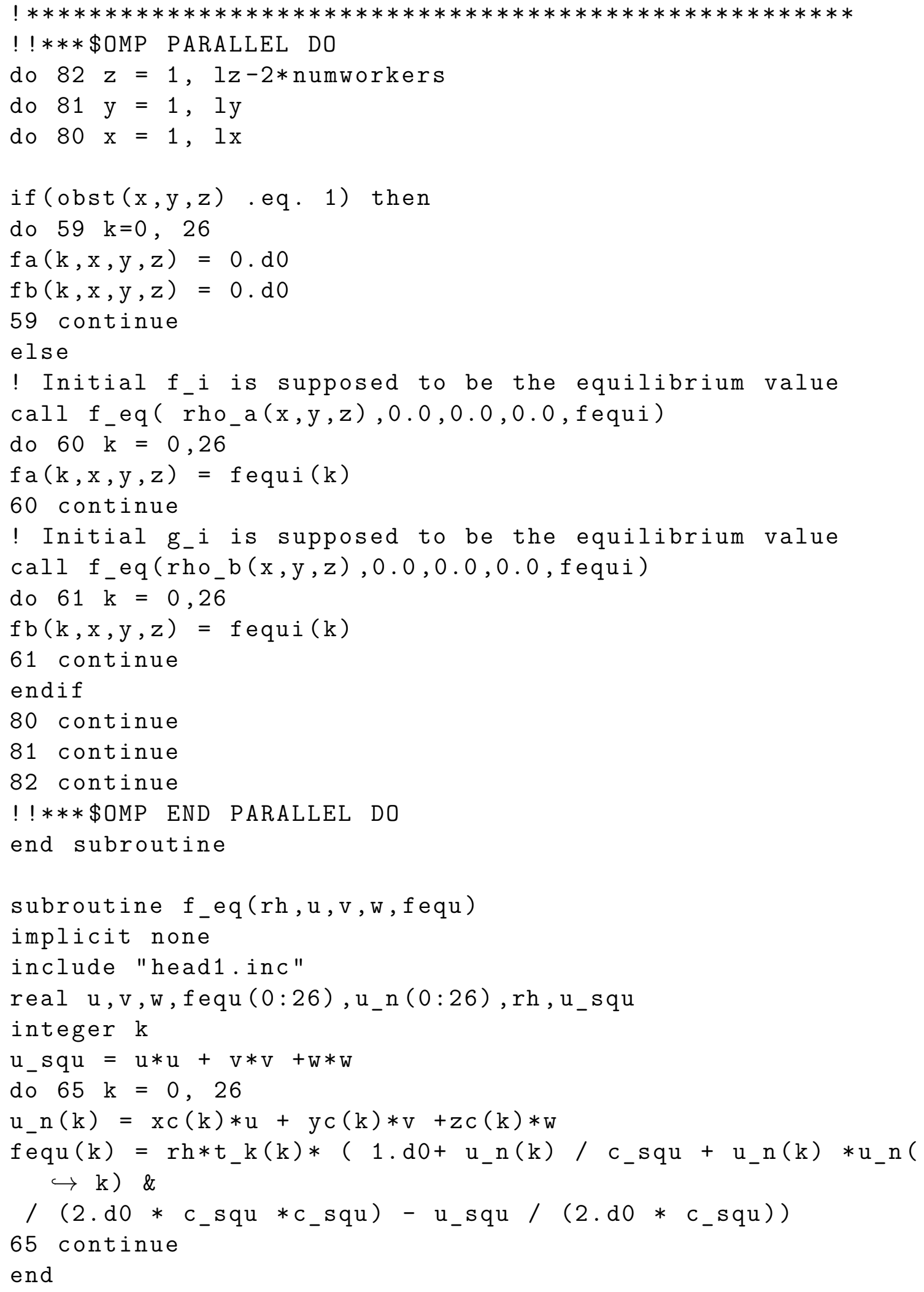




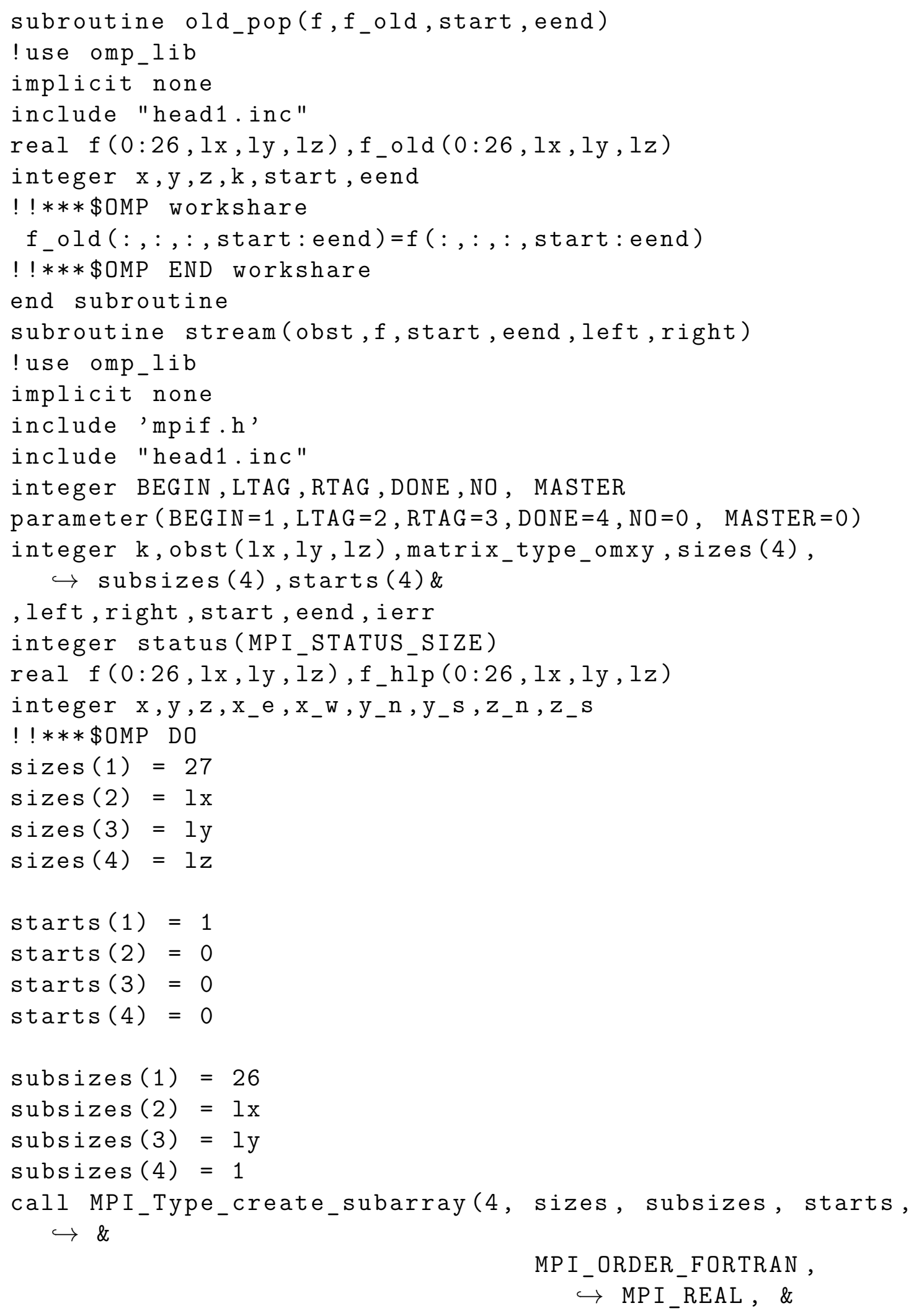




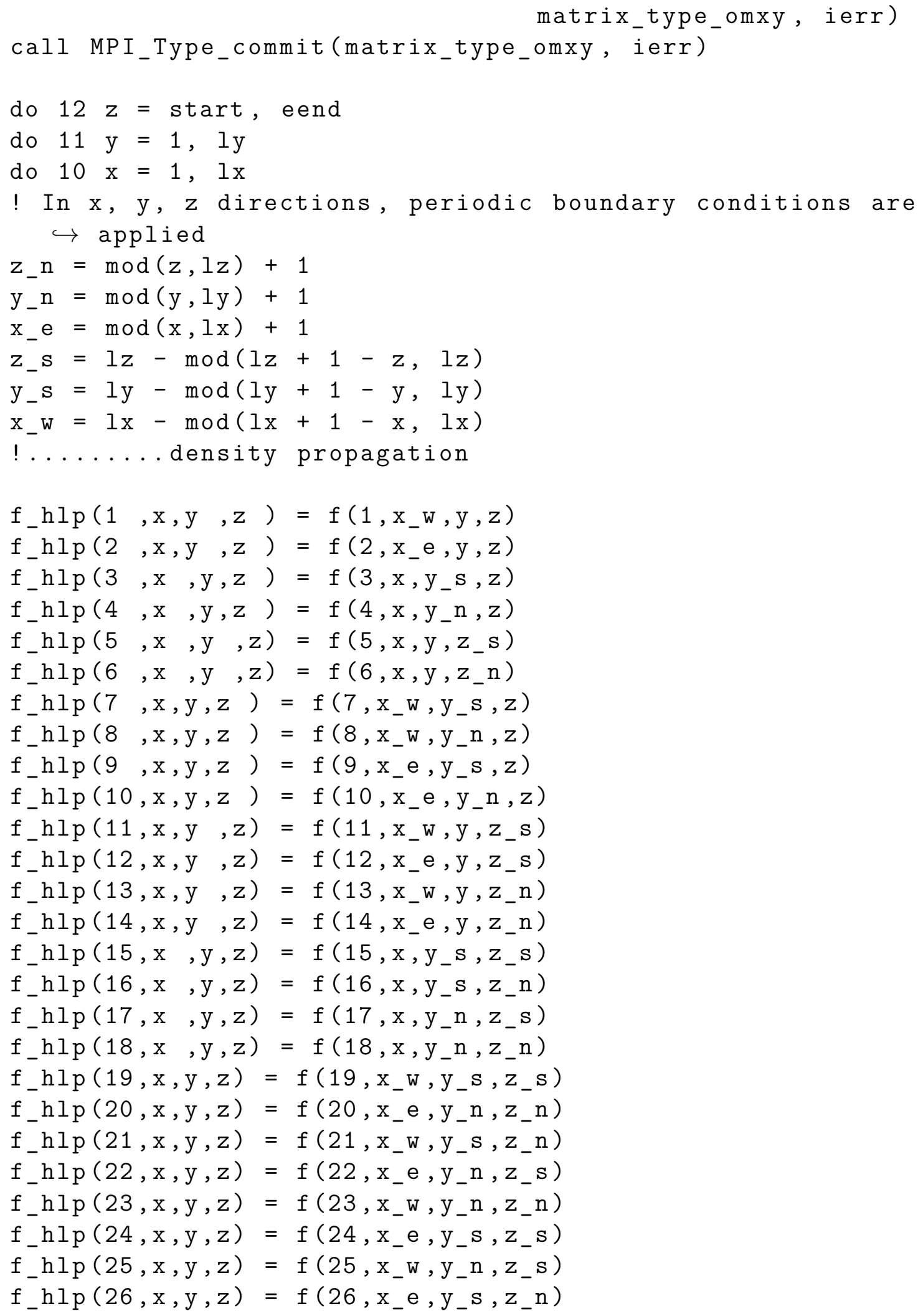




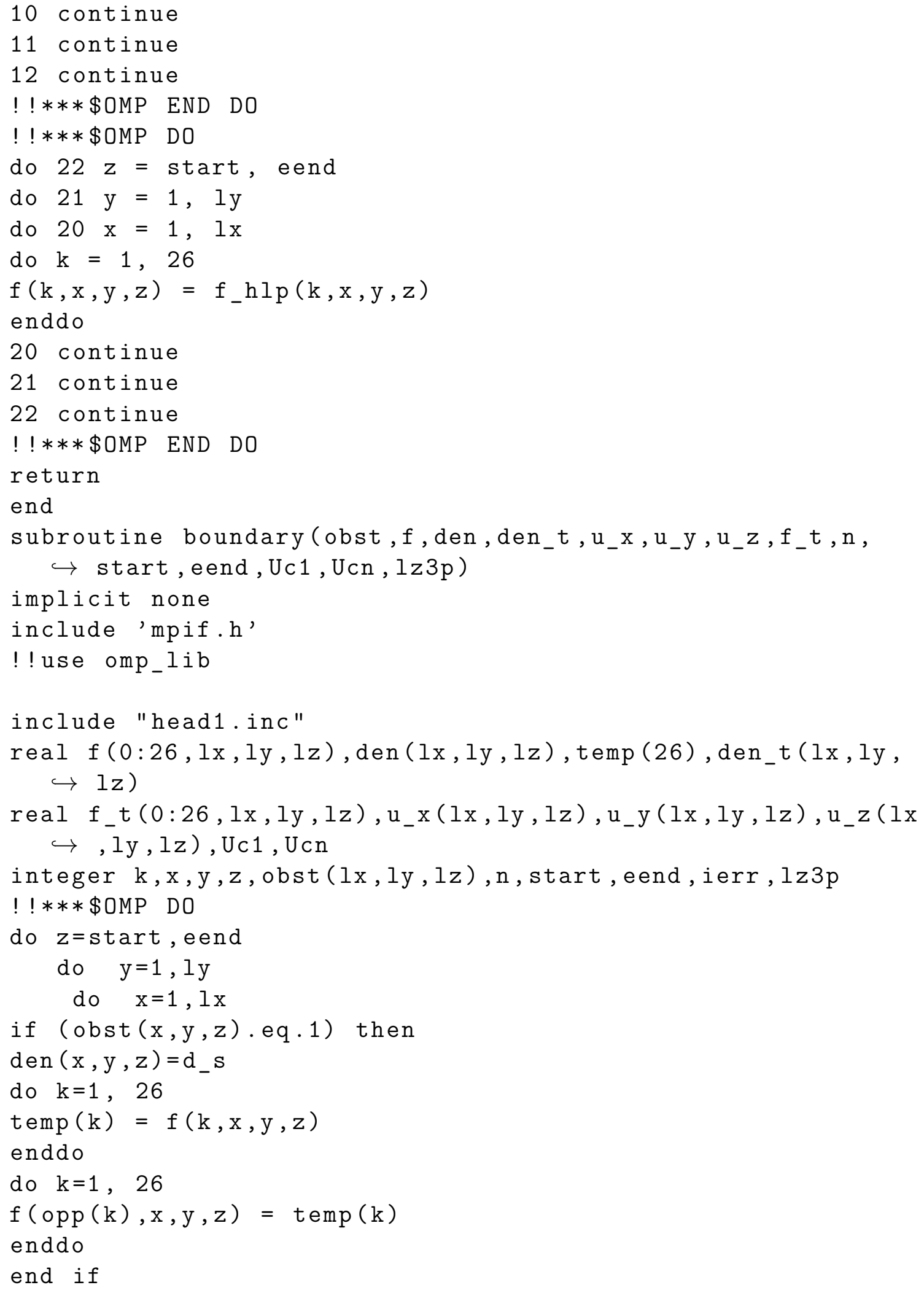


enddo

enddo

enddo

$! ! * * * \$ O M P$ END DO

! Apply the Half-way Bounce Back on the first inlet with u $\hookrightarrow=\mathrm{u} 1$

! this is used to update $\mathrm{f} 5 \mathrm{f} 11 \mathrm{f} 12 \mathrm{f} 15 \mathrm{f} 17$ at $\mathrm{Z}=1$

$! ! * * * \$ O M P$ DO

!************Inlet Boundary Conditions Using Velocity

$\hookrightarrow$ Adjudted Bounce Back********

if (start.eq. 2) then

do $y=2,1 y-1$

do $\mathrm{x}=1 \mathrm{x} 1+1,1 \mathrm{x} 2$

$f(5, x, y$, start $)=f(o p p(5), x, y$, start $)-6 . d 0 * t_{-} k(o p p(5)) * \operatorname{den}(x$, $\hookrightarrow \mathrm{y}$, start $) * \mathrm{u}_{-} 1 * \mathrm{zc}(\mathrm{opp}(5))$

$f(11, x, y$, start $)=f(o p p(11), x, y$, start $)-6 . d 0 * t \_k(o p p(11)) *$ den

$\hookrightarrow(\mathrm{x}, \mathrm{y}, \mathrm{start}) * \mathrm{u}_{-} 1 * \mathrm{zc}(\mathrm{opp}(11))$

$f(12, x, y$, start $)=f(o p p(12), x, y$, start $)-6 . d 0 * t \_k(o p p(12)) *$ den

$\hookrightarrow(\mathrm{x}, \mathrm{y}, \mathrm{start}) * \mathrm{u}_{-} 1 * \mathrm{zc}(\mathrm{opp}(12))$

$f(15, x, y$, start $)=f(o p p(15), x, y$, start $)-6 . d 0 * t \_k(o p p(15)) *$ den

$\hookrightarrow \quad(\mathrm{x}, \mathrm{y}, \mathrm{start}) * \mathrm{u}_{-} 1 * \mathrm{zc}(\mathrm{opp}(15))$

$f(17, x, y$, start $)=f(o p p(17), x, y$, start $)-6 . d 0 * t \_k(o p p(17)) *$ den

$\hookrightarrow(\mathrm{x}, \mathrm{y}, \mathrm{start}) * \mathrm{u}_{-} 1 * \mathrm{zc}(\mathrm{opp}(17))$

$f(19, x, y$, start $)=f(o p p(19), x, y$, start $)-6 . d 0 * t \_k(o p p(19)) *$ den

$\hookrightarrow(\mathrm{x}, \mathrm{y}, \mathrm{start}) * \mathrm{u}_{-} 1 * \mathrm{zc}(\mathrm{opp}(19))$

$f(22, x, y$, start $)=f(o p p(22), x, y$, start $)-6 . d 0 * t \_k(o p p(22)) *$ den

$\hookrightarrow \quad(\mathrm{x}, \mathrm{y}, \mathrm{start}) * \mathrm{u}_{-} 1 * \mathrm{zc}(\mathrm{opp}(22))$

$f(24, x, y$, start $)=f(o p p(24), x, y$, start $)-6 . d 0 * t \_k(o p p(24)) *$ den

$\hookrightarrow(\mathrm{x}, \mathrm{y}, \mathrm{start}) * \mathrm{u}_{-} 1 * \mathrm{zc}(\mathrm{opp}(24))$

$f(25, x, y$, start $)=f(o p p(25), x, y$, start $)-6 . d 0 * t \_k(o p p(25)) * d e n$

$\hookrightarrow(\mathrm{x}, \mathrm{y}, \mathrm{start}) * \mathrm{u}_{-} 1 * \mathrm{zc}(\mathrm{opp}(25))$

enddo

enddo

end if

$1 * * * * * * * * * * * * * * * * * * * * * * * * * * * * * * * * * * * * * * * * * * * * * * * * * * * * * * * * * * *$

$! ! * * * \$ O M P$ END DO

! BB at outlets

! ***********This Part applies the Adv-BC atthe OUTLETS of

$\hookrightarrow$ the T-Junction $* * * * * * *$

do $z=$ start, eend

if (z.gt. lz3p . and. z.lt.lz) then

do $y=2,1 y-1$ 


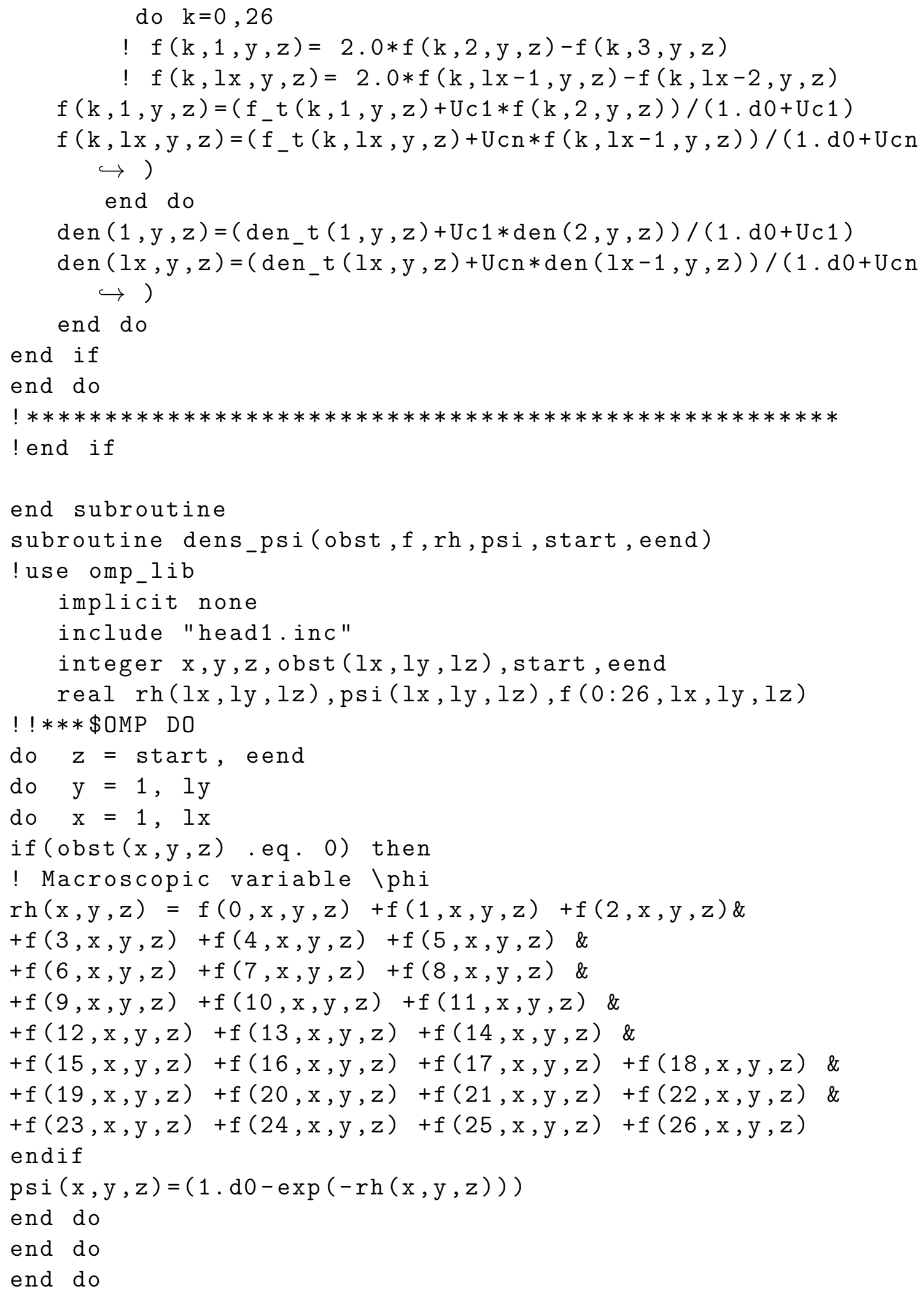




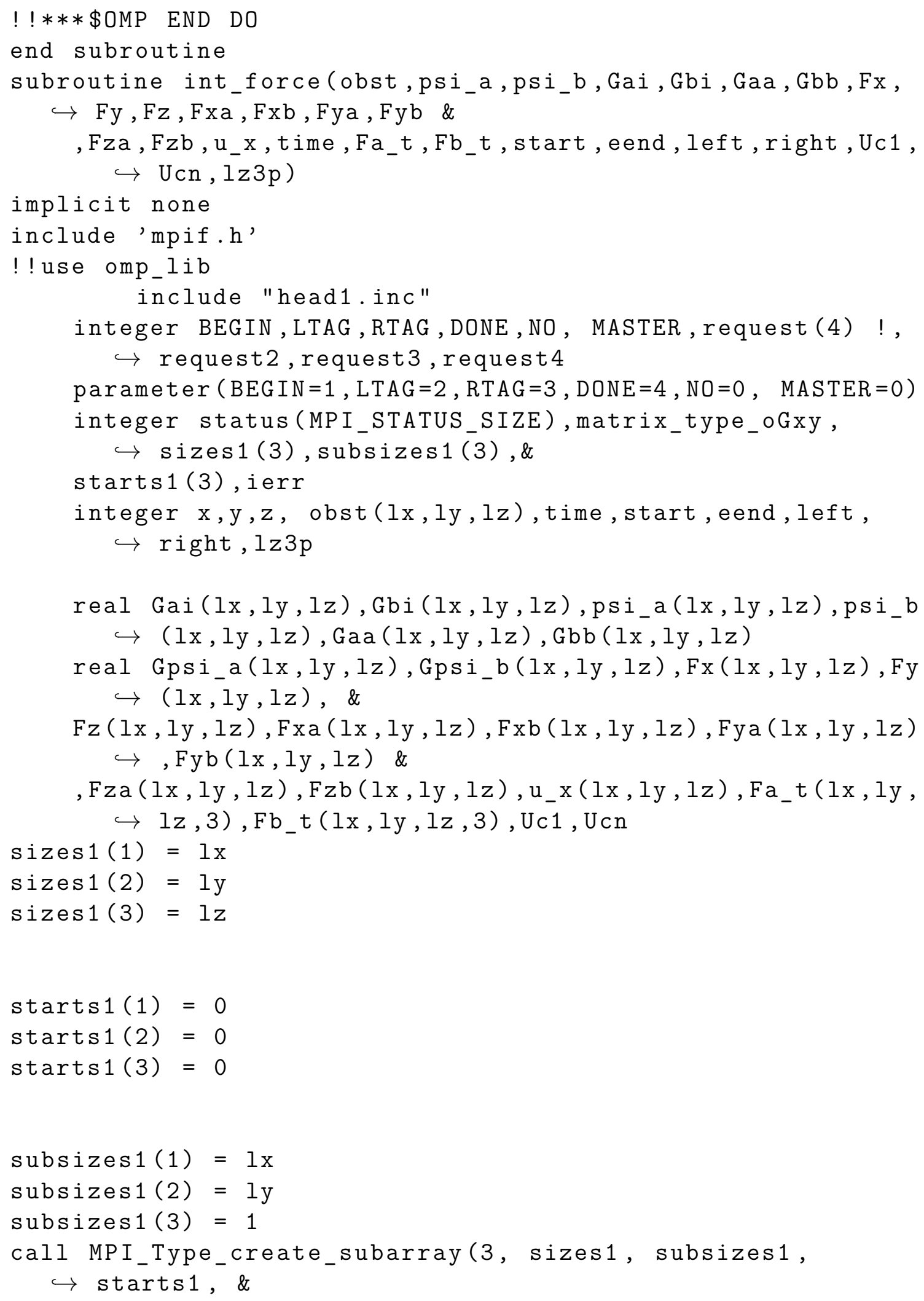




\begin{abstract}
MPI_ORDER_FORTRAN,
$\hookrightarrow$ MPI_REAL, \&

matrix_type_oGxy, ierr)

call MPI_Type_commit(matrix_type_oGxy, ierr)
\end{abstract}

! !***\$OMP workshare

Gpsi_a $(:$, , , start $:$ eend $)=G a i(:$, , start : eend $) *$ psi_b $(:$, , , start $\hookrightarrow$ : eend $)+$ \&

Gaa $(:$, :, start : eend $) *$ psi_a $(:$, , start : eend $)$

Gpsi_b (:, :, start : eend $)=G b i(:,:$, start : eend $) * p s i \_a(:,:$, start

$\hookrightarrow$ : eend $)+\&$

Gbb $(:$, :, start: eend $) *$ psi_b $(:,:$, start : eend $)$

!!***\$OMP END workshare

! $* * * * * * * * * * * * * *$ Non Blocking Send/Recv

$\hookrightarrow \quad * * * * * * * * * * * * * * * * * * * * * * * * * *$

call MPI_Sendrecv(Gpsi_a (1,1, start), 1, matrix_type_oGxy, $\hookrightarrow$ left,\&

RTAG,Gpsi_a $(1,1$, eend +1), 1, matrix_type_oGxy, right , \&

RTAG, MPI_COMM_WORLD, status, ierr)

call MPI_Sendrecv(Gpsi_a (1,1, eend), 1, matrix_type_oGxy, $\hookrightarrow$ right , \&

LTAG, Gpsi_a $(1,1$, start-1), 1, matrix_type_oGxy, left, \&

LTAG, MPI_COMM_WORLD, status, ierr)

call MPI_Sendrecv(Gpsi_b(1,1, start), 1, matrix_type_oGxy ,

$\hookrightarrow$ left,\&

RTAG,Gpsi_b $(1,1$, eend +1), 1, matrix_type_oGxy, right, \&

RTAG, MPI_COMM_WORLD, status, ierr)

call MPI_Sendrecv(Gpsi_b(1,1, eend), 1, matrix_type_oGxy,

$\hookrightarrow$ right , \&

LTAG, Gpsi_b $(1,1$, start-1), 1, matrix_type_oGxy, left, \&

LTAG, MPI_COMM_WORLD, status, ierr)

! call MPI_Barrier(MPI_COMM_WORLD, ierr)

! $* * * * * * * * * * * * * * * * * * * * * * * * * * * * * * * * * * * * * * * * * * * * * * * * *$

call FxFyFz(obst,psi_a,Gpsi_a, Fxa, Fya, Fza, u_x, Fa_t, start, $\hookrightarrow$ eend, left, right, Uc1, Ucn, lz3p)

call FxFyFz(obst,psi_b,Gpsi_b, Fxb,Fyb,Fzb, u_x, Fb_t, start, 
$\hookrightarrow$ eend, left, right, Uc1, Ucn, lz3p)

! !***\$OMP workshare

$$
\begin{aligned}
& F x(:,: \text {, start }: \text { eend })=F x a(:,: \text {, start }: \text { eend })+F x b(:,: \text {, } \\
& \hookrightarrow \text { start: eend) } \\
& \text { Fy }(:,: \text {, start }: \text { eend })=\text { Fya }(:,: \text {, start }: \text { eend })+\text { Fyb }(:,: \text {, } \\
& \hookrightarrow \text { start: eend) } \\
& \text { Fz }(:,: \text {, start }: \text { eend })=F z a(:,: \text {, start }: \text { eend })+F z b(:,: \text {, } \\
& \hookrightarrow \text { start: eend) }
\end{aligned}
$$

Fa_t $(:,:$, start $:$ eend, 1$)=F x a(:,:$, start $:$ eend $)$

Fa_t $(:,:$, start $:$ eend, 2$)=$ Fya $(:,:$, start $:$ eend $)$

Fa_t $(:,:$, start $:$ eend, 3$)=F z a(:,:$, start $:$ eend $)$

Fb_t $(:,:$, start $:$ eend, 1$)=F \times b(:,:$, start $:$ eend $)$

Fb_t $(:,:$, start $:$ eend, 2$)=$ Fyb $(:$, : , start $:$ eend $)$

Fb_t $(:,:$, start : eend , 3$)=F z b(:,:$, start $:$ eend $)$

! !***\$OMP END workshare

end subroutine

subroutine FxFyFz(obs,psi,Gpsi, F1,F2, F3, u_x, Ft, start, eend,

$\hookrightarrow$ left, right, Uc1, Ucn, lz3p)

implicit none

include 'mpif.h'

! ! use omp_lib

include "head1.inc"

integer obs $(l x, l y, l z), x, y, z, x p, y p, x n, y n, z p, z n$, start,

$\hookrightarrow$ eend, left, right, lz3p

real psi(lx,ly,lz), F1(lx,ly, lz), F2(lx,ly, lz), F3(lx,ly,

$\hookrightarrow 1 z), \operatorname{Gpsi}(1 x, 1 y, 1 z), \&$

$u_{-} x(1 x, 1 y, 1 z), F t(1 x, 1 y, l z, 3), U c 1, U c n$

$$
\begin{aligned}
& \text { !***\$OMP } \text { DO } \\
& \text { do } \mathrm{z}=\text { start }, \text { eend } \\
& \text { do } y=1, 1 \mathrm{y} \\
& \text { do } \mathrm{x}=1,1 \mathrm{x} \\
& \mathrm{xp}=\mathrm{x}+1 \\
& \mathrm{yp}=\mathrm{y}+1 \\
& \mathrm{zp}=\mathrm{z}+1 \\
& \mathrm{xn}=\mathrm{x}-1 \\
& \mathrm{yn}=\mathrm{y}-1 \\
& \mathrm{zn}=\mathrm{z}-1
\end{aligned}
$$

! Periodic boundary is applied here 


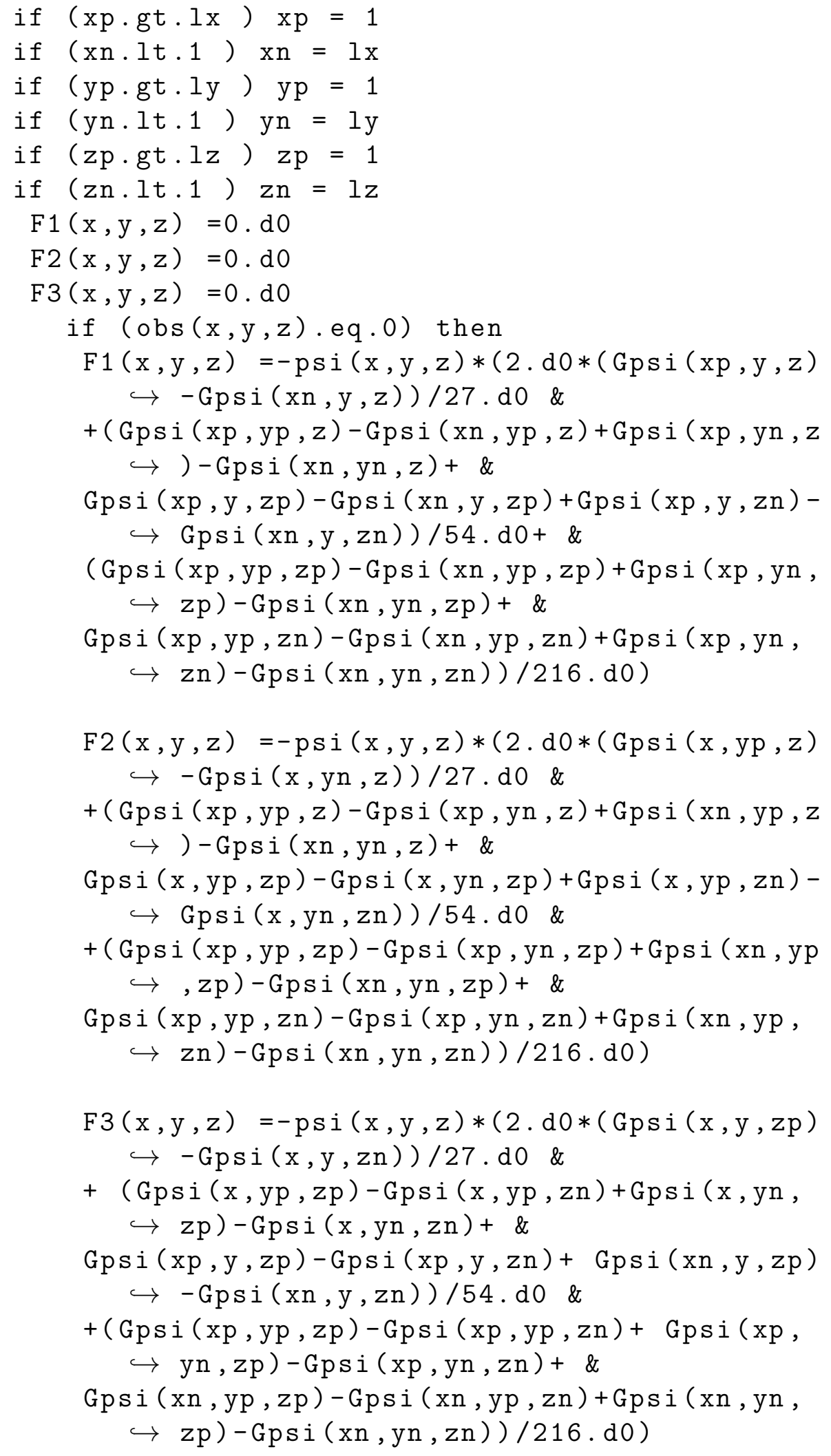




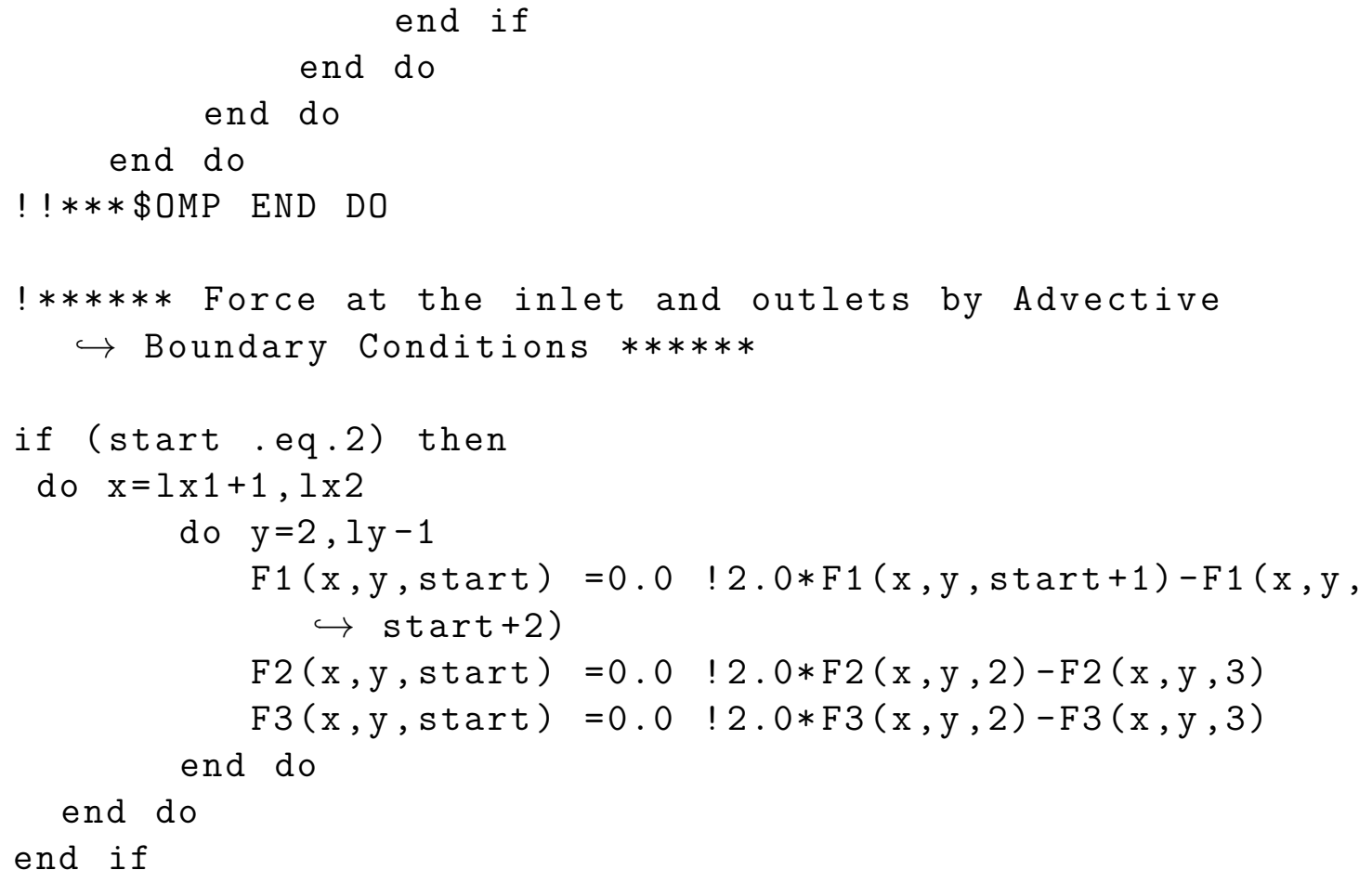




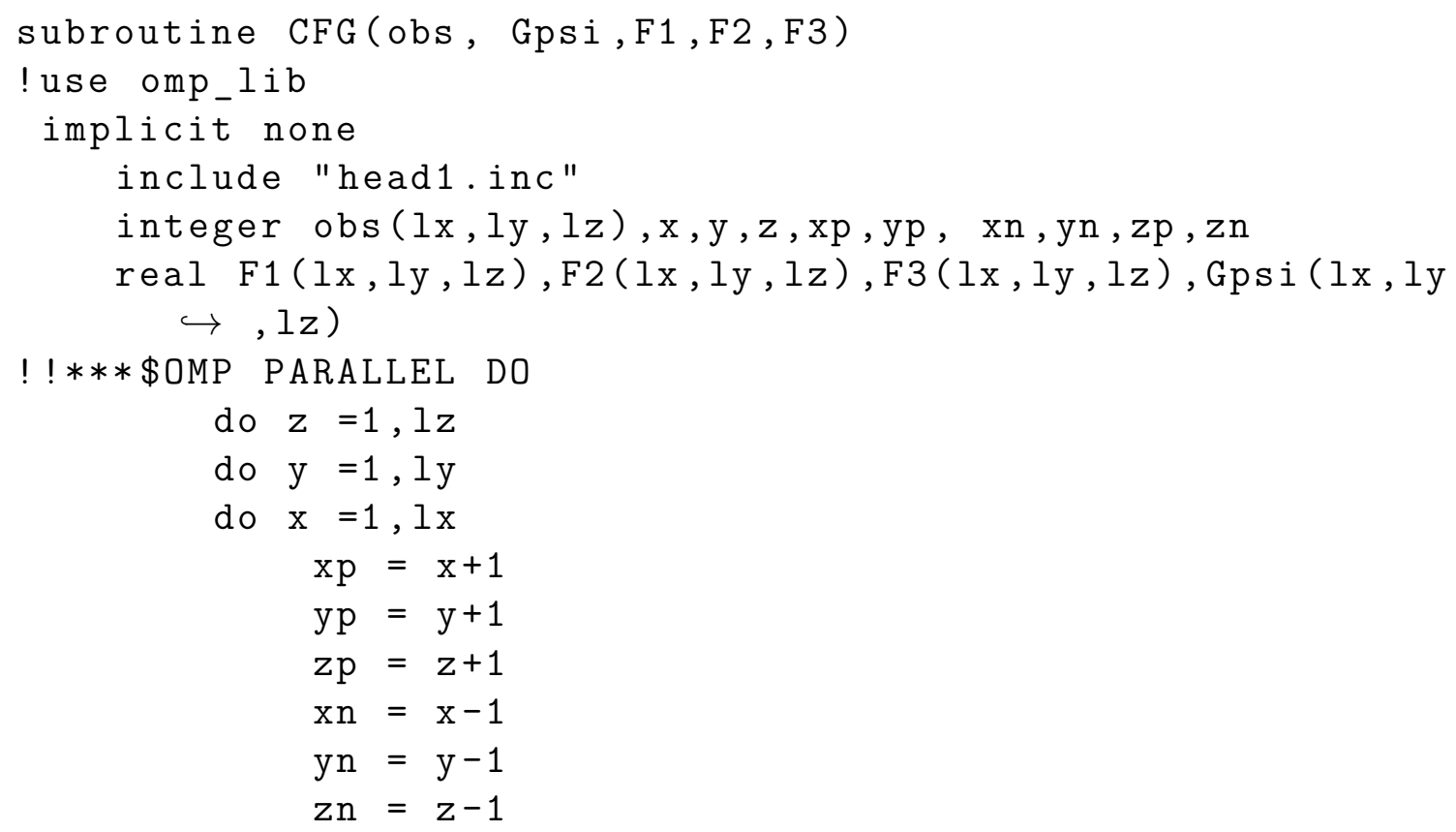

! Periodic boundary is applied here

$$
\begin{aligned}
& \text { if }(\mathrm{xp} \cdot \mathrm{gt} \cdot \mathrm{lx}) \mathrm{xp}=1 \\
& \text { if }(\mathrm{xn} .1 \mathrm{t} .1) \mathrm{xn}=1 \mathrm{x} \\
& \text { if (yp.gt.ly) yp }=1 \\
& \text { if (yn.lt.1) yn }=1 \mathrm{y} \\
& \text { if }(\mathrm{zp} \cdot \mathrm{gt} \cdot \mathrm{lz}) \mathrm{zp}=1 \\
& \text { if (zn.lt.1) } \mathrm{zn}=1 \mathrm{z} \\
& \mathrm{F} 1(\mathrm{x}, \mathrm{y}, \mathrm{z})=0 . \mathrm{d} 0 \\
& \mathrm{~F} 2(\mathrm{x}, \mathrm{y}, \mathrm{z})=0 . \mathrm{d} 0 \\
& \mathrm{~F} 3(\mathrm{x}, \mathrm{y}, \mathrm{z})=0 . \mathrm{d} 0 \\
& \text { if }(\text { obs }(x, y, z) . e q .0) \text { then } \\
& \mathrm{F} 1(\mathrm{x}, \mathrm{y}, \mathrm{z})=\operatorname{Gpsi}(\mathrm{xp}, \mathrm{y}, \mathrm{z})-\operatorname{Gpsi}(\mathrm{xn}, \mathrm{y}, \mathrm{z}) \text { \& } \\
& +\operatorname{Gpsi}(\operatorname{xp}, y p, z)-\operatorname{Gpsi}(x n, y p, z)+\operatorname{Gpsi}(x p, y n, z) \\
& \hookrightarrow-\operatorname{Gpsi}(\mathrm{xn}, \mathrm{yn}, \mathrm{z})+\& \\
& \text { Gpsi (xp,y, zp)-Gpsi (xn,y, zp) +Gpsi (xp,y, zn )- } \\
& \hookrightarrow \operatorname{Gpsi}(x n, y, z n)+\& \\
& \text { (Gpsi (xp,yp, zp)-Gpsi (xn,yp, zp) +Gpsi (xp,yn , } \\
& \hookrightarrow \mathrm{zp})-\mathrm{Gpsi}(\mathrm{xn}, \mathrm{yn}, \mathrm{zp})+\& \\
& \text { Gpsi (xp, yp, zn)-Gpsi (xn,yp, zn ) +Gpsi (xp, yn , } \\
& \hookrightarrow \mathrm{zn})-\mathrm{Gpsi}(\mathrm{xn}, \mathrm{yn}, \mathrm{zn})) \\
& \mathrm{F} 2(\mathrm{x}, \mathrm{y}, \mathrm{z})=(\operatorname{Gpsi}(\mathrm{x}, \mathrm{yp}, \mathrm{z})-\operatorname{Gpsi}(\mathrm{x}, \mathrm{yn}, \mathrm{z})) \& \\
& +(G p s i(x p, y p, z)-G p s i(x p, y n, z)+G p s i(x n, y p, z \\
& \hookrightarrow \quad)-\operatorname{Gpsi}(\mathrm{xn}, \mathrm{yn}, \mathrm{z})+\& \\
& \operatorname{Gpsi}(x, y p, z p)-\operatorname{Gpsi}(x, y n, z p)+\operatorname{Gpsi}(x, y p, z n)-
\end{aligned}
$$


$\hookrightarrow \operatorname{Gpsi}(\mathrm{x}, \mathrm{yn}, \mathrm{zn})) \&$

$+(\operatorname{Gpsi}(\mathrm{xp}, \mathrm{yp}, \mathrm{zp})-\mathrm{Gpsi}(\mathrm{xp}, \mathrm{yn}, \mathrm{zp})+\mathrm{Gpsi}(\mathrm{xn}, \mathrm{yp}$

$\hookrightarrow, \mathrm{zp})-\mathrm{Gpsi}(\mathrm{xn}, \mathrm{yn}, \mathrm{zp})+\&$

Gpsi (xp,yp, zn)-Gpsi (xp,yn, zn ) +Gpsi (xn, yp ,

$\hookrightarrow \mathrm{zn})-\mathrm{Gpsi}(\mathrm{xn}, \mathrm{yn}, \mathrm{zn}))$

$\mathrm{F} 3(\mathrm{x}, \mathrm{y}, \mathrm{z})=(\operatorname{Gpsi}(\mathrm{x}, \mathrm{y}, \mathrm{zp})-\operatorname{Gpsi}(\mathrm{x}, \mathrm{y}, \mathrm{zn})) \&$

$+(G p s i(x, y p, z p)-G p s i(x, y p, z n)+G p s i(x, y n, z p$

$\hookrightarrow)-\operatorname{Gpsi}(x, y n, z n)+\&$

$\operatorname{Gpsi}(x p, y, z p)-G p s i(x p, y, z n)+\operatorname{Gpsi}(x n, y, z p)$

$\hookrightarrow-\operatorname{Gpsi}(\mathrm{xn}, \mathrm{y}, \mathrm{zn}))$ \&

$+(\operatorname{Gpsi}(x p, y p, z p)-G p s i(x p, y p, z n)+G p s i(x p$,

$\hookrightarrow y n, z p)-G p s i(x p, y n, z n)+\&$

Gpsi (xn, yp, zp)-Gpsi (xn,yp, zn $)+G p s i(x n, y n$,

$\hookrightarrow \mathrm{zp})-\operatorname{Gpsi}(\mathrm{xn}, \mathrm{yn}, \mathrm{zn}))$

end if

end do

end do

end do

$! ! * * * \$ O M P$ END PARALLEL DO

! Force at the inlet and outlets by Extrapolation

! !***\$OMP PARALLEL DO

! do $\mathrm{x}=1 \times 1+1,1 \times 2$

! do $y=2,1 \mathrm{y}-1$

! $\quad \mathrm{F} 1(\mathrm{x}, \mathrm{y}, 1)=2.0 * \mathrm{~F} 1(\mathrm{x}, \mathrm{y}, 2)-\mathrm{F} 1(\mathrm{x}, \mathrm{y}, 3)$

! $\quad F 2(x, y, 1)=2.0 * F 2(x, y, 2)-F 2(x, y, 3)$

! $\quad \mathrm{F} 3(\mathrm{x}, \mathrm{y}, 1)=2.0 * \mathrm{~F} 3(\mathrm{x}, \mathrm{y}, 2)-\mathrm{F} 3(\mathrm{x}, \mathrm{y}, 3)$

! end do

! end do

!!***\$OMP END PARALLEL DO

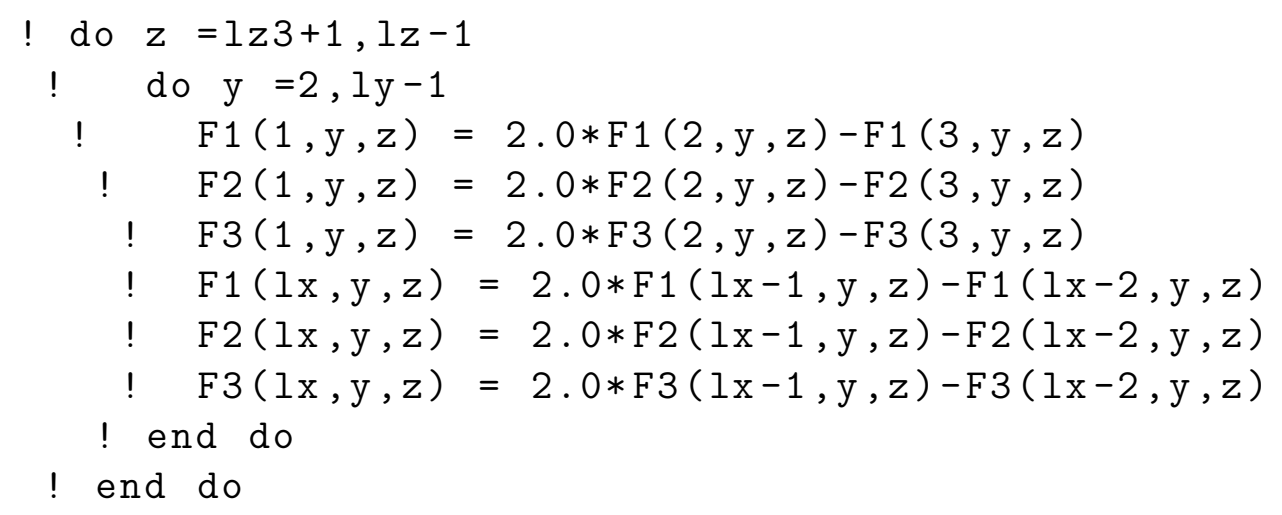




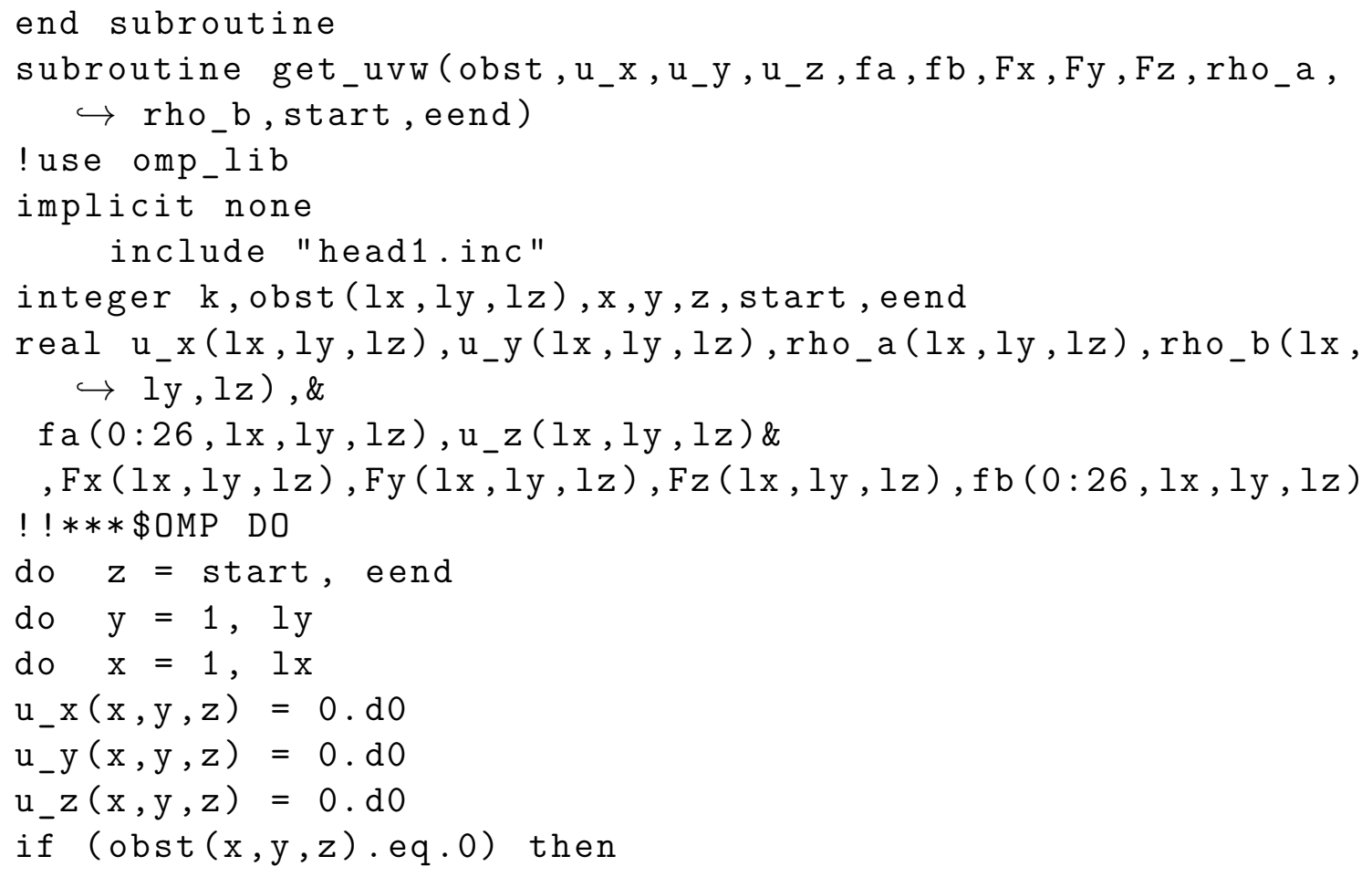




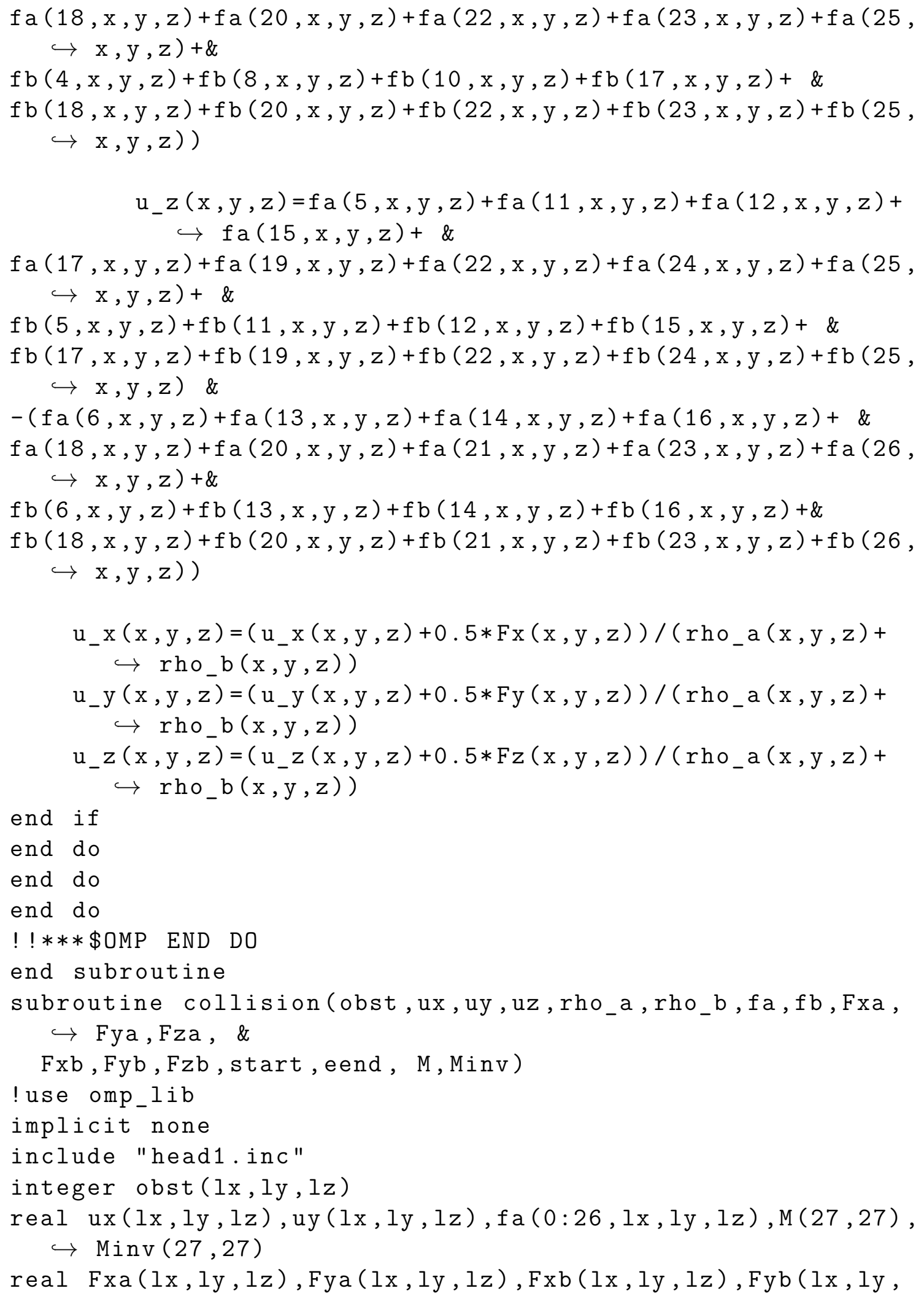


$\hookrightarrow 1 \mathrm{z})$

real Fza(lx, ly, lz), Fzb(lx,ly, lz), uz(lx, ly, lz), fb(0:26, lx, $\hookrightarrow 1 \mathrm{y}, 1 \mathrm{z}), \quad \&$

rho_a $(l x, l y, l z), r h o \_b(l x, l y, l z), u n c, p h i$, taum

integer $x, y, z, k$, start, eend

real feq_a $(0: 26)$, feq_b $(0: 26), F_{-} a(0: 26), F_{-} b(0: 26)$

! !***\$OMP DO

do $z$ start, eend

do $y=1,1 \mathrm{y}$

do $x=1,1 x$

if (obst $(x, y, z)$.eq. 0) then

call f_eq (rho_a $(x, y, z), u x(x, y, z), u y(x, y, z), u z(x, y, z)$,

$\hookrightarrow$ feq_a)

call f_eq ( rho_b $(x, y, z), u x(x, y, z), u y(x, y, z), u z(x, y, z)$,

$\hookrightarrow$ feq_b)

!****** Component $\mathrm{xz}$ of Deviatoric Stress******

do $\mathrm{k}=0,26$

unc $=x c(k) * u x(x, y, z)+y c(k) * u y(x, y, z)+z c(k) * u z(x, y, z)$

$\mathrm{F}_{-} \mathrm{a}(\mathrm{k})=\mathrm{t}_{-} \mathrm{k}(\mathrm{k}) *((3.0 *(\mathrm{xc}(\mathrm{k})-\mathrm{ux}(\mathrm{x}, \mathrm{y}, \mathrm{z}))+9.0 * \mathrm{unc} * \mathrm{xc}(\mathrm{k})) *$

$\hookrightarrow \quad \mathrm{Fxa}(\mathrm{x}, \mathrm{y}, \mathrm{z}) \&$

$+(3.0 *(\mathrm{yc}(\mathrm{k})-\mathrm{uy}(\mathrm{x}, \mathrm{y}, \mathrm{z}))+9.0 * \mathrm{unc} * \mathrm{yc}(\mathrm{k})) * \mathrm{Fya}(\mathrm{x}, \mathrm{y}, \mathrm{z})+\&$

$(3.0 *(\mathrm{zc}(\mathrm{k})-\mathrm{uz}(\mathrm{x}, \mathrm{y}, \mathrm{z}))+9.0 * \mathrm{unc} * \mathrm{zc}(\mathrm{k})) * \mathrm{Fza}(\mathrm{x}, \mathrm{y}, \mathrm{z}))$

$\mathrm{F}_{-} \mathrm{b}(\mathrm{k})=\mathrm{t}_{-} \mathrm{k}(\mathrm{k}) *((3.0 *(\mathrm{xc}(\mathrm{k})-\mathrm{ux}(\mathrm{x}, \mathrm{y}, \mathrm{z}))+9.0 * \mathrm{unc} * \mathrm{xc}(\mathrm{k}))$

$\hookrightarrow * \mathrm{Fxb}(\mathrm{x}, \mathrm{y}, \mathrm{z}) \&$

$+(3.0 *(\mathrm{yc}(\mathrm{k})-\mathrm{uy}(\mathrm{x}, \mathrm{y}, \mathrm{z}))+9.0 * \mathrm{unc} * \mathrm{yc}(\mathrm{k})) * \mathrm{Fyb}(\mathrm{x}, \mathrm{y}, \mathrm{z})+\&$ $(3.0 *(\mathrm{zc}(\mathrm{k})-\mathrm{uz}(\mathrm{x}, \mathrm{y}, \mathrm{z}))+9.0 * \mathrm{unc} * \mathrm{zc}(\mathrm{k})) * \mathrm{Fzb}(\mathrm{x}, \mathrm{y}, \mathrm{z}))$

end do

$p h i=\left(r h o_{-} a(x, y, z)-r h o_{-} b(x, y, z)\right) /\left(r h o_{-} a(x, y, z)+r h o_{-} b(x, y\right.$, $\hookrightarrow \mathrm{z})$ )

taum $=(1 \cdot d 0+p h i) * t a u_{-} a / 2 \cdot d 0+(1 \cdot d 0-p h i) * t a u_{-} b / 2 \cdot d 0$

$f a(:, x, y, z)=f a(:, x, y, z)-\left(f a(:, x, y, z)-f e q \_a(:)\right) /$ taum

$\hookrightarrow+(1.0-0.5 /$ taum $) * F_{-} a(:)$

$f b(:, x, y, z)=f b(:, x, y, z)-\left(f b(:, x, y, z)-f e q \_b(:)\right) /$ taum

$\hookrightarrow+(1.0-0.5 /$ taum $) * F_{-} b(:)$

! call mrt_collision ( $f a(0: 26, x, y, z)$, feq_a, taum, F_a, M, Minv)

! call mrt_collision ( $f b(0: 26, x, y, z)$, feq_b, taum, F_b, M, Minv)

end if

end do

end do

end do

! !***\$OMP END DO

end subroutine 


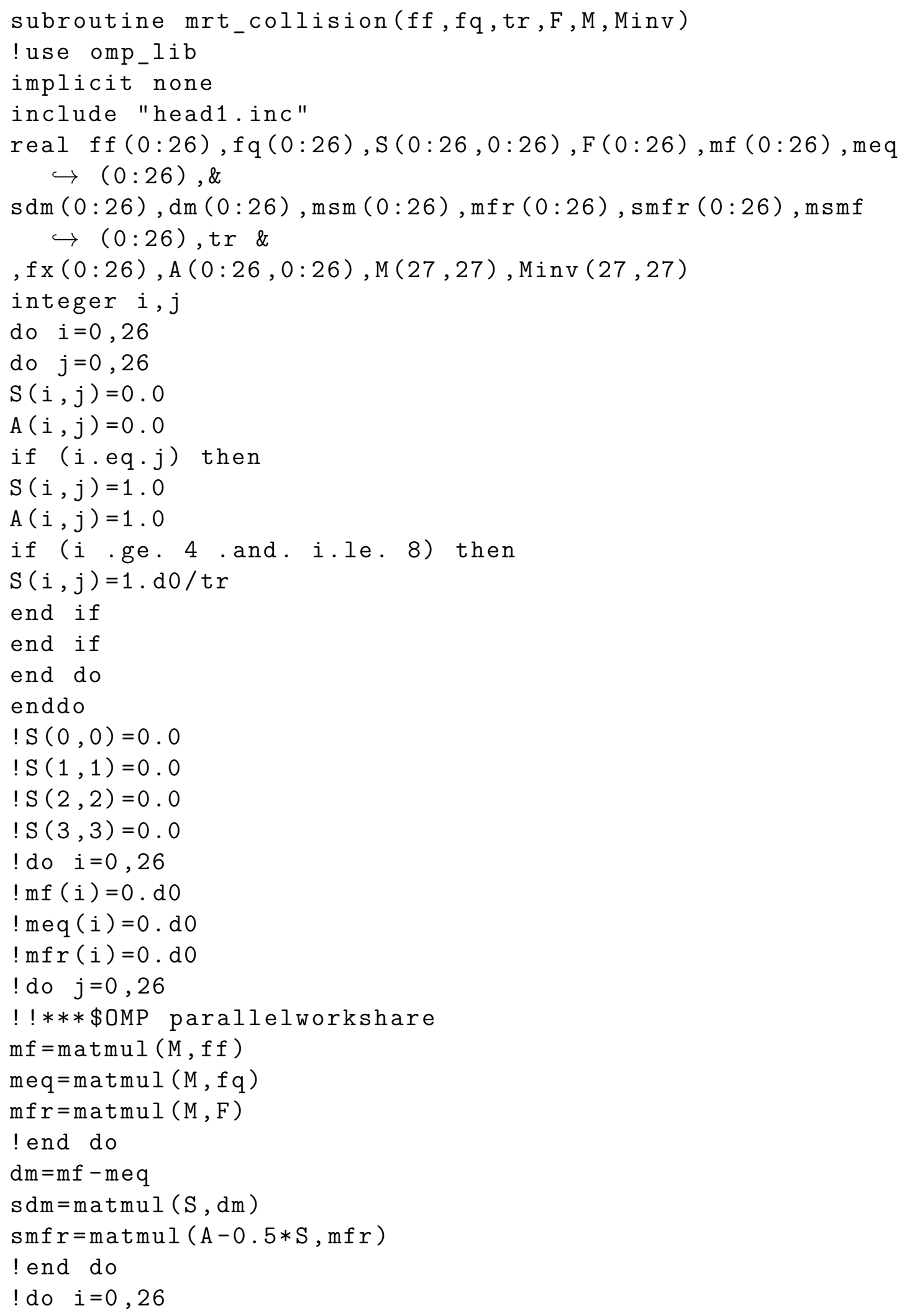




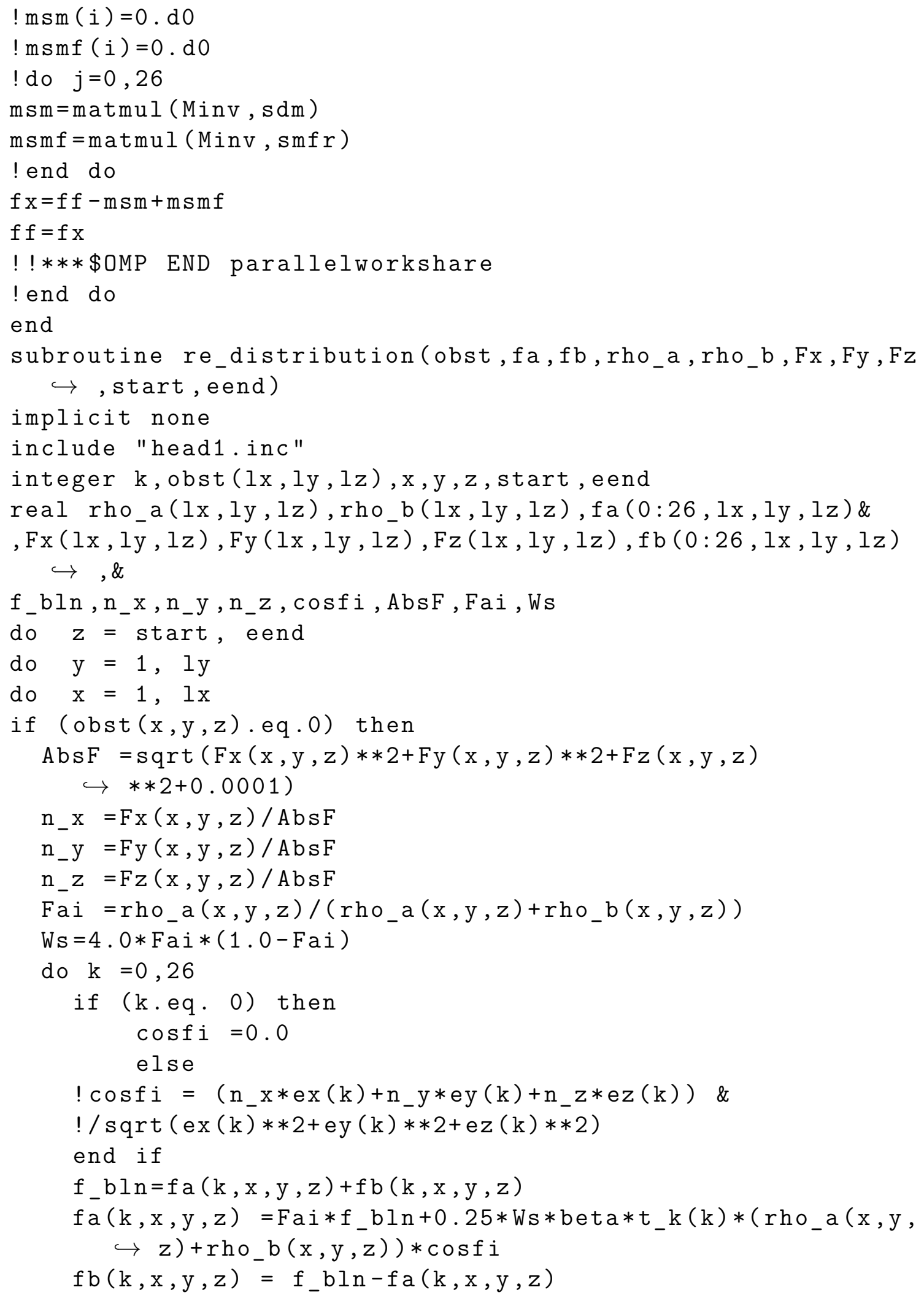




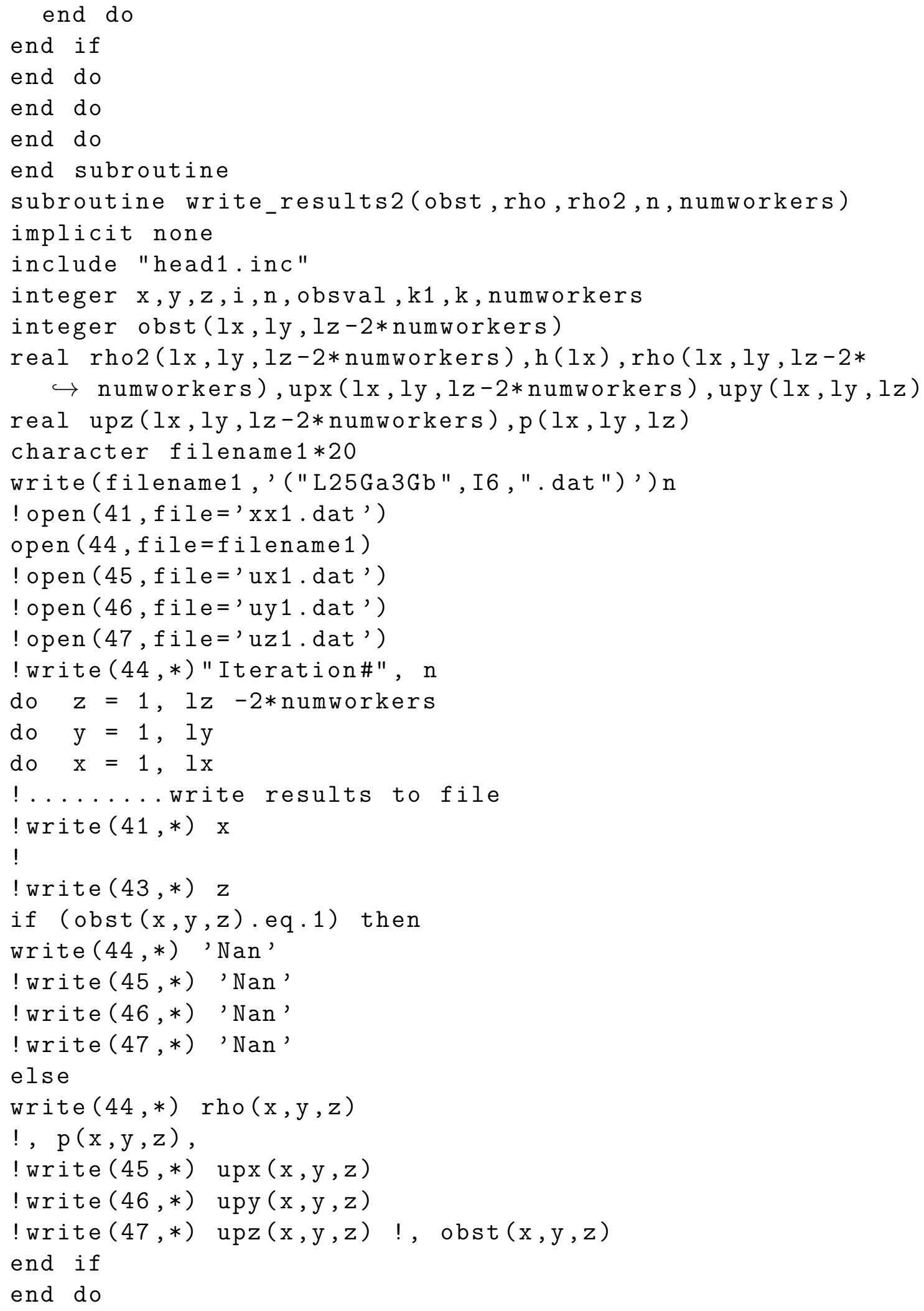




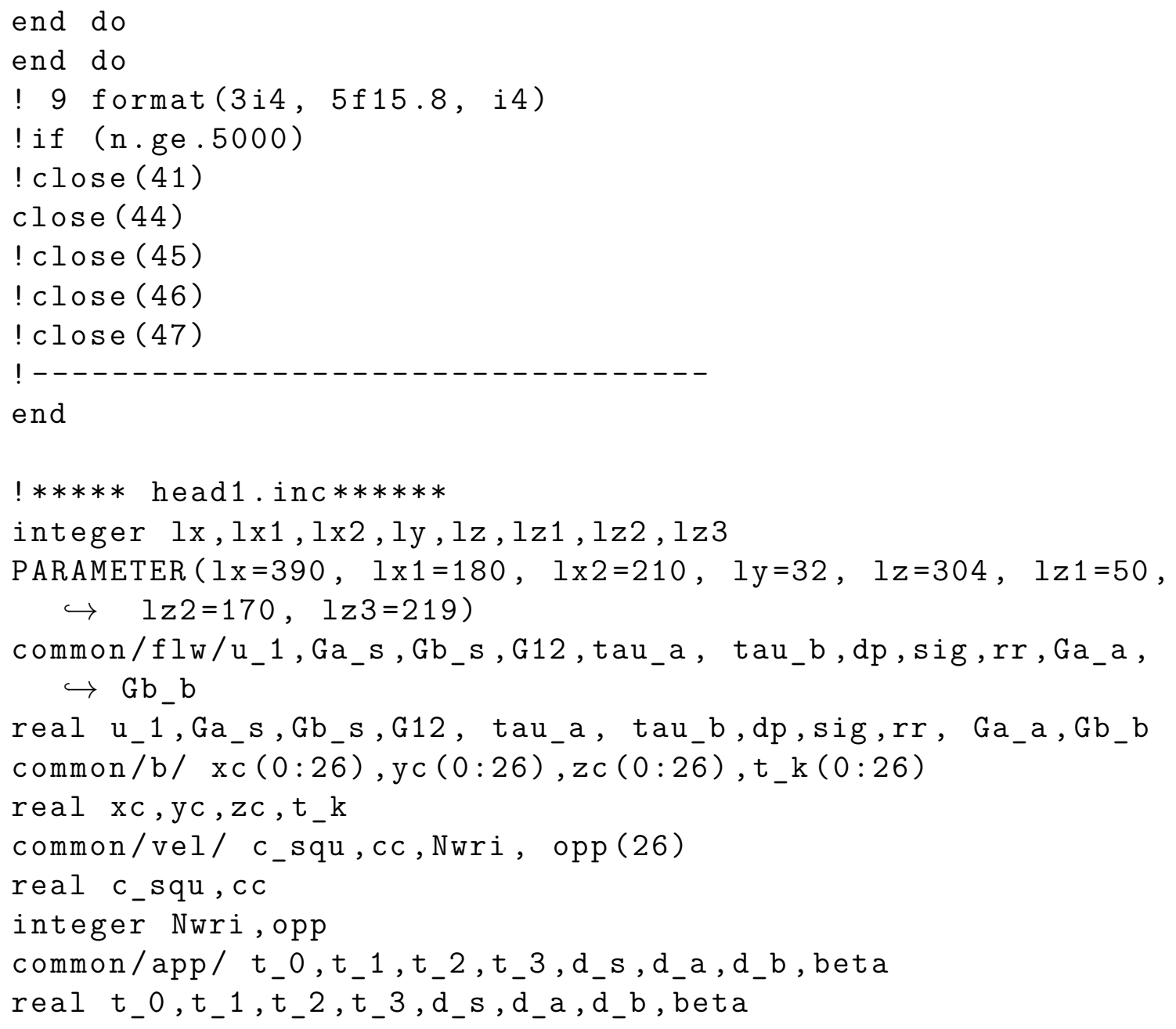

